In This Issue:

Proceedings of the

Symposium on Animal

Welfare and Scientific

Research: 1985 to 2010 


\section{ILAR Council}

\section{ILAR Journal Editorial Board}

Myrtle Davis, Editor in Chief

Nelson L. Garnett

Daniel S. Marsman

Melinda A. Novak

\section{Issue Editor}

Susan B. Silk

\section{Managing Editor}

Cameron H. Fletcher

\section{ILAR Staff}

Frances E. Sharples, Acting Director Lida Anestidou, Senior Program Officer Cameron H. Fletcher, Senior Editor Jason Worthy, Senior Program Assistant
Floyd E. Bloom, Chairman

Molecular and Integrative Neuroscience

Department

Scripps Research Institute

La Jolla, CA

Kathryn A. Bayne

Association for Assessment

and Accreditation of Laboratory

Animal Care International

Frederick, MD

Myrtle Davis

National Cancer Institute

National Institutes of Health

Bethesda, MD

Nelson L. Garnett

Consultant, Laboratory Animal Care and

Use Programs

Vass, NC

Judy A. MacArthur Clark

Animals Scientific Procedures Inspectorate

London, UK

Daniel S. Marsman

Personal Health/Feminine Care Safety

Procter \& Gamble

Mason, $\mathrm{OH}$

Garry Neil

Corporate Office of Science and Technology

Johnson \& Johnson

New Brunswick, NJ
Timo Olavi Nevalainen

National Laboratory Animal Center

University of Eastern Finland

Kuopio, Finland

Steven M. Niemi

Center for Comparative Medicine

Massachusetts General Hospital

Charlestown, MA

Melinda A. Novak

Department of Psychology

University of Massachusetts

Amherst, MA

Menelas Pangalos

Innovative Medicine

AstraZeneca

Alderley Park, UK

Bernard E. Rollin

Colorado State University

Fort Collins, CO

James A. Roth

College of Veterinary Medicine

lowa State University

Ames, IA

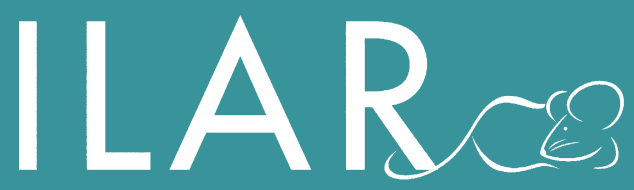

INSTITUTE FOR LABORATORY RESEARCH
ILAR Journal is the quarterly, peer-reviewed publication of the Institute for Laboratory Animal Research (ILAR), The National Academies, 500 Fifth Street, NW, Washington, DC 20001; Tel: 202334-2590; Fax: 202-334-1687; E-mail: ILARJ@ nas.edu; URL: www.nationalacademies.org/ilar. Membership in the ILAR Associates Program includes four issues of ILAR Journal per year. Subscribe: Online at www.nationalacademies.org/ilar, or contact ILAR for more information. Online Access: Articles are available online 6 months after publication, free of charge. Single Copy Sales: ILAR Journal issues can be purchased online in hard copy. Reprints: To order reprints, go to the ILAR Journal homepage, download the Reprint Order Form, and follow submission instructions. Permissions: Permission to use or reprint text or figures is granted for educational or research purposes only. Please ensure the high quality of the reproduction and include a credit line to identify the article as an original publication in ILAR Journal. The credit line should appear as follows: "Reprinted with permission from ILAR Journal, (include Volume and Issue Number), Institute for Laboratory Animal Research, The National Academies, 500 Fifth Street NW, Washington DC 20001 (www.nationalacademies.org/ilar)." Indexing: ILAR Journal is indexed by AGRICOLA, EMBASE, MEDLINE, and Thomson Scientific (in Current Contents $₫ /$ Agriculture, Biology, and Environmental Sciences; Current Contents®/Life Sciences; Focus On: Veterinary Science \& Medicine; and Science Citation Index Expanded [also known as SciSearch $®]$ ).

ILAR Journal ISSN 1084-2020

Volume 52, Supplement, 2011

Copyright 2011 by the Institute for Laboratory Animal Research. All rights reserved. Printed in the United States of America on acid-free paper. 


\section{INSTITUTE FOR LABORATORY ANIMAL RESEARCH}

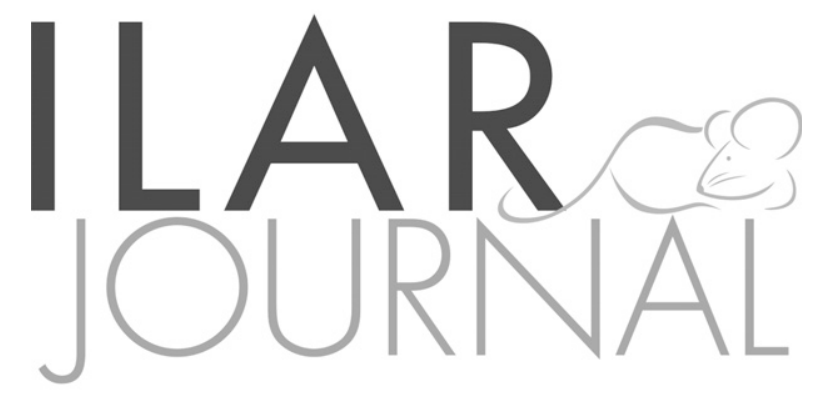

NATIONAL RESEARCH COUNCIL

OF THE NATIONAL ACADEMIES

2011

Volume 52, Supplemental issue

Proceedings of the Symposium on Animal Welfare and Scientific Research: 1985 to 2010

417 Symposium Welcome

Patricia Brown and Chester Gibson

419 Introduction: By Ladies and Gentlemen, for Ladies and Gentlemen Nelson Garnett

422 Animal Housing Facilities: Session Introduction Taylor Bennett

423 Where We Came From

Betty Goldentyer and Christian Newcomer

427 How We Got There

Lauretta Gerrity

428 Animal Facility Improvement Project Funding Willie McCullough

430 Where We Are Today Steven Leary

432 Animal Housing Facilities: Discussion

434 Institutional Animal Care and Use Committees: Session Introduction James Taylor

434 Formative Years

Ralph Dell

436 The IACUC's Impact on Advancing Best Practices and the Three Rs Kathryn Bayne 
439 The Future: Continuing IACUC Challenges Jerry Collins

442 Institutional Animal Care and Use Committees: Discussion

445 Education and Training: Session Introduction Mary Lou James

446 Conmed: The First Educational Forum Steele Mattingly

448 The Role of Education in Self-Evaluation Susan Silk

450 Education and Training: Discussion

451 Veterinary Care: Session Introduction Patricia Brown

452 Veterinary Care: Where We Were Gerald Van Hoosier

453 Current Standard of Care and Technologies: Nonrodents Michael Talcott

458 Current Standard of Care and Technologies: Rodents William White

463 Improving Animal Housing and Welfare Brian Hare

468 Veterinary Care: Discussion

469 Synergy of Working Together Dale Schwindaman and John Miller

473 Synergy of Working Together: Discussion

474 Keynote Address: Standing on the Shoulders of Giants Charles McCarthy

479 Reflections on IRAC and the US Government Principles Robert Whitney and Thomas Wolfle

482 Perspectives of PHS Funding Agencies: Session Introduction Susan Silk

482 Use of Animals in NIH-Supported Biomedical Research Sally Rockey

484 Global Impact of Animal Research on Infectious Diseases: A CDC Perspective Tanja Popovic 
488 Use of Animals in the Development of Medicines and Devices for Humans and Animals

David Jacobson-Kram

491 Perspectives of PHS Funding Agencies: Discussion

493 Animal Models and Basic Science-Bench to Bedside: Session Introduction

Richard Nakamura

493 Animal Models and Basic Science-Bench to Bedside Richard Nakamura

498 Animal Models in Immunology and Transplant Medicine Linda Cendales

501 Animal Models Facilitate Rapid Responses to Emerging Infectious Diseases

Michael Kurilla

506 Animal Models and Basic Science-Bench to Bedside: Discussion

509 Insights into the Brain and Aging: Session Introduction Stuart Zola

509 Challenges of an Aging Brain

Stuart Zola

514 Genetic Vulnerabilities Associated with Cancer and Aging Nancy Hopkins

520 Toward a Better Understanding of Depression, Schizophrenia, and Autism Larry Young

524 Insight into Brain Development from Animal Vocalization Studies Erich Jarvis

531 Insights into the Brain and Aging: Discussion

534 Standing Up for Science: Session Introduction Jerry Collins

534 Ways Individual Scientists and Clinicians Can Support Animal Research J. David Jentsch

537 The Need for Public Dialogue Dario Ringach

539 Standing Up for Science: Discussion

541 Program: Symposium on Animal Welfare and Scientific Research: 1985 to 2010 
545 List of Speakers

547 Symposium Welcome

548 NIH Record of the 1984 Symposium

550 Timeline of the History of Animal Welfare Policy, Regulation, and Guidance

552 US Government Principles for the Utilization and Care of Vertebrate Animals Used in Testing, Research, and Training

553 List of Abbreviations

554 Thanks to Institutional Supporters

555 ILAR Journal Subscription Order Form

557 ILAR Publications

561 ILAR Publications Order Form

563 ILAR Journal Individual Issues Order Form 


\section{About ILAR}

he Institute for Laboratory Animal Research (ILAR) promotes high-quality, humane care of animals and the appropriate use of animals and alternatives. As a component of the Division on Earth and Life Studies, ILAR functions within the mission of the National Academy of Sciences as an independent adviser to the federal government, the biomedical research community, and the public.

ILAR was founded in 1952 under the auspices of the National Research Council, National Academy of Sciences. The National Research Council serves as an independent adviser to the federal government on scientific and technical questions of national importance. Jointly administered by the National Academy of Sciences and the National Academy of Engineering, the National Research Council brings the resources of the entire scientific and technical community to bear on national problems through its volunteer advisory committees.

ILAR Journal is the quarterly, peer-reviewed, theme-oriented publication of ILAR, which provides timely information for all those who use, care for, and oversee the use of animals in research. According to policy, any previously unpublished animal research reported in ILAR Journal will have been conducted according to the scientific, technical, and humanely appropriate guidelines current at the time the research was conducted, according to the Guide for the Care and Use of Laboratory Animals. Opinions expressed in this journal are not necessarily those of ILAR, the National Research Council, or the National Academy of Sciences.

Publication of this special supplemental issue of the ILAR Journal was supported by funds from the Association for Assessment and Accreditation of Laboratory Animal Care (AAALAC) International, the American Association for Laboratory Animal Science (AALAS), IACUC 101, the National Institutes of Health (NIH) Office of Laboratory Animal Welfare (OLAW) and National Institute of Mental Health (NIMH), Public Responsibility in Medicine and Research (PRIM\&R), and the Scientists Center for Animal Welfare (SCAW).

\section{Support ILAR's Mission by Becoming an ILAR Associate}

- All ILAR Associates receive the quarterly ILAR Journal at more than $50 \%$ off the cover price. ILAR Journal is the only peer-reviewed journal dealing with issues of specific interest to both IACUCs and research scientists.

- All ILAR Associates receive a discount on National Academies Press publications.

- Institutional and Supporting Associates have online access to current ILAR Journal issues.

\begin{tabular}{|l|c|c|}
\hline Associate Type* & Annual Rate US (\$) & Annual Rate Non-US (\$) \\
\hline Student & 60 & 80 \\
\hline Individual & 100 & 120 \\
\hline Institutional & 850 & 950 \\
\hline Supporting & 1,675 & 1,775 \\
\hline $\begin{array}{l}\text { *Institutional Associates receive 10 subscriptions and copies of the Guide upon } \\
\text { request. Supporting Associates receive 20 subscriptions and copies of the Guide, and 1 } \\
\text { copy of current ILAR publications upon request. Student Associates must provide } \\
\text { mentor's signature (post-doctoral status is not included). }\end{array}$ \\
\hline
\end{tabular}

Use ILAR's secure site to join, order, or renew: www.nationalacademies.org/ilar 


\title{
In the Next Issue
}

\section{Neurobiology of Addictive Behavior}

\author{
Volume 52, Number 4
}

Scientific Editors: Kimberlei A. Richardson and Gabrielle L. McLemore

\section{Planned contents:}

- The Neurobiology of Addiction-like Behaviors - Elisabeth Van Bockstaele

- Contributions of Animal Models to Clinical Treatment of Addictions-Po Hsiung Peter Wu and Kalynn M. Schulz

- Addiction-Related Gene Regulation: Cognitive Enhancers vs. Cocaine and Amphetamine - Vincent Van Waes and Heinz Steiner

- Influence of Sex Differences and Gonadal Hormones on Cocaine Addiction-Shirzad Jenab and Vanya Quiñones-Jenab

- Rodent Models of Binge Eating: Are They Models of Addiction? - Rebecca L. Corwin and R. Keith Babbs

- Neurobiology of Consummatory Behavior: Mechanisms Underlying Overeating and Drug Use - Jessica Barson, Irene Morganstern, and Sarah F. Leibowitz

- Translational Neuroimaging in Drug Addiction and Obesity-Michael Michaelides, Panayotis K. Thanos, Nora D. Volkow, and Gene-Jack Wang

- IACUC Considerations Specific to the Use of Animal Models in Studies on the Neurobiology of Addictive Behaviors - Doris E. Hughes Moore

Become an ILAR Associate and save!

\begin{tabular}{|l|c|c|}
\hline Associate Type* & Annual Rate US (\$) & Annual Rate Non-US (\$) \\
\hline Student & 60 & 80 \\
\hline Individual & 100 & 120 \\
\hline Institutional & 1,675 & 950 \\
\hline Supporting & 1,775 \\
\hline $\begin{array}{l}\text { * Student Associates must provide mentor's signature (postdoctoral status is not } \\
\text { included). Institutional Associates receive 10 subscriptions, online access, and copies } \\
\text { of the Guide upon request. Supporting Associates receive 20 subscriptions, online } \\
\text { access, and copies of the Guide and 1 copy of current ILAR publications upon request. }\end{array}$ \\
\hline
\end{tabular}

Subscribe or order online:

www.ilarjournal.com 


\title{
Animal Welfare and Scientific Research: 1985 to 2010 October 25, 2010
}

\author{
Symposium Welcome
}

Patricia Brown

'd like to welcome you all today. I'm Director of the Office of Laboratory Animal Welfare $\left(\mathrm{OLAW}^{1}\right)$ at the National Institutes of Health (NIH), and I welcome you to what I hope will be a very interesting and unique symposium honoring the past and looking forward to the future of laboratory animal welfare and science. This symposium would not be possible without the support of our other two sponsors, the United States Department of Agriculture (USDA) Animal and Plant Health Inspection Service (APHIS) Animal Care and IACUC 101.

We also have a number of other supporters. Our federal partners are the Centers for Disease Control and Prevention (CDC), the Department of Veterans Affairs (VA), and the Food and Drug Administration (FDA), as well as the following NIH institutes and centers: the Eunice Kennedy Shriver National Institute of Child Health and Human Development (NICHD), the National Cancer Institute (NCI), the National Eye Institute (NEI), the National Heart, Lung, and Blood Institute (NHLBI), the National Human Genome Research Institute (NHGRI), the National Institute on Aging (NIA), the National Institute on Alcohol Abuse and Alcoholism (NIAAA), the National Institute of Allergy and Infectious Diseases (NIAID), the National Institute of Arthritis, Musculoskeletal, and Skin Diseases (NIAMS), the National Institute on Drug Abuse (NIDA), the National Institute of Mental Health (NIMH), the National Institute of Environmental Health Sciences (NIEHS), and the Office of Animal Care and Use (OACU) Intramural Research Program, NIH Office of the Director.

We had additional supporters that included the following organizations: the Association for Assessment and Accreditation of Laboratory Animal Care (AAALAC) International, the American Veterinary Medical Association (AVMA), the

\footnotetext{
The transcripts included in this special issue are those that were reviewed by the speakers, who also gave written permission for their publication. Also included are the Symposium program; List of speakers, Welcome statement; NIH Record of the 1984 Symposium; Timeline of the History of Animal Welfare Policy, Regulation, and Guidance; and US Government Principles for the Utilization and Care of Vertebrate Animals Used in Testing, Research, and Training, reproduced on pages 541-552.

${ }^{1}$ For a list of abbreviations used in these transcripts, see page 553.
}

Collaborative Institutional Training Initiative (CITI), Charles River Laboratories, the National Association for Biomedical Research (NABR), and Public Responsibility in Medicine and Research (PRIM\&R).

Twenty-five years ago, significant events occurred in the United States that advanced the welfare of research animals. Today we want to not only reflect on those advances but also discuss future directions for the oversight and care of laboratory animals. Many of our resources for the housing and care of animals, and for gathering data from research with animals, have vastly changed. We will be exploring those changes and we will hear about cutting-edge technologies and equipment that could not be forecast in 1985. These resources now aid in minimizing the use of animals and often refine or eliminate distress and pain. The oversight of biomedical research using animals has been transformed through the requirement for institutional animal care and use committees (IACUCs), the evolution of monitoring, and the focused commitment to appropriate animal care and use at the local level.

Some of our future leaders are sitting here in the room today. I'd like to especially welcome the Next Generation Travel Award recipients. These veterinary students and laboratory animal medicine residents come from across the United States to learn from us and to better understand animal welfare and science. They are Gillian Braden-Weiss, Michael Mahmoud Esmail, Dr. James Finlay, Diane Guerrero, Dr. Anna Hampton, Ian Hannigan, Dr. Jennifer Lofgren, Dr. Nicole Monts de Oca, and Dr. Quynh Tien Ngoc Tran. Special thanks are extended to the NIEHS and IACUC 101 because, along with OLAW, they supported the Next Generation Travel Awards. We hope that the awardees are inspired by our sharing of the past and our discussion of where the past should lead.

The schedule for today will include a series of four panel discussions followed by question and answer sessions. The two panel topics in the morning are Animal Housing Facilities and Animal Care and Use Committees; the afternoon panels will focus on Education and Training and Veterinary Care. This will be followed by a presentation on the Synergy of Working Together by Dr. John Miller, formerly of the NIH Office for 
Protection from Research Risks (OPRR), the predecessor to OLAW, and Dr. Dale Schwindaman, formerly of USDA APHIS Animal Care. Immediately following the program, there will be a dinner and keynote address. We'd like to thank AAALAC International for their support of those activities. Dr. Charles McCarthy, the former OPRR director, will provide the keynote address; and he will be joined by Dr. Robert Whitney and Dr. Thomas Wolfle, who will share their reflections on the development of the US Government
Principles and the early work of the Interagency Research Animal Committee (IRAC).

This symposium was reviewed and approved by the American Association of Veterinary State Boards (AAVSB) Registry of Approved Continuing Education (RACE) program, for continuing education credits in jurisdictions that recognize AAVSB approval.

I now turn to Dr. Chester Gipson, Deputy Administrator, APHIS Animal Care, for his welcome from USDA.

\section{Chester Gipson}

$\mathrm{t}$ is my pleasure to welcome you to the Animal Welfare and Scientific Research Symposium, honoring the past and looking forward to the future direction of laboratory animal welfare. While honoring the past, we should not forget to honor those who were on the front line leading the initiative, such as two of our distinguished speakers just mentioned, Dr. Dale Schwindaman and Dr. John Miller. Also, I would be remiss if I did not mention the late Christine Stevens of the Animal Welfare Institute, who was a very strong advocate of animal welfare.

The significant event that occurred in the United States 25 years ago, advancing the welfare of animals, marked the end of what I would refer to as the animal welfare revolution and started what I refer to as the animal welfare evolution. It is animal welfare evolution that we will discuss today as we explore the advances made in animal welfare and research. These advances remind me of two quotes. The first is by William James: "the greatest discovery of my generation is that man can alter his life by altering his attitude of mind." And that's part of what happened 25 years ago. The second quote is by Tommy Lasorda: "The difference between the impossible and the possible lies in a person's determination." And that is what this evolution is about-individual determination to continue to move welfare and research forward into the future. It is through shared determination and ultimately attitude that we will continue the progress of the last 25 years, thus catapulting us into the future.

It is now my pleasure to introduce Dr. Nelson Garnett. $\mathrm{He}$ is a 1972 graduate of the University of Georgia School of Veterinary Medicine and a Diplomate of the American College of Laboratory Animal Medicine. After fulfilling his obligation in the US Air Force, Dr. Garnett practiced small animal medicine in Charlottesville, Virginia. He then completed a residency in laboratory animal medicine at the Johns Hopkins University and went on to direct the Laboratory Animal Program at the University of Maryland School of Medicine. Since 1987 Dr. Garnett has held various positions at NIH and OPRR and for approximately 3 years served as the director of OLAW. In 2004, after 20 years of combined federal service, he retired from the US Public Health Service as director of OLAW. Dr. Garnett was a corecipient of the 2005 Scientists Center for Animal Welfare (SCAW) Harry Rowsell Award and in 2006 was appointed to the Institute for Laboratory Animal Research (ILAR) Council and the Board of Directors of Americans for Medical Progress (AMP). He currently consults and serves as a member of the Johns Hopkins Animal Care and Use Committee. 


\section{Introduction: By Ladies and Gentlemen, for Ladies and Gentlemen}

\section{Nelson Garnett}

$\mathrm{t}$ is my honor and pleasure to be here with you this morning. I thank the organizers of this meeting for the invitation to address this audience on a subject that is near and dear to my heart.

I've been asked to give a very brief introduction to this 25-year Policy anniversary celebration by giving a bit of information and historical context, leading up to the pivotal 1985 time period when so many important events affecting biomedical research were occurring. But first, a word about the title of my talk.

Early in my NIH career, while attending an OPRRsponsored educational workshop, I was listening to a university administrator from the host institution give his welcoming remarks. In those remarks, he described the Public Health Service Policy as having been written "by ladies and gentlemen, for ladies and gentlemen." And then-with apologies to our very good friends at USDA - he went on to describe the Animal Welfare Act (AWA) as having been written for "dog thieves and smugglers."

A light bulb went off in my head at that moment because he really captured the essence of many of the different statutory mandates, the agency missions, and the organizational cultures behind the evolving animal welfare mechanisms in the United States. This dichotomy could also be used to describe some of the fundamental differences between regulation versus policy, minimum or design standard versus performance standard, and monitored self-regulation versus an inspection-based regulation system.

The laws, regulations, and policies in the United States come from three main sources; two are government and one is a private voluntary accreditation body. They are the USDA, the Department of Health and Human Services (DHHS), and AAALAC International. I will focus mostly on the government contribution, although there are many similarities among the three.

I prefer to think of all three of these bodies as part of a system of oversight. It may seem strange, but this is not a system of contradictory, competing rules. Instead, one of the greatest strengths of the US system lies in the complementary nature of these three organizations. Each has its own separate jurisdiction, which frequently overlaps with the others, and each has its own specialty niche that contributes to the effectiveness of the whole system. And as you will learn later today, it is no accident that these three organizations work together as harmoniously as they do. Most universities develop institutional policies that apply uniformly to all of the animal activities, choosing the most stringent of the three requirements in order to ensure consistency and compliance.

I would now like to very briefly point out some littleknown information about the evolution of animal welfare policies on the NIH Public Health Service side. This particular policy was reportedly posted on a sign in the Hygienic Laboratory, the precursor of NIH, in 1904: "Animals are to be used in the proper work of the lab, but anything which inflicts pain upon them will not under any circumstances be allowed."

In 1912, decisions regarding animals were made at the highest levels. Then the National Institute (and it was singular at that time) of Health was established in 1930.

Here are just a few other NIH historical milestones. The NIH campus in Bethesda was established in 1937. In 1950, the NIH director issued the first Rules Regarding Animals. These were subsequently revised as part of the official NIH Manual. In 1966, as alluded to earlier, the original Animal Welfare Act dealt primarily with pet theft, with many amendments being added, up to the major changes in 1985. It was also during this period that the Animal Care Panel was morphing into the present-day organizations of the American Association for Laboratory Animal Science (AALAS) and AAALAC. The precursor to the Guide for the Care and Use of Laboratory Animals was first published.

In 1971 we saw the first application of animal care policies to institutions receiving NIH awards. In 1973, these policies were expanded to cover all of the Department of Health, Education, and Welfare (DHEW), which is now the DHHS. The possibility of sanctions was introduced into these policy revisions.

Next came the formation of OPRR (now OLAW), with responsibility for human subjects' protection and animal welfare oversight, with corresponding revisions of the Guide and the PHS Animal Welfare Policy. Remember that during much of the 1970s, institutions receiving PHS funding could demonstrate compliance with NIH policies by simply being AAALAC accredited.

Bob Whitney and Tom Wolfle will cover these subjects in detail, but I want to call your attention to the first memorandum of understanding (MOU) among the NIH, USDA, and FDA. This helped provide the essential groundwork for future interagency negotiation and harmonization of animal welfare regulation and policy. Table 1 summarizes these events. 


\section{Table 1 Timeline of events that preceded the 1985 Public Health Service Policy and Amendment to the Animal Welfare Act}

\begin{tabular}{|c|c|}
\hline 1904 & $\begin{array}{l}\text { "Animals are to be used in the proper work of the lab, but anything which inflicts pain upon them will not under any } \\
\text { circumstances be allowed." - Dr. Milton Rosenau, Director, Hygienic Laboratory }\end{array}$ \\
\hline 1912 & Hygienic Laboratory renamed the Public Health Service (PHS) \\
\hline 1930 & Ransdell Act established the National Institute of Health (NIH) \\
\hline 1935 & Bethesda site purchased for $\$ 10$ \\
\hline 1935 & Franklin Delano Roosevelt signed Social Security Act, authorizing $\$ 2$ million to the states for investigating disease \\
\hline 1937 & $\mathrm{NIH}$ relocated to Bethesda \\
\hline 1950 & NIH director issued "Rules Regarding Animals" \\
\hline 1954 & "Rules Regarding Animals" revised as NIH Manual \\
\hline 1966 & Public Law 89-544, the Animal Welfare Act \\
\hline 1971 & $\begin{array}{l}\text { NIH "Policy, Care, and Treatment of Laboratory Animals": First NIH "policy" applicable to institutions receiving or } \\
\text { about to receive NIH grant or contract awards }\end{array}$ \\
\hline 1973 & $\begin{array}{l}\text { Revision of NIH Policy: } \\
\text { - Expanded coverage DHEW-wide } \\
\text { - Sanctions possible } \\
\text { - "DHEW Principles" }\end{array}$ \\
\hline 1974 & Institutional Relations Branch becomes Office for Protection from Research Risks (OPRR) \\
\hline 1978 & Revised Guide \\
\hline 1979 & Revised PHS "Animal Welfare Policy" \\
\hline 1982 & Trans-NIH Coordinating Committee \\
\hline 1983 & Interagency Research Animal Committee (IRAC) \\
\hline 1983 & $\begin{array}{l}\text { Memorandum of Understanding (MOU) among NIH, USDA, FDA: Groundwork for future negotiation and } \\
\text { harmonization }\end{array}$ \\
\hline 1985 & Guide revision \\
\hline
\end{tabular}

During this time other events were happening, too. In the early 1980s we had the Silver Spring monkey episode that launched the animal rights organization PETA (People for the Ethical Treatment of Animals). In the mid-80s we saw the University of Pennsylvania baboon head trauma exposé and the lengthy investigations that followed. This was a time of intense animal rights activism, which undoubtedly influenced public opinion and Congress during the critical period leading up to 1985 .

Now we come to that red-letter year, 1985. The 1985 Guide revision was issued and is referenced in the existing PHS Policy as the standard upon which institutions must base their programs of animal care and use as a condition of eligibility for funding. Also note that the Guide not only addressed animal welfare-related issues but also had a major impact on producing high-quality reproducible research through the elimination of unwanted variables.

In November of 1985, Congress passed Public Law 99158, the Health Research Extension Act of 1985, which authorized the existing PHS Policy almost verbatim. OPRR had already started establishing institutional Assurances with all awardee institutions under the existing Policy and these required only slight modification to conform to the changes that were made in the Public Law itself. The Health Research Extension Act of 1985 amended the Public Health Service Act to include the Public Health Service Policy on Humane Care and Use of Laboratory Animals (PHS Policy), implemented by OPRR.
It is interesting to note that the PHS Policy preceded the legislation that authorized it. So it was already being implemented immediately before and after passage. It is widely believed that Congress was familiar with the contents of the PHS Policy and provided a strong endorsement by authorizing it virtually unchanged. It's also interesting to note that the Health Research Extension Act was passed about one month prior to the 1985 Food Security Act, which included the major amendments to the Animal Welfare Act. This was interpreted as meaning that Congress intended the Animal Welfare Act Regulations to be compatible with the recently passed Health Research Extension Act and PHS Policy. The applicability of the PHS Policy includes all animal-related activities conducted or supported by the PHS. This coverage is very broad. It includes intramural and extramural research, grants and contracts, subcontracts, training grants, cooperative agreements, domestic and foreign activities, and even some collaborations and things like purchase orders.

OPRR was named as being responsible for implementing the PHS Policy. It occupied a very unusual position in the chain of command of NIH and DHHS. The Act itself explicitly tasks the Secretary of DHHS, acting through the Director of NIH, with responsibility for implementation of the PHS Policy, PHS-wide. OPRR also reported to the Director of NIH, through the Deputy Director of Extramural Research. Thus we had a small office deep within the NIH Office of the Director with oversight that included NIH intramural research as well as the intramural research 
programs of parallel agencies of CDC and FDA. This placement did on occasion result in some tensions when it was necessary for OPRR to assert its PHS authority at higher levels in the chain of command.

In addition to the PHS agencies, many others-the National Science Foundation, Howard Hughes Medical Institute (HHMI), American Heart Association-have adopted the PHS Policy for their own grant funding programs. This has greatly expanded the influence of the Policy beyond its original jurisdiction.

The PHS Policy places direct responsibility on the NIH and other PHS funding components for ensuring that these requirements have been met before making awards. The language - "no award shall be made without an Assurance and without IACUC approval"-makes it clear that part of the burden is on the funding components themselves. Again we had the potential within the NIH hierarchy. OPRR's administrative location in the Office of Extramural Research had oversight responsibility for its own bosses' compliance with the PHS Policy requirements, in addition to its bosses' boss. However, I'm pleased to report that OPRR, and later OLAW, at least during my tenure, was always supported on those occasions when it had to assert authority across, over, or up the chain of command.

This is just to emphasize the tight relationship between PHS animal welfare policy and PHS grants policy. Each grant applicant must address five points in the application as part of the scientific peer review process. This is in contrast to the AWA regulatory framework. It is important to note that compliance with the PHS Policy is essentially one of the terms and conditions of award, not purely regulation; I'm sure our USDA speakers will cover this subject in much more detail later today. I just want to reemphasize that the date of passage for the Animal Welfare Act Amendment, December 1985, was approximately 1 month after the Health Research Extension Act was passed.

Also note that in 1985 the USDA implementing regulations had not been written. That process was to take up to 6 years to complete and early drafts of the Animal Welfare Act Regulations made it clear that major interagency effort was required if the resulting regulations were to be compatible with the already well developed and fully implemented PHS Policy requirements. We will hear much more about that aspect of interagency harmonization later in the day. However, I wish to point out the importance of the groundwork that had been laid for the work that was to come.

In closing, I want to reemphasize the special significance of the MOUs of interagency cooperation that I mentioned earlier. These were subsequently renewed. I also want to point out some very explicit provisions in AWA itself. Specifically, it requires the Secretary of Agriculture to consult with the Secretary of Health and Human Services prior to issuance of regulations. As you will hear later, this played a role in the extraordinary negotiations that took place during the harmonization of two distinctly different sets of rules and two distinctly different regulatory cultures. 


\section{Animal Housing Facilities: Session Introduction}

\section{Taylor Bennett}

t's a pleasure to moderate this session on animal housing facilities. When I first got involved in the care of laboratory animals (I think it was 1950), our facility was called the Animal House. It was located next to the Residents' Housing on the VA Station where I lived until I was 8 years old; I was taking care of guinea pigs on weekends for about 50 cents. Twenty-eight years later, I became the director of the Biological Resources Laboratory (BRL) at the University of Illinois at Chicago (UIC). I hope none of you postdocs out there take that long to achieve success. The BRL, often called the animal hospital by many of the users, was in fact a state-of-the-art facility for the time, having been the initial facility where the Illinois Cubicles were developed and used. By the time I retired from the UIC, the BRL had undergone many major and minor renovations to become what it is-and what most other animal housing facilities are today-an extension of the investigator's laboratory.

In putting this session together, my goal was to call attention to the progress that has been made in managing the environment in which laboratory animals are maintained. There are many reasons for this improvement but, based on my experience, the primary driver has been to minimize the impact of the housing environment on the data generated using animal-based models. The principal goal of an institutional animal care and use program should be to optimize the well-being of animals that are cared for and used in that program. Optimizing animal welfare will ensure compliance with the regulations and standards of the USDA, the PHS Policy, and the accreditation requirements of AAALAC International. Optimizing animal welfare will also minimize nonexperimental variability and thus facilitate the research that must use animals.

Today's speakers have been chosen to highlight how far we have come in terms of the physical environment in which we house animals; to discuss how we got there by improving the facilities in which the animals are maintained and the equipment that is used to support that maintenance; and finally to look at trends in facility design and construction.
I will keep the introductions brief so our speakers can concentrate on what you came to hear.

Dr. Betty Goldentyer is the Eastern Regional Director for the APHIS Animal Care program at USDA. Betty has been with the Animal Care program since its organization as a standalone program in 1988. She will present the USDA's perspective on how far we've come in terms of facility issues.

Dr. Chris Newcomer is the Executive Director of AAALAC International and has participated in its review activities for the past 25-plus years. He will present AAALAC's perspective on how far we have come.

Dr. Lauretta Gerrity is the Associate Vice President for Research Operations and Compliance and a professor in the Department of Genetics at the University of Alabama in Birmingham (UAB). She was previously Director of the Animal Research Program there. I was lucky enough to have some exposure to that program and that's why I asked Lauretta to speak. She is going to talk about the commitment that institutions must make when they seek to obtain funding for facility improvements.

Dr. Willie McCullough has been a Health Science Administrator at NCRR since 2001. He served as Director of the Animal Facilities Improvement Program and the Research Facilities Improvement Program from January 2001 to March 2010. He will talk about NCRR's tremendous commitment to the process of improving animal housing facilities.

And Dr. Steve Leary is the Assistant Vice Chancellor for Veterinary Affairs and Director of the Division of Comparative Medicine at Washington University School of Medicine in St. Louis. He has over 30 years of experience in laboratory animal medicine and management of laboratory animal facilities. He has coauthored chapters on animal facility design and the use of interstitial mechanical space in animal facility design in the most recent edition of Laboratory Animal Medicine in the ACLAM (American College of Laboratory Animal Medicine) book series. He will address trends in facility design. 


\title{
Where We Came From
}

\author{
Betty Goldentyer
}

want to give you some idea what it was like to be a young USDA inspector in the late 1980s and early '90s. It's just my perspective and I am going to generalize a little from my own experience.

I started out with USDA in 1988 in North Dakota and did animal welfare inspections there and then I moved to Wisconsin and was responsible for Wisconsin, a little of Minnesota, and I dipped down into Chicago. In 1991 I moved to Florida and was the regional animal care specialist, so I got to ride with inspectors in facilities all throughout the southeast. That was exactly when the implementing regulations were coming into effect and we were trying to figure out what the heck we were going to do with them. It was also the time, not coincidentally, that USDA was moving from animal welfare as a collateral duty for veterinarians and animal health technicians whose primary duty was animal health.

We were building up a program that's a whole inspection force dedicated to animal welfare. That was a big change for the Department at the time-setting up a whole new program and working with new regulations. There were major fundamental changes in how an inspector does the job and how you look at the animals that you see every day. The changes were as basic as, What do we consider to be humane care? In the past we were looking at cage sizes, veterinary care, food and water. Now we're thinking about environmental enrichment and exercise, about what the animal's life is really like.

Bringing on programmatic oversight was a big changethe fact that now we were not the only ones looking at the programs, we were looking at another program, which is looking at the animals. That was completely new to us. And another big change was the idea of minimum standards versus performance standards and thinking about best practices. Before we figured out how to deal with all of that, the USDA inspector rolled into the facility about once a year, and there were no other bodies conducting inspections. So from my point of view, when I would get to a facility, it was something new and exciting every day.

Of course, I am generalizing, but things were a lot less centralized than they are now. We would go to a university, I would go to a facility, and a principal investigator (PI) in psychology would take you around the psychology department, and then they would hand you off to the farm manager and they would tell you how they do business. Then they would take you back to the physiology department and you'd meet someone else and they'd show you their program. It really was challenging to even find all the animals and figure out what was going on.
At one small college, I was going around the facility with a facility representative. She didn't know where all the animals were, I didn't know where all the animals were. We went into a laboratory and there's the PI's bench. We pulled the drawer open - and there was a box of mice. Now, that's not a regulated species and those mice were absolutely fine, but it does give you an idea of how far we've come in terms of institutional responsibility and oversight.

Another thing that really struck me, looking back on those early inspections, was what a vacuum we lived in then. I lived just outside Madison and on Monday morning I would get in my old Plymouth K car-I had a stack of brown file folders and a map of Wisconsin — and I would just say "See you Friday." No cell phone, no computer-we didn't have the kind of communication, the networking, and the resources that we do now. We pretty much were inventing it as we went along.

In the facilities and housing areas we had new regulations. Rabbit cage size changes came in 1990 and right after that we had primate cage size changes. Then we had environmental enrichment and dog exercise regulations, which completely changed the way we looked at animal housing. We had the Pet Protection Act, which changed the way we looked at record keeping and certification. And all of that without even getting into the major IACUC changes. Think about what it was like for the inspector, bringing all those changes on, but you were out there all by yourself. It is not a good day for an inspector when you have to break it to the facility that the cage sizes are changing. You don't want to be the one that brings that news to them.

I'm going to tell a true story on Taylor [Bennett] and myself. I was invited to a branch AALAS meeting outside Madison, I think it was 1989 or ' 90 . I got to the meeting and Taylor had a photocopy of the proposed implementing regulations that I had not seen. I said, "Oh, can I have a copy of your regulations? Which are really our regulations." That gives you an idea that we were working hard to cooperate and communicate, but without the tools we have now. The resources we now have at hand are great. Getting information upfront lets you make good decisions before you find some paperwork you didn't know was even there.

I know I am not the only one in the room who remembers this, but back in the day we used to have long philosophical discussions about rust and how much rust is too much. We actually trained our inspectors in evaluating rust to look at the pitting, the flaking. "Is it sanitizable?" "Is it structurally sound?" It's amazing to think about the amount of time we 
spent on that and it's great not to have to deal with that kind of minimum-standard approach. I completely credit performance standards for taking us away from those rust discussions.

When we brought in performance standards, I don't think anybody knew how it was going to work for a USDA inspector. I certainly didn't. We didn't know how we were going to work with facilities using the moving targets that are performance standards. "How are we going to do this?" "In the event that we need an enforcement case, how are we going to bring that forward?" We really did not know how to do that. We've taken our time and it hasn't been a completely straight path but I think we worked it out. The greatest gift of those performance standards has been moving away from that minimalistic approach so that we can all look at what's best for the animal and not be thinking about what meets those minimum requirements.

Another thing that rarely comes up any more when you're out there at a facility is questioning about the basic need for the Animal Welfare Act. "Why do we even have this federal regulation? Why is the government in my laboratory?" We used to hear this. And being asked "Why are you here? What value do you add?" That's perfectly fine-I expect all of our inspectors to be able to articulate what it is that we are there to do and why it's important. But how we're going to make this process better is the discussion we want to be having. And we don't hear that basic questioning any more like we used to. We moved from "Why are you here? Why do we have an Animal Welfare Act?" to "How can we keep making this better and better?"

There is a lot of wisdom in the IACUC system and the way the Regulations and the Animal Welfare Act were changed, with the clear responsibility on the institution to protect the resource, to provide the best animal welfare and the best science possible. The overall emphasis on training and qualifications makes it so that everyone throughout the facility understands what their job is and takes great care of the animals. Identifying a whole committee dedicated to animal welfare highlights the commitment to the animals. You put all of that together and it creates a culture of animal welfare, and for a USDA inspector that's exactly where you want to be working every day. I am proud to have worked with you all as you have created that.

\section{Christian Newcomer}

t's great to be with you today to share my personal experiences and relate recent information from AAALAC International about its findings in the review of facilities now versus those of previous years and to discuss some of the influences that played a role in that transition.

My career includes about 25 years of involvement with AAALAC. I first began working in laboratory animal medicine under the 1978 Guide for the Care and Use of Laboratory Animals as a postdoctoral scholar in the University of Michigan Training Program in 1978. It's very interesting to note the language of that Guide, because it sets the stage for understanding the scope of the transition that we were able to achieve over the subsequent 30 years in our improvements in animal care and use programs.

If you look at the 1978 Guide's approach, you note very scant mention of the concept of "institution." For example, in the introduction, one of the few references to the institution states, "The operation of the institutional animal facilities should be in accord with the recommendations presented in this Guide." Later it states, "It is hoped that this Guide will encourage investigators to seek new and better methods of laboratory animal care." This statement places the emphasis on the investigators and not the institution, so it's reaching out to investigators. This reflects the fact that animal care and use programs in many institutions were very much laboratory- and department-based in 1978. The idea that we would [work] to improve care at the institutional level is not something that the institution was asked to consider. Progress in care and use initiatives had to be taken to individual investigators to encourage them to look for new and improved methods in the quality of animal care and use. However, there also was a short chapter on institutional policies in the
1978 Guide that did advise on the utility of a committee on animal care and use as a way to help your program improve.

The 1985 Amendments to the Animal Welfare Act and PHS Policy on Humane Care and Use of Laboratory Animals really did usher in a new era. The language in the latter document immediately evokes the concept of the institutional program on animal care and use, and later states, "the Assurance shall fully describe the institution's program for the care and use of animals in PHS-conducted or -supported activities." The PHS Policy requires institutions to use the Guide for the Care and Use of Laboratory Animals as a basis for implementing an institutional program for activities involving animals. This is a very different focus from that reflected in the 1978 Guide.

The 1985 Guide is identified as the regulatory document for organizations that receive PHS funding. The PHS emphasis on institutional responsibility buttressed by the 1985 Guide yielded this clear reorientation in the Preface: "The purpose of this Guide is to assist institutions [emphasis added] in caring for, and using, laboratory animals in ways judged to be professionally and humanely acceptable." So the PHS Policy and Guide no longer appealed primarily to investigators to adopt new methods that may improve laboratory animal care. Instead, these documents mandated and instructed institutions to conduct programs that are professionally and humanely acceptable through the use of the Guide. The Guide also later states that institutional animal facilities and programs should be operated in accordance with the recommendations and requirements of the Guide, and that each institution should establish an animal care and use program that is managed in accordance with the Guide. So the idea that the institution is of central importance is 
really driven home. And, of course, this is something that AAALAC and I, as an AAALAC site visitor, considered very advantageous because it had an impact on how AAALAC would do business, as I will explain.

Prior to 1986, during the period when I was involved in AAALAC as an ad hoc site visitor, the site visitors were responsible for creating the program description as part of the site visit report. As the junior member of the site visit teams, I was required to generate the program description. Typically, the institution would provide skeletal information-a few pages of handouts broadly outlining a few basic elements on how the program operated. Then, during the site visit, visitors were expected to accumulate all the information concerning the physical facilities, husbandry and sanitation, program of veterinary care, and so forth, to generate a written description of the program's resources and how the program operated. The program description was followed by the evaluation and finally a letter summarizing the accreditation worthiness of the program. Composing that total report package was a pretty onerous task. (Those of you who now write program descriptions for your own programs know that these are from 30 pages to, sometimes, upwards of a couple hundred pages to describe your institution's program completely.) But with the publication of the new PHS Policy, AAALAC looked for a change of direction. We realized that now that institutional representatives ultimately have the responsibility for institutional obligations, perhaps we ought to saddle them with writing their own program description. It was a wonderful development. [laughter]

What did institutional ownership of the program imply from AAALAC's perspective? One thing it implied was that there were responsible parties at the institution who were, or should have been, in active possession of pertinent information about their program. Further, AAALAC noted that if there are deficiencies in the program, the responsible party at the institution ought to be able to detect them, report them, and resolve them in a timely fashion using internal mechanisms. Thus, the operating style of some programs of that era that was poorly documented, highly chaotic, and libertarian, and required AAALAC to come in and get the program back on track every 3 years was now clearly anathema to both AAALAC's expectations and PHS Policy. The idea was that the institutions needed to take ownership of their responsibility in a very meaningful way.

This had a really important implication for AAALAC because it meant that AAALAC's picky discussion of facility problems — plaster, paint, deteriorated and rusty surfaces, and defective equipment-could largely shift to a more meaningful discussion of institutional preparedness, planning, process, and cooperation.

To illustrate the outcome of this transition in perspective, I want to discuss some of our facility findings from 1986 versus the most recent interval for which we have compiled the data, 2003 to 2008. I started by reviewing site visit reports from 1986, which was a great reminiscence for me since I had written some of those reports. I pulled reports from only two trimesters of the year, since I am a big believer in reduction-if you don't need to do a 20-animal experiment, do a 10-animal experiment. After I had reviewed 86 site visits, I thought I had enough data to illustrate my point. I compared this with data that we presented at the Conference on Quality Animal Care in 2009 in association with the PRIM\&R meeting in San Diego.

I'm sure most of you know the two designations used in AAALAC parlance, a "mandatory" item or a "suggestion for improvement (SFI)," but I'll explain the differences for those of you who don't. A mandatory item is a serious deficiency that must be corrected for full accreditation to be awarded or continued at an institution. These are thankfully getting rarer and rarer in our site visits, and I hope they become obsolete. There are also suggestions for improvement: items that the Council feels are desirable to upgrade in an already acceptable or commendable program.

Table 1 provides a summary of AAALAC's findings pertaining to the condition of animal facilities before the full implementation of PHS Policy and approximately 2 decades later. In 1986 there were 36 mandatory findings among the 85 programs visited, yielding an incidence of 42 per hundred. Those physical plant findings accounted for $25 \%$ of all the mandatory findings in the reports from that era. During the 6-year interval 2003 to 2008, we looked at 1281 institutions and found only 78 mandatory items related to facilities. Comparing the 1986 data to the 2003-2008 data, the incidence of mandatory items fell from 42 to 6 per hundred

Table 1 Compilation of AAALAC's Mandatory Findings and Suggestions for Improvement (SFIs) pertaining to facilities: Pre- and post-PHS Policy implementation

\begin{tabular}{lccll}
\hline Interval & $\begin{array}{l}\text { No. of } \\
\text { institutions }\end{array}$ & $\begin{array}{l}\text { No. of facilities } \\
\text { findings }\end{array}$ & $\begin{array}{l}\text { Incidence of } \\
\text { findings }\end{array}$ & $\begin{array}{l}\text { Percent of all } \\
\text { findings }\end{array}$ \\
\hline $\begin{array}{l}\text { Mandatory } \\
1986\end{array}$ & 85 & 36 & 0.42 & $25 \%$ \\
$2003-2008$ & 1281 & 78 & 0.06 & $16 \%$ \\
2008 & 235 & 8 & 0.03 & $13 \%$ \\
SFIs & & & & \\
1986 & 85 & 110 & 1.29 & Not done (ND) \\
$2003-2008$ & 1281 & 256 & 0.20 & ND \\
2008 & 235 & 35 & 0.15 & \\
\hline
\end{tabular}


programs visited. In the 2003-2008 interval, physical plant mandatory findings accounted for only $16 \%$ of the total number of mandatory findings. In a single-year comparison-2008 was the most recent year in which we looked at this-we site visited 235 institutions and found only 8 mandatory items in the area of physical plant. This represented an incidence of 3 out of 100, and facilities/physical plant problems had decreased to $13 \%$ of all mandatory findings.

In 1986 we found 110 SFIs, and many of these were multipart suggestions; I did not count the individual subparts in this analysis. Table 1 indicates an SFI incidence of 1.29 per program. During the 2003-2008 interval, we identified 266 suggestions for improvement among the 1281 programs site visited. Less than one in five programs had an SFI in physical plant. Finally, in our most recent full-year data, from 2008 , only 35 SFI findings in 235 programs, or less than one in seven programs, received a suggestion for improvement in the area of physical plant.

Let's go over the hot spots in AAALAC's findings in the area of facilities. In 1986, the top issue related to surgical facilities: 24 of the mandatory items related to changes required in such facilities. This issue spanned the era immediately following the implementation of the 1985 Guide. In the 1978 Guide, there was not much of a description of what was expected for an adequate surgical facility, but in the 1985 Guide the requirements for surgical facilities used for aseptic surgery were well defined. Surgery facilities used for USDA-covered species were expected to have separate areas for different activities related to surgery: one for surgical supply support, a preparation area, the operation area, and also space for the provision of postoperative patient care. So some of the functions that were now segregated had previously been frequently conducted in less space or perhaps even consolidated into one room. Needless to say, many of the surgical facilities that were observed in the 1986 site visits were departmentally controlled animal facilities and their design and operation did not reflect current veterinary practices. The second-ranking issue on the findings list was heating, ventilation, and air conditioning problems, and the third was problems in satellite housing areas (i.e., usually departmentally controlled housing areas).

We can compare the trends from 1986 versus the 20032008 period. A comparison to a single year, 2008, makes little sense because there are so few mandatory findings in one year in the present era. In the 2003-2008 data it is evident that we continue to have many programs (39 instances) with problems in heating, ventilation, and air conditioning. I would surmise that in a lot of instances these problems are occurring in some of the older buildings and renovation is costly. Some older buildings have been brought back into use because of expanding animal populations and the need to use all animal housing areas available. So that continues to be important. The secondranked finding involved inadequate provision of viable functional areas (16 instances) needed to meet essential programmatic needs: storage, quarantine, separation of species, isolation of sick patients, and the like. Other mandatory findings in order of declining rank are problems with surfaces in animal facilities (9 instances), temperature and humidity control (6 instances), and last, aseptic surgery (3 instances) still shows up on the radar screen. Physical plant findings are getting much rarer because organizations are attentive and they are participating in central planning in an appropriate way. If you look at what happened just during the 2003-2008 period, it is evident that far less than $10 \%$ of our mandatory findings relate to physical plant. In contrast, more than $60 \%$ of our mandatory findings relate to institutional policies-namely animal care and use committee function or occupational health and safety matters. Had I summarized data for the year 1986 and before, problems with institutional policies wouldn't have even been apparent because there was really no mandate and there were no authoritative guidelines to measure institutional performance.

Obviously there was a great deal of thought and logic put into the development of the PHS Policy of 1985 because it had an extremely profound effect on the behavior of institutions and how they care for and tend to their programs. What has the PHS Policy done for us, in my view? It instigated programmatic development very directly in institutions. It also enabled AAALAC to leverage change in institutions and help them make progress by invoking and reinforcing the concept of institutional responsibility. This permitted AAALAC to point to the fact that the institution is expected to have $a$ program or consistent programmatic guidance and oversight, not multiple, widely disparate programs each run according to the resources, specifications, and/or random biases of a local controlling entity - a department, laboratory, or investigator-that fails to comply with or misinterprets the Guide. Institutions have widely adapted to this approach and have responded with enhanced teamwork among their professionals. I think that accounts for the increasing number of stellar programs and the real rarity of programs participating in AAALAC that are not performing well and have mandatory items found during AAALAC site visits.

I would submit that everyone has really benefited from the paradigm shift to institutional oversight. Certainly, from the standpoint of the AAALAC site visitor there is a sense that institutions are exercising greater control and promptly considering and correcting facilities problems. This lets AAALAC concentrate on other meaningful issues, which really help organizations define and pursue their futures in a sensible way.

One final note related to AAALAC's mission: we are a voluntary accrediting organization. We provide advice and independent assessments to participating institutions, and we accredit those that meet our standards. It should be very obvious that when you're there transiently to provide advice and independent assessments, you cannot be expected to micromanage the program and provide the daily impetus to do things the right way. This is something that has to come from the belief system and brain trust of the institution. I applaud the Public Health Service Policy, buttressed by the Animal Welfare Act, for giving us the tool that was needed to put institutions on the right track. I applaud you as an industry for responding positively to what the PHS Policy expects. And as a leader in AAALAC International, I'm very thankful that the chemistry of compliance has changed so profoundly. 


\section{How We Got There}

\section{Lauretta Gerrity}

t's a great pleasure to be here and to be able to share with you some of the experiences of one institution, the University of Alabama at Birmingham.

I came into the field of lab animal medicine in the late 1970s. Today's presentation will focus on events, particularly at UAB, starting in the mid-90s. We'd [gone] along from the mid-80s on a pretty steady state until the mid-90s and suddenly our animal use took off. For those of you who may not know the term "care days," it's like "hospital beds." We're talking about units: one box of mice kept 30 days would be 30 care days or 30 boxes of mice kept 1 day would be 30 care days. We're talking about millions of care days.

At the same time, we were losing ground in terms of space. We had some new facilities designed but when we projected our use, we'd be falling short, both short term and long term. Several other factors were affecting this besides physical space. We had rapidly growing rodent populations. We had a lot greater use of genetically altered or immunocompromised animals. We had a change in animal models. I'm sure all of you at institutions are aware of this-you recruit a new investigator, recruit a new research team, open a new department, and it's a whole new world. We were having to deal with studies that required better control of lighting, temperature, the use of hazardous agents, as well as the use of magnetic resonance (MRI) and other imaging technologies. We also had out-of-date facilities and equipment. I hasten to add that we were AAALAC accredited and were able to maintain those standards. But subtle issues with the facilities were impacting us. We had a lot of difficulty in maintaining conditions-Chris mentioned the HVAC (heating, ventilation, and air conditioning), and that was certainly something we struggled with. The animal care folks and research personnel didn't like working in some of the facilities. I remember our largest facility had just one stall for women in the rest room. The women didn't have locker rooms and it wasn't a nice place to work.

Having said all of that, we had been able to eliminate rodent pathogens. So we were trying to maintain a specific pathogen-free (SPF) rodent population institutionwide in a situation where investigators were continuing to take animals out of the facility and into their labs. We knew we were in a pretty fragile situation, that we were set up to have a major outbreak of disease. In addition to these factors, we had increasing personnel safety concerns with allergens, as an example. And we needed not only greater biosafety security but also physical safety and security.
How to fund the projects? I got all my data together and went to see my boss, the associate provost. I laid it out; she appreciated the detail I provided, said it all made sense, and said, "We don't have the money." I remembered that there might be federal money available and I thought NCRR might have some grant opportunities. So we thought we would go on the offensive, if you will, and try to educate our administration about what our needs were and show that we were willing, as veterinarians in charge of the animal care and use program, to put forth the effort to try to get some of this outside money. That was going to mean prioritizing our needs and phasing projects and leveraging funding at every opportunity.

This is where we are now: we have put over $\$ 90$ million into this program. That's a huge chunk of change by anybody's reckoning. UAB has put in about half of it. This is pretty surprising when you consider that 10 years ago, when I went to that associate provost, she said the money wasn't available. But they found it. And we've been very fortunate in the federal funding. Our funding comes from many sources. I began to go out and educate people I might get money from, such as department chairs and central administration. And that resulted in money from bonds, money from competitive institutional opportunities, even philanthropic money.

The money from the federal government came from a number of sources. Although I'll focus on NCRR, we were also competitive and secured an award for a regional biocontainment lab, which constitutes most of the new construction money from the federal side (NIAID). We had money from the National Institute of Standards and Technology (NIST) and other sources. So as I said, we decided to partner with NCRR and use every opportunity to apply for CO6 and G20 awards.... We decided early on that we would not miss an opportunity to put in a grant application. You know the saying, "vote early, vote often"? That's more or less what we did. We began by securing an institutional competitive award for caging in a small program. And we built on that for our first C06 application. We've continued through the years to do that, and continue even today.

Let me give you an example of some of the phasing and some of the things we've tried to do. One of our larger facilities was built in the early 1970s around a concept that was very popular at that time: there was a clean corridor that fed out into a dirty corridor that led back to the cagewash. They subsequently added three sections that were not consistent with the clean-dirty corridor plan. So this is a facility that 
had been added on to over several years. The corridors are pretty narrow. It's hard to get from one place to another. They had carved out a section to handle a transgenic animal facility, which effectively cut off a corridor. When I arrived in the '90s, everybody was cutting through the animal facility to get from one place to another.

After four phases of projects and almost 10 years of effort, we've relocated the cagewash toward the loading dock, which is now a dedicated loading dock. We've moved from one room for surgery to a whole suite as well as an imaging facility. We've moved from corridors to suites. And all without losing animal housing space.

Of the facilities that have been the subjects of some of the federal funding, one is new and one is 40 years old. Now investigators and personnel don't hesitate to work in either one of them. The corridor of the new one has windows that let in outside light. In the older facility, which is underground, we put windows inside to help open it up.

We've added procedure space. The surgery was originally one room and we had plans to expand on that with the renovations but about the time we were putting together the plans, we learned that the department of surgery was planning to build a whole new suite of its own. So we went to them and suggested that it might be the best use of everyone's resources if they would let us try to accommodate their needs. We got several of the surgeons involved in the design of the facility. The hospital was undergoing major renovation at that time, and one of the surgeons said, "I don't know what they are doing with all those old surgery lights." You know where they ended up? A good bit of the fixed equipment for [the lab animal] surgery came directly from the human surgery. The surgeons like working in here because it's equipment that they are familiar with and they helped design it.

The renovations also gave us an opportunity to put in place a lot of rodent surgery procedure rooms, so people didn't have to take animals out of the facility any longer. Another opportunity was to begin teaching people to use gas anesthetics in rodents, to intubate them or use masks as opposed to using injectable anesthesia. I think that we now have over 70 gas anesthetic machines in use around campus and... that they've improved animal welfare quite a bit.
In summary, we've got better use of space. We've got better control of allergens, infectious agents, and other hazardous agents, because people do not have to remove the animals from the facility. There is easier access to the veterinary staff because they're right there. There's easy access to equipment and supplies. And it has certainly facilitated oversight by the IACUC. There's enhanced personnel safety. We now have change stations and biosafety cabinets for all rooms or suites and downdraft tables in the necropsy rooms for both large and small animals. We've reduced security concerns in other ways. Post-9/11, there's been increased focus on securing cesium and cobalt units. We have moved to X-ray irradiators that require much less security. All these have been purchased in cooperation with NCRR funding. There are better sanitation facilities. We also have purchased a water bagging system that allows us to produce a thousand bags per hour for rodent cages. This is important for us, being in the south, where we are subject to hurricanes. Now our emergency water plan is to store up the bags instead of putting water in clean trashcans. And we now use a hydrogen peroxide sterilizing unit instead of formaldehyde for disinfecting rooms.

The caging is an interesting story. We had managed to get filtered tops on all the mouse cages, but we were inappropriately using the top shelf of racks because we didn't have any other place to put these animals. In trying to figure out how to use our money most efficiently, we knew we had to replace a certain percentage of our cages on a yearly basis. We decided that we would begin replacing cages with a style that would allow us to use the ventilated racks. Our grant applications to NCRR described this plan. NCRR bought the racks and we bought the cages. This worked out well. We put these cages and racks into use by colony and then by building. We started with the mice and now all of our mice and our rats are in ventilated cages.

To leave some parting thoughts-I think of these as "duh" moments. It's obvious that meeting the animal research needs of investigators is an ongoing process. It's changed in the 10 years that we've been working and it will continue to change. Animal facilities to support research require significant investment in infrastructure. There must be a commitment on both the part of the institution and the funding agencies to ensure animal welfare and good science.

\section{Animal Facility Improvement Project Funding}

\section{Willie McCullough}

he goal of this presentation is to answer two questions: Is there a current need for additional animal facility space in the biomedical research community? What contribution has NCRR made toward improving animal facility space in the nation's biomedical research institutions?

In the mid-1980s both the House and the Senate brought in experts to discuss the seriousness of conditions of the 
nation's biomedical research facilities. As a result of those expert testimonies, the National Science Foundation was mandated to collect and analyze data on science and engineering research facilities.

In fiscal year 2005, the National Science Foundation surveyed deferred animal research facilities projects-those that need to be done or are on the books to be done, but for which the funding source or stream is not available. The 2005 survey data, published in 2007, showed $\$ 9.8$ billion in deferred biomedical research construction projects, with $\$ 1.24$ billion in deferred animal research project space. Fiscal year 2003 animal research space survey data revealed $\$ 0.73$ billion in deferred projects. Thus there was approximately a doubling in the deferred animal research space between 2003 and 2005. This confirms the need for the existence of the NCRR animal improvement program.

NCRR has administered an animal facility improvement program since the 1980 s. The program provides grants to upgrade existing animal facilities including diagnostic laboratories, transgenic animal resources, and similar activities to support biomedical and behavioral research; to support alteration and renovation to laboratory animal facilities; and to purchase equipment. The program is currently active, with funding in the \$7-8 million range each fiscal year. The maximum requested amount is $\$ 500,000$ for renovation and equipment costs directly related to the care of research animals. There is no minimum amount that may be requested; if an applicant needs $\$ 100,000$ to improve an animal facility, that amount is acceptable. Five years are allowed to complete the project after the award is made. Approximately 16 awards are made each fiscal year from a pool of 80 to 100 applications. In addition to upgrading the animal facilities, these grants assist institutions in complying with the Animal Welfare Act and DHHS policies related to the care and use of laboratory animals.

In 1991, there was approximately \$11 million in funding for the program (the funding level tends to vary from year to year). During the NIH budget-doubling time frame, 19992003 , the funding level for the program was about $\$ 12$

Table 1 Top ten states that received animal facility improvement awards (FY 1989-2010)

\begin{tabular}{ll}
\hline State & $\begin{array}{l}\text { Number of } \\
\text { awards }\end{array}$ \\
\hline California & 56 \\
New York & 49 \\
Pennsylvania & 36 \\
Massachusetts & 33 \\
Texas & 32 \\
Illinois & 21 \\
Oregon & 21 \\
Louisiana & 18 \\
Washington & 17 \\
Ohio & 16 \\
\hline
\end{tabular}

\section{Box 1 \$1 Billion Recovery Act (ARRA) Construction/Renovation Initiative}

- 1200 applications were received in FY 2009 for C06 construction ( 75\%) and G20 core facility ( 25\%) projects

- $\$ 10.6$ billion requested (40-50\% for animal facilities improvement projects)

- $\quad \$ 1$ billion funded 147 awards (87 C06 and 60 G20 core facility improvement projects) to institutions in 44 states, the District of Columbia, and Puerto Rico

- Approximately $12 \%$ success rate

million; current funding is \$7-8 million each fiscal year. From 1989 to 2010, NCRR made approximately 560 animal improvement project awards. In fiscal year 2010, 15 awards were made.

Table 1 shows the top 10 states that received G20 animal improvement awards. California is the big winner, with a total of 56 awards, and Ohio is tenth with 16 awards. What is important is that the 10 states listed received $53 \%$ of the 560 awards. Three states-Wyoming, Delaware, and New Hampshire-have not received an award through this program.

A second NCRR program has made a tremendous contribution to improving animal care facilities, and that is the C06 Extramural Construction Program. This program was funded from 1994 to 2005. During those 11 years, NCRR made 340 awards for $\$ 660$ million, which was leveraged to $\$ 2.2$ billion in improvement projects through institutional matching funds. Animal facilities throughout the country benefited from this program. Each C06 improvement project must be used for biomedical research for 20 years. For example, a project started in 1994 is allowed up to 5 years for completion of the project in 1999 . The completed project space must be used for biomedical research for 20 years after occupancy. This means that those early 1994 awards have about 10 years left in their 20 -year use requirement. The last time this program had funding was in 2005, which means that those projects were completed at the end of fiscal year 2010 and began their 20-year use requirement.

In 2009, the C06 extramural construction program received \$1 billion in Recovery Act funds (Box 1). NCRR received 1200 applications, 75\% of them for C06 construction projects and $25 \%$ for G20 core facilities. It is important to note that both the $\mathrm{C} 06$ extramural construction projects and the G20 core facilities included animal facility projects. The \$1 billion funded 147 awards, 87 for C06 construction projects and 60 for $\mathrm{G} 20$ core facility improvement projects. These awards went to 44 states, the District of Columbia, and Puerto Rico.

Both the G20 and C06 programs continue to play an important role in the improvement of animal facilities at biomedical research facilities throughout the nation. 


\section{Where We Are Today}

\section{Steven Leary}

couldn't comment about where we are today or where we will be in the future without thinking about the past. In a photo from the Wistar Institute in Philadelphia, animal caretaker Fannie Louise Duhring performs her duties in street clothes (with jewelry), no personal protective equipment (PPE) in evidence (Figure 1). The animal holding room itself had the supply grills in the ceiling and the exhaust grills underneath windows leading directly to the street outside. The cages were wood, likely unsealed. A single changing table sat in the middle of the room. The cagewash was equipped with a large tub in the middle of the room and a dryer along the back wall. The overall facility floor plan placed the lavatory next to the surgery with an office next to that. This is not all ancient history-I can remember performing AAALAC site visits where infection control consisted of sticky mats outside the facility door, and working with scientists whose animal colonies were affected by common pathogens to the extent that it was impossible to generate accurate, reliable research data.

The increasingly sophisticated science we support necessitates the provision of equally sophisticated animal care. Research today is affected by complexities such as unknown agents (e.g., norovirus), molecular genetics, impacts of knockout and knock-in genes on a particular strain, and any combination thereof. Animal facility design can mitigate (or aggravate) these factors.

The primary objectives in designing and building today's animal housing facilities are to satisfy overall institutional needs and meet animal needs while being user friendly for both husbandry and research staff. We need to be flexible in our designs for multiple rodent species, nonhuman primates, and large domestic animals. Occupational health and safety are important, as is the general work environment. Regulatory compliance must be considered while still being operations and maintenance friendly. We need to provide increased security and effective building management systems. And we must be cost effective.

Achieving these goals is affected by a number of trends and issues. One of these is animal census growth and projection. NIH budgets are critical to animal-based research grants. Many institutions are in a $40 \%$ to $55 \%$ range of their grants using animals; some are at $60 \%$. Animal health status needs to be more tightly determined and controlled with quarantine. Operating costs and per diem reduction are always important and can be impacted by centralized versus decentralized location of facilities. Automation, rack and husbandry protocols are also very important. Imaging is increasingly used. Sustainability has become important-NIH has added it to grant criteria-and can
Figure 1 Animal caretaker Fannie Louise Duhring performs her duties in street clothes and jewelry, ca. 1931. Photograph used by permission of the Wistar Institute Archives.

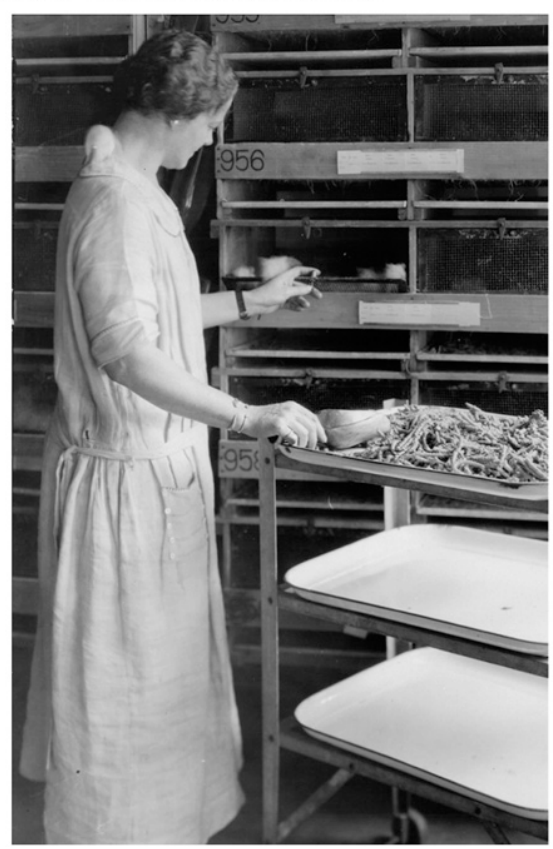

be improved with air change per hour approaches in rooms, ventilated racks, and improved types of support equipment.

Planning ratios, or the number of animal holding rooms to procedure rooms, is one issue for planning and programming. Impacts of the new Guide for the Care and Use of Laboratory Animals must be considered. Occupational health and safety concerns such as ergonomics and allergen control have to be taken into account. Increasing numbers of biohazard studies, as well as national, international, and interinstitutional collaborations, make internal and external quarantine an important operational consideration.

Cores need to be considered in animal facility design: transgenics, rederivation, necropsy, surgery and minimally invasive surgery, diagnostic laboratories, high-throughput behavioral cores, radiation, biohazards, aquatics (zebrafish and Xenopus), and, again, imaging.

As facility designers, today we are better able to meet the needs of the institution. One institution has an imaging core for small and large animals tied with needs for and use of some of the human equipment for MRIs, mass spectrometers, cyclotrons, and microversions of several of those. Another institution's behavioral core uses a large number of small 
Figure 2 Partial interstitial space. Graphic by Jacobs Engineering; used by permission.

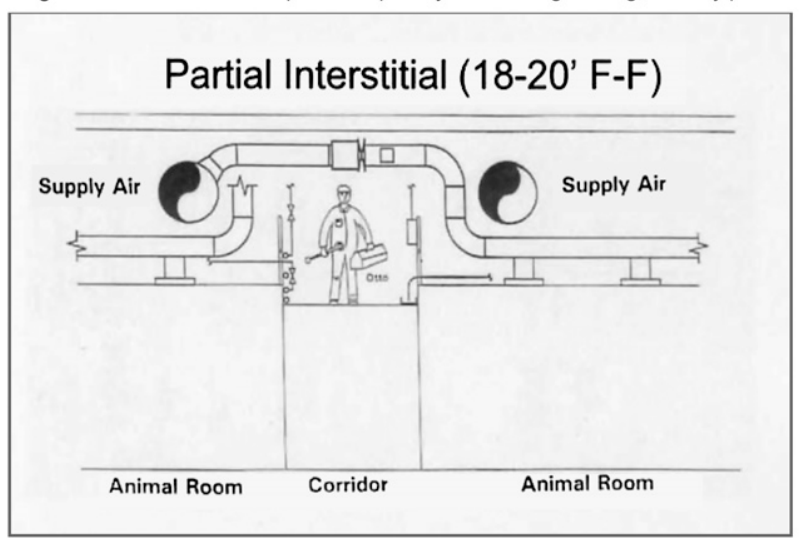

rooms. A facility that houses mostly nonhuman primates has rooms that are larger in size and fewer in number. Multilevel, dual corridors, expandable barrier and nonbarrier animal rooms, a multilevel building with support space in the subbasement and first floor with a single double-loaded corridor-these facilities are designed based on the research needs of each individual institution. ${ }^{1}$

Most new buildings, especially steel building construction grids, are on 22 -foot centers. This enables design of modular animal rooms, 22 feet wide or $101 / 2$ to 11 feet wide. With each of those, you can define for the institution the projected animal caging census in that room, the cage density for that area, and the rack equipment cost for each room. This allows for large double rooms holding 800-plus cages or single rooms with half as many cages (420). These can be library style or single-sided racks along the walls.

Ventilated racks have been available for many years, and the construction technology has continued to improve. There are a number of installation options, including self-contained blowers, hooking directly into building supply and exhaust systems, iris dampers, and phoenix valves. Both supply and exhaust are important to sustainability-decreasing the number of room air changes needed decreases tonnage in the HVAC system.

Each institution has its own opinion on procedure rooms. Institution A has one room in its suite of almost 1700 cages, while Institution B has one for about 1200 cages. Institution C wants more procedure space within the barrier, so it has one procedure room for 500 cages. Procedure room design is

${ }^{1}$ Technical information, floor plans, photographs, and graphics for the original presentation and referenced in this article were provided by Jacobs Engineering, Tecniplast, and Sigma Engineering and used by permission.
Figure 3 Laboratory automation. Photo by Tecniplast; used by permission.

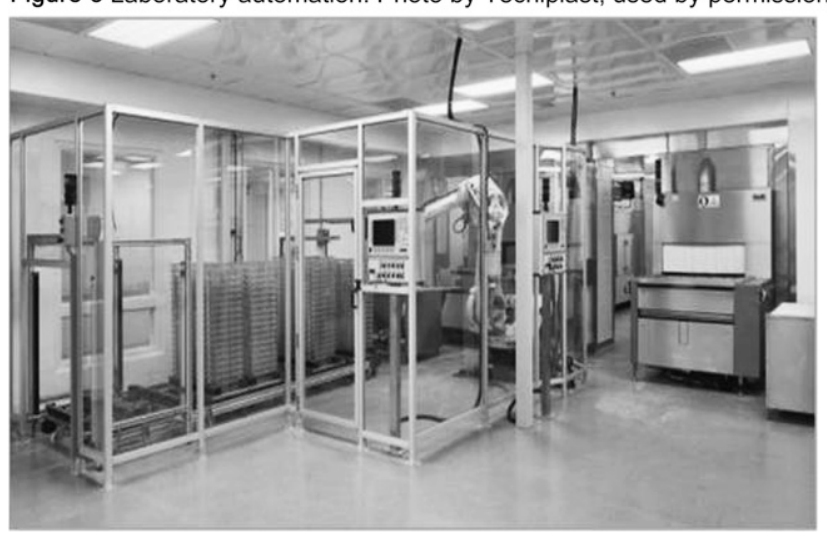

dependent to some extent on the research program, but is always driven by the attitudes of the institution.

Disposable caging is being evaluated at some institutions for both centralized and decentralized facilities. It decreases upfront capital costs by eliminating the need for cagewash.

Interstitial space can also enhance facility design flexibility for future needs. Partial interstitial space (Figure 2) will add about $\$ 7$ per square foot and full interstitial space about $\$ 25$ per square foot to initial construction costs.

Automation is becoming much more popular (Figure 3). It adds considerable operational efficiency (decreasing personnel as much as two-thirds), is beneficial for allergen control, and increases personnel safety. In cagewash, robots can dump soiled bedding and dispense clean bedding. There are vacuum systems, bottle handling systems, and radio frequency identification (RFID) caging. Automation can be used for single-sided (clean or dirty) or double-sided (clean and dirty), and can be duplicated for larger facilities. There's a new system that inverts the cage as it comes off the tunnel washer, and another that will do entire cage setups and place them back on the rack.

Industrial engineering can assist with flow analysis. For example, where are the most efficient placement locations for waste disposal carts? Automated guidance systems can be programmed to move carts to these dump stations, freeing personnel for more important tasks.

Looking to the future, there are a number of scientific and technological advances that may eventually affect research animal use-for example, biological computers, synthetic biology, and nanotechnology. Nanobiosensors can be placed in mouse cages and read through Wi-Fi.

As we move forward, we must keep in mind that although new technology is fascinating and important and may help improve our science, our major goals as laboratory animal medicine professionals are personnel safety and animal welfare. 


\section{Animal Housing Facilities: Discussion}

We now have time for questions and comments.

Questioner: I want to say a few remarks because I think it's important that we not lose sight of the full historical picture, and that's really critical as we look over the agenda today and tomorrow. I greatly appreciate the commentary from people today. I did [bring] a few historical documents, which I'd be happy to make available to those who are interested: Beyond the Laboratory Door, which was published in the mid-1980s, and a brochure that was part of the campaign trying to get the 1985 Amendment to the Animal Welfare Act passed over the strenuous objections of the medical research community.

USDA has certainly come a long way (I appreciate Dr. Goldentyer's remarks) in moving from Brucellosis testing to enforcing the Animal Welfare Act in research labs. And inspectors have certainly had to endure a lot. Some were overwhelmed, perhaps, by the task at hand and trying to locate the animal facilities of satellite facilities or the University of Pennsylvania's Head Injury Lab down in a basement....

The problem is that I don't think the animal welfare community is represented on the program.... I think it's important to understand the welfare perspective from those who were really the strongest, most strident activists to seek change in the laboratory, [who] supported animal research but really pushed hard to bring change-moving from small barren cages where animals were singly housed, facing arguments about historical control where animals have always been housed in small barren cages.... And then ultimately seeking performance standards.

When it came to enforcement, the inspectors didn't have a clue what to do and USDA's own survey of performance standards shows they didn't know what to do. Which was exactly what certain representatives wanted to have happen. When we wanted improvements in primate care, it was mandated in 1985. Changes had to be made allowing socialization for primates, for example, and other enrichments. [We were] told we were anthropomorphizing.

[But] changes have occurred.... Delighted to see the NIH...is embracing refinements - things like teaching animals to cooperate for procedures, to reduce variables to improve the science,... [like] quality care that's being provided by animal technicians....

My point is, we have to look forward now, by understanding the past and look at what progress has been impeded by the stumbling blocks and how we're going to move from here. In 1985-25 years ago_-we passed a law calling for a physical environment to promote psychological wellbeing of primates. As of the most recent survey that I'm aware of, a few years ago, only $46 \%$ of primates housed indoors are socially housed. Social animals_rats, mice, and birds - are removed from the law. Yes, they might be covered in part by NIH, although they are not inspected. Yes, they might be covered in part by AAALAC every 3 years. [But] how much protection is there? And how about the small colleges where no one sees the rats, mice, and birds? What about the private institutions where, again, no one sees? Are we going to work together to address these problems or are we going to try to push them away? Class B dealers: NAS does a study, the result demonstrates there is no scientific need for Class B dealers, [but] NIH hasn't let go of that yet. What are they doing to make it happen? They're dragging out the time period to implement that. The...facility is doing the minimum to make it happen.

The question is, What are we going to do to help more be done for animals? I hope that we can work together to make this happen....

Questioner: I'm on an IACUC [and] we're beginning to hear some questions, mainly from exercise physiologists, those types of researchers, that our current housing standards in terms of space for laboratory animals are grossly inadequate. They cite data, especially in some longer-term experiments, that show even the control animals are showing signs of inactivity disease, metabolic disease, diabetes, and so forth, compared to their wild counterparts, housed in their natural habitat. So the question is to any of you, maybe all of you, how do you see addressing this issue? Where do you see us going? Do you see us as going to a situation where we will house rats and mice in holes in the ground? Rabbits in the bushes, out in open spaces for experiments? Just your opinion?

Dr. Bennett: What species in particular?

Questioner: Mice, lab animals that are housed in cages.

Dr. Bennett: I don't think we'll be going back to naturalistic environments because you're going to lose some of the control that animal facilities were designed to implement into the process. There are some papers out now on how we assign animals to various groups. Joe Gardner did a very interesting presentation at a recent AALAS meeting. I think we need to relook at how we assign animals to groups to take out intercage variability. And so I think we could handle some of those issues by doing that.

Questioner: [The first questioner] expressed a lot of the things I was planning to say so I appreciate what she said and all the work she did on the Hill to make these changes happen. It's extremely disappointing that nobody from any animal protection organizations is on any panels here or was approached to be. I think that's a huge mistake. And just seeing the panel this morning shows that we're still missing the point. We've understood, now, how much money facilities are getting for construction and how there's automation, so it's easier 
on people. But we didn't really hear about how housing has changed for the animals - and largely, it hasn't. If you show a picture of a primate cage from 40 years ago and a primate cage now, it's basically the same: it's all metal with a perch added. I'm very disappointed that we didn't learn more about what's happened for the welfare of the animals since 1985 and that we weren't invited to be a part of this discussion.

Questioner: I have a question for Dr. Newcomer. With AAALAC's expansion globally over the last 10 to 15 years, and more and more facilities being accredited outside North America, is there any difference applied to the standards in those facilities and is the new Guide being implemented when you do your inspections at this time?

Dr. Newcomer: AAALAC applies the same standards globally. A recent change is that we are now looking to the new Guide.... It's one of three documents that are important in the accreditation process: (1) the $8^{\text {th }}$ edition of the Guide, when it's finalized and when we work through the issues related to that; (2) the European Convention for the Protection of Vertebrate Animals used for Experimental and Other Scientific Purposes (ETS 123) from the European Treaty Series-it's an appendix to the European Directive [and is] considered an important guidance document; and (3) the Guide for the Care and Use of Agricultural Animals in Research and Teaching. So there are three primary documents that are applied in concert and used internationally in all the accreditation visits.

Questioner: Is that next year, after it's finalized?

Dr. Newcomer: We're anticipating that when [the new Guide] is released, in final form, we'll be able to include all of the frequently asked questions and position statements that we need to put out into the community for comment and as an advisory to the accredited community so they can interact with us and understand what they will need to cope with during the transition. And we do imagine that, realistically, that will happen during 2011. 


\title{
Institutional Animal Care and Use Committees: Session Introduction
}

\author{
James Taylor
}

or those of us involved with the Department of Defense (DoD), 40 years ago the DoD implemented local committee oversight and approval responsibilities over research activities. Twenty-five years ago, as you have been reminded, USDA and NIH implemented major revisions to their regulations and policies, respectively, to create what we now know as animal care and use committees. Our three panelists will cover the past, present, and future of the multiple activities these committees perform to advise and support the humane and appropriate care and use of laboratory animals.

Our first speaker is Dr. Ralph Dell, who is now a private consultant. Previously he served as Director of the Institute for Laboratory Animal Research at the National Research Council and as Chairman of the Institutional Animal Care and Use Committee at Columbia University. Dr. Dell re- ceived his MD from the University of Pennsylvania and is board certified in Pediatrics. He is a Council member emeritus for AAALAC International.

Our next speaker, Dr. Kathryn Bayne, is the Global Director for the Association of Assessment and Accreditation of Laboratory Animal Care International. In this role, she directs the accreditation program worldwide, traveling extensively to advance AAALAC's accreditation program and laboratory animal welfare. Prior to this position, she worked at the NIH leading a research program on nonhuman primate psychological well-being and environmental enrichment programs for primates, cats, dogs, and swine. She has published over 40 articles on the subject, is a certified animal behaviorist, and is internationally renowned for her work in laboratory animal behavior. She has also published extensively on accreditation of laboratory animal programs.

\section{Formative Years}

\section{Ralph Dell}

'm looking forward to sharing some of the horrendous experiences that we went through at the College of Physicians and Surgeons at Columbia University in late 1985. This followed the publication of the Amendments to the Animal Welfare Act.

Before I get heavily involved in Columbia's experience, how did I get involved in this? Well, I was a professor of pediatrics and had a fairly sizable research lab. We carried out a series of experiments in dogs studying acid-base metabolism trying to understand acid-base disturbances that afflict newborn infants. The idea was to try to devise therapies for such disturbances, and the results of this research led to better detection and treatment of them. We also developed a solution for the intravenous feeding of prematurely born infants. The animal models we used in this research were guinea pigs, mice, and random source dogs, and the work resulted in the commercial development of a solution for the intravenous feeding of prematurely born infants or, in some cases, older infants that were not gaining weight normally.
Because I used many animals in my research, I was asked, rather casually, by my department chairman, to be on the animal committee. He said, "It won't require much work. It's just a brief meeting every month or two." Sometimes life's changing events come in deceptive packaging.

As I mentioned earlier, there were several changes in the regulations governing the use of animals in research. The PHS Policy was updated in the same year (1985) as the publication of the Amendments to the Animal Welfare Act and the publication of the US Government Principles. Part of the impetus for all of this activity was the publication of the International Guiding Principles for Biomedical Research Involving Animals by the Council for International Organizations of Medical Sciences (CIOMS). This report was the result of meetings between 1982 and 1984. In the report, CIOMS noted that animal research is fundamental to the biomedical sciences to improve prevention, diagnosis, and treatment of disease in both humans and animals. Recall that the AIDS epidemic had started and there was considerable interest at 
the time, and still is, in developing an animal model of AIDS. Publication of the CIOMS report spurred an update to the PHS Policy to ensure congruence with the CIOMS Guidelines. Given the changes in PHS Policy, NIH asked funded institutions using animals in research to provide a description of their animal care and use program.

This request was received by our grants and contracts office in late November at a time when everybody was in a holiday mood. I was the newest member of the animal committee - that's not good - and, unluckily, I was the only one in town when the office of grants and contracts received the request for our description of our program. With only 3 weeks' experience on the committee, I did my best to describe what our program was - and wasn't. I relied on two senior supervisors and a quick walk through the facility. I visited parts of the facility that I hadn't even known existed. One thing I learned was that the USDA regularly inspected our facility. (My only experience with the USDA was as a 6-year-old child accompanying my grandfather who worked for the Department of Agriculture as he visited farms in Southern California. I don't remember what the inspections were all about, except that people seemed quite nervous when we showed up.) There was quite a sizable file of USDA reports that I didn't bother to read. Clearly, we were a research facility, not a farm. The dean signed off on our description, we sent it off, and started the Christmas holiday.

I actually had no idea what it took to have an approvable animal care and use program and I think that deficiency was apparent to the OPRR committee. In mid-January, I received a call to come to the dean's office to be introduced to Gene New and several others who had come to see our animal care facilities and to review our program. Two days later, the site visit team met with the animal committee (which, by the way, shortly became the IACUC). Together with senior officials in the dean's office, we received the news that the team was recommending that our animal research program be suspended pending substantial renovations and development of a comprehensive program of animal care and the hiring of a trained laboratory animal medicine veterinarian.

One of the site visitors suggested that I might read the Guide. My response was, “There's a guide?" You can see that I was starting from minus zero. The site visitor gave me his copy of the Guide to look at. I thumbed through it, reading the chapter headings, and immediately ordered 20 copies from the NIH and distributed them to the animal committee, which, after reading the Guide, we renamed the IACUC. I also gave a copy to several senior investigators and the dean's office.

Recall that I said that I was candid in my assessment of our facilities in December. That candor was acknowledged by Charlie McCarthy in the letter we received from OPRR restricting our Animal Welfare Assurance. In the final paragraph, he says, "It is my judgment that, since the Health Sciences Division of Columbia University [HSDCU] frankly stated these deficiencies in the Animal Welfare Assurance it submitted to OPRR on December 12, 1985, the deficiencies do not constitute a breach of trust on the part of HSDCU."
Part of the reason for my candor was, Don't lie to the feds. And also maybe it would be helpful in raising funds. I'm a little less naïve now.

We first hired a veterinarian, a primate specialist, from the pharmaceutical industry, who oversaw much of the physical plant renovation. But we needed an experienced laboratory animal veterinarian and ultimately hired such a person. We had an architect draw up plans for the renovation and put it out to bid. And again because I lived closest to the facility, I led several of the bidders through the facility. One Saturday morning, after a walk-through, a bidder looked at his notes and said, "I guess I have everything —oh, no, one more thing. What's the cost of doing business in Washington Heights?" I didn't have a clue. It turns out to be the size of the expected donation to the local precinct to avoid having any parking fines as workers come for the project. I'm getting a real education here.

There were several events that led to an inadequate animal care and use program. First, as I mentioned, there had been regular inspections of our facility by USDA. These inspections consumed about 2 days of the inspector's time and the resulting reports were carefully filed by the animal care staff. I never saw them until well after the extensive renovations of our facility had started. One inspector had written that rodent control in the feed storage room was inadequate. In response to this, one of the animal care staff let loose some cats in the feed room. That took care of that problem. But it led to other undesirable consequences.

There were numerous housekeeping deficiencies-wooden doors that needed repair and in some cases couldn't be closed at all, roaches all over the place, et cetera, et cetera, you get the picture. It was really bad. I was pleased that all of these reports were so carefully filed. But I didn't understand USDA's oversight. After all, we weren't a farm. In any case, it would have been useful for at least one or two of the committee members to have read the reports before filing. After we were up and running again, we assigned a person to read the inspection reports and keep the committee up to speed. And to be honest, it did alert us to some problems that needed to be addressed.

This [critique of] our animal care and use program resulted in the hiring of Dennis Cohn as our laboratory animal veterinarian, extensive renovation of our physical plant, costing nearly $\$ 2$ million, and the creation and education of the IACUC. I personally came down to Washington and attended sessions that NIH held to introduce investigators to the new role of the IACUC and the more rigorous oversight of animal care and use programs. This was, however, only the beginning. Dr. Cohn was very instrumental in educating the IACUC and the principal investigators.

As chair of the IACUC, I received visits from many irate investigators who wanted to know what I was doing to get our research program going again. Why was it taking so long? One of the visitors was chair of the Department of Surgery - and the chair of the Department of Surgery at almost any medical institution carries an enormous amount of weight — who was especially incensed because animals 
were used not only in research in his department but also, of more immediate need, in the training of first- and secondyear surgical residents. Also note that there is nothing like being part of the power of the process needed to get us to a place where we could pass a new visit. The animal care facility was given two floors of one of the new research buildings, as well as the whole top floor of our new, and major, research building.

We needed a cage washer, so I went to the dean's office and he said, "OK, what's it cost?" I named the figure-I cannot remember what it was-and the response was, "Is that all? Buy two of 'em and the vets will decide where they have to go."

I had a regular lunch meeting with the vets, a representative of the contractor doing the renovation, and the head of the grants and contracts office. Since I had been at the medical school for over 20 years, I knew how to expedite repairs and installations of new equipment.

The physical plant was only part of the problem. How do you get the IACUC up to speed? What does the protocol form need to ask? Enough to ensure animal welfare but not too much to be a barrier to research. How much justification of a new project is needed? How much of a literature search do you need to ensure that there's no unnecessary duplication?

You can imagine all the meetings that this required, not only to manage deciding how often we needed renewals. I spent hours meeting with department heads, speaking at faculty meetings, and so forth. The job did not-underline not-increase my popularity. When I walked into someone's lab, everyone froze and silence fell. I either skipped lunch because I would be besieged by angry faculty members or I brought in lunch.

Finally, it practically took an act by the dean to get committee members. I had more difficulty recruiting members than the institutional review board (IRB) did. What do you look for on a semiannual inspection? It finally came down to,
Do you want to use animals in research? Then you must follow the Guide. There are no musts in the Guide, right? Well, the Guide may be written to allow professional judgment, but you are not a veterinarian. The ultimate threat that I had was another shutdown and that was a very effective threat.

After things were up and running, protocols were being processed in a timely manner and investigators began to understand what we needed on the protocol form, [and] things began to run more smoothly. After several months of being back in operation, people even began to ask us for help in designing the animal care aspects of their research projects. It took 6 months to renovate the facilities, hire and train animal care staff, and meet with the investigators and staff to emphasize proper care of the animals-for example, no it is not $\mathrm{OK}$ to put out sticky traps for feral animals. By the end of the 6 months, I had practically memorized the Guide.

Also we built a very good office staff, which was invaluable. When the dean asked me to take on the animal care committee and the renovation, I said I would agree to do it if he provided me with two people for the IACUC office staff. "OK," he said, but then almost reconsidered when I asked for his deputy office manager and another one of his top aides.

I also was promised $\$ 1.7$ million to bring the facilities up to snuff. There are some advantages to being shut down. That said, I don't recommend it. I do recommend training people to use animals properly in justifiable research. I do recommend getting AAALAC accredited, a good way to show that you operate a good animal care and use program. It's hard to achieve compliance if you don't have the backing of the top institutional official.

One year after all this drama, I was asked by James Taylor to go on an AAALAC site visit. This led to increasing involvement with AAALAC and ultimately to being a member of AAALAC Council for several years. And finally, I worked for 3 years as Director of ILAR.

There is one thing I can say about retirement: I no longer have the Guide memorized.

\section{The IACUC's Impact on Advancing Best Practices and the Three Rs}

\section{Kathryn Bayne}

\section{Introduction}

$\mathrm{n}$ the decades since the publication of the 1985 edition of the Guide for the Care and Use of Laboratory Animals, passage of the Improved Standards for Laboratory Animals Act, and issuing of the US Government Principles (page 551)_all of which reference the establishment and describe responsibilities of the institutional animal care and use committee - the positive impact of the IACUC on research animal welfare and the quality of science has been significant. Over time, the IACUC's scope of oversight and level of responsibility have increased. Protocol review has intensified, the semiannual program reviews and facility inspections conducted by the IACUC are more detailed and structured, postapproval monitoring systems have been established at many institutions, formal mechanisms have been 
established for the IACUC to receive reports of concerns, and the IACUC's role as gatekeeper in promoting high-quality science at the institution, while being sensitive to societal concerns about the use of animals and promoting institutional self-correction of program issues, has achieved widespread success.

With the expanding role of the IACUC comes a need for enhanced infrastructure and support for the committee to function successfully. This is being met, in part, by the variety of IACUC-specific conferences hosted each year; highquality books and journal articles that explore the various aspects of the committee's responsibilities; and dedicated support staff, many of whom have achieved professional certification. Critical to the committee's ability to fulfill its regulatory and moral responsibilities are IACUC member training, adequate fiscal resources, and sustained and visible support from upper administrative officials. As the role and function of IACUCs have matured over the years so, too, has the committee's ethics-based decision-making process. In partnership with scientists, IACUCs have encouraged the use of less painful methods such as in vitro tests, imaging technology, computer modeling, and the establishment of humane endpoints; they have ensured that staff are competent in performing procedures; they have closely evaluated the justification of animal numbers used; and they have encouraged innovative refinements in animal housing and procedures.

\section{The Three Rs and Ensuring Best Practices}

According to www.businessdictionary.com, best practices are "methods and techniques that have consistently shown results superior [to] those achieved with other means, and are used as benchmarks to strive for." This source notes, however, that "there is...no practice that is best for everyone or in every situation, and no best practice remains best for very long as people keep finding better ways of doing things." Thus, for institutions to ensure that best practices are employed in both the care and use of animals there must be a cultural acceptance that ongoing review of procedures and practices is necessary and that animal care and use programs are not static but continue to evolve and change. Using the descriptors in the newest edition of the Guide for the Care and Use of Laboratory Animals (NRC 2011) for the Three Rs, this change will include replacement techniques, through which the use of animals will be avoided; reduction strategies, in which similar amounts and types of information can be obtained from the use of fewer animals, or the amount of information obtained from a set of animals is maximized; and refinements that involve modifications to husbandry practices or experimental procedures that will improve animal well-being, including the minimization of pain and distress.

The IACUC's role in promoting the Three Rs and best practices is partially accomplished by methods described in the PHS Policy on Humane Care and Use of Laboratory Animals (1985, rev. 2002). Specifically, the Policy requires a semiannual IACUC review of the institution's program and inspections of the facilities using the Guide as the reference point. The reports of those reviews must be provided to the Institutional Official, who is ultimately responsible for ensuring that animal care and use conform with applicable regulations and policies.

Over time, both the breadth and level of detail of concerns the IACUC must attend to have increased. For example, the 1985 publication of the Guide contained approximately 10 pages focused on the discussion of institutional policies, of which a very small portion addressed the function of the IACUC. At that time, the Guide stated that "An institutional animal care and use committee should be established to monitor the [animal care and use] program." The committee was encouraged to meet at intervals appropriate to the institution's program, but not less than annually. In 1996, the Guide contained approximately 19 pages describing elements of the program that should be covered by institutional policies, and in the new $8^{\text {th }}$ edition the content related to this subject expanded to approximately 27 pages.

For 45 years AAALAC International has reviewed and accredited institutional animal care and use programs. This places AAALAC International in the unique position of being able to look at these program improvements over the long term and to witness changes in IACUC functioning. For example, the enhanced oversight of animal care and use programs by IACUCs is evident in the increasingly intense review of proposed animal work exemplified by the inclusion of statisticians on many committees, routine inclusion of the principles of the Three Rs when conducting protocol reviews, and accessing resources such as the Animal Welfare Information Center (AWIC) and the Center for Alternatives to Animal Testing (CAAT) to aid in searches for alternatives to painful or distressful procedures. IACUCs have intensified their semiannual program reviews and facility inspections, and many have additional postapproval monitoring systems in place. IACUCs take very seriously their responsibility to self-report adverse events or issues of noncompliance, thereby fostering a culture of self-identification and correction of issues. In response to federal requirements, IACUCs have established mechanisms to receive reports of concerns involving the animal care and use program so the committee is able to launch an investigation into the allegation and respond appropriately.

This enlarged scope of responsibilities of the IACUC has necessitated that focused training be provided to committee members. Such training is often accomplished through IACUC-specific conferences and workshops, such as those offered by IACUC 101, PRIM\&R, and SCAW. In addition, several texts have been published that serve as useful references for IACUC members; these include The IACUC Handbook (Silverman et al. 2007) and the Institutional Animal Care and Use Committee Guidebook (ARENA/OLAW 2002). This training would not be as available to committee members if there was not a concomitant institutional commitment to support the activities of the IACUC. 
Box 1 Protocol elements recommended for IACUC attention in the 1996 Guide

- Rationale and purpose of proposed use of animals

- Justification for species and number of animals requested

- Availability of alternatives

- Adequacy of training/experience of personnel in procedures used

- Unusual housing and husbandry requirements

- Appropriate sedation, analgesia, anesthesia

- Unnecessary duplication

- Surgical procedures

- Humane endpoints

- Postprocedure care

- Euthanasia

- Personnel safety

The importance of institutional commitment to the proper function of the IACUC cannot be overstated. This support takes many forms, depending on the size and type of institution. For example, institutional support is often evidenced by the employment of dedicated staff, such as IACUC administrators/coordinators/directors, many of whom have demonstrated their expertise through a specialty certification program, the Certified Professional IACUC Administrator, initiated by PRIM\&R in 2006. Other methods of support [include] the provision of IACUC software products to aid in protocol and committee meeting management, as well as ensuring a direct reporting line from the IACUC to the Institutional Official, which helps to ensure timely and accurate communications between the committee and the designated responsible administrator at the institution.

\section{Changes in Protocol Review}

While the 1985 Guide made no specific mention of protocol elements that the IACUC should review, the 1996 Guide introduced several benchmarks to aid the committee in assessing the impact on the animal and to evaluate ways to foster the gathering of good scientific data while ensuring the best possible animal welfare. Items that the 1996 Guide recommended that the IACUC pay particular attention to are listed in Box 1.

The $8^{\text {th }}$ edition of the Guide (NRC 2011) added items to this list and expanded on some of the points identified in the 1996 Guide (Box 2).

Thus, over time, discussion of the subject of protocol review in the last three editions of the Guide has become more detailed and comprehensive. For example, in 1985 the Guide did not contain any recommendations specific to protocol review, in 199612 items were highlighted for attention by the IACUC, and in the 2011 version of the Guide 15 items are listed.

\section{Box 2 New and expanded-upon protocol elements described in the prepublication of the 2011 Guide}

- A clear and concise sequential description of the procedures involving animals that is easily understood by all [IACUC] members

- Impact of the proposed procedures on animals' well-being

- Postprocedural care and observation of animals

- Description and rationale for anticipated or selected endpoints

- Planning for the care of long-lived species after study completion

- Evaluation of scientific elements of the protocol as they relate to the welfare and use of animals

Also of note, the 2011 Guide has included a term not mentioned in previous editions, namely the obligation for the IACUC to conduct a harm-benefit analysis during protocol review. Specifically, the new Guide states that "the IACUC is obliged to weigh the objectives of the study against potential animal welfare concerns."

\section{The AAALAC International Perspective}

Against this backdrop of the evolution of the Guide and the concomitant increase in IACUC duties, AAALAC International has witnessed many changes in the functioning of the IACUC. AAALAC International has also offered guidance of its own in how best to fulfill the numerous responsibilities of the IACUC through newsletters ${ }^{1}$ and presentations (e.g., www.aaalac.org/resources/presentations.cfm).

AAALAC assesses the operations of the committee initially through the information provided in the program description filed by the institution with AAALAC prior to the initial and triennial on-site assessments. While on site, AAALAC International representatives review IACUC procedures and policies; protocol files and related documentation; minutes of committee meetings; the semiannual reports pertaining to the facility inspections and program reviews; and the PHS Animal Welfare Assurance, as applicable. They also tour the animal facilities and visit laboratories. In combination, this input provides a very thorough picture of the IACUC's activities and how well the committee discharges its responsibilities.

An analysis of letters sent to institutions visited by AAALAC International during 2003-2008, representing almost 1500 visits, shows a general decline in the total number of IACUC-related "findings" (Figure 1), which are categorized as "Mandatory Items" and "Suggestions for Improvement." Mandatory Items must be corrected by the institution

${ }^{1}$ See the AAALAC Connection newsletters of spring 2003, spring 2001, and summer 2000, available online at www.aaalac.org/publications/. 
Figure 1 Number of AAALAC International IACUC-related "findings" per year, 2003-2008. mandatory, mandatory corrections, SFI, suggestions for improvement

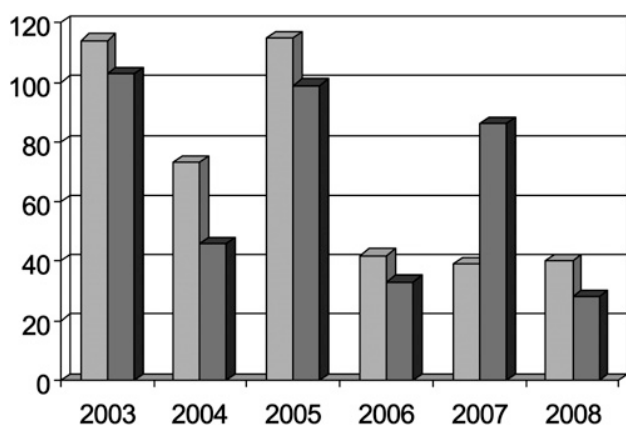

$\square$ mandatory $\square \mathrm{SFI}$

in order to be granted full accreditation; the Council on Accreditation offers Suggestions for Improvement to enhance an acceptable or even commendable animal care and use program and as a means of promoting continuous improvement in a high-quality program. The strong commitment that IACUCs at accredited institutions have made to provide appropriate oversight of animal care and use programs is evidenced by the fact that approximately $98 \%$ of institutions have Full Accreditation [from AAALAC International].

Since the publication of the 1985 Guide, AAALAC International has observed many examples of improvement in animal welfare and in the resulting quality of science as the IACUC has engaged in increasingly comprehensive oversight of the animal care and use program. Given the overarching scope of IACUC input to the animal program, the number and types of program enhancements are also broad. Examples of this improvement include

- improving the level of veterinary care provided to the animals, and a consequent minimization of pain and distress;
- $\quad$ ensuring a sound system of review of animal use proposals and of the animal program;

- $\quad$ ensuring appropriate cage space for animals;

- $\quad$ ensuring adequate sanitation and condition of cages;

- providing environmental enrichment to animals;

- referencing the Three Rs during deliberations of proposed animal work, to include an emphasis on the prevention of pain and distress as well as attention to preemptive analgesia and intensified review of humane endpoints; and

- ensuring adequate training of personnel.

This remarkable progress in fostering best practices and the Three Rs over the last 25 years is testament to the commitment of researchers, veterinarians, and their host institutions to a culture of care, conscience, and responsibility (Klein and Bayne 2007) that will facilitate high-quality science and innovation for the next 25 years.

\section{References}

ARENA [Applied Research Ethics National Association]/OLAW [Office of Laboratory Animal Welfare]. 2002. Institutional Animal Care and Use Committee Guidebook (M. Pitts, Chair, and K. Bayne, Co-Chair, Editorial Board). Available online (http://grants.nih.gov/grants/olaw/ GuideBook.pdf), accessed on March 15, 2011.

Klein HJ, Bayne KA. 2007. Establishing a culture of care, conscience, and responsibility: Addressing the improvement of scientific discovery and animal welfare through science-based performance standards. ILAR J 48:3-11.

NIH [National Institutes of Health]. 1985. Guide for the Care and Use of Laboratory Animals. NIH Publication No. 86-23. Rockville MD.

NRC [National Research Council]. 1996, 2011. Guide for the Care and Use of Laboratory Animals. Washington: National Academies Press.

Silverman J, Suckow MA, Murthy S. 2007. The IACUC Handbook, $2^{\text {nd }}$ ed. New York: CRC Press, Taylor \& Francis Group.

\section{The Future: Continuing IACUC Challenges}

\section{Jerry Collins}

s we all know, NIH is focused on improving the health of citizens of the world. That undertaking involves many activities and this symposium is focused on a key component of those activities, animal models of health and disease.

It was decided by leaders in the field, many of whom are present with us today, that performance- rather than engineering-based standards would underpin the oversight process of the use of live vertebrate animals in research, teaching, and testing in the United States. The IACUC process is an essential component of that activity and I have been asked to discuss some possible future challenges to be faced by members of institutional animal care and use committees.

Please note my use of the word continuing in the title of this presentation. In my brief comments today I will focus on familiar IACUC challenges that are likely to appear in slightly different forms in the future. 
I am grateful to my mentors over the years, many of whom are in the audience. If I get any of this right it is because of them. If I get it wrong it is because I didn't work hard enough to understand what they were trying to teach me.

\section{IACUC Responsibilities}

Before I reveal the results of my crystal ball gazing I want to remind all of us of the reality of the size of the workload imposed on IACUC members. I frequently use the following definition to express my sense of the enormity of the task faced by IACUCs.

Members of an institutional animal care and use committee are responsible for the oversight of everything that may have an adverse impact on the health and well-being of nonhuman animals and for many things that may have an adverse impact on the health and well-being of humans working with those nonhuman animals.

When we think of the semiannual program review that an IACUC must undertake it becomes immediately apparent that a continuing challenge faced by IACUCs is keeping current with many technical issues. Water quality for zebrafish; cage changing frequency for individually ventilated racks; acceptable analgesia, anesthesia, and euthanasia for reptiles; safety requirements for electrical devices used in wash rooms-these are just a few examples of those technical issues. While responsibility for complete knowledge of each topic may actually be someone else's, IACUC members must be familiar enough with issues like these to ask appropriate questions in order to determine whether their colleagues are in fact applying current standard of care practices.

Ongoing education and the development of the next generation of IACUC leaders is a challenge that we all face. We must make sure that the progress in animal care that we will hear about today and tomorrow continues to move forward with the assistance of educated, committed IACUC members.

\section{Continuing Responsibilities}

In the rush of modern society there is a tendency for ongoing processes to become rote. We must never forget that as members of an IACUC we are part of a team that is responsible for ensuring humane care for living beings. The animals described in a protocol must continue to be seen as beings that may experience pain or distress and we have a responsibility to work with the team to minimize or eliminate such adverse effects. We are responsible for the humane care of living beings!

Service on many institutional committees requires little beyond a focus on a specific charge with a specific timelinean executive committee of an institution may debate future directions of research, a departmental committee may debate future staffing. Committees like that have narrowly defined tasks and limited time lines. IACUC service is very different!
Each IACUC member must personally take ownership of his or her animal program.

Those of us involved in training IACUC members must continue to emphasize the essential need for those individuals to recognize the authority vested in the committee and the responsibility that an individual assumes when he or she agrees to serve on an IACUC. As I said, we are responsible for oversight of everything that could have a negative impact on animals.

\section{Greatest Challenge}

Although many of us have been involved in animal care/ regulations for 20-25 years it is unlikely that 20 years ago we were concerned about what it would take to provide adequate care for several thousand genetically modified zebra fish at our institution. Perhaps the greatest challenge future IACUCs face will be keeping current with what appears to be an increasing rate of change in our understanding of basic biology. Those of us currently engaged in passing on the baton have a responsibility to make sure that systems are in place to facilitate ongoing training of the next generation of self-regulators.

\section{My "Crystal Ball"}

I have chosen the following seven topics as examples of ongoing IACUC challenges: administrative support; humane endpoints; postapproval monitoring; foreign research locations; nonstandard housing; verification of training; mustshould-may. Some of them overlap, but all will require continued creativity by IACUC members to ensure humane care of animals that are essential to the goal of the NIH: improved health.

I should make it clear that although I will describe challenges, I am not foolish enough to suggest solutions. Rather, I look at this presentation as a reminder that the IACUC process is not static. It requires participation by lifelong learners. Many of the people in this room established the goals that have been approached, using performance-based standards, by many diverse routes. As science becomes more complex the number and complexity of those routes is bound to increase.

\section{Administrative Support}

Although the use of larger animals in biomedical research has been declining, the "genetic revolution" continues to drive demand for more animal facilities, which in turn requires more space, equipment, personnel, and training. Institutions continue to struggle to meet current financial needs and downsizing is a reality for many of us.

IACUCs must work with administrators to ensure that animal needs continue to be met and that pressure to expand animal use is met with reasonable increases in space, equipment, personnel, and training. It is especially important that 
programs evaluate expectations for workers directly involved in animal care. They are a unique resource and these individuals must be provided with adequate time to attend to the animals as well as the facilities and equipment.

It is also extremely important for IACUC members to know their program and, when necessary, use their authority to ensure that the resources necessary for their unique animal program are available. If, after consultation with attending veterinarians and other experts, it appears that adequate resources will not be forthcoming, the IACUC must limit aspects of the animal program that do not meet agreed-upon standards.

\section{Humane Endpoints}

Working with investigators and veterinarians to ensure that the earliest possible endpoint is chosen and implemented is, in my mind, one of the most important and challenging tasks that IACUC members face. Balancing scientific and at times regulatory need with humane concerns is not always easy. The reality of unexpected phenotypes appearing in genetically modified animals introduces an additional challenge that we did not face 20 years ago.

As is appropriate, scientists and regulatory bodies continue to refine experimental protocols and at times are able to replace animal models. National and international efforts in this arena should continue.

IACUC members must remain committed to understanding both the status of an animal associated with particular endpoints and the scientific justification for a particular endpoint. We need to continue to understand what we have approved.

Beyond that, when the chance for unexpected phenotypes is present there should be in place clear protocols for the monitoring of progeny and for the immediate reporting of potential adverse phenotypes to both the attending veterinarian (AV) and the IACUC. If adverse phenotypes are identified and a decision is made to use those animals, IACUCs should require that modifications be made to relevant protocols to explain and justify continued use of the animals in question.

\section{Postapproval Monitoring}

Postapproval monitoring (PAM) has become a cause célèbre in the IACUC community. In IACUC 101 training sessions throughout the country participants continue to ask about it. Remembering that there is no requirement for PAM per se, I do believe that it can provide an opportunity to better understand what is happening at an institution.

One pitfall that IACUCs need to avoid is the temptation on the part of some institutions to create a PAM bureaucracy without considering the underlying process. PAM is not an accumulation of personnel; rather it is a means to provide an institution with feedback on progress of animal use after IACUC approval has been granted.
As PAM programs expand, institutions must be careful to ensure that they do not replace the authority and responsibility of the IACUC. For example, since areas funded by PHS but not using USDA-covered species can be inspected by non-IACUC members, there is a temptation to "free up" IACUC members from that task by using PAM personnel for semiannual facility inspections. That should be done with caution. I firmly believe that members of an IACUC need to see, hear, and smell the animal program for which they are responsible. As I said in my opening remarks, they must take ownership. Also, if a PAM process is implemented it is important for institutions to ensure that the IACUC is informed, in a timely fashion, if significant deviations are observed by PAM personnel.

\section{Foreign Research Locations}

There are 79 foreign countries in which institutions have a PHS-approved Animal Welfare Assurance. If any of those institutions receive PHS funding in the form of a subaward from a US-based institution the expectation is that the US award recipient will be responsible for IACUC review and oversight of work conducted at the foreign location.

This problem is one that will continue to grow. AAALAC accreditation is expanding in foreign countries in response to that growth. AAALAC accreditation certainly provides an independent review of foreign facilities but it does not replace the US institutions' responsibility. IACUCs will need, in consultation with OLAW staff, to devise creative and acceptable means of monitoring foreign sites. Fortunately, communications technology is providing ever improved ways to implement real-time audio and video communications at a distance.

It is crucial for US institutions to ensure that standards approved in local protocols are met over those long distances. Fortunately, foreign institutions recognize this need and are reaching out to meet agreed-upon standards.

\section{Verification of Training}

The revised version of the Guide formalizes a trend that has been apparent for some time. Appropriate training is at the heart of the IACUC process. However, documentation of that training could, depending on how it is carried out, place yet another burden on investigators. Institutions at which documentation is in need of improvement will be best served if animal users are engaged in discussions about how to best implement this new requirement. As with so many regulatory issues, communication will be crucial.

Congress made it clear many years ago as laws were being promulgated that their intent was not to stifle research. We need to keep that in mind and individual IACUCs need to examine their processes to ensure that self-regulation is happening in ways that minimize additional workload for investigators. 
The Federal Demonstration Project recently reported on a study that indicated that a principal investigator spends about $16 \%$ of his or her time working on regulatory issues. Those numbers were not specific to animal research. Let's imagine an $\mathrm{MD}-\mathrm{PhD}$ who is caring for patients, conducting human clinical studies, and conducting preclinical work using animal models. She has to meet human investigation standards, HIPAA, and IACUC standards. If she is studying a new drug in humans FDA may be involved. If she is making genetic modifications in mice, the biological safety committee is involved. And I have not mentioned radiation safety, chemical safety, or embryonic stem cell committees. IACUCs need to be sensitive to the increasing workload associated with regulatory requirements and make sure that the committee itself does not become a source of regulatory creep.

Institutions must provide adequate resources so that IACUC staff can efficiently facilitate interactions between the committee and investigators.

\section{Nonstandard Housing}

This topic actually is a lead-in to my final point and a reiteration of a statement I made earlier. Lifelong learners are needed as IACUC members. A performance-based approach to the complex issue of humane treatment of animals used in research, teaching, and testing means that local judgment, based on sound scientific reasoning and regulatory compliance, is not only acceptable but expected.

As advances in our understanding of animal behavior and welfare are enhanced from all directions, IACUC members must be able to make informed decisions in the best interest of both the animals and the science. We are fortunate that investigators recognize the importance of service on IACUCs. It has been my experience that, although acceptance of an appointment may be done with some reluctance, once engaged in the process, thoughtful colleagues assume their responsibilities with a high level of dedication.

\section{Must-Should-May}

The performance approach requires professional input, sound judgment, and a team approach to achieve specific goals. It is essential that the desired outcomes and/or goals be clearly defined, and that appropriate performance measures are regularly monitored, in order to verify success of the process (NRC 2011, 6).

The must and should are straightforward. The may in many ways is what the scientist is all about, finding solutions to unique problems. Many people are quite comfortable with being told how to accomplish a task, they love engineering standards. Others are happier being told of a destination and then asked to devise a way to get there.

One of the joys those of us who are educators experience is the moment when the student "gets it." One of my reasons for continuing to participate in IACUC training is to experience that moment when a new IACUC member connects with the idea that she or he is in a position to maximize humane animal care, support science, and do it in a way that may be unique to the situations at his or her institution.

The future promises to provide more opportunities for IACUC members to use professional input, sound judgment, and a team approach to enhance animal welfare and facilitate scientific advances.

\section{The Future}

No system is perfect. I am certain that all of us would be quite pleased if advances in biomedical research did not rely as heavily as they do on the use of animal models. That, however, is not the present reality. We should be grateful to many of you in this room who created and molded the IACUC process as a means of local self-regulation. In the future, committed and educated IACUC members will take the process to places we can't conceive of at present.

A sign on my desk reminds me that success is a journey not a destination. The journey toward better health through the use of animal models is one that will continue to be challenging. I am confident that local self-regulation by institutional animal care and use committees will continue to contribute to better animal care, which in turn will facilitate more in-depth understanding of the biology that is at the heart of improving the health of the citizens of the world.

\section{Institutional Animal Care and Use Committees: Discussion}

Questioner: I was surprised all morning because I've heard very little about psychology so far. I am curious if there is any data on researchers' attitudes toward IACUC committees? I would think that would be really important. If people don't have a positive attitude about this procedure, it could be very difficult to get people to protect the animals they're studying. I'm just wondering if there's any data on that? Or any research?

Dr. Collins: I'm not aware of any data, but I can give you some anecdotal comments. As Dr. Dell pointed out, 25 years 
ago there was a lot of reluctance, as all of us in this room know. My experience has been that the old silverbacks like me are still somewhat challenged by the need to interact with an IACUC process. However, the younger faculty - and I'm talking specifically about those in the academic world, who have grown up understanding the process and seeing the benefit, both to the animals and therefore to the sciencerecognize it as a necessary evil. That may sound strange coming from me up here, but I think we all have to recognize, as I said in my comments, the regulatory challenges that investigators face, added to their workload. So when I say "evil," I don't mean a bad process, but rather it is an additional process that takes them away from their primary responsibility, doing science. I think that there's a balance there, a recognition of the IACUC process as something that must be done. But I do also think there is an increasing recognition that the IACUC process and the team approach to that process is something that is facilitating science as well.

Dr. Taylor: I'd also point out that it's my understanding that the majority, if not all, of the major scientific associations have committees that address the use of animals in their specific programs in their specialties. They serve as educational resources and fellow researcher guidance entities for those researchers, so that's certainly one subgroup among all the associations that could speak to relaying information about the IACUC oversight process to all those scientists.

Questioner: I have a question for Dr. Collins regarding postapproval monitoring. Do you recommend that the people doing the postapproval monitoring be IACUC members or associated with the IACUC? Or be separate personnel?

Dr. Collins: Keeping in mind, as we heard earlier, that there are as many solutions to these issues as there are issues, I encourage you to take a look at a recent ILAR publication [ILAR Journal 49:4, Animal Use Oversight: Balancing Risk Management with Burden] because there's a broad spectrum of perspectives there. My personal opinion, based on the fact that it worked very well for us at Yale, is that the postapproval monitoring personnel are an arm of the IACUC. I am uncomfortable with the idea of having a separate group of people going out and seeing what's happening out there and not having some level of direct reporting back to the IACUC. However they're associated with them, there at least needs to be that connection so that the feedback the IACUC needs is getting back to them in an efficient manner.

Questioner: My question has to do with IACUC training, not in the general sense that we're doing it now perhaps, but as a veterinarian I'll ask a veterinary question. Are veterinarians sitting on IACUCs well enough trained to be experts in all the areas you need? Surely they're not. How can that training be facilitated? Should it go back to the vet schools? Can it be postgraduate?

Dr. Collins: I'll take the beginning and then I'll let a vet or two perhaps respond to it. One of the challenges that an IACUC faces is being in a position to determine whether the current standard of veterinary care being provided at that institution is truly, in fact, the current standard of care. So you raise a very important question. I think it is very important for IACUC members to know that their veterinarians are participating in the various training opportunities that are available through the training organizations. As to how best to enhance their training, let's imagine a particular area where a weakness appears, then it seems appropriate for the institution to work with the veterinarian to make sure they have an opportunity to go to a facility where they can get that additional training.

Dr. Bayne: I would just add that I don't think any single member on an IACUC could have the broad range of background to address every single protocol coming through the institution. But collectively and through the use of consultants, that knowledge is gained. And lab animal veterinarians are a pretty small group; between CompMed and other networking opportunities, we see questions posed and addressed.... We don't know where science is going in the future, and can never anticipate fully the full range of information that a lab animal veterinarian would need to bring to the table. A solid knowledge, understanding, and skills in the basics certainly are the foundation building block.

Dr. Taylor: Just as a reminder, the PHS Policy specifically addresses the issue and addresses the use of consultants to come in and assist, aid, guide the IACUC process. So that certainly could be a method to bring in a specialty veterinarian for information that might be necessary for some of these unique animal models.

Questioner: I wonder if the panelists might comment on the outside member on the IACUC that is mandated to represent community concern for the welfare of the animal subjects? And who do you recommend? How do you recommend going out and selecting such an individual? And how you might feel about a requirement that there be two individuals, not just one?

Dr. Collins: I wouldn't agree with a requirement for two, but I would encourage all institutions to have two, not only because you can get additional input from the community but also because that's one of the people that if they suddenly are no longer available (e.g., they die suddenly) you do not have a properly constituted committee and therefore cannot carry out the responsibilities. I think that's the lesser of the two issues. I think the opportunity to have additional representation from the greater community is an important process. Certainly, over the years, in talking to many other people at many other IACUCs, it's frequently the community member who asks the question that's the most penetrating because he or she is not as familiar with everything as all the other folks are, so they're crucial.

Dr. Bayne: Part of the AAALAC assessment process is typically a lunch with the IACUC. Council members view that as a nice informal opportunity to chat with some of the key players on the committee, particularly the nonaffiliated member, and to gauge their engagement with the committee's activities. Are they really truly fully functional? Are they participating in the semis? Are they feeling intimidated? This gives the site visitors an opportunity to be informed as to how well that person is integrated into the functioning of 
the entire committee. And I will say, as I review letters, that just doesn't come up as an issue. It may be the universe of accredited institutions where the nonaffiliated member is fully functioning and feels comfortable in his or her duties. I do agree that where we occasionally stumble is when an institution has not had an alternate nonaffiliated member and finds itself lacking such a member and needing to identify one. I believe part of your question was how institutions find those individuals and what they look for? We see a variety of methods - colleagues, people at church, someone with some connection with someone on the committee or with a former member on the committee who does not have any other relationship with animal use. Qualities that most of the committees we deal with are looking for in that individual are someone who is open minded and willing to ask questions, and is not going to be fearful that because it's a rather basic question someone might think less well of them, someone [who has] that self-confidence to pose questions [and has] time, because they're just as busy as the other committee members.

Dr. Taylor: Something that I would expand on is that after 35 years of doing AAALAC site visits, I've run across a number of institutions that do have two [unaffiliated] membersat least two-on their animal care and use committees.
Perhaps some of the committees have found through the years that they are sort of echoing the IRB model (as a lot of you know, an IRB cannot do business unless there is a public member present when they're making decisions and doing deliberations). It's been my experience on site visits that by having two, as voting members, not only are you getting two outside views - especially when the investigator is not being clear, there is a requirement that the PI make [the proposed work] more clear, so that the outside member truly understands what's going on-[but also, if] one person happens to be ill or out of town, the [committee] knows it can do business and not be noncompliant.

Dr. Dell: I would just like to add a comment about the composition of the IACUC. There are a number of issues that an IACUC is faced with and therefore the IACUC must have breadth of experience. At Columbia we wound up with a committee that had twelve people on it. I think that's large for a lot of places in the country, but we felt that we needed somebody that was experienced in statistics (that was me) and somebody that was a microbiologist that could evaluate those kinds of issues - we needed a broad spectrum of expertise on the committee. We found that that was very helpful in reviewing protocols and that we could do it much more expeditiously because there was somebody there that understood. 


\section{Education and Training: Session Introduction}

\section{Mary Lou James}

T raining and education are essential ingredients for ensuring that the self-regulatory process works, [that] research animals are used only when necessary and are treated with care and compassion for decisively defensible goals. During this session, we will hear from two remarkable people whose paths to promoting education and training in the fields of laboratory animal research, albeit generations apart, have served the biomedical research community in general, and science and animal welfare in particular, extremely well.

Over my 30 years in animal research, and unlike other program components, I have often considered the successful pursuit of effective training and educational programs in animal research as one of our last frontiers-not fully explored and only partially cultivated, perhaps neck-to-neck in competition with the development of sound occupational health and safety programs.

Today, however, and after consideration of the talks you will hear during the session, I recognize that my perspective has been grossly flawed. After hearing from our presenters this afternoon, perhaps you, too, will agree that training and education are not our last frontiers but, in fact, are the most critical ones-frontiers unlike any other in our field, frontiers with no borders, that deserve our constant attention and that must reach across to our institutional officials, our IACUC members, IACUC administrative staff, veterinary and animal care personnel, research staff, personnel indirectly engaged in ensuring a sound institutional program, and, just as vital, the public.

Strong, comprehensive training programs are key to animal welfare and self-regulation. There can never be too much training. Relative to the year 1980, when I first joined the biomedical research community, tomorrow's possibilities for various training and educational forums and venues will likely boggle the mind, allowing endless opportunities for a trained and educated research community.

Our first speaker is Dr. Steele Mattingly, former Director of Laboratory Animal Medical Services and Bioethics at the University of Cincinnati. He received his veterinary degree from Auburn University in 1955. Over his many years of service to the evolution of animal care and research, Dr. Mattingly worked for Pitman-Moore, Harlan Sprague Dawley, and the Children's Hospital Research Foundation. In 1999, he was presented with the Charles A. Griffin Award, the oldest and most prestigious AALAS award for outstanding accomplishment in the improvement of care, quality, and environment of animals in biomedical research. In 2004, Dr. Mattingly received the Charles River Prize in recognition of distinguished contributions to the field of laboratory animal science. He is past president of AALAS and the American Society of Laboratory Animal Practitioners (ASLAP) and served on the AALAS, ASLAP, and ACLAM Boards of Directors. From 1969 through 1991, Dr. Mattingly organized and moderated Conmed, the Continuing Medical Education Laboratory Animal Medicine Conferences. These annual conferences were essential to veterinary continuing education in the Midwest. Today, Dr. Mattingly will speak about this early veterinary medical educational forum that paved the way to improved veterinary and animal care, animal health, and ultimately animal research. As Ralph Honaker said in response to the question "Who were your mentors?," "I feel privileged that I could learn so much from the efforts of Dr. Steele Mattingly, particularly when he held the Conmed conferences at the University of Cincinnati." I, too, am privileged to introduce this important educator who pioneered our earliest training programs.

Our second speaker is Susan Silk, Director of the Division of Policy and Education, Office of Laboratory Animal Welfare at the National Institutes of Health. Susan earned a master's in genetics from the University of Maryland and worked previously at the National Cancer Institute, Office of the Director, in many roles, including Educational Program Developer, Special Communication Project Developer, Senior Scientific Writer, and Speechwriter. She also served the NCI Intramural Program as the Senior Animal Policy Advisor and Director of the Office of Mice Advice. Susan directed transgenic mouse core laboratories at both NIH and the Johns Hopkins University School of Medicine. Today, she brings 29 years of experience in biomedical research and scientific communication to OLAW, where she oversees the development and interpretation of policy regarding research animal use and develops educational forums tailored for animal care and use programs. Over the past 4 years, I have had the honor to work with Susan as she coordinates OLAW support for several important internal and external educational programs that share the common missions of training and education. She will speak on the Role of Education in Self-Evaluation. 


\section{Conmed: The First Educational Forum}

\section{Steele Mattingly}

onmed means continued medical education. Why was Conmed started? About 50 to 60 years ago there were an awful lot of diseases in this country. A lot of people thought it was normal for the research animals to have these diseases. Let's remember some of the conditions that existed back in the 1950s and '60s. Very primitive facilities were approved by the US Department of Agriculture, and don't take that the wrong way. They did the right thing, for if they approved it, then they could tell them "to maintain this approval you will have to make these changes." If they had denied them coming in, they wouldn't have had that opportunity to make things better. Inside the building conditions were even worse than they appeared from the outside. Sanitation didn't mean anything to these people. A lot of important investigators used animals from this source.

The veterinarian at Children's Hospital approached me one day and he said, "I'd like for you to talk with Albert Sabin. I'd like for him to select animals from better places." So we had a meeting. I knew ahead of time that this was not going to be pleasant, for I had met him when I worked for Pitman-Moore Company and he came over to talk to us about making his vaccine. When we questioned him about the safety, he got mad, and we had to remind him that we were entitled to ask these questions, for in the event that it didn't work we would be sued and he wouldn't. So we knew that he had that kind of outlook on this situation. ${ }^{1}$

Why did we start Conmed in the first place? We didn't have many places that we could go for advice and counsel. Probably Notre Dame was doing more work to help eliminate diseases at that time than any other place in the country. But a lot of their work was too sophisticated to be done in the average animal facility. The rodent producers were another group that wanted to improve the quality of animals. Henry Foster did a great job of getting Charles River moving in the right direction. They knew that if they could eliminate these diseases, they would make more profits. It's the old story that the farmers knew-if you can add one more pig to the litter that's for sale, your profits go up considerably. The same thing applied to rodent production. And we knew that once we got people to start improving the animals, this would help the research community, for as they used better animals the quality of their research would go up.

How did we organize Conmed? The first 2 years of the conference were 1-day programs; we realized that probably

\footnotetext{
${ }^{1}$ If you want to know more about Albert Sabin, take time to read this bookPolio: An American Story by David M. Oshinsky—and if you're not interested in him it's still a good story about polio.
}

should be changed, and then we used the format of a day and a half. We would have eight lectures, a dinner, and then, after the dinner, a time for questions and answers. The next day we ended with four additional lectures.

We used some basic principles that I learned while I was taking graduate courses in ag education. You have to keep adults interested, and one of the ways was to have a 45-minute lecture and a 15-minute break. If the lectures are too long, many adults will go to sleep regardless of what you are saying. A 15-minute break will allow people to get up, stretch, and if they were getting sleepy, hopefully, they return awakened and ready for the next lecture. And having the participants getting together in the hall would allow them to teach each other things. They could say, "I don't agree with this" or "I do agree." When they shared their views, they became friends. This was important, for when they returned

\section{Box 1 Conmed titles 1969-1991}

Respiratory Diseases of Laboratory Animal Medicine, 1969

Selected Problems in Laboratory Animal Medicine, 1970

Automated Animal Care, 1971

Animal Resource Facility Personnel Problems, 1972

Efficiency through Planned Management, 1973

Animal Behavior, Research and Animal Housing, 1974

Educational Methods for Improving Animal Facility

Personnel, 1975

Hazard Containment in Animal Facilities, 1976

Controlling Quality of Animal Models, 1977

Regulations Governing Research Animals, 1978

Ethical Issues Related to the Use of Research Animals, 1979

Defining Laboratory Animal Diets, 1980

Strategic Management of Lab Animal Facilities, 1981

Microbial Rodent Diseases, 1982

Genetic Monitoring of Laboratory Animals, 1983

Humane Care of Animals, 1984

Laboratory Animal Facility Management Update, 1985

Updating Clinical Care Standards, 1986

Educational Programs for Research Animal Personnel, 1987

Psychological Well-Being of Animals, 1988

New Fashions for Animal Facilities, 1989

Modern Technology for the Design and Refinement of Animal Models, 1990

Laboratory Animal Facility Management, 1991 
home, they could call their newfound friends and ask more questions about how they could solve their problems.

How were the topics selected? We looked at some of the issues that were creating problems. These included changes in laws, disease control, equipment improvement, and personnel safety. All of these topics were important and people needed to know more about how to solve these difficulties to make their place more productive and also safer for all their people. Some of our titles point out how this took place (Box 1).

We started in 1969 with respiratory diseases. We felt that this was one of the leading problems at the time, and if we could find better answers to controlling it that would be a step in the right direction. Next year we put together a variety of things, followed by automated care in 1971, and then we moved to taking care of people. Then we liked to think about management and how we could be more efficient. Everybody had a budget and if you could improve your budget, you made a lot of administrators happy. And educational methods were needed to have your people properly trained, so this session was very important and useful for the people who attended. And we included hazard containment to take care of personnel safety. Back to controlling the quality of animals followed, and you had to keep up with regulations, so it was time to talk about this again. Ethical issues became a big issue at that time. That was an interesting meeting for everybody to talk about how this could be approached. Diets could not be left out, so in 1980 we spent time talking about how to improve the diets of the animals. And then back to management for the facilities in 1981. In 1982 we were back to the rodent diseases, and then in the mid-80s everybody got interested in genetics. We were aware that most of us didn't know enough about genetic monitoring, and we felt this was a good topic to have at that time. Humane care needed to be stressed again, and then we went back to management and clinical care in 1986. In 1987, we had educational programs for the personnel again. (You never have everybody completely up to date and we had a variety of people attending. Not everyone attended every year so we felt we could have some duplication without any difficulty.) Psychological well-being was a topic that had to be addressed in 1988. New fashions for running your facility gave a different way to look at how to construct a facility. And design and refinement of animal models followed. And the last year, we looked at laboratory animal facility management.

Someone always asks, "How did you select the 12 topics for that day and a half?" Well, I called someone and said, "This looks like a good topic that should be discussed today. How would you break it down?" I was not afraid to ask for help and call friends and get their advice and counsel to come up with these topics. They were always willing to help.

Some of the examples will be in this 1991 program (Box 2). We had this broken into parts: (1) improving the institution's public image, (2) improving the public's perception of a career in laboratory animal medicine, and (3) the institutional administrator's role in education of the public. I was not afraid to ask people that were doing important things, like Charlie McCarthy, to help and be a speaker at the meeting. He did a great job, presenting a positive and truthful story about research animals. And there was a lecture on building an animal research program after negative publicity. We knew we were going to have some negative publicity. The local humane groups knew we would have this meeting the last week in April, so they would be ready to picket and have their signs out like "Mad Dog Mattingly" and all those kinds of things. When we had that negative publicity, we needed to know more about how to manage it. Then the afternoon series of lectures was monitoring the pathogen level of animal colonies, looking at disease control prevention (Tom Wolfle is here today and I'm sure he remembers some of the things he shared with us at that time). The last of that session, before dinner, was on pain control. The next day we had a session on determining the total cost of operating an animal facility, determining who should pay for various parts of a budget, management during a

\section{Box 2 Topics and speakers for the 1991 program}

Improving the Institution's Public Image

- Improvement of the Public Perception of a Career in Laboratory Animal Medicine - David Moore, DVM

- The Institutional Administrator's Role in Education of the Public - Charles McCarthy, PhD

- Presenting a Positive and Truthful Story about Research Animals - Sandra Prell, MBA

- Building an Animal Resources Program Following Negative Publicity - Harry Rozmiarek, DVM, PhD

Improvement of Creature Comforts

- Monitoring the Pathogen Level of Animal Colonies - Joseph E. Wagner, DVM, PhD

- Disease Control - Prevention in Rodent Colonies - Robert J. Russell, DVM

- Creature Comfort? Just Ask the Animals! - Thomas Wolfle, DVM, PhD

- Pain Control - Gerald Gebhart, PhD

Budget Management

- Determining the Total Cost of Operating an Animal Facility - Farol Tomson, DVM

- Determining Who Should Pay for Various Parts of the Budget - Bruce H. Ewald, DVM

- Management During a Recession - Dale L. McGirr

- The Impact of Federal Regulations upon the Institution's Budget - Nelson L. Garnett, DVM 
recession, and the impact of federal regulations on the institution's budget. Don't forget that the discussions after the first day's eight lectures were important, for we got a lot of good information from everybody in the audience at that time.

When we started, we charged $\$ 30$ for attending; when we ended, the price had moved up to $\$ 150$. That included breakfast, two lunches, and a dinner.
Today, if I had to make some changes in this, I'd take my son's advice. He's an adjunct professor at a law school, and he says your lectures should be broken into 10-minute topics. I think this is true, and I would ask the speakers to take that in consideration. The American Society for Training and Development has developed guidelines for training, and I think more of us should know what they're doing and how they could help us out.

\section{The Role of Education in Self-Evaluation}

\section{Susan Silk}

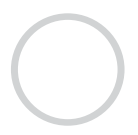
ne of the great pleasures of my job at OLAW is supporting educational programs. I think the most important aspect of our system of animal welfare oversight in the United States is education. Why? Because our system is based on self-evaluation.

There have been times in our history when self-evaluation was called self-regulation or even enforced self-regulation. Whatever we call it, institutional animal care and use committees are central to this system that relies heavily on professional judgment and performance standards. Performance standards, of course, are the system in which individualsIACUC members, veterinarians, or scientists - draw upon their education and experience to determine the best course of action in a specific situation. Engineering standards, at the other end of the continuum, define each step to be taken to achieve a desired result.

Biomedical research is complex and ever changing. Our animal welfare oversight system is highly flexible and selfcorrecting because the system is based on the judgment of highly trained, well-educated professionals.

The idea of self-evaluation came from the $90^{\text {th }}$ Congress of the United States, which passed the Health Research Extension Act of 1985. This is the statute that gives OLAW the authority to administer the PHS Policy. The Congressional Report that accompanied the Health Research Extension Act said, "It is far preferable to place primary responsibility for assuring compliance with the NIH guidelines on committees within institutions, rather than relying on intrusive federal inspections." They were, of course, talking about IACUCs.

Although grantee institutions, scientists, IACUC members, and animal program personnel are expected to exercise their best professional judgment, they must do so within the framework of a multitude of rules and regulationsnot only federal regulations but also the requirements of their state and local jurisdictions and even of their institutions. Where do all these regulations come from? At the federal level, there are a lot of agencies involved in animal welfare oversight, as you can see in this list of federal animal welfare regulators, whose roles and responsibilities I'll summarize:

- NIH Office of Laboratory Animal Welfare (OLAW)

- USDA Animal Care

- USDA National Agriculture Library, Animal Welfare Information Center (AWIC)

- Centers for Disease Control and Prevention (CDC)

- Food and Drug Administration (FDA)

- US Customs

- US Fish and Wildlife Service

- National Marine Fisheries Service

At OLAW, of course, we oversee the welfare of research animal subjects in PHS-funded activities.

The Animal Care program of the USDA Animal and Plant Health Inspection Service regulates the use of most warm-blooded animals in research and exposition and includes vendors. AWIC is another USDA group, part of the National Agricultural Library. AWIC conducted a preconference workshop, Meeting the Information Requirements of the Animal Welfare Act, yesterday. Although sponsoring the workshop was a special honor for OLAW, it was business as usual for the great folks at AWIC. They conduct this workshop numerous times throughout the year to fulfill their mandate, which is stated in the Animal Welfare Act: "To provide information for the improved care of animals used in research, testing, training, and exhibition." The workshop provides training in how to find the latest refinements in research techniques and alternatives to animal use.

CDC oversees the importation of nonhuman primates and provides the manual Biosafety in Biological and Microbiological Laboratories (BMBL). The FDA oversees good laboratory practices (GLP) for our partners in industry. The US Customs Service makes sure animals are imported under humane conditions according to the Lacey Act. The US Fish 
and Wildlife Service is responsible for the import, export, and research use of certain wild marine mammals. And certain marine mammals enjoy the protection of the National Marine Fisheries Service.

How can well-intentioned individuals and institutions obey all of these regulations? A good animal care and use program is made up of individuals who are continually increasing their knowledge through education, training, and experience. Everyone in the program must be engaged. How do we get all of these people working toward a common goal? Education. The team changes constantly, so there is a continual need for education, as we all learn and grow throughout our careers. IACUCs, especially, experience a constant turnover of members in these unpaid committees that require so much time and work from their members.

The government agencies with overlapping responsibilities for animal welfare oversight also have educational components. But we don't do the heavy lifting alone. Steele [Mattingly]'s outstanding program [Conmed] was the first, and it inspired many more outstanding programs. I hope I will not steal Dr. McCarthy's presentation if I say that these later programs are standing on the shoulders of a giant.

Most of the educational organizations are represented in the audience today and many have materials displayed in the hall. IACUC 101 has been training people with basic and advanced workshops since March 1998. They held a preconference workshop here yesterday. Mary Lou [James], who keeps a very accurate tally, tells me that as of yesterday they have trained 9717 people.

Public Responsibility in Medicine and Research (PRIM\&R) started providing IACUC training in 1997. I cannot even guess how many people have attended PRIM\&R's Annual IACUC Conference over the years. Many of us are at work now developing the 2011 Conference.

Scientists Center for Animal Welfare (SCAW) conducts workshops all around the country. They were in Bethesda last month and in Chicago last week. Many of us will be at the SCAW Annual Winter Conference in Texas in December.

CITI, the Collaborative Institutional Training Initiative, provides online training. Their menu of training continues to grow through the work of dedicated volunteer developers, many of whom I see in the audience today.

A new grassroots organization that empowers IACUC administrators to network together and develop best practices is the IACUC Administrators Association, which conducts the IACUC Administrators Best Practice Meetings.

Certified Professional IACUC Administrator (CPIA), another grassroots initiative, enables IACUC administrators to receive professional certification credentials.

AAALAC plays a unique role as a voluntary accrediting agency. It provides education not by telling an institution what to do but by being an extra set of eyes that provides an assessment of an animal program by experts who are not affiliated with the program.
AALAS has been doing it all and for a very long time. The annual AALAS meeting trains thousands each year. They offer online, print, and in-person education. They developed and administrate the LAT (laboratory animal technician), ALAT (assistant LAT), LATg (laboratory animal technologist), and CMAR (certified manager of animal resources) training and certification.

The Laboratory Animal Management Association (LAMA), Laboratory Animal Welfare Training Exchange (LAWTE), ASLAP, ACLAM, AVMA, American Association of Veterinary Medical Colleges (AAVMC), ILAR, and Lab Animal magazine - these organizations train and educate the people that provide clinical care and husbandry and run increasingly sophisticated animal facilities.

Thank you to all of you.

Here are a few of the many professional organizations that provide their members with written and online information about research animal subjects and animal models: Society for Neuroscience (SfN), American Physiological Society (APS), Foundation of American Societies for Experimental Biology (FASEB), and American Association for the Advancement of Science (AAAS).

And here is a representative sample of organizations and individuals that work to educate the public: Americans for Medical Progress (AMP), Foundation for Biomedical Research (FBR), National Association for Biomedical Research (NABR), Incurably Ill for Animal Research (iiFAR), and the many state organizations that function in their individual locations and are joined together under States United for Biomedical Research (SUBR).

ProTest and Speaking of Research are organizations that explain the value of biomedical research to the public. Michael Conn and James Parker's book, The Animal Research War, is representative of the many authors who write editorials, articles, and books that explain the benefits to human and animal health derived from biomedical research.

Now I am going to try to look into the crystal ball. In the future, I think we will see increasingly sophisticated online offerings that will be targeted to more and more specific audiences. OLAW entered this arena recently with our online seminar series and we are planning to grow with the technology.

I've heard that page numbers were not used in printed material until about 70 years after the invention of the printing press. I don't know if this is true or an allegory, but I do think we will have to grow into all the new educational technology and use our best creative and professional judgment to take maximum advantage of these new opportunities.

I am certain there will always be a need to come together in the same room and learn as we are doing here. I am especially happy because we have three professional generations here in the audience - we are able to learn from mentors and heroes and also get to know the next generation, who I hope will organize the $50^{\text {th }}$ anniversary symposium. 


\section{Education and Training: Discussion}

Questioner: I don't have a question, but I do have a comment and it's actually a thank you that goes right back to OLAW for the webinar series that you are conducting. I think this is a great opportunity for OLAW to reach out into the community. I really appreciate those training sessions and having the opportunity to review them occasionally. We aren't able to attend when they are first scheduled so to have the opportunity to look at them later at our own convenience is a real plus.

Mrs. Silk: I appreciate your feedback. We also love those online seminars. We had one participant send us an email picture of flowers, so I think we got flowers for the seminars. As the technology improves and we become better at managing it, we do intend to expand the program. We like the fact that we have access to a lot of you and it only takes an hour of your time and it's so much less expensive-and nobody has to climb on an airplane. It'll never replace face-to-face education, but it's a wonderful supplement, I think.

Ms. James: I'd like to ask the audience to give us your feedback as far as what you want for education and training, either in your own institutions or at public forums such as this. I find the webinar series are tremendous for me. I am on the road all the time; I'm rarely in the office to sit down and watch a webinar. However, OLAW has been making those webinars available. It's wonderful to have that available. It's a lot less expensive and certainly there is no excuse, any longer, for institutions not getting their individuals trained.

Mrs. Silk: Our next one is December $10^{\text {th }}$.

Questioner: Speaking of webinars, what about...the Animal Welfare Information Center's helping PIs do searchessomething interactive, a webinar? Something where they could throw in their key words, their animal model, and AWIC could demonstrate online how to do it. I think that would be very helpful.

Mrs. Silk: I know there are some AWIC people here today.

Questioner: I think that over the years the IACUCs have been doing a form of harm-benefit analysis when they look at protocols. And I do appreciate that that is outlined more in the new Guide, but I still think that we are sometimes subject to criticism about how we do that. So I think that having some type of training, hopefully an interactive type of training workshop, might help us to meet those criticisms even though I think we're doing a good job already.

Mrs. Silk: We have an online seminar about that. You can find it on the Web.

Questioner: Dr. Mattingly, we have heard today and we know that there was significant resistance to the rules and regulations of 1985 among the research community. What happened among the veterinary community? What was the reaction you saw as Conmed moved forward and as the regulations developed and were implemented?

Dr. Mattingly: I think a lot of the people felt like they knew what they were doing [and that] these were outsiders coming in and trying to tell them how to do things. They were well prepared, the outsiders, in the matter of reeducating these people and making sure they understood that some of these new regulations would eventually be of great benefit to them.

Questioner: I think OLAW and the government agencies do an excellent job of educating the staff and the committee members. One area that is, perhaps, lacking is the nonaffiliated committee members. They often have to figure out what their role is, what their duty is, and whether they are responsible for knowing all the scientific aspects of the protocol. What do they do? That is one area where I think we could do a better job.

Ms. James: Several years ago, I believe this was an OLAW-sponsored course (OPRR back then), we did have a conference, I believe in Washington DC, that targeted the nonaffiliated member. I agree, it's time we did something again for nonaffiliated members. We have community members now that have been on IACUCs for years and years. These are the best people to educate. Maybe we can get some support to put together a program like that.

Questioner: As we have an increasingly diverse workforce and as we become more global—with 79 countries having a PHS Assurance-has there been consideration of putting this education in different languages? I know AALAS was reluctant to test in another language. I'm not sure where they are with that right now, but that's a concern where I am. I teach people for whom English is a second language.

Mrs. Silk: I know the CITI modules are in several languages and they have an international reach.

Dr. Mattingly: I think when you look at the workforce, they're coming to us from a background of their formal education and we need to be sure that the institutions that offer a 2-year associate's degree or a baccalaureate degree are providing the proper information in the curriculum. It's up to us to make complaints when we feel it's necessary.

Questioner: A follow-up to the questions about training community members. The Massachusetts Society for Medical Research (MSMR) has developed a training program for IACUC community members and has actually succeeded with the number of people we can run in that program at any given time. Just another way of looking at ways to train community members on the committee.

Ms. James: How is that available to people that are not members of MSMR?

Questioner: You do not have to be a member of MSMR. 


\section{Veterinary Care: Session Introduction}

\section{Patricia Brown}

ur topic for this next panel is veterinary care. Like all the other sessions today, we'll focus on where we were, where we are today, and where we're going. For veterinary care and the role of the veterinarian, there has been considerable change in the last 25 years. We have four speakers who will address this topic: (1) from the 1985 perspective, (2) how we care for animals today, (3) the new technology that we have available, and (4) how we can learn more about our animals - in particular animal cognitionthat would allow us to improve animal welfare.

Our first speaker is Dr. Gerald Van Hoosier, professor emeritus of comparative medicine at the School of Medicine at the University of Washington in Seattle. He obtained his veterinary degree from Texas A\&M University and then focused his career on the development and safety evaluation of poliomyelitis vaccine. He served as a commissioned officer in the US Public Health Service at the National Institutes of Health in Bethesda from 1957 to 1962 . He was on the faculty at Baylor College of Medicine and at Washington State University before joining the University of Washington. He played a key role in the development of tutorials on laboratory animal medicine in collaboration with the American College of Laboratory Animal Medicine (ACLAM). He has served as president of ACLAM and AALAS and has been involved as a member of the governing board of ICLAS. He is responsible for approximately 100 scientific papers and was coeditor of Laboratory Hamsters, one of the series of texts by ACLAM, and Laboratory Animal Science, volumes 1, 2, and 3, published by the CRC Press. He served as editor of the journal Laboratory Animal Science from 1995 to 1999. He is the recipient of the AALAS Griffin Award and the Nathan R. Brewer Lifetime Achievement Award from ACLAM.

Our second speaker is Dr. Michael Talcott, Director of Veterinary Surgical Services in the Division of Comparative Medicine and a research assistant professor of Surgery and Neurosurgery at Washington University School of Medicine in St. Louis. Dr. Talcott received his veterinary degree from Michigan State University, was an NIH postdoctoral fellow at Wake Forest University Bowman Gray School of Medicine, and completed his postdoctoral training in laboratory animal medicine at the University of Alabama at Birmingham School of Medicine and Dentistry. He is an ACLAM Diplomate. His team provides investigators who use dogs, cats, swine, sheep, goats, rabbits, and other nonrodent animals with services for minimally invasive surgery, orthopedics, vascular surgery, transplantation, cardiology, neurosurgery, and general surgery. He's involved in many efforts to educate the public about the use of animals in research, including presentations for students in local schools and tours for local students in elementary school through community college. He's a regular presenter for Washington University's MiniMed School Program and for the Mid-Continent Association for Agriculture, Biomedical Research and Education (MAABRE).

[Our third speaker will be] Dr. William White. Dr. White was my mentor when I did my lab animal residency at the Hershey Medical Center in the early 1980s. He received his veterinary degree from the University of Pennsylvania and a master of science in laboratory animal medicine from the Penn State University. He is a Diplomate of ACLAM and also of the European College of Laboratory Animal Medicine. Before joining Charles River, Dr. White was a tenured associate professor of comparative medicine at the Milton S. Hershey Medical Center, where he conducted basic research in the effects of environmental variables on laboratory animals and laboratory animal anesthesia. He joined Charles River in 1988 and is currently Vice President for Veterinary and Professional Services. In this capacity he oversees the corporation's worldwide diagnostic and professional services activities as well as its corporate biosecurity program. He has had an ongoing interest in the welfare of laboratory animals and in optimizing the environmental conditions under which they are maintained. He's a member of the International Air Transport Association's Live Animals and Perishables Board's Animal Care Team. He has authored or coauthored 75 peer-reviewed research articles, and served on the ILAR committees that developed the 1996 Laboratory Animal Management Guide for Rodents and the 1996 Guide for the Care and Use of Laboratory Animals. He coedited the ACLAM text on Anesthesia and Analgesia in Laboratory Animals and has been a member of the editorial board of the Journal of Comparative Medicine.

The last speaker of our panel is Dr. Brian Hare, an assistant professor of evolutionary anthropology at Duke University. He studies cognition of domestic dogs at the Canine Cognition Center and is also involved with the Hominoid Research Group, comparing the psychology of hominoids with both human and nonhuman apes, seeking to identify which features or social problem-solving abilities have evolved since humans, chimpanzees, and bonobos shared a common ancestor. His interest in psychology has now expanded into an interest in animal welfare and we thought it was really pertinent to have him talk about his research in the veterinary care session. 


\section{Veterinary Care: Where We Were}

\section{Gerald Van Hoosier}

$\mathrm{s}$ a preface to my presentation, I want to express how much I am indebted to NIH for the beginning of my career after graduation from veterinary school. I feel like being on the campus here really imprinted on my DNA an appreciation for research and the use of animals in research for a very worthy purpose - the evaluation of poliomyelitis vaccine, and my first scientific publication (van Hoosier et al. 1961). I also want to express appreciation for the support that NIH has given over the years to various animal resource programs, various comparative medicine training programs, the primate centers, and various grants for research in the laboratory animal field that helped us to understand more about the diseases of laboratory animals that complicated their use in biomedical research.

An alternative title for my presentation could be "Flashback." My assignment is to go back in time approximately 25 or 30 years. There have been a lot of changes since 1985, so one should not assume that the way it was done back then is the way it's being done today.

\section{Challenges in 1985}

The concerns we had at that time included surgery, analgesia, and intercurrent infections. For many animals barbiturates were the primary anesthetic available. Other chemicals included chloral hydrate in rats for anesthesia and ether for anesthesia in mice, which created other problems in the laboratory, and infections in laboratory animals, some of which were transmissible to humans.

At the first meeting of the committee that revised the Guide in 1985, Steve Pakes, who chaired the committee, shared with us a conversation he had with Al Jonas, who was from Yale. Al had participated in an AAALAC site visit at a veterinary school and was appalled at the course description and practices for teaching surgery in veterinary schools back in that era. The veterinary student surgery class used dogs and performed multiple procedures, including skin incisions with different closures, abdominal explorations, intestinal anastomoses, enucleations, and there could have been other procedures before euthanasia, depending on the circumstances and the course description. The PHS Policy states that exceptions should not be made for purposes of teaching or demonstration.

The committee was considering allowing only one major surgical procedure on an animal if the procedure involved an unrelated project. In association with this proposal, one challenge was establishing criteria for the classification of surgery as major or minor. Before drafting the text for this section, I went to our university hospital and met with several surgeons to learn what they did on an outpatient basis as opposed to surgery requiring overnight accommodations. I tried to extrapolate from that to what we might classify as minor procedures. The committee edited and approved the section-the first statement on the subject to appear in the Guide.

Regarding analgesia for the alleviation of pain, only two or three drugs were available, depending on the species. We had opioids and nonsteroidal anti-inflammatory drugs (NSAIDs; e.g., aspirin). Not much was known about potential adverse effects complicating the use of these analgesicsdecreased response of the respiratory center to $\mathrm{CO}_{2}$, prolonged bleeding time and contraindications in pregnant animals, and the narrow safety margin. Back then, and also today, the complicating effects to experimental procedures sometimes limit the use of analgesics. One example is in experimental cancer research-certain analgesics inhibit the growth of tumors. There are also issues with research involving strokes in rats where the cerebral artery is occluded; some analgesics have been shown to alter the development of ischemic lesions.

Another major challenge and issue at the time we were revising the Guide was intercurrent infections in laboratory animals. One specific example was upper respiratory infections of dogs from random sources. There were also problems with zoonotic infections, and a good example involved hamsters used in cancer research: transplanted tumors became infected with lymphocytic choriomeningitis virus, which spread to tumor-free animals in the colony. Not only did this cause infections of the technicians and faculty working in the laboratory, but hamsters got into the private trade of pet animals and the infections began to occur in people who were buying hamsters as pets.

Other challenges and areas of concern were death as an endpoint, problems associated with genetically altered animals, and the source of dogs and cats (whether they were random source or purpose-bred animals).

\section{The Guide in 1985, 1996, and 2011}

The general outlines of the major topics [in the Guide over the years] are similar in some areas such as anesthesia and analgesia and preventive medicine, although surgery, diagnosis, 
and treatment of controlled disease are now under preventive medicine in the 2011 Guide. It is noteworthy that there are a couple of new areas that we didn't see back in 1985-the assessment of animal well-being and the management of pain and distress.

Is it time to redefine major operative procedures because an animal that has experienced one penetration of a bodily cavity cannot be used for another study involving such procedures? The question was recently addressed in an editorial in JAALAS by Yates and Toth (2010). An unintended side effect of this policy is that overly conservative classifications of procedures as major surgeries can require the use of greater numbers of animals for research, ${ }^{1}$ which is in conflict with the Three Rs for reducing the number of animals used in research.

\section{Looking Back}

There are issues that became more or less pivotal over time, and training materials and trainers are important facets of the changes. We currently require training not only for faculty and their technicians but also for undergraduate, graduate, and postdoctoral students.

Another major change is the increased use of analgesia, although we still grapple with questions about the extent that they complicate an experimental procedure. New technologies such as endoscopic surgery and how they're classified have been a major change. More complete review and monitoring of animal care and use, compared to what we had in 1985, have resulted in the need for a large increase in staffing of the IACUC to comply with the Guide, AAALAC, and PHS Policy.

Biomedical research using dogs was not a problem we addressed in the 1985 revision of the Guide and their use has been a major contributor to advances in medical progress over the years. Foremost among these goes back to 1923 with the use of dogs in the discovery of insulin and its use in

${ }^{1}$ As an example, in the recent past laparoscopic surgery to collect eggs from nonhuman primates was classified as major surgery. the control of diabetes. ${ }^{2}$ Another example of contributions is the Nobel Prize in 1990 awarded to Donnall Thomas and his coworkers at the University of Washington and the Fred Hutchinson Cancer Research Center for their extensive studies in dogs on bone marrow transplantation, which is now applied to humans for the treatment of various types of malignancies.

Looking back on where we were, the top three things to know about the past are that (1) there were multiple procedures being done in surgical classes, (2) there was a limited number of drugs to use for analgesia, and (3) laboratory animal medicine has evolved in the context of human medicine.

I remember talking to a surgeon back in the 1970s who didn't want to use analgesia in humans and thought it was best to just get them up after surgery and get them moving so that they didn't develop too many adhesions or other problems complicating recovery. Both laboratory animal medicine and human medicine have evolved over the years.

\section{Acknowledgments}

I thank Steven Pakes and Charles McPherson for their review of the PowerPoint program and Denise Newsom for technical assistance with the PowerPoint images.

\section{References}

Van Hoosier GL Jr, Kirschstein R, Abinanti FR, Hottle GA, Barron S. 1961. The safety test for poliomyelitis vaccine. I. An evaluation of variable factors in the monkey and cell culture system. Am J Hyg 74:209-219.

Yates B, Toth L. 2010. Is it time to redefine "major operative procedures?" JAALAS 49:8.

\footnotetext{
${ }^{2}$ There is an exhibit at the New York Historical Society, "Breakthrough: The Dramatic Story of the Discovery of Insulin," that tells the story of one extraordinary New York girl, Elizabeth Evans Hughes, daughter of the statesman and jurist Charles Evans Hughes, who was one of the very first patients to be saved by the use of insulin.
}

\section{Current Standard of Care and Technologies: Nonrodents}

\section{Michael Talcott}

' $m$ honored to be speaking with my colleagues about the improvements in animal care over the last 25 years, especially with regard to nonrodent or what I'll refer to as "large" animals. As I looked back to the 1980s I found that I had a unique perspective because I had just begun my career in laboratory animal medicine in the 1980s. So having grown up with the 1985 Guide, many of the changes that Dr. Van Hoosier related were things that I saw as the norm going through my career.

I started my career at an institution that was very typical of most major medical schools where Class B dogs were the 
predominant large animal model. They were used in the areas of cardiology, transplantation, and general surgery, and were often used for medical school training. Often, these animals required additional conditioning and treatment for common parasitic diseases. Some were euthanized because they were unsuitable for research-they were heartworm positive or had some other condition. But in the late 1980s and early 1990s the number of dogs used dropped dramatically from an average of about 186,000 in 1985 to an average of about 70,000 today (Figure 1). According to the 2009 NRC report Scientific and Humane Issues in the Use of Random Source Dogs and Cats, this reduction was multifactorial, caused by reduced access to random source dogs, reduced research funding, changing NIH program priorities, regulatory changes, and increased availability of alternative animal models among other things.

Twenty-five years ago a large number of animals were used for medical training, both human and veterinary, because there were no alternatives. Today, large-animal use for medical and surgical training, and in particular the use of dogs, has been reduced with advances in computer simulators and inanimate models. With the advent of the 1985 regulations, the research community was obliged to look for and develop alternatives to using animals and now very sophisticated inanimate and animate computer simulation trainers are available. These alternatives help to develop basic skills like suturing and vascular access and have reduced the overall number of animals used for training procedures. These systems can be programmed to create specific scenarios to help students think critically and troubleshoot specific medical diseases and situations.

However, while these inanimate models are a means to reduce the number of animals used for teaching, they do not necessarily replace all live-animal training. Inanimate models allow for practicing basic skills and critically thinking through situations, but there are many situations where live tissue is necessary to adequately hone the techniques necessary to treat a disease or save a life, human or animal.

Other influences accounted for the reduction in dog use in the 1980s, including the introduction of transgenic rodent models as a powerful research tool. In addition, more agricultural large animals were used based on their unique characteristics. Pigs were used for cardiovascular research because of their susceptibility to atherosclerosis and coronary artery disease, general surgery because of the similar size of organs (kidney, liver, stomach, and intestine), and development of surgical devices. Sheep and goats were used for cardiopulmonary research due to the large chest cavity, orthopedic procedures because of the size and maturity of the bones, and fetal surgery because of access to the large size and small number of fetuses.

Rabbits weren't often seen as the favored nonrodent species because of the pervasiveness of pasteurellosis in many rabbit colonies. This organism accounted for numerous disease states that could negate research results and lead to high morbidity and mortalities during surgical procedures. With advances in surveillance, diagnostics, and "proper" treatments, as outlined by the 1985 regulations, most of the

Figure 1 Use of dogs and cats in research

$$
\begin{gathered}
\text { Numbers of Dogs \& Cats Used in Research, Teaching } \\
\text { and Testing, 1985-2007 }
\end{gathered}
$$

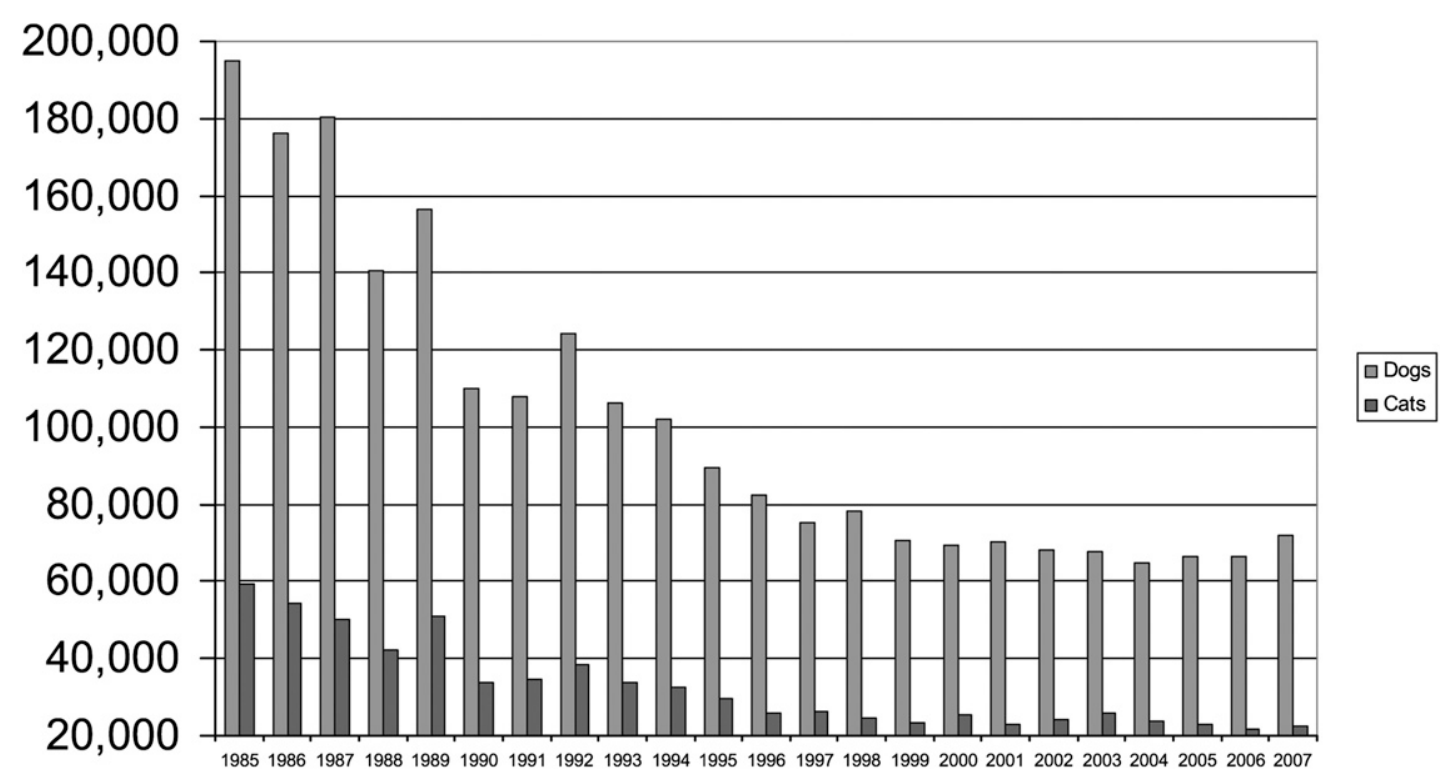


research rabbit colonies are now pasteurella-free and rabbits are used for many complex surgical procedures.

Advances in surveillance and diagnosis of diseases have also contributed to healthier agricultural models, and many diseases seen years ago — such as atrophic rhinitis and pneumonia (Haemophilus/Mycoplasma) in pigs, sore mouth (orf), pinkeye (Chlamydia), and diarrhea (C. perfringens) in sheep and goats-are seen less often, if at all, in research colonies now.

The 1985 regulations also required appropriate use of tranquilizers, analgesics, and anesthetics and appropriate pre- and postsurgical care of animals used for research. Twenty-five years ago, sodium pentobarbital and Harvard ventilators were still used for some procedures and often rudimentary methods of monitoring physiologic parameters were used. Monitoring was frequently based on the type of research. For instance, cardiovascular studies would monitor invasive blood pressure, ECGs, and electrolyte concentrations but for an orthopedic procedure, a technician may have monitored only heart and respiratory rates using a stethoscope. "Advanced technology" was seen as using halothane and audioesophageal stethoscopes.

Today the standard of care in clinical veterinary medicine and in the research community is the use of multiparameter monitoring systems that monitor pulse oximetry, capnography, NIBP and IBP, ECG, body temperature, heart rate, and respiratory rate. Short-acting and safer volatile anesthetic gases such as isoflurane and sevoflurane have replaced methoxyflurane and halothane, and state-of-the-art operating rooms are used for procedures.

Preoperative health assessments and planning between veterinarians and PIs now help to identify proper anesthetic regimes, analgesics, monitoring equipment needed for the procedure, complications that could occur with the procedure, and anticipated postoperative care.

Twenty-five years ago, postoperative care usually meant putting an animal in a cage with a heat lamp and checking on it in the morning. Now sophisticated ICU units are used to prevent hypothermia and the animal's postop status is closely monitored. Postoperative care often includes behavioral assessments or a pain scoring system to assess the need for analgesics or other postoperative treatment (such as protective bandages, soft bedding), and medical records are maintained to adequately communicate the animal's condition with research staff.

Pain relief used to be administered after recovery from anesthesia or only when pain was identified and usually consisted of short-acting opioids (nalbuphine, morphine) or longer-acting NSAIDS (banamine, phenylbutazone). Today, the use of preemptive analgesics to prevent the animal from experiencing pain or preventing more extensive pain (wind-up) is more common. Longer-acting agents such as buprenorphine, tramadol, COX-2 inhibitors (carprofen, meloxicam, previcox), and fentanyl transdermal patches are used postoperatively as primary analgesics or oftentimes in a multimodal combination to prevent breakthrough pain. Newer drugs that are specific for neuronal pain, such as gabapentin, methods to deliver drugs to the exact location of the pain using seep catheters, and analgesic effects of older drugs like ketamine and other NMDA inhibitors are being investigated to provide pain relief for the future.

Animal behavioral welfare has also changed dramatically over the last 25 years and methods used to address the social needs of an animal have rapidly evolved.

Although the emphasis 25 years ago was on environmental enrichment for primates and group housing compatible dogs, the concept of behavioral welfare is now applied to multitudes of species including cats, ferrets, rabbits, agricultural animals, and, of course, primates and dogs. Caging systems have been designed for social housing, and when possible group housing in larger enclosures is seen more frequently for certain animals. Opportunities for exercise and positive human interaction are also being used more often, with exercise rooms and time scheduled to interact with animals (Figure 2).

But while the concept of enriching animal environments and addressing social needs is being embraced by the research community, what's unknown is how these environments and social interactions may alter scientific outcomes. Although it seems inherent that group housing of animals is beneficial, there are unforeseen stresses such as competition for food, establishing hierarchical dominance, and transmission of diseases that could affect research results and animal well-being. So there's still information needed and future studies should be considered to address how these enhancements may influence scientific outcomes.

A major focus using nonrodent animals over the past 25 years has been the development of specialized surgical procedures, with an emphasis on becoming less invasive, minimizing postoperative pain, and enabling a faster recovery. Minimally invasive surgery blossomed in the 1980s and '90s and laparoscopic procedures were developed for every facet of surgery using rabbits, sheep, dogs, and in particular pigs.

As mentioned earlier, pigs' organs are very similar in size and structure to humans and so pigs have become the primary laparoscopic model. They have been used for surgical device development and have helped to establish standard procedures for laparoscopic cholecystectomies, ovariohysterectomies, intestinal resections, and hernia repair, among others. Kidney-sparing urological procedures for cancer treatment, such as cryoablation techniques and partial nephrectomies, were developed using pigs.

While these minimally invasive procedures were developed for human application, they cross medical professions easily and are used more and more frequently in both veterinary clinical and research applications. A paper published in JAVMA in 2005 showed that dogs spayed laparoscopically experienced significantly less pain than those that were spayed conventionally, and more veterinary schools are training in laparoscopic procedures. And it's appropriate that research patients also benefit from this advanced technology, so minimally invasive procedures are often used as a refinement in technique compared to "open" 


\section{Figure 2 Laboratory animal enrichment: Op- portunities for exercise and for interaction with conspecifics}
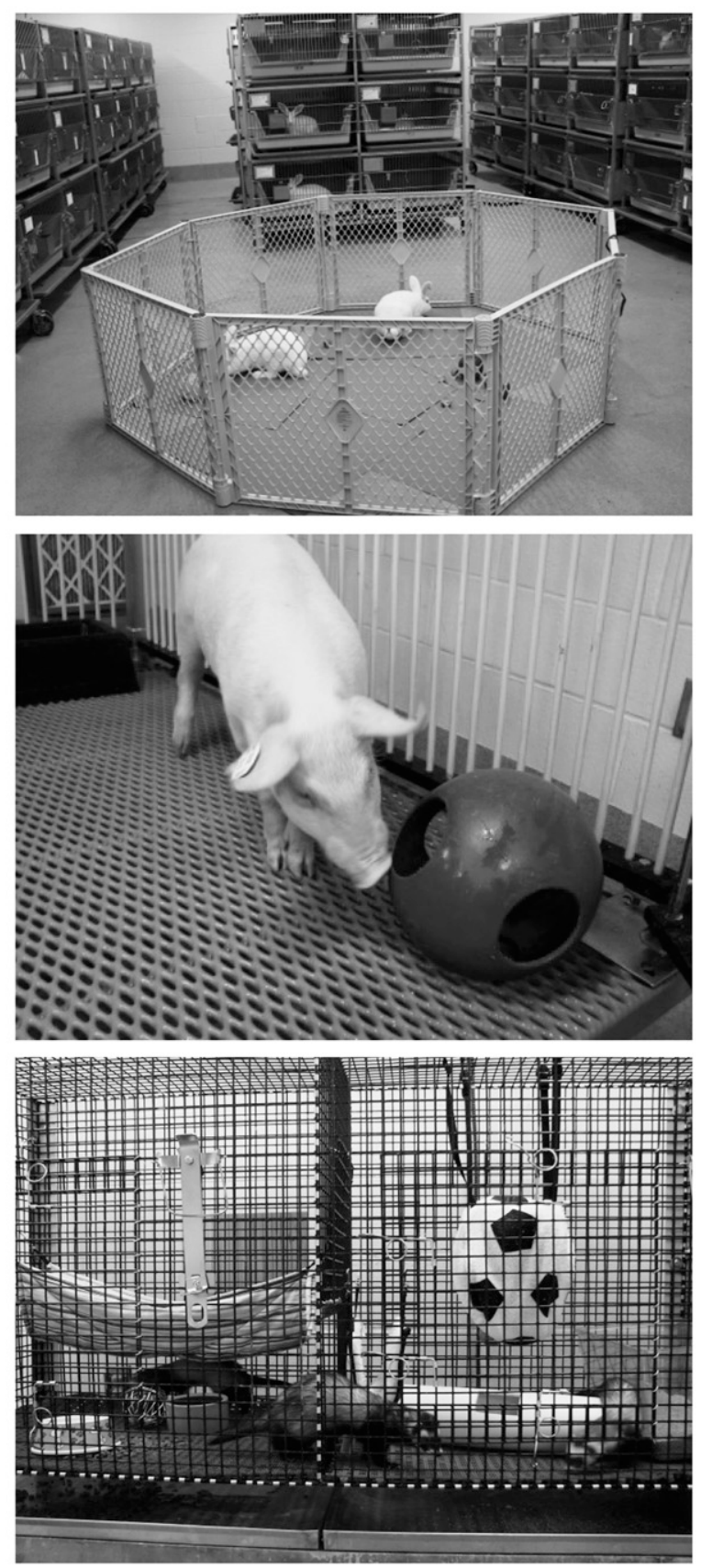

procedures. Laparoscopic oocyte collections for reproductive procedures are being used in both nonrodent and rodent species. In what are termed "look-in" procedures, a small $(1 / 2 \mathrm{~cm})$ incision is made to place just the camera into a body cavity to examine a surgical site that would normally require opening the body cavity. This not only greatly reduces the pain and distress an animal could experience by minimizing the surgical incision, it also reduces the overall number of animals needed by providing multiple time points.
One interesting thing about the advancement in technology is that regulations didn't change with the advances. As mentioned earlier, some primary areas of concern in 1985 were the use of an animal for more than one unrelated major survival surgical procedure and what the definition of a major and minor surgery was. Prior to development of minimally invasive surgery, it was easier to determine a "major" surgery from a "minor" surgery based on the invasiveness of the procedure. But many of the current procedures are done with minimal or no exposure of a body cavity, making it difficult to classify them as minor or major procedures. So the dilemma has been how to apply regulations that were developed 25 years ago to these minimally invasive or interventional procedures. For several years now, minimally invasive procedures have been problematic for both government regulators and research institutions trying to define these procedures. Recently, however, there have been some clarifications and modifications of these definitions, which should help institutions determine whether a procedure should be considered major or minor based on the impact it has on the animal rather than the technique used to perform the procedure.

Looking at even less invasive interventional surgical procedures, access to organ systems is done without ever going into the body cavity, [with] procedures done through the blood vessels, the arteries and veins. The head and brain, the heart, the kidney, and other abdominal organs are now routinely accessed through interventional surgical procedures.

Surgical research using pigs, dogs, sheep, and other large animals helped to develop interventional devices such as intravascular stents for treating abdominal aortic aneurysms (AAA), artificial heart valves, and other devices. Young healthy animals don't often develop chronic conditions such as AAA, so these were created in pig and dog models to evaluate treatments.

Through interventional procedures, neurosurgeons and neuroradiologists can detect a brain aneurysm and repair it using a simple needle stick into an artery and placing detachable embolization coils or Guglielmi detachable coils (GDCs) to fill the aneurysms. Pigs, dogs, and rabbits have been used as aneurysm models to develop new devices and train physicians to perform these techniques.

Interventional cardiology is commonly used to treat coronary artery disease, heart attacks, and stroke. So now, instead of hearing that Grandpa had a heart attack and died, you hear that Grandpa had chest pains, went to the hospital, had three coronary stents placed last month, and shot under 80 on the golf course this afternoon.

Cardiologists are performing interventional procedures and treating arrhythmias (abnormal heart beats) remotely using safer and faster systems. In this case, a mapping and cardiac ablation catheter can be controlled by magnetic fields produced outside the body. This mapping procedure involves a magnetically guided catheter that is maneuvered around the heart remotely to create a 3D anatomical and electrical map to find the area in the heart that is causing the problem. The devices are controlled by the surgeon outside 
the operating room using computers. Figure 3 is a picture of the Stereotaxis Niobe System, a computer-controlled system for performing cardiac ablations. Figure 4 shows an example of a cardiac electrophysiology map (top panel) and the virtual map created; the ablation points create a line and are identified by the labeled dots (center panel). The image on the bottom is the lesion on the interior of the heart.

Imaging procedures such as X-rays, fluoroscopy, and computed tomography (CT) scans have been around for decades but have been improved upon with the advent of digital imaging so that images are clearer and sharper than ever before. Nonradiation-based imaging techniques are being developed using new MRI and ultrasound contrast agents to reduce X-ray exposure to patients and health care workers. Smaller and more focused images (microCT, microPET) and functional imaging (fMRI and PET) are being developed using NHPs and other nonrodent and rodent species. Threedimensional imaging, or 3D CT reconstruction (Figure 5), is being utilized to visualize anatomy and disease states.

Other noninvasive procedures such as Gamma Knife (penetrating radiation to treat brain tumors), lithotripsy (using sound waves to break up kidney stones), and LASIK surgery are now common in human medicine thanks to research using large animals. LASIK is interesting because this is now a household term that everyone knows, but few people stop to think that rabbits and pigs were used to develop it.

What does the future hold for us in terms of procedures and challenges that we may face? Minimally invasive procedures will continue to be developed, including hand-assisted techniques and even less invasive procedures such as singleport and incisionless or natural orifice transluminal endoscopic surgery (NOTES) procedures. So protocols involving incisionless surgery may be something we will deal with more often and [have to decide] how these might be classified (major vs. minor).

Xenotransplantation is being explored and more studies are expected, especially with the increase in transgenic applications to large animal species. Transgenic large animals,

Figure 3 Stereotaxis Niobe computer-controlled system for performing cardiac ablations

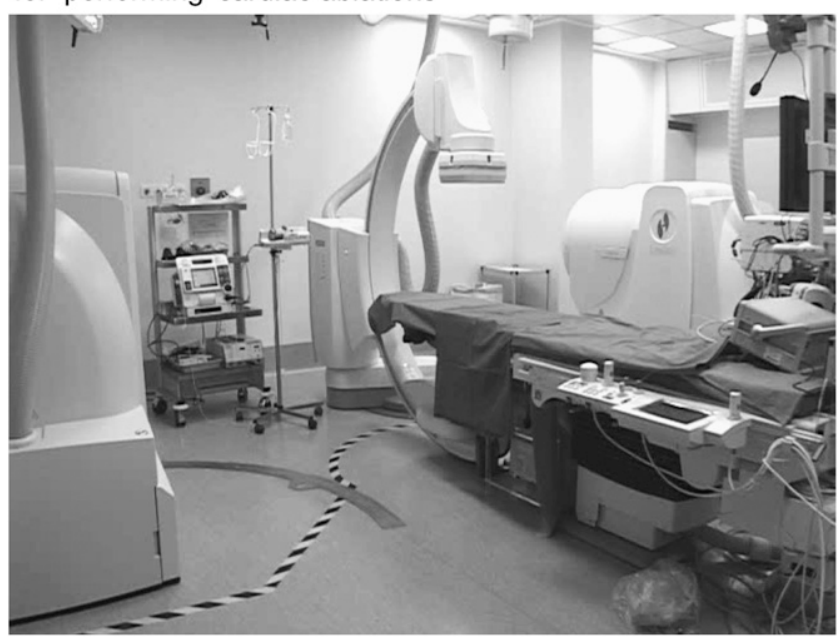

Figure 4 Cardiac electrophysiology map (top), virtual map (center), and lesion on the interior of the heart (bottom)
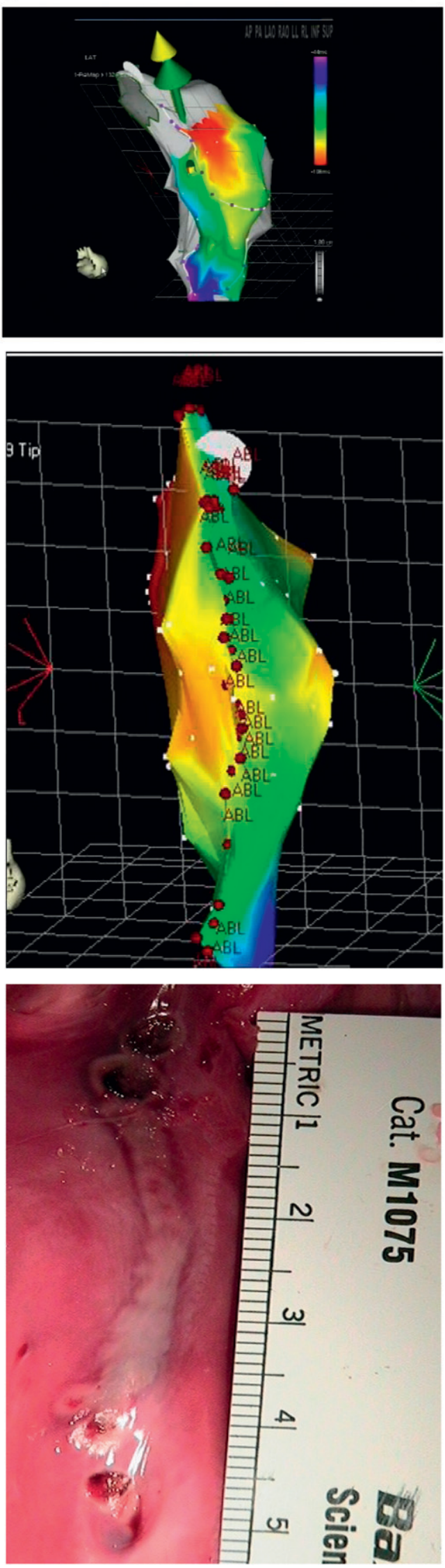
Figure 5 3D reconstruction of pig lower abdomen and limbs

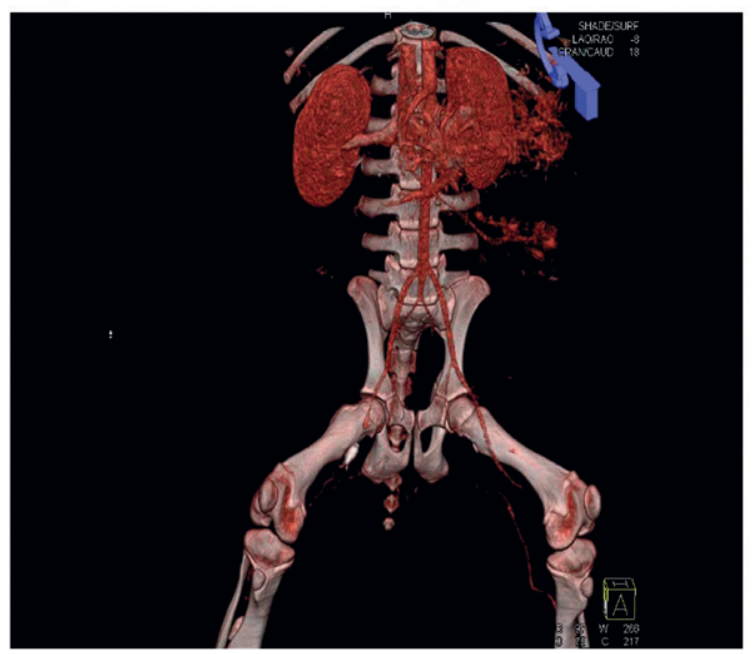

such as the pig, may also be something that we'll have to contend with. And as we discovered during the transgenic boom in rodents, there are clinical and management issues associated with these models.

Nanoparticle research is just beginning to take hold in many facets of biomedical research, and how these particles react and interact in the body may create challenges in how we care for these animals. A recent study demonstrated how gadolinium nanoparticles helped to identify metastasis in a rabbit VX2 tumor model well before the lesions could be seen using other imaging techniques.

And finally, computer-based surgical procedures, robotics, and remotely controlled devices are being developed, and studies and individuals doing telepresence surgical procedures may have to be addressed in the future. Surgeons will have the ability to visualize the operating room, patient, surgical site, and other areas of the room using remote mobile camera systems placed in the OR, and to direct other physicians in the room as well as operate and control devices and instruments from outside the room, from a different building, from a different region, or even from a different country.

\section{Current Standard of Care and Technologies: Rodents}

\section{William White}

he nine US Government Principles for the Utilization and Care of Vertebrate Animals Used in Testing, Research, and Training (page 552), first published in 1985 , not only are as relevant now as they were then but can be considered the ethical framework for the 1985, 1996, and 2011 Guide for the Care and Use of Laboratory Animals. The intent of this talk is to show that improvements in veterinary care and diagnostics linked to improvements in housing and the understanding of the impact of the physical environment on rodents have, over the last 25 years, resulted in improvements in the care and use of rodents in biomedical research.

We have come to realize that the physical environment affects both the animals and the research they participate in. Many physical factors that affect environmental quality have been identified and can be readily measured. We have only begun to understand the ways in which these factors can influence animal health and well-being and the role of animal adaptive processes in adjusting to their physical environment. For the most part, we have focused on extremes in these physical factors. For example, we know that from a thermoregulatory perspective, rodents can survive in temperatures from $0^{\circ} \mathrm{C}$ to approximately $30^{\circ} \mathrm{C}$ and maintain a core temperature within a narrow range that is considered normal. This requires morphologic, physiologic, or behavioral adaptive processes that may change [the animals'] activity, food consumption, and other parameters in order to offset heat loss or gain. At temperatures ranging between $25^{\circ} \mathrm{C}$ and $30^{\circ} \mathrm{C}$ (thermoneutral zone) little or no adaptation is required, and the amount of energy necessary to maintain normothermia is minimized. Rodents will seek to alter their effective ambient temperature if provided structural opportunities such as nest building. Hence, we have come to realize that simplistic prescription of rigid environmental standards, as was the trend in the 1980 s, may not be the only or best way to address the animals' needs. Adaptation to an environment, in fact, may be desirable providing that some consistency can be achieved, that normal adaptive processes are not impeded, and that extreme environment conditions are prevented (Figure 1).

The environment for laboratory rodents and the housing methods used have consistently improved over time. In the 1940s through 1960, there was little attention paid to caging or disease control. A combination of plastic, galvanized metal, stainless steel, and wooden enclosures could be found; and there was very little precision or sophistication in environmental control. Gnotobiotic technology was in its infancy.

In the 1960s through 1985, more standardization of caging design and construction occurred, focused on the use of 
stainless steel and plastic. There was an emphasis on ease of sanitation and disinfection. While microisolation technology had been developed as early as 1958, there was no widespread use of it until the 1990s.

From the 1970s through the mid-80s, infection control was aimed at a facility level, with the goal of preventing infected rodents from entering the facility, usually by imposing some form of quarantine. The presence of adventitious infectious agents was often determined by bacteriologic culture and histopathology, with only limited application of serologic diagnostic methodology. Efficiency of facility operations through the use of a clean-dirty (supply-return) corridor system, which centered around the movement of caging and other materials to and from the cage wash, was an important component of many facilities' infection control programs.

In 1985 infection control was concentrated principally on airborne contamination, with the development and use of cubical containment systems, laminar airflow racks, ventilated cabinets, and biobubbles. The basics of barrier production facility technology were developed in the late 1950s and expanded in application from the early 1960s. By 1985 barrier rooms were routinely used for large-scale rodent production.

During the early 1990s and throughout the subsequent decade, infection control (bioexclusion) became more focused on the group (isolator) or cage level (microisolation caging). While this approach decreased the risk of adventitious infection transfer between groups of animals, the use of cagelevel bioexclusion housing has made the detection of unwanted infectious agents more difficult and less precise. To effectively use cage-level bioexclusion, greater complexity and precision had to be applied to cage processing and disinfection, which has increased the costs and complexity of housing rodents.

The first year that rodents were routinely and reliably available on a commercial basis with an extensive SPF health status was 1985. Diagnostic methodologies for screening a wide variety of adventitious agents had become available, and changes in housing and colony operations at both commercial suppliers and research institutions had improved

\section{Thermoregulation in Rats and Mice}

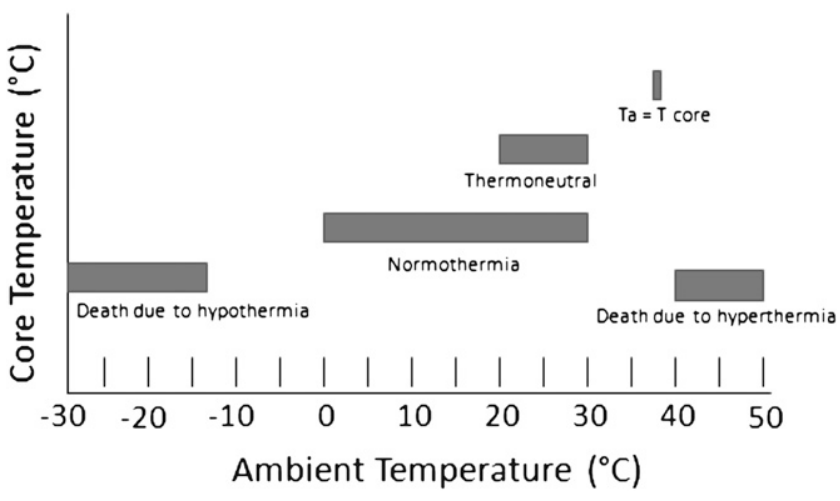

Figure 1 Thermoregulation in rats and mice significantly from the previous decade. There still was substantial use of conventional rodents that harbored infectious agents, many of which had the potential to impact research results and animal health. In 1985 the principal concern was still with infectious agents that produced clinical manifestations. Emphasis was on diagnosing epizootic outbreaks of clinical infection and on eliminating agents that had zoonotic potential. Little effort was spent on eliminating enzootic adventitious infections that had either no or limited overt clinical significance.

In the mid-80s through the mid-90s, there was relatively little transfer of animals between research institutions or the use of wild-caught animals or animals from small breeders with uncertain health status. Biological materials were transferred between institutions, with limited screening of these materials with techniques such as map testing. Intramural colonies maintained for research purposes became fewer in number since reliable commercial sources of rodents of the appropriate health status were readily available.

By the turn of the century, these trends had changed significantly. The use of genetically modified animals had exploded, with large numbers of animals being transferred between institutions and large intramural colonies being maintained. While there was still substantial use of commercial SPF animals, by 2009 they represented only about $50 \%$ of the rodents used in biomedical research. Clinical illness or death of rodents in research institutions has continued to decline over the last 20 years, and latent infection with adventitious agents has become an important consideration because of its impact on subcellular processes and subsequent effects on research results.

Today there is a great appreciation of inapparent infection in rodents and its potential to alter studies. There has been renewed focus on opportunistic microorganisms that cause clinical disease or research interference only under special conditions. There is still confusion as to what importance, if any, should be placed on common human and environmental commensals and whether the costs for control of such agents are an appropriate use of resources under most conditions.

Beginning in the late 1990s and extending through 2010, proactive contamination risk reduction (also known as biosecurity) programs were developed, with an emphasis on minimizing or eliminating microbiological or genetic contamination of research rodents. These programs focus on identification of critical control points in animal facility processes and physical facilities that are intended to ensure freedom from contamination (Figure 2). This process of ensuring the function and efficacy of these critical practices and physical facility components while at the same time questioning and verifying assumptions has led to fewer contaminations and minimized the impact on animal welfare.

Diagnostic methodologies have continued to improve over the last 25 years. As mentioned, in the 1970s through the mid-80s heavy emphasis was placed on pathology and bacteriology due to limited availability of techniques for serologic assessment of the presence of unwanted microorganisms. 
By the 1990s greater use of serology was occurring along with automation of serologic diagnostic processes, as well as the greater use of confirmatory testing by alternative means and the increased use of molecular techniques for verification of test results. At the same time, high-quality commercial reagents for microorganism diagnostics had begun to be commercially available. The use of PCR (polymerase chain reaction) as not only an adjunctive but a primary testing modality is now more commonplace. Both serology and PCR techniques have advanced to multiplexing of assays-hundreds of individual serologic assays can now be completed in a single test well, and thousands of PCRs can be run on a single platform the size of a microscope slide. Real-time PCR has gained increasing popularity, allowing the ability to more clearly eliminate false positive results.

Changes have occurred in sampling techniques for colony assessments over the last 25 years. The original techniques for assessment assumed the use of open cages and unrestricted transfer of agents between individual cages of animals. Sampling from a representative number of animals in a room followed by diagnostic screening for antibodies allowed a reasonable assessment to be made quickly of the health status of a colony consisting of hundreds or thousands of cages. With the increasing use of microisolation caging, sentinel monitoring programs - the use of soiled bedding from multiple cages to expose sentinel animals (which in turn are submitted for antibody detection) - have become commonplace. By 2010, with the use of PCR diagnostics, direct, nondestructive sampling of colony animals using feces and environmental sampling has become possible and has allowed assessment of individual as well as groups of animals. By the pooling of samples, often 10 to 1, large numbers of animals can be sampled economically; and the use of

\section{Figure 2 Biosecurity}

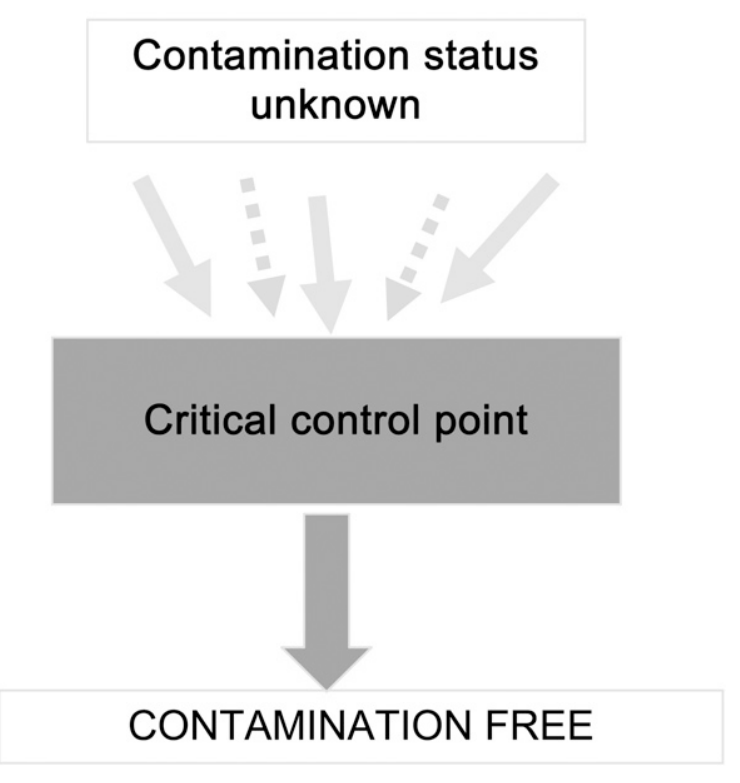

sentinel animals, as well as destructive sampling, can be eliminated. This methodology focuses on antigen detection and hence does not give a historical look at previous infections but only those that are present at the time of sampling. Given the high sensitivity of this technology and its broad ability to be applied against not only microorganisms but complex organisms such as internal and external parasites, there has been a quantum leap in the ability to detect the presence of a wide range of unwanted organisms.

In 1985, imaging of rodents was mostly limited to standard radiographs even though more complex technologies had been developed. Beginning in the 1990s and extending through the present decade, imaging technologies have become a critical component in many aspects of biomedical research. They have allowed animals to be followed over the course of a study in a nondestructive manner serving as their own controls. MRI and dynamic contrast enhanced (DCE) MRI have enabled studies that were thought to be impossible in 1985. Micro-CT has allowed complex soft tissue imaging and studies in respiratory and cardiac gating in rodents that have advanced our understanding of many disease conditions. In vivo bioluminescence and fluorescence imaging using luciferase constructs has enabled detection of cellular and molecular processes as well as pharmacodynamic studies in rodents. Positron emission tomography (PET) with integrated CT (both techniques minimally available in 1985) in rodents has become a clinically accepted measurement of anticancer activity. This technology allows 3D imaging of tracer distribution in rodent body tissues.

In 1985, rodent production was viewed very simplistically whether at a commercial breeder or in in-house colonies. There was an understanding of inbred colony management but very little appreciation of the complexities of outbred colony structure. By the mid- to late 1990s, a new focus on rodent population genetics emerged and new safeguards were put in place to maintain heterozygosity in populations and to link diverse populations of outbred animals. In 1985 there were limited numbers of stocks, strains, and hybrids. With the explosive expansion of genetically engineered models in the 1990s and continuing into this decade, the number of unique rodents has grown exponentially.

In 1985 genetic assessment consisted primarily of genetic monitoring using coat color as well as biochemical and immunologic markers that looked for mismating/authenticity. Naturally occurring mutants were identified by clinical signs, occasionally by histology, and occasionally by clinical chemistry. By 1995 there was regular generation of transgenic animals, and as the decade continued the generation of knockout mice by deleting specific genes had become more and more commonplace. Genetically modified animals sometimes displayed specific and sometimes deleterious phenotypes requiring special housing, care, and monitoring. These unique rodents had the potential to express unusual and perhaps unique phenotypes requiring special testing to assess these abnormalities. Behavioral phenotyping soon became commonplace and an important component in the understanding of these genetically unique rodents. 
Rodent anesthesia and surgery have progressed significantly over the last 25 years. In the 1980s the choices and understanding of rodent anesthesia were very limited. Injectable anesthetics were commonplace as was the use of pentobarbital. Inhalational agents were limited to halothane and methoxyflurane and ether was still used in many institutions. Over the past two decades, rodent anesthesia became more sophisticated, with the use of calibrated vaporizers, new inhalational and injectable agents, and intra- and perioperative analgesics.

Aseptic technique for rodent surgery had been developed, refined, and widely applied in the mid- to late ' 80 s, and improvements steadily occurred throughout the 1990s. Today there is a focus on aseptic surgical technique and the surgical environment, with procedures being done in disinfected laminar flow stations and the maintenance of an aseptic surgical field considered an essential prerequisite. Anesthesia has been tailored to particular procedures, and analgesia and postoperative care are important components of all rodent surgery today.

In the 1980s, complex surgical techniques on rodents were relatively rare. A limited number of vascular catheterization techniques were available. Today, the use of vascular catheterization in rodents is commonplace and has allowed individual animals to be sampled at multiple time points, often without restraint. By contrast, in the 1980s multiple animals would often need to be sampled at a single time point and often without the ability to take nondestructive samples. Today complex microsurgical techniques can be conducted on rodents, allowing them to serve as models for a variety of procedures in humans.

Active telemetry that involves radio transmission of data from implanted sensors has become more commonplace. Passive telemetry using RFID transponders (active transponders are also increasingly being used for this purpose) have enabled precise individual identification of animals, eliminating the need for tattooing, ear punching, and other more invasive techniques. These transponders are also capable of measuring a few physiological parameters. This technology has allowed better record keeping and inventory management of animals, minimizing the unnecessary production and holding of animals and enabling more accurate tracking of individual animal data.

Environmental control and enrichment have progressed steadily since the 1980s. In the early ' 80 s, a few cage space use studies had been done to elucidate some rodent behaviors. Throughout the past 2 decades, more emphasis was placed on evaluating the animals' environment and animal welfare implications that might arise from alterations in that environment. A greater understanding of the use of nesting materials, shelters, and other types of structural enrichment of caging has enabled animals to exercise more environmental

\section{Figure 3 Environmental control and enrichment}
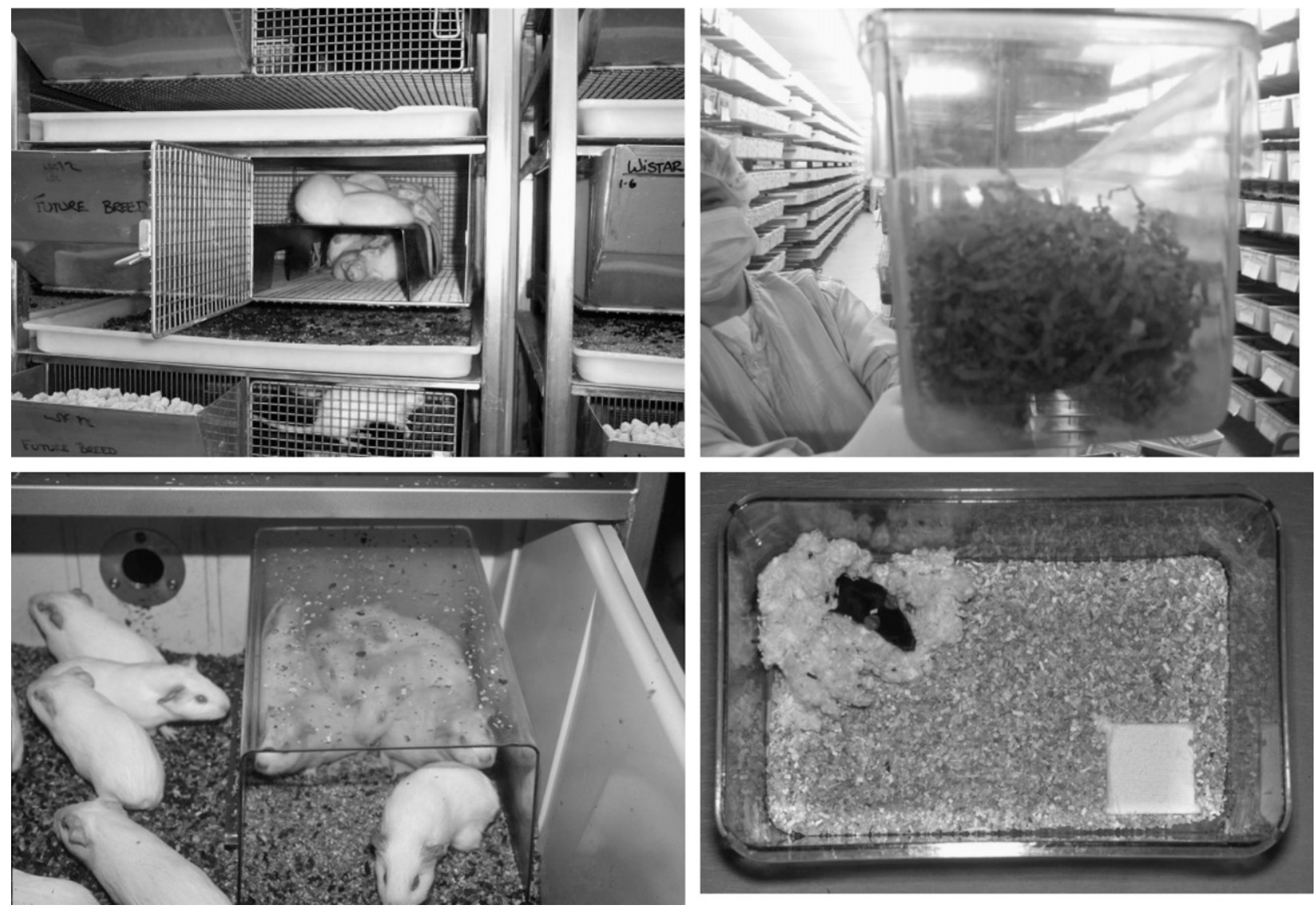


\section{Figure 4 Animal transportation}
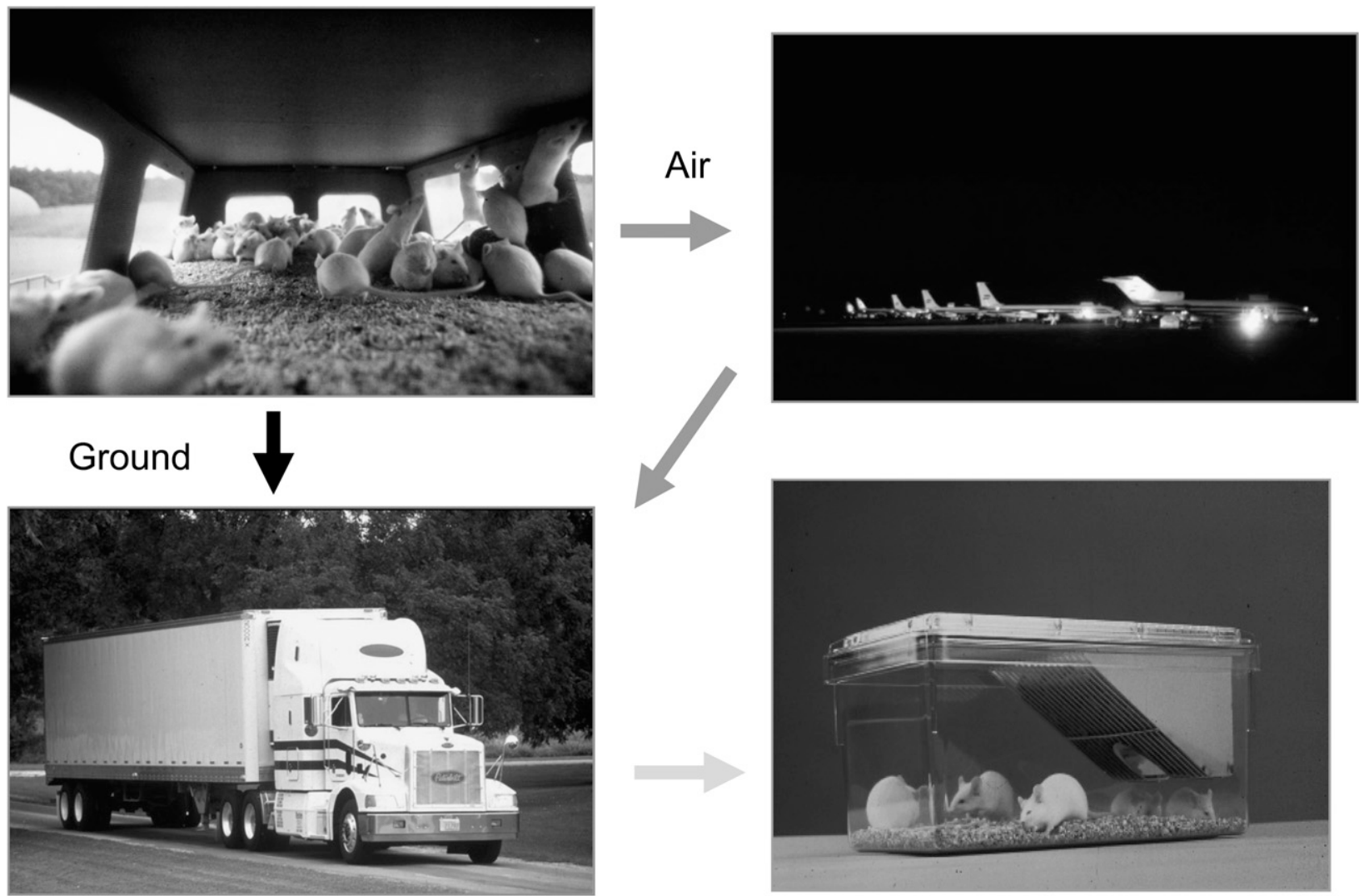

control and to exhibit species-specific behaviors. The importance of common environmental components such as bedding material in rodent thermoregulation has recently been elucidated. Increasingly, the structure of the environment is being tailored to the behavior of the rodents housed within, and the animals are being given greater opportunities to alter the environment independently to fit their needs (Figure 3).

In the 1980s documentation of practices and training was limited. As time progressed, however, the importance of ensuring that there were well-thought-out and consistently applied practices for maintaining the animals' environment and care while in a research facility by all who interact with the animals became evident. The ability to clearly and in a detailed fashion outline all of those practices in a written format that was consistent and could easily be understood by all has become one of the essential means of ensuring appropriate animal care and use. Active training of personnel on these procedures is critical to ensuring that best practices are maintained and animal welfare is not endangered. Failure to agree on what practices are required, failure to describe them in detail in writing, and failure to train all those involved in animal care and use in these parameters is the formula for poor animal welfare and unintended research consequences.

One of the US Government Principles involves animal transportation. The modes of transportation have not changed substantially over the last 25 years. Ground and air transportation of animals remain the principal mechanisms of moving animals from one institution to another (Figure 4). Risks imposed on the health and safety of animals that are being transported have been increasingly minimized by the application of rodent-specific standards for transportation. Organizations such as the International Air Transport Association (IATA) and USDA provide specific guidance for animal transportation. IATA's Live Animals Regulations (www.iata. org/whatwedo/cargo/live_animals/pages/index.aspx), revised annually, have served as a template for not only air transportation but in many instances ground transportation.

A number of improvements in transportation-related issues have occurred over the past 25 years. These include position, temperature, and relative humidity monitors that can be placed in or on the containers; sterile shipping diets/water sources; redundant heating, ventilation, and air conditioning units on ground transportation vehicles; and individual container tracking by bar coding and RFID as well as satellite uplinking on cargo moved by ground, allowing temperature and location to be monitored throughout the route. All these and many other improvements have decreased the risks to animals' health, safety, and well-being during transportation.

While there have been many improvements to rodent care and use over the last 25 years, we still have a lot to learn. It is clear that there are no one-size-fits-all solu- 
tions and that as technology progresses and our understanding of the requirements of rodents increases, our practices for the care and use of rodents will continue to change. The US Government Principles still apply today and will continue to apply in the future. In the end, we need to continue to base our animal care and research practices on sound scientific evidence and to apply appropriate professional judgment that balances the needs of the science with the welfare of the rodents being used to advance that science.

\section{Improving Animal Housing and Welfare}

\section{Brian Hare}

T

o start off I want to do an activity. I want you to turn to somebody nearby and make eye contact, then I want you to reach out and shake their hand. All right, great. Now what I want you to do is stand up. That's great. And now, everybody, if you would sit down. OK, thank you.

That was an example of human cooperation. And the cooperative problem we're trying to solve involves motivating a large group of people to do something together. One of the most important things you can do, if you read the behavioral economics literature, the neurobiological literature, or social psychology, is make people feel good about what they're doing together.

Now, some of you didn't stand up. I'm not going to say who it was. But what if I were to say "Hey, you, buddy, you didn't stand up" as a punishment to that person, and point him out to the rest of the group? [Or] what if the group, as a social norm, didn't like the activity? If we repeatedly did this interaction where you have to stand up and sit down and stand up and sit down, my guess is that we'd have a major drop-off in cooperation. But if everybody agreed that standing up and sitting down and listening to this yahoo up here on the stage was really important, I think you'd have an increase in cooperation.

My point is, I want to try to think of ways to make welfare cool. That's what I want to talk to you about today. I'm going to talk about my own research and draw from the research of other people as well.

Figure 1 shows a PVC pipe and a chimpanzee, Yoyo, living on an island in Uganda; there are no tools in the room, and this is just a normal room that you would keep a chimpanzee in. Now, how many of you think that humans are the smartest species on the planet? [laughter] Come on, be brave, you have to vote. OK, we had some people willing to posit their opinion.

I'm going to show you a video in which Yoyo solves a problem in 15 seconds. My question to you is, can you solve it? There's a million dollars in the tube. It's actually three peanuts, but if you can get them out, I'll give you a million dollars. Yoyo obviously wants the peanuts but there's no tool in the room. [video plays] Yoyo has the solution. Do you have the solution? This crowd, especially, should have the solution. She knows, she's doing it. Yoyo has never seen this problem before in her entire life-as you had not either. We did this at the US Science Festival and little kids were great at it and adults were horrible.

Next I want to reflect on this: Who's the most intelligent or the most highly evolved? In talking about welfare and animal rights, this comes up a lot. What I want to challenge you with is whether it's a continuum scale or do we really believe in evolution? Because evolution, the idea of evolution, is that each species is unique and uniquely evolved to its own ecological niche.

Here's the next test. What I want to do is put up some phenotypic traits that are in humans and also in some of our close relatives, things that aren't necessarily very positivelike gang murder, sexual coercion, sexual taboos, infanticide, and the proclivity to gamble. I want you think about close relatives. How are humans going to stack up? Among chimpanzees, bonobos, and humans, two species have these problems in a big way and the third doesn't. Even though

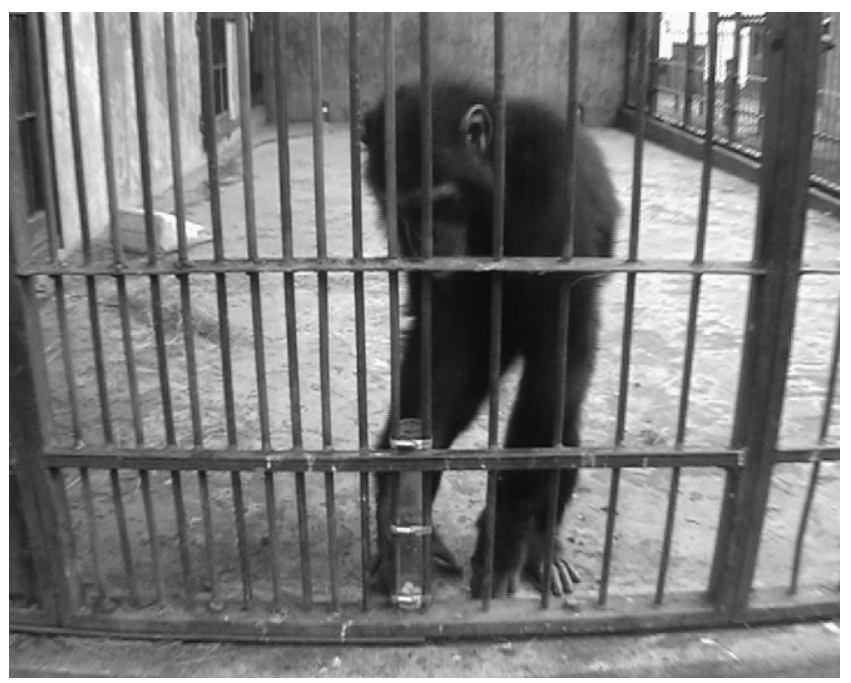

Figure 1 Yoyo the chimpanzee with a clear PVC pipe and a puzzle to solve 
humans can fly to the moon, we have these horrid problems. And the bonobo doesn't. So who's more intelligent? It's very problematic to think about intelligence as the main measure for welfare. Because if you believe this-and maybe I can convince somebody to believe it-maybe you don't need as much welfare for humans as you do for bonobos.

My perspective on welfare is informed by a life on the road. I've worked in lots of places in diverse settings and I've published work on about 20 different species. I've worked in research centers, zoological parks, in sanctuaries, and with wild populations. I've worked with apes, monkeys, foxes, lemurs, dogs, and lots of other things in the cornucopia of life. And being on the road, I'm no stranger to death. I've worked in the Congo, and have seen the worst that life has to offer. Because I work in Africa, I've seen that primates, in particular, are in deep trouble and it disturbs me greatly. So I know that what you guys are doing is incredibly important. And I know that to get people excited about welfare is incredibly important also.

All those influences have informed the way I think about animal welfare. It's something that I've been reflecting on for quite some time. My wife wrote a book about how the thinking occurred. It's called Bonobo Handshake. If you want to learn more about bonobos that we're working with and trying to save, it's in the book.

Today I want to talk about empathy toward animals, and I want to ask specifically, "Are rights NGOs to blame?" And I want to talk about our culture-our culture as biomedical researchers. Are we really trying to improve welfare or are we just trying to get by with defenses? Next I want to talk about "shovel-ready" welfare and a culture of compassion.

I read in the invitation pamphlet that $36 \%$ of Americans, according to a Gallup poll, believe animal testing is morally wrong. Research Today visited Duke and they actually gave more shocking statistics. So this is probably a very optimistic view, I would imagine. The current model, if I read things right in the material sent to me, is that people don't understand the role of animals in research and we must fight radical groups who are brainwashing the public. That means we need to educate and we need to market.

But there is another model that we can consider in thinking about welfare and how to address the problems that we have in promoting our research. This second [model], the biology of empathy, suggests that humans evolved to respond empathically to animate beings, that humans are more empathic to familiar social beings, and that US...culture promotes empathy toward species used in medical tests. The implication is that we need to aggressively flaunt animal welfare standards to the benefit of our research animals themselves. These aren't necessarily exclusive. But I just point out that one may not be more important than the other. One may be more important than the other, but we need to think about it.

We know that humans have a dedicated neurobiology to elicited empathy. It's amazing what will elicit empathy in humans. This [is a video of] a famous experiment by Uta
Frith showing that people can see intimacy in two triangles. I want you to imagine that these two triangles are moving randomly. It's very hard to imagine they're moving randomly because many people see one triangle trying to encourage the other to leave the scary box and then they celebrate the safe exit of one over the other. From developmental literature, from neurobiological literature, there's plenty of evidence that humans can't help but be empathic in ridiculous situations.

What if, instead of shapes, we're talking about dogs? In the United States people view dogs as family members. If you do surveys and ask people about their opinions, they say they're family members. There are 45 million families with dogs. The pet industry is the eighth largest industry in the United States, larger than Hollywood and the only sector to grow during the recession. If people were depressed, they went and bought some dog toys. [There are] doggy daycare [centers] where people have their dogs go to gather and to play. I have people visit my Canine Cognition Center and they are so excited because they are going to [write] on their dog blog about their visit. I mean not their blog but the dog's blog. The most profitable cable stations are Discovery Animal Planet and National Geographic. TIME magazine just had an article about what dogs think and it was the hottestselling issue of 2010.

I want to suggest that this may be a real phenomenon and that people's attitudes about biomedical research may be informed by this. It's not just that people are being influenced by the marketing campaigns of radical animal rights groups. If that's true, then we really need to be aggressive about welfare. We need to talk about it and we need to make it "cool."...

I went on Google Scholar to try to find an article about people's attitudes toward [animal] welfare and I could not find one. I thought, surely somebody's done the research, but I could not find one [article]. I don't know if people think it's cool or not. I can tell you from my own anecdotes, I have two guesses what influences people's attitude: one is, real and perceived danger from radical groups; two is, there is really time-consuming regulation, as we heard from previous talks. It is not necessarily something that is a positive association in people's lives. It's something they just sort of have to do. The next is we're taught-and I have been taught at three different universities - that animals can be unpredictable, they carry diseases, they're dirty, and they're dangerous.

The new OLAW book-this is not a critique, I'm just highlighting this — on chimpanzees [features a] picture of a person in personal protective equipment (PPE) and a chimpanzee grooming, and says, "Human-chimpanzee interactions that permit limited contact in a safe manner can be achieved." I want to contrast this with what's happening in Japan and I'll do that in a minute.

As a professor I have students saying to me things that they wouldn't say to the chair of an IACUC or to other professors. I also was recently a student and a postdoc and heard what people say. This is what I hear over and over and have actually seen written in people's thesis proposals: Macaques 
are violent monkeys. [And] chimpanzees are disgusting because they masturbate excessively and they throw feces....

I sent a work-study student to the library last week. I said, "Go look at the top journals - at least that I knowabout welfare and let's see how much funding people are reporting leading to welfare research." I saw that $2 \%$ of the articles cite NIH funding. If you look at people's CVs, which I have done, and you look for articles in welfare journals in the biomedical community, you don't see any. If they are there and I just didn't see them (I'm doing this in a nonrigorous way), that's something to brag about, that's something that should be on the news.

A solution is maybe to offer work-study students some money because these are fantastic projects for undergraduate thesis people. Another way to get people to do more animal welfare research might be to reward them for doing one welfare publication every grant cycle or every other grant cycle.

The next is to talk about welfare and a culture of compassion. There are two studies that I have to share with you as examples of how cool welfare can be. The first is on dealing with physical pain.

Does anybody know what this bug is? It looks like a stinkbug but actually it is like a leech. It sucks blood and it releases analgesic that makes it impossible for you to detect that it's sucking your blood. A genius biologist used this bug in fake eggs of a species of tern that was highly endangered. The team desperately needed to measure the health of these birds but couldn't because they'd always fly away. And of course, if you catch them, it's very stressful. So they put the little bugs in the eggs. They got up to $2.5 \mathrm{ml}$ of blood and the birds never even knew the bugs were there.

They've done the same thing with bats and with primates. This was the technology they used with bonobosit's very expensive apparatus: a PVC pipe. They just put the bug up against the bonobo as they were grooming the bonobo. They got $2.5 \mathrm{ml}$ of blood within a few minutes and it worked $100 \%$ of the time. They also did this on the smallest primate in the primate order, Microcebus myoxinus. Same thing: they put it in a nest box, they put the bug here in the nest box, and the primate came to the nest box and the bug collected the blood. It worked $100 \%$ of the time and all of the money that goes into needles could go into breeding these bugs. I can assure you that if you did a preference test, the primates would prefer this. Inexpensive and could have a huge impact. How cool would that be when the press release comes out?

Now let's talk about frustration in fur farm mink. This has got to be just about one of the coolest studies that illustrates a preference-based approach to welfare. Mink were given a choice of a room with water, food, space, and toys, and they had to push heavy weights to open the doors. When people had to bet on what minks would want, humans used their own preferences to try to guess what an animal would want without thinking about the ecology that the animal evolved in. What did the minks do? They pushed doors with incredibly heavy weight - double their own weight - to get into water. They didn't care about food. They didn't care about space. And they didn't care about toys. After the mink played in the water for a few days, cortisol went way down. And when the water was taken away, the cortisol spiked to the same level as if the minks were being food deprived. The conclusion is, mink evolved to prefer to spend time and energy swimming. Depriving them of water is the equivalent of depriving them of food. If you understand-and take seriously-evolution, that each species is unique, this is what we have to do: use a preference-based approach.

Finally, I want to take you to Japan, where there is seriously a culture of compassion when it comes to working with chimpanzees - the same species that you can have limited social interaction with. Figure 2 shows the facility that Tetsuro Matsuzawa built because he understands the preference-based approach and he knows that wild chimpanzees spend almost $60 \%$ of their waking hours in trees. So he provided them with metal trees, and there they are spending the exact same time in the metal trees as they do in the wild. And Kohki Fuwa (Figure 3) has spent the last 15 years raising a group of eight chimpanzees. He spends the night with them every night - he goes inside with two adult, 14-year-old males. I saw it when I was in Japan about 4 weeks ago and it was unbelievable, even for somebody who's worked with chimpanzees for 15 years.

They've done fantastic research. Nobody has done what Tetsuro Matsuzawa has done. He's looked at the development of cognition in chimpanzee infants who were raised by their mother but interacted with humans in ways that have never been possible. Not only that, but he did an ultrasound study on the baby while the mother was pregnant. Unbelievable, nobody could ever do that, [but] she voluntarily agreed to let him do this because he had incredible patience and tons of respect for this animal. They have chimpanzees volunteer for event-related potential (ERP) studies, which they are now publishing. Not only that, they are doing eye tracking studies of awake chimpanzees. I was blown away that you could have a 14-year-old male sit in front of an eye tracking monitor. He will hold perfectly still, and for nothing except they are going to hand him some sweet potatoes while he does it. It's going to open up all sorts of doors to these animals' minds that would [otherwise] be impossible.

[Another] example of what it looks like to work with these chimps [comes from] Satoshi Hirata, who has pioneered many of these methods. If you've worked with primates at all, you can't believe [it's possible to] have a 14-year-old adult male sit happily doing an experiment. They just pointed and asked him to sit [and do] a test on how he understands the physical properties of weight. They don't use any punishment. He grew up with them, they're just part of his social group. This is a very different way to do science, obviously. Animals enjoy doing science sometimes....

They've also discovered that chimpanzees spend very little time making eye contact with humans or with other chimpanzees, whereas humans spend the majority of their time staring at eyes. I could never have told you that unless they used this approach. It has huge implications for thinking about mental disorders. 


\section{Figure 2 Kyoto University chimpanzee facility designed by Tetsuro Matsuzawa}

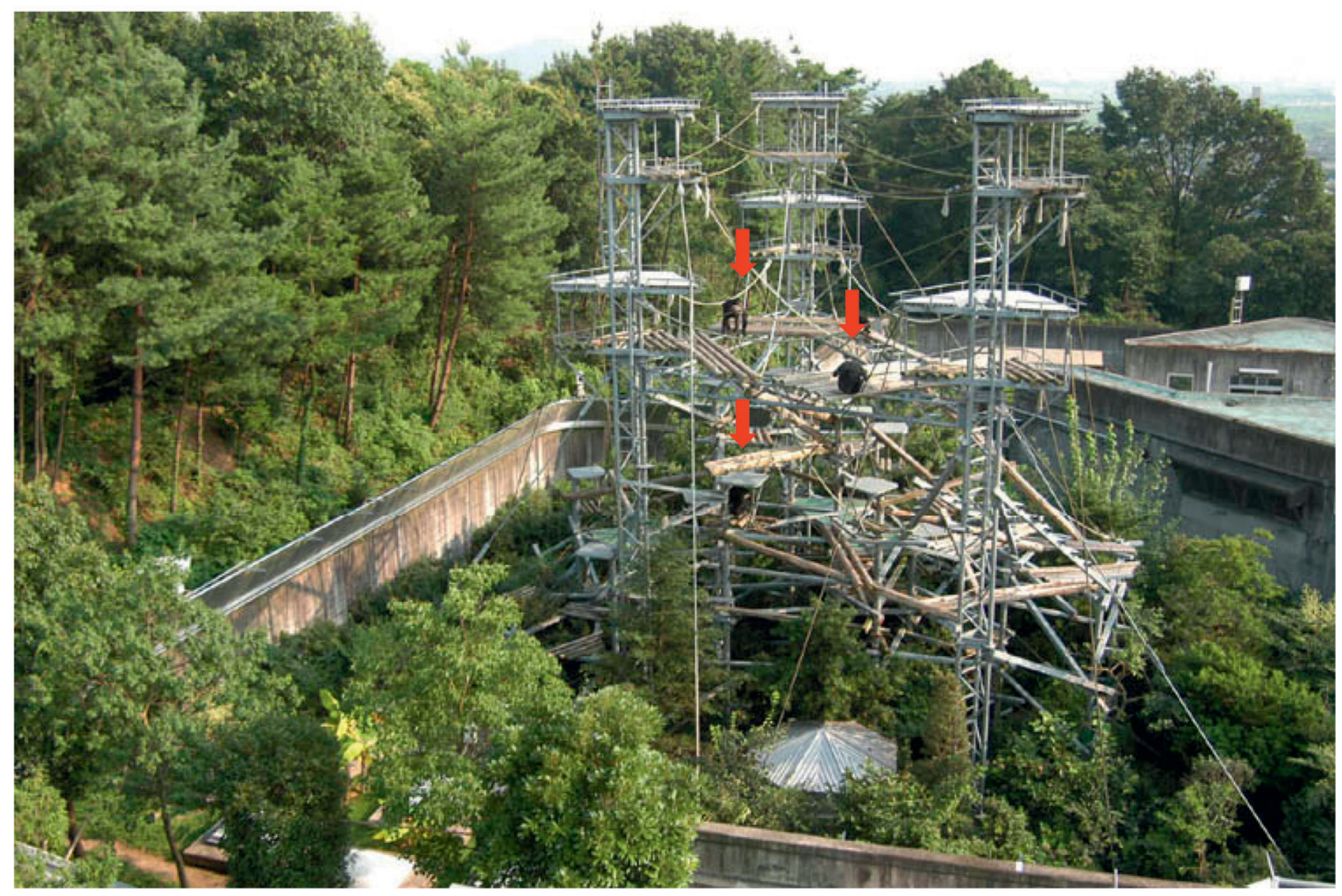

I've tried to adopt, as best I can, the same attitude-a culture of compassion. I work with 1500 animals and none of them are in a cage. I founded the Canine Cognition Center because during my undergraduate and graduate work we discovered that dogs are remarkable: they have evolved to manipulate and take advantage of humans, as you are well aware, in ways that other animals do not.

I'm also excited thinking about effects of domestication on psychology.... There's really no distinction between a domesticated animal and a nondomesticated animal. Of course, we know they have totally different HP axes—what's scary to a domesticated animal will be absolutely terrifying to a nondomesticated animal.

We have upward of 1200 people who bring their dogs in to play games in our lab. If we wanted to take blood samples, we could go to their vets and ask them to let us do whatever we want. It costs me about $\$ 200$ to get some kibble and a couple of cups, video cameras, and so forth. So this is really expensive research, but it's very exciting because, in thinking about dog cognition and dog psychology, we can answer questions that hopefully will allow us to have dogs be much better at all the jobs they do in helping us. There has been a major uptick in having dogs help us with everything from helping autistic children to the traditional roles of people with disabilities.
Finally, in my work with primates, as Pat said I direct a Hominoid Psychology Group. I've worked really hard to have my research have a positive impact in Africa, where the animals I work with live. I do that by supporting the orphanages that bring in these animals when their mothers are killed and by supporting conservation efforts. What do I get in return? I get the largest developmental sample in the world. With chimpanzees, we have over 60 individuals over the age of 5 . If you want to do comparative development, this is the place to do it. I have also the largest sample in the world of bonobos. We know almost nothing about bonobos, even though I showed you the original slide where they're a lot smarter than we are. We have access to two subspecies of chimpanzees. And I get to go to Africa, which is totally fun....

In terms of cost, I don't have to pay the per diem because the welfare organizations pay it. [In] a US laboratory, conservatively for chimpanzees (in the 1997 NRC report Chimpanzees in Research: Strategies for Their Ethical Care, Management, and Use) [it's] \$24 and recent stats say $\$ 40$ or $\$ 41$. If we go to Africa, it's $\$ 5$ a day to offer these guys a fantastic situation.

What about welfare standards?... I'm interested in "extreme" measures of welfare and the best I know of are the "top ten suggestions for captive care of chimpanzees" based on Bill McGrew's decades of work with wild chimpanzees. 


\section{Figure 3 Kohki Fuwa, Primate Research Institute of Kyoto University}
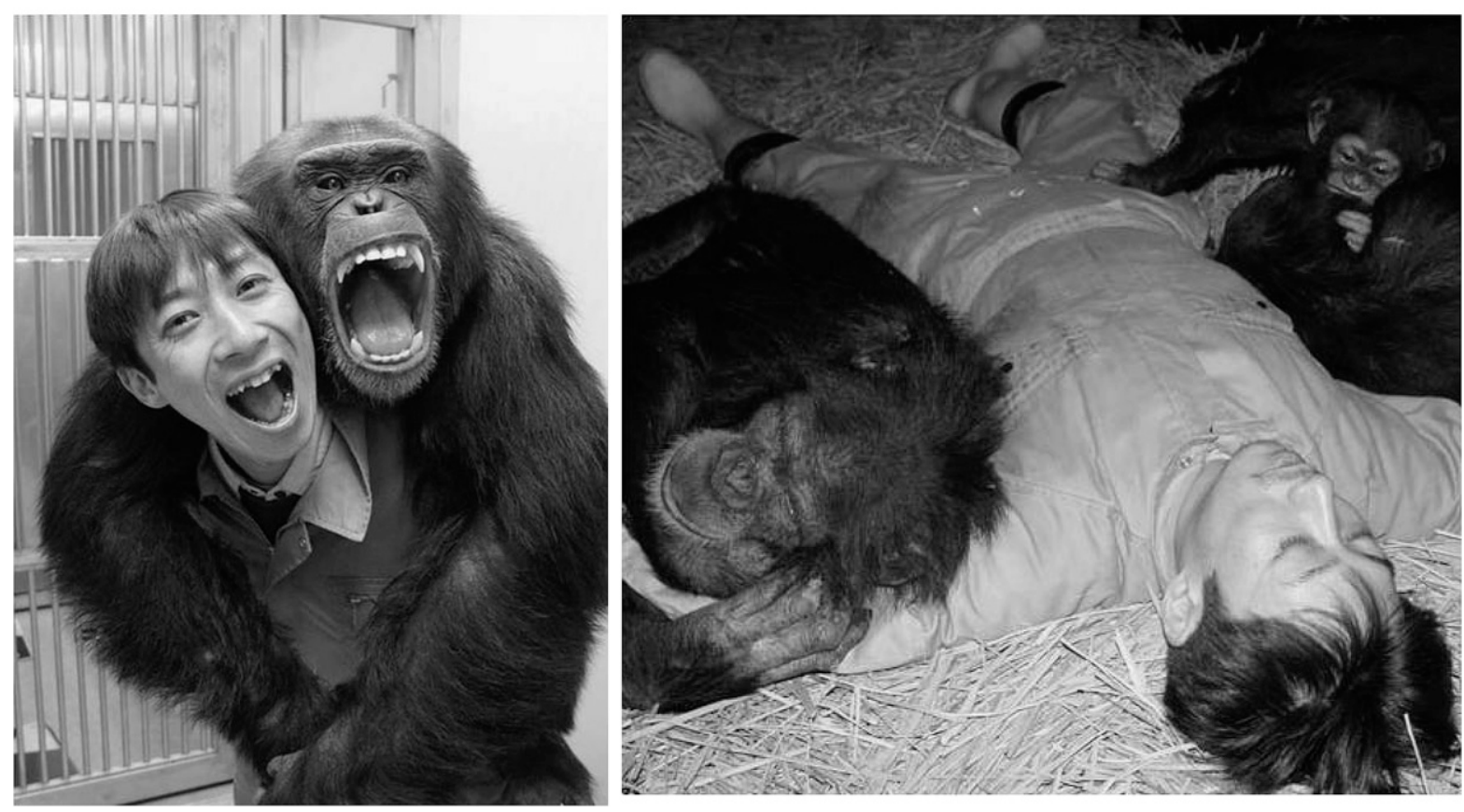

These and, if you go to African sanctuaries, [the animals have] things like equatorial photo periods, rivals and allies for dominance, resources for foraging and processing throughout the day-we've got it all. And we can bring them inside.

We can play all sorts of cognitive games with them and we've had great success in discovering all sorts of things about them in our large-scale cognitive comparisons. We've done comparative developmental research, comparative and developmental physiological research, morphological research, and medical research. We can do diet/tissue samples. I'm involved in the genome project for the bonobo. We have 80 chimps and 35 bonobos for which we've cloned genomes. These are just some examples.

So sanctuaries can be an ideal place for the future of relatively noninvasive genetics, cognition, behavior, disease, morphology, and physiological research for a pittance of the cost.

With the examples I've described, the take-home message is, Can we make compassion for animals cool? Can we make the norm that it's awesome if you're doing welfare? It's not something you need to be shy or embarrassed about or "oh, they punished that guy again, what jerks."

I think there is real reason to consider this alternative hypothesis that humans actually evolved, that biology for empathy is not going to go away-it doesn't matter how much marketing you do, people are going to care about animals. I think throughout the talk you saw that the Three Rs are applied. We need to continue with a preference-based approach in the ecological approach to welfare.

Just some quick ideas about how to get a lot of press about doing good. You guys work at places where they have lots of medical supplies and I work at places where they have none. What if we could get them to where I work? Wouldn't that be a great story? You'd be helping conservation welfare organizations meet one of their biggest needs and helping people at the same time. I don't know how to do it, but it's an idea.

I would also say, if possible, make a distinction between training and working with domesticated and nondomesticated animals. Use domesticated animals whenever possible.

I would much rather see social research-anything having to do with social behavior if it's invasive-done on dogs instead of primates. I think there're lots of things that can be done in dogs now that we know more about their psychology - and they're going to suffer less-even though I love dogs.

I leave you with my last punch line. Let's make compassion cool. 


\section{Veterinary Care: Discussion}

Dr. Brown: We have a few minutes for discussion and questions about all the different topics that you've heard.

Questioner: I really liked that last presentation. It's amazing what can be done. We've been talking about an aging population of chimpanzees in laboratories and it seems like there's an opportunity to study aging noninvasively in the sanctuary setting versus keeping them in laboratories. And to save money. I was wondering if you could comment on that potential for aging studies?

Dr. Hare: Aging happens, of course, your entire life. Most of our [animals] are below the age of 25. Many of the individuals that are participating in aging studies in the United States are much older than that. Obviously there's tremendous potential. It costs about half a million dollars per year to take care of 150 chimpanzees in an African sanctuary, but that could go down substantially depending on your approach and how you house them. If someone made me President Obama for a day, if there were chimps that needed to be retired I would take them back to West Africa, because it's fantastic for the chimps and way cheaper to take care of them.

Questioner: Seems like something funding bodies would be interested in. Thank you. 


\section{Synergy of Working Together}

\section{Dale Schwindaman and John Miller}

Our story is about how two federal agencies worked together to ensure harmonized animal welfare requirements by those two agencies, namely the Department of Health and Human Services and the US Department of Agriculture.

\section{Dale Schwindaman}

thank the organizers for the invitation to be here for this celebration of two federal agencies working together to implement complementary legislation: the 1985 provisions of the Health Research Extension Act, the Public Health Service Policy, and the 1985 Amendments to the Animal Welfare Act. Both legislative acts focused on ensuring humane care and treatment of animals in biomedical research and I believe that, in the end, consultation between USDA and HHS OPRR, which evolved to APHIS Animal Care and NIH OPRR, synergized the aims of the two acts, which, in the end I guess, really fell to John and myself.

In 1987 I had not been working directly in animal care for some time. Then I was tasked to return and take part in the negotiations between the two agencies. Those negotiations were occurring as part of the rule-making process and also as required by the amendment. Implementation of the amendments had been delayed extensively because of the level of public discord. It seemed to me to boil down to a disagreement about the ability of performance rather than engineering standards to ensure protections for the animals used in research. A lot of the acrimony seemed to center on whether the languages and the two legislative authorities could be harmonized.

I came into this effort with a mindset based on certain principles of statutory responsibility, based on the Laboratory Animal Welfare Act (LAWA) of 1966:

- Implementation of legislative intent

- Minimum impediment to research

- Legally enforceable regulations/minimum standards

These ideas were formed as I worked on the very small USDA staff assigned in 1966-1967 to develop regulations and standards; these principles shaped my thinking in 1987 and do so today.

I was working with Dr. Earl Jones, who led the initial Animal Care Staff with advice and guidance from Dr. Frank Mulhern, who was Director of the Animal Health Division staffs (now the Veterinary Services of APHIS). Both of these men, now deceased, were sincere and dedicated public servants. We were faced with a polarized array of stakeholders, and we needed to have a well-thought-out set of common principles and policies.

Most of these policy decisions, as well as the principles we established then, were in place when the 1987 negotiations occurred and affected the course of institutional programs and operating procedures:

- Equitable consideration of polarized input

- Regulations/standards based on available scientific evidence

- Adequate veterinary care requirement fulfilled by doctors of veterinary medicine

For example, we established the policy (shown in the $3^{\text {rd }}$ bullet) mandating the inclusion of a doctor of veterinary medicine to decide the procedures to be used to fulfill the requirements for adequate veterinary care as defined in the Laboratory Animal Welfare Act of 1966. This policy remained in the requirements for IACUCs and in subsequent amendments to the LAWA; it had the effect of extending the influence of professionals whose oath is to ensure the wellbeing of animals.

The impact of respected organizations and individuals in the scientific community who consulted with us as the standards and policies of LAWA 1966 were implemented was considerable and constructive. The initial consultation was under contract to the NRC Institute of Laboratory Animal Resources (ILAR; now the Institute for Laboratory Animal Research). The information based on the experience of these researchers was very valuable, although the minimum standards of veterinary care established by the LAWA required some revision in order to meet the legal limits of the legislation, which were contended through the rule-making process.

Several of the animal protection organizations also made a contribution. In particular, the Animal Welfare Institute under Mrs. Christine Stevens was then, as it is today, a vigorous advocate for animal welfare. Yet this organization led other groups to approach their convictions in a spirit of maintaining research along with protections for laboratory animals, rather than hindering biomedical research. 
So it was that USDA and its disparate constituent groups started out along this road during the 1960s and 1970s with the LAWA and its amendments-strange and somewhat distrustful travelers together. I had the privilege of witnessing over this period a growth in awareness about the concern for laboratory animals during biomedical inquiry. This I believe is the powerful idea that has driven the evolving growth we celebrate in this symposium.

SCAW and PRIM\&R emerged as organizations that bring together scientists and others in the interest of balancing scientific progress with humane care and treatment of laboratory subjects. A very significant evolution occurred with the development of professional specialties in veterinary medicine and the [establishment of] AAALAC accreditation of research facilities program.

In my opinion, the seeds of growth in the scientific community certainly evolved from within as well as from outside pressures. I have a special admiration for the early pioneers in biomedical research who first expressed the need for concern about the care and treatment of the animals usedpeople in organizations like the Animal Care Panel, which morphed into AALAS. New information was also reflected in periodic revisions of the Guide for the Care and Use of Laboratory Animals, which continued the progress to 1985 and after. Throughout the years I worked on the Animal Care staff, our effort benefited from the expertise of laboratory animal veterinarians and other personnel who were willing to support and to train inspectors. And we were able to build what I believe was a cooperative interaction with NIH and also with the Veterans Administration and other agencies, to continue the programs to improve conditions for laboratory animals.

I think Dr. Gene Bingham summed it up pretty well in the AALAS $50^{\text {th }}$ anniversary book, ${ }^{1}$ in the chapter on Recollections. One of the questions asked was, "What were one or two of the major events that changed laboratory animal science?" I called Gene to see if I could use his response, which was "Technological gains. Education: AALAS certification programs, postdoctoral training of veterinarians. Laboratory animal medicine being recognized as a specialty by the AVMA. The Animal Welfare Act-we can thank our adversaries" (p 157). This was a summation of my own philosophy as we approached harmonizing the PHS Policy and the 1985 Amendments to AWA; it describes my mindset when I approached the resolution of the issues between USDA and HHS at that time.

To my surprise, the authorities of HHS and USDA devolved the responsibility for settling lingering issues to Dr. John Miller and me. So I fell back on the principles we had developed in 1966 and 1967 (in the bulleted lists above) and asked myself this question with these principles in mind: Could the "performance standards" carry out the intent of

\footnotetext{
150 Years of Laboratory Animal Science. 1999. The American Association of Laboratory Animal Science, 9190 Crestwyn Hills Drive, Memphis TN 38125; available online (www.aalas.org/association/history.aspx\#50yrs).
}

the amendments while balancing the needs of animals and science? In the care of laboratory animals during biomedical research I have been a strong proponent of performance standards. For example, the concept of performance standards was used in writing the regulations and standards for identifying appropriate dosages and uses of anesthetics, analgesics, and tranquilizer drugs for the 1970 amendments. I have asserted the position that reference to published veterinary knowledge in this area should be the guide for regulations and standards; in that particular case, a performance standard had been accepted.

I want to acknowledge the excellent work by Dr. Richard Crawford of the Animal Care staff in drafting the first versions of the proposed rule making for the 1985 standards and regulations, which did include engineering standards. This was a monumental task, as I know from experience. But by 1987 there was a governmentwide focus on regulatory reform, and that set of standards was rejected at the Office of Management and Budget during the clearance process; it was never published for public comment. As to "outcomes" for the animals from the application of performance standards, there was a paucity of reliable data as to just what actions would produce what level of benefit for the animals.

I looked at the benefits of the 1985 Amendments and concluded that they would provide support and oversight for implementing performance standards:

- ACUCs (with nonaffiliated member)

- Responsibilities of attending veterinarians and institutional officials

- Animal Welfare Information Center

Also, the 1985 Amendments for the first time required actions to promote the psychological well-being of nonhuman primates and exercise for dogs. There was reliable testimony from some scientists that improvement of care in these areas was needed, but little data to quantify actions to accomplish that. In addition, the concept of performance standards seemed to offer the most plausible approach to meet the needs of multiple species.

I believed, and continue to believe, that the best available course and the best prospect for the humane care and treatment of the animals is to rely on the professional integrity of institutional officials, facility directors, and laboratory animal veterinarians and on the activities of the IACUCs and AWIC established in the 1985 Amendments, along with the required regulatory inspections and reviews as oversights. The level of scientific expertise and professional commitment encompassed in these safeguards convinced me that the concept of using performance standards for animal care in biomedical research is feasible and effective.

The other question that needed to be resolved was, What language had to be preserved to support legal action required by the AWA and its amendments? Dr. Miller and I slogged through the numerous issues and were able to harmonize the language that was accepted by the various levels of administrative clearance, including OMB and the USDA Office of 
General Counsel. More than that, both Dr. Miller and I understood that we each must, on behalf of our respective agencies, make sure that the meaning and the language satisfied the needs of both the PHS Policy and the 1985 AWA Amendments, in the context of serving the legislative intents.

I took these decisions very seriously and I know Dr. Miller did as well. I have remained grateful to him personally for his good faith and patience in those negotiations. I am keenly aware of the years, represented in this audience, of successful efforts to balance the needs of scientific inquiry with concern for the care of animal subjects. For that I extend my appreciation and congratulations to all who have been involved for the last 25 years in this important endeavor. I am confident that the progress we have made can and will continue to the benefit of science, animals, and humankind.

\section{John Miller}

see that lots of you out there are from academia and the government. Given the statistics on political inclinations of those groups, I suspect that for this group Ronald Reagan, George H.W. Bush, and Dan Quayle may not be on the list of your top favorite leaders. And they almost certainly don't spring to your mind, I would guess, as having anything to do with animal welfare standards. But in fact, they do, and here's how.

On his second day in office, President Reagan issued Executive Order 12281, which established the Task Force on Regulatory Relief. It was headed at the time by Vice President George H.W. Bush, and its primary goal was to live up to Reagan's campaign promise to reduce regulatory burden. This was the first of several executive orders coming out of the Reagan administration, and subsequently the Bush administration, dealing with federal regulations and regulatory burden.

In 1989, the Council on Competitiveness was created by executive order as a successor to the Task Force on Regulatory Relief, with the same function, again headed by the vice president. The establishment of the Council took place while we were having our discussions about the development of USDA regulations required to implement the 1985 Amendments to the Animal Welfare Act. That's what this is all about. It was far and away the strongest emphasis to date for performance standards rather than specifying the behavior or manner of compliance, which we know now as engineering standards. In looking back, this was the first time that performance standards, performance objectives, came into the lexicon of animal welfare. And they were in the lexicon a lot as we were working together.

It's important to note that compliance by the regulatory agencies with the executive orders and with the provisions and dictates of the Council on Competitiveness is overseen by the White House's Office of Management and Budget (OMB). OMB would play a very critical role in this whole process, a role that most people have no idea they played.

It's been said that timing is everything, and that was certainly the case here. Section 2145 of the AWA 1985 Amendments stipulates that the Secretary of Agriculture shall consult with the Secretary of Health and Human Services before issuing regulations. Now, the Health Research Extension Act, the amendment to the Public Health Service Act that included an animal welfare provision, was passed in 1985 as well, 33 days before the AWA Amendments. That specific stipulation in the AWA was interpreted to mean, and subsequently confirmed in the Congressional Record, that congressional intent was that USDA's Animal Welfare Regulations should be congruent and harmonized with PHS Policy because it came first.

Here's the chronology (Box 1). Getting there was a long haul, as you can see. In December 1985 the Amendments were passed. The first proposal came out in March 1987. Part 1 is definitions, Part 2 is regulations, and Part 3 is standards. You can see how many times they were submitted, revised, and resubmitted. And take a look at the number of comments-I added them up: 36,057 public comments came to the USDA, where Dr. Richard Crawford read all of them. Just imagine having to not just read them but to figure out how you are going to address those comments. In addition, for each of these revisions OMB required another regulatory impact analysis and confirmation that the benefits outweighed the costs of what you were proposing.

How did we do this? Dale [Schwindaman] mentioned that USDA and DHHS devolved a consultation to APHIS Animal Care and NIH OPRR. Department secretaries obviously don't do this themselves, they pass it on down the line. I don't like to think of myself as a lowest common denominator but it kind of looks like I was in this case. But a critically important aspect of this whole process was-Dale touched on it as well - the trust that our agencies placed in us, Dale and me, to work things out. This included significantly more authority than we could imagine these days, or even at that time. We essentially could take positions that we felt met the responsibilities of the agencies.

\section{Box 1 Chronology of Part 1: Definitions, Part 2: Regulations, Part 3: Standards}

- March 31, 1987: Proposed Rules for Parts 1 and 2-7,857 comments received

- March 15, 1989: Revised Rules for Parts 1 and 2, and Proposed Rule for Part 3-5,582 comments on Parts 1 and 2; 10,686 comments on Part 3

- August 31, 1989: Final Rules for Parts 1 and 2

- July 16 and August 15, 1990: Revised Rule for Part 3 -11,932 comments received

- February 15, 1991: Final Rule for Part 3 
In retrospect four principles (Box 2) were the ones we fell back on when negotiations became difficult-and they often did. (Although we never wrote these down, I ran them by Dale and he concurred.) First, we looked back and said that science involving animals is important and it must continue. This was actually stated in the 1985 AWA Amendments. Second, humane care and use and treatment must be provided to animals. Third, wherever possible, regulations should establish performance standards. That one was kind of forced on us. Finally, wherever possible, regulations should be science-based. When we had problems figuring out where we should go, what we should do with this or that thorny issue, these are the principles we fell back on.

Lest you think that our agencies were the only actors in this drama ('though sometimes it was a comedy-it went back and forth), there were lots of other players. I could call them stakeholders-that's a very politically correct term. Depending on whom you talked to, they were actually meddlers, they were thorns in your side, they were a lot of things, but they were, in fact, stakeholders. The scientific community clearly had a big stake in this. We're all aware that NABR and Frankie Trull were very vigilant and strong defenders of the scientific community who often see even minimal intrusion on science as the first steps down the proverbial slippery slope. They also saw, as you do now, performance standards as critical in balancing progress in biomedical research with animal welfare.

For those of you who did not have the privilege of knowing Ms. Christine Stevens, I can assure you that she was just as vigilant and just as strong in pressing for the highest animal welfare standards possible. She in fact played a critical key role in seeing that the 1985 Amendments included the language regarding animal welfare, so she had a sizable stake in the game, so to speak. She was a strong supporter of the 1985 Amendments and not a particularly big fan of performance standards. Enough said.

There were lots of other people and organizations involved including professional societies, Humane Society of the United States, laboratory animal veterinarians (ACLAM, ASLAP, AAALAC), PRIM\&R, and the public. Agency participants included USDA's Assistant Secretary for Marketing and Regulatory Programs, who was the equivalent of our Assistant Secretary for Health - those were the highest-level people, certainly, that I dealt with as we were going through this process-and

\section{Box 2 First principles of consultation}

- Science involving animals is important and must progress

- Humane care and treatment must be provided to laboratory animals

- Wherever possible, regulations should establish performance standards

- Wherever possible, regulations should be sciencebased agencies' Offices of General Counsel, the OMB, and the Interagency Research Animal Committee (IRAC). IRAC is composed of the heads of all the federal agencies that use animals in research and, under Bob Whitney's leadership, it signed off on the final version of regulatory language proposed by the PHS. That acceptance by all of those federal agencies was what OMB needed to press USDA to take that version and ultimately that's kind of what happened.

Dale and I faced a number of hurdles along the path to final agreement. I need to tell a little anecdote related to the legal hurdles. I came to this role, as the old saying goes, fat, dumb, and happy. My first indication that the happy part might be in jeopardy came at my very first meeting in my role as a consultant. This was an initial meeting with the Administrator of APHIS at the time, Dr. Jim Glosser. The meeting was to be held at his office in the big USDA building in downtown Washington. For those of you who did not know him, Jim was a large gentleman and he kind of lumbered when he walked. I have a vivid memory of getting to his office, thinking we were going to sit down and have a discussion. I had made some notes with proposed alternative language to a very early draft of the regulations. Jim said, "Come on, I've got the conference room scheduled so we can spread out a little." So away we went and I remember him lumbering down the hall and me following along. He opened the conference room door and inside the room was a hollow square setup with at least 20 people around it, all of whom, I discovered later, were lawyers. That's when I knew that this was not going to be as simple as I thought it was going to be. So, still fat and dumb- but not so happy.

To enforce policy, you can use the carrot and stick approach. You can take administrative action such as withholding funds and enforce it that way. USDA has to do it through the legal system so they need enforceable standards. That was one of the sticking points we encountered all the time. Was what we were proposing, a performance standard, really enforceable in a court?

The other hurdles were convincing scientists that their work would not be unnecessarily impeded and convincing animal welfare groups that humane care and treatment of animals would result. How did we accomplish this convincing? By spreading the word. OPRR cosponsored sessions with institutions all across the country-lots of them. It seemed like Dale and I were everywhere all the time. The most difficult hurdle was convincing scientists that the world as they knew it was not going to end as we completed this process of consultation and got final regulations.

One last anecdote. Dale and I were at a meeting of a scientific society here in Bethesda somewhere. I cannot remember what organization it was. We were in a breakout session discussing these new Regulations and implementation of the Policy. I will never forget. It was just the two of us on the podium like this and the room was relatively small (because it was a breakout session) and it was packed. Every seat was taken, the walls were lined with people standing, out into the hallway people were standing. They were all scientists and they were all massively pissed off [laughter] 


\section{Box 3 Questions now answered}

- Can performance-based standards carry out the intent of the 1985 Amendments?

- Will professionalism be sufficient to maintain the integrity of implementation?

- Are the oversight mechanisms that are now in place enough?

- Will performance standards be legally enforceable?

- How well will the IACUCs work?

mostly at Dale [laughter]. We feared a little bit for his safety. The scientific community was absolutely up in arms about whatever they had seen. I don't remember what version they had seen by that time. The nitty gritty work of bringing the two agencies' positions together took place in private-at USDA offices, before and after meeting sessions, and yes, even in hotel rooms around the country (i.e., the infamous cheeseburger story).

Dale had mentioned earlier some questions that he had as he was working through this process; they are shown in Box 3. Remember that Dale didn't come to this as a laboratory animal veterinarian, as I did. He is a veterinarian, but his background did not include common species used in research. Performance standards were new to all of us.

\section{Box 4 Why it all worked}

- Good faith negotiations

- Fair and equitable consideration of each others' positions and public input

- Always kept in mind the overarching needs of science and of humane animal care and use

- Trust in the research community

- Two guys who got along

IACUCs were new to all of us. So these were the questions he had in his mind at the time. They actually all have been answered, we both feel, very positively.

Why did all of this work? Why were we able to do it? I think there are several points (Box 4). Good faith negotiationswe always trusted that the other person was dealing in good faith. Fair and equitable consideration of each other's positions and of public opinion - part of good faith negotiations. We always kept in mind the overarching needs of science and of humane animal care and use. Pretty basic, but when you get wrapped up in details, sometimes you really need to step back and look at this very basic stuff. Trust in the research community. We had limited experience with this and it was going to require significant trust. But in the final analysis, it was just two guys who got along. And we got harmonization at last, in 1991, long after the Amendments were passed.

\section{Synergy of Working Together: Discussion}

Questioner: A question that many of us have had for years: Folklore has it that the two of you were locked away in a hotel room and told you couldn't leave until it was resolved. Is that where the hamburger was purchased? [laughter]

Dr. Miller: It was in a hotel room, but I don't recall that there were any threats that we couldn't leave.

Dr. Schwindaman: No, they didn't lock the room or anything [laughter].... But really, the meeting that John mentioned, with the Assistant Secretary of Agriculture and the Assistant Secretary for HHS when we had that meeting after OMB had gotten it and had sent it back, the Assistant Secretary of Agriculture's office was loaded with, as John said, not only your Assistant Secretary for Health, I think the Director of NIH was there, with attorneys, and we had heard- whether it was true or not-that it came out of the Domestic Counsel of the White House through the Secretaries that this controversy had been going on too long. Our Assistant Secretary at that time was Joanne Smith-I don't mind mentioning her name, she was a great Assistant Secretary. The message I got from what she said to me and later John was, Enough is enough. Get it done. That message was there, whether the door was locked or not.

Dr. McCarthy: I can confirm that. The word was passed to the Director of OPRR: "You get an agreement or you may be sorry." We never quite were told we'd be fired but we certainly knew that there was anxiety at the very highest levels. If you two guys had not pulled it off, I probably would have been serving in a very different department, if at all. [laughter] 


\section{Keynote Address: Standing on the Shoulders of Giants}

\section{Charles McCarthy}

usan Silk found a wonderful statement made by John of Salisbury in 1159 that serves as a theme for this presentation. Salisbury said, "We are like dwarfs sitting on the shoulders of giants. We see more, and we see greater distances than they did, not because our sight is superior or because we are taller than they, but because they raise us up, and by their great stature add to ours."

Figure 1 shows acknowledged "giants" in the research enterprise of laboratory animals. They are rightly known in the field as "founding fathers." They were the first persons to insist that the quality of veterinary medicine taught in US colleges and universities must be improved and special additional training be given to laboratory animal veterinarians, because the skills required of them go beyond those of other veterinarians and the knowledge expected of them is more sophisticated than that of veterinarians in other fields.

Dr. Bennett Cohen, on the right, was widely recognized as the leader of the group. He is revered by every senior vet-

Figure 1 Four of five "Founding Fathers" of laboratory animal veterinary care. From left to right: Dr. Nathan R. Brewer, Dr. Elihu Bond, Dr. Robert J. Flynn, Dr. Bennett J. Cohen. Missing: Dr. Robert J. Schroeder

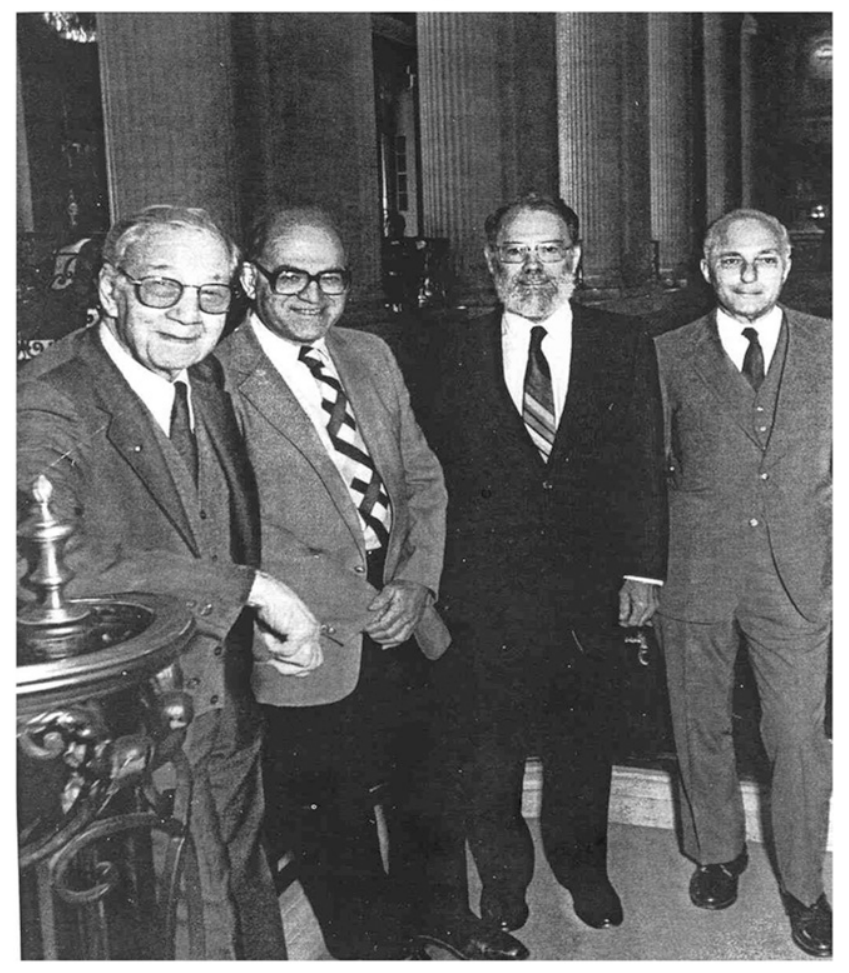

erinarian in America for his contributions to their professional discipline. I had the opportunity to spend some time with him only once. I was so impressed by him that my knees were trembling. To be in his presence was a great privilege. His influence has touched the lives of every person in this room. I think he would be justifiably proud of advances made in the care and use of laboratory animals since his time.

Other leaders have come to the fore in more recent times (Figure 2). Most of you know Dr. John Miller on the left and Dr. Nelson Garnett on the right. The man in the middle is Dr. Alan Sandler who worked in the Office for Protection from Research Risks (OPRR) for many years. Alan was our silent enforcer. He spoke very softly to many institutions informing them that if they did not improve their animal programs their Assurances would be withdrawn and their programs closed. A number of institutions failed to heed his warnings. Two of the most notable were Columbia University in New York and the City of Hope in California. Alan spoke to institutional officials at both and said, "We are withdrawing your Assurance. You may do no more research involving animals until you come into full compliance with the Animal Welfare Act and the PHS Policy on Humane Care and Use of

Figure 2 Three "giants" in the field of research animal care: Dr. John Miller, Dr. Alan Sandler, and Dr. Nelson Garnett

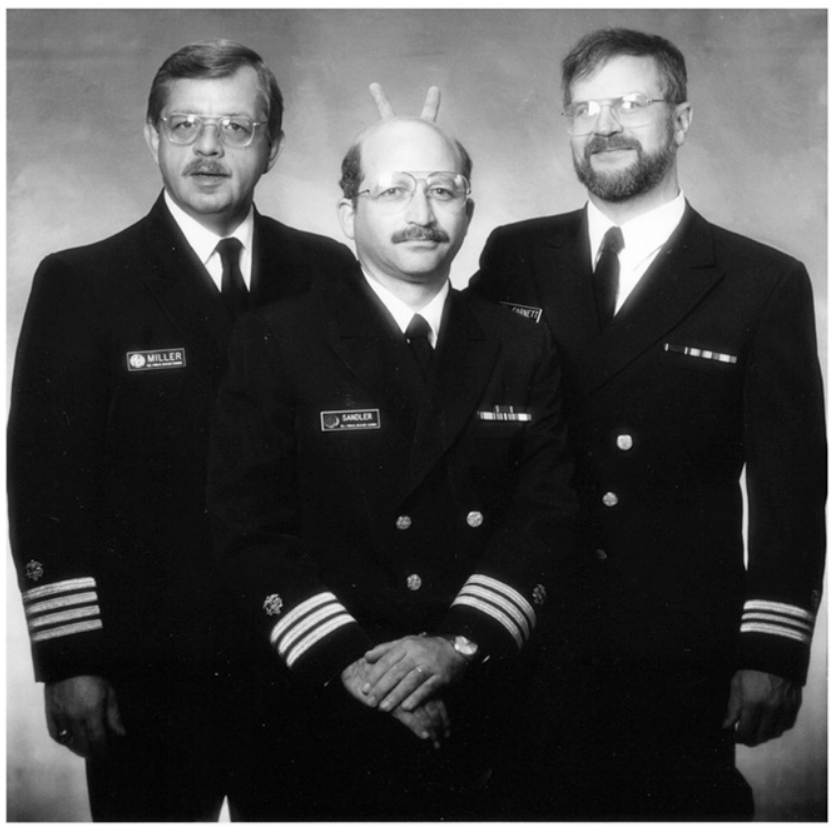


Laboratory Animals." He was a short man with a soft voice, but he carried a big stick that he wielded with high moral principle. He attended many meetings, but always remained on the fringe of each meeting. So far as I know, he never delivered a public talk or lecture. He was welcomed by veterinarians who operated sound programs, and he was feared by veterinarians in institutions with defective programs. I recall one instance when Alan spoke to the Vice President for Research at the University of Pennsylvania. He said, "Here is a list of violations that I found in your caging areas. If they are not corrected immediately, OPRR will withdraw your Assurance and you will be unable to carry out any laboratory animal research." The vice president replied, "Thank you for the warning; we will make all these corrections within a month." Alan replied, "You were warned some time ago. I am leaving in the morning, but before I leave I am going to come here to see if the problems are corrected. If this laboratory is not in compliance by tomorrow morning, your Assurance will be withdrawn." Indeed the laboratory was transformed by the following morning and met the standards of the PHS Policy and the USDA Regulations.

I will comment later about the outstanding contributions of Drs. John Miller and Nelson Garnett. This country owes a debt of gratitude to all three of these outstanding public servants.

Federal regulation of laboratory animals began in 1966. The sad story of a mistreated Dalmatian named Pepper led to the passage of the first version of the Animal Welfare Act that year. The cruel events in Pepper's life were pictorially illustrated in Life magazine. Life was the Twitter of its dayvirtually everyone in America read the stories and viewed the pictures in Life every week. When a picture of Pepper, forlorn and emaciated, appeared on the cover of Life, millions of Americans were thunderstruck. Pepper was a family pet stolen by a dealer in Pennsylvania, resold to a dealer in New York, and sold again to Montefiore Hospital for research. The commercial sale of animals was, until that time, unregulated. The tragic picture on the cover of Life was sufficient to persuade the Congress to enact the first version of the Animal Welfare Act. It was signed immediately by President Lyndon Johnson.

The Act was designed to prevent the use or sale of stolen dogs or cats for research. It remedied a serious problem in this country. The history of laboratory animal research up to that time was "peppered" with cases of theft, abuse, and neglect of laboratory animals. I am happy to say that the purchase and treatment of research animals is on a far higher moral plane today than it was in those days. The Animal Welfare Act was amended and expanded in 1970, 1976, 1985, and 1990. The 1976 version set standards for animal dealers, exhibitors, circuses, and the import of animals. The Act prohibited animal fighting. In the first three revisions the law required "engineering" standards - that is to say, it set forth measurable standards for cage sizes, nutrition, temperature, air changes, and many more. Finally in 1990 the Act was amended to include "performance" standards, requiring human judgment related to the animal's habitat, comfort, and well-being. The Act excluded coverage of rats, mice, birds, and farm animals; some of those exclusions still hold.

I would like to call your attention to the PHS Policy of 1979 , because that is the first policy pertaining to animals that I promulgated. I know nothing of earlier policies. I confess to you that I had little knowledge or training in animal research when I was appointed to be Director of OPRR. Dr. Donald Chalkley, my predecessor, had suffered a severe stroke and was forced to retire.

Dr. Tom Malone, NIH's Deputy Director, chaired the committee that selected me to succeed Dr. Chalkley. He briefed me on my new responsibilities and talked to me at length about human subjects and protections that must be afforded to them. As I was leaving his office he said, "Oh, by the way, you will also be responsible for the humane care and use of laboratory animals." That was the only instruction given to me concerning laboratory animals.

When I returned to OPRR, I looked up and read the only PHS Policy that was under consideration at that time. It was still in draft form. It had been drafted primarily by Dr. Chuck McPherson. Because of his illness, Dr. Chalkley had never promulgated the Policy. One of my first acts in OPRR was to publish the McPherson Policy_although I had only a little understanding of what I was doing! Colleagues assured me that McPherson was an honest man, a good vet, and a wise regulator. In many ways it was a wonderful Policy: it emphasized good animal husbandry, appropriate housing, appropriate feeding, clean water, air circulation, clean cages, and competent veterinary care. However, I soon came to realize that the Policy had a serious shortcoming: it lacked enforcement authority for OPRR and [articulated] no authority for the institutional veterinarian or an institutional committee.

As I became familiar with my new responsibilities, I found many violations of the Policy. But I also found authority built into the Policy for enforcement. Dr. Chalkley had implemented sound practices only by the force of his own personality. He wrote long-hand letters to university presidents and directors of research centers, saying: "There are serious abuses going on in your institution. You have failed to meet your moral obligation to correct them. This office is holding you personally responsible." The letters were blunt and often offensive. His letters would provide details of the abuses and give directions for correcting them. Typically the university presidents would write to the Director of NIH and say, "Who is this fellow Chalkley who has told me how I am to run my university? He has insulted me."

When complaints about Chalkley's letters reached a crescendo, Dr. Ronald Lamont-Havers, Associate Director for Extramural Affairs, called me to his office and instructed me to stay every evening after hours and rewrite Chalkley's letters. I was instructed to take all the insults out of them, remove all of the direct blame heaped on university presidents, readdress letters to persons directly in charge of laboratory animals, and say very politely, "There may be some shortcomings in your program for the care and use of laboratory 
animals. Please review the allegations (detailed below) and if you confirm any shortcomings please improve your program as soon as possible. NIH stands ready to assist you." With this strategy, negative mail in the NIH Director's Office diminished. I am not sure that my efforts did much for the well-being of animals, but I think they advanced my career. At least the McPherson Policy gave us standards that institutions were required to follow. After we promulgated the Policy cases began to flood into our office.

Perhaps no case was more vexing than the so-called Silver Spring Monkey Case. It was that case that made me acutely aware that the PHS Policy, although it set wise standards for the care and use of laboratory animals, contained virtually no investigative or enforcement provisions. When the Silver Spring case broke in 1983, I was in Los Angeles investigating a major abuse of human subjects. A research investigator had forged a fake approval of his research project by an IRB and was conducting research in dying cancer patients. He was injecting spinal stem cells into leukemia patients without approval from the local IRB or consent from the subjects. All of the 18 patients died. We believe their lives were shortened by the illicit research procedures. I started going through records before 6:00 $\mathrm{AM}$ and spent a long day interviewing nurses, doctors, and IRB members. I sought in vain someone to defend the accused.

I remember returning to my hotel with a headache from a long, discouraging day. I turned on the TV to ABC News and saw photos of a police raid on a primate laboratory in Silver Spring, Maryland, only a mile from my home. Tired as I was, I realized that I was not watching a movie but that somehow the cameraman was already inside the laboratory when the police raid began. That indicated to me that the raid was set up from inside the laboratory. The commentator stated that the laboratory was filthy and smelly. The animals there were deafferented-the nerves in one of the shoulders of each of the primates had been severed. This process simulated the results of a stroke, and the purpose of the research was to determine which, if any, treatments could restore or rebuild any muscular activity in the inactive shoulders and arms. The cages were filthy and covered with rust. $\mathrm{ABC}$ cameras were panning all around the laboratory even as the police were confiscating records attached to each cage.

Before I went to bed that night I contacted OPRR and, with help from Bill Dommel, planned a site visit to the Silver Spring laboratory. That was a long eventful day for one person simultaneously struggling to enforce Regulations for the Protection of Human Subjects and the PHS Policy on Humane Care and Use of Laboratory Animals. Although the violation of the rights of humans seemed to be even worse than the deafferentation of primates, the press put the primates on page 1 of the LA Times and the story about abuse of humans on page 19 .

The research investigator in the Silver Spring Case was Dr. Edward Taub. The person who admitted the cameraman ahead of the raid was Mr. Alex Pacheco, one of the founders of People for the Ethical Treatment of Animals (PETA).
Taub had hired Pacheco as a summer employee to care for the animals in the Silver Spring lab. Pacheco took many photos of the handicapped monkeys. The animals were taken to the apartment of Ingrid Newkirk, another of the founders of PETA. This was a clear violation of zoning laws and good animal husbandry. It endangered the health of residents and the health of the primates. It turned out that the investigating police officer was also a member of PETA. Ironically, a short time later, the animals were stolen from Newkirk's apartment. Eventually, following an agreement not to press charges for theft with another group sympathetic to research animals, the animals were returned by truck to NIH where they were temporarily housed in animal facilities in Poolesville, MD.

Dr. William Raub, then Deputy Director of NIH, took over the case. It was often called the Raub-Taub Case. Disputed issues were tried in court six or seven times. The case finally went to the Supreme Court. Dr. Taub was found guilty of failure to consult a veterinarian at periods required by the PHS Policy. Taub contended that he knew more about the care of primates than any veterinarian. Clearly that was not the case. The courts never decided whether the neglect of the laboratory and the poor condition of the animals was Dr. Taub's fault. Many believed that he had been "set up" by PETA. Eventually the animals were shipped to a laboratory in Mississippi where they lived out their lives.

The Silver Spring investigation was hampered from the beginning by the need to get Taub's permission to examine his laboratory and documents and by lack of cooperation from the Maryland police. OPRR learned the value of including enforcement procedures in the animal welfare policy. The Silver Spring Monkey Case stimulated OPRR to consider revisions to the PHS Policy-it was clear that it required more "teeth" to permit investigations and sanctions of violations of the Policy. It was also clear that someone or some group at each institution must have authority over research involving laboratory animals.

In 1984, the self-styled Animal Liberation Front (ALF) broke into the University of Pennsylvania Baboon Head Trauma Laboratory. They trashed the laboratory and stole 60 hours of videotape of animals involved in a "whiplash injury study." ALF turned the tapes over to PETA, which edited them down to about 20 minutes and produced a film called "Unnecessary Fuss." The title came from the NIH Director, who had used those words to describe the fact that PETA created an unnecessary fuss by refusing to turn over the tapes to OPRR and by showing an inaccurate altered version to congressmen and senators as well as to state officials and private associations of various kinds. PETA twisted the director's words to mean that concern about cruelty to animals was an "unnecessary fuss." The 20-minute tape was narrated by Ingrid Newkirk.

The research study involved animals given simulated whiplash injuries. Whiplash is an injury to the neck and shoulders of humans involved in rear-end automobile collisions. The purpose of the study was to learn how best to treat whiplash in baboons and ultimately in humans. When an animal was subjected to a sudden jolt pictures of the animal's 
head were taken from many angles to develop an understanding of what muscles and nerves were injured in whiplash accidents.

The 20-minute film claims to show that a large number of animals were subjected to whiplash procedures. Eventually OPRR was able to obtain the original unedited tapes. The master tapes demonstrated that only one animal had been subjected to whiplash injury, although pictures of that animal were taken from many angles. Many believe that inflicting whiplash on even one baboon was not justified. All agree that PETA was dishonest in editing the film in such a way as to make it appear that the injury was repeated over and over again. Many erroneous statements were included in the commentary associated with the edited film.

Films from another camera focused on the surgical table in the laboratory and showed several surgical procedures. The camera was fixed on the table so that it was not possible to see the faces of those conducting the surgery. In reviewing those films, OPRR noticed that puffs of cigarette smoke often billowed across the operating table. Clearly that created a danger for the surgical patients (baboons), but also for the humans involved because oxygen tanks were nearby. We also noticed that the smoker (whose face we could not see) extracted the cigarette from a pack in the hip pocket of his trousers. Of course University of Pennsylvania rules prohibit smoking in all surgical units on the campus. When OPRR interviewed Dr. Thomas Gennarelli, he reached into the lefthand hip pocket of his trousers and extracted a cigarette. He lit it while standing under a "No Smoking" sign in the conference room. It was clear that he had lied about not smoking in the surgical unit.

OPRR found indisputable evidence that PETA had deliberately altered the evidence and repeatedly lied about it in public and before Congress. PETA also withheld evidence from OPRR for many months. OPRR used that information to discredit PETA with the members of Congress. We also found that Dr. Gennarelli had deliberately lied about smoking in the surgery to OPRR investigators. Eventually under pressure from OPRR, the University of Pennsylvania closed the Head Injury Laboratory. OPRR moved on to revise the PHS Policy.

The difficulties we had with these cases made us acutely aware that the PHS Policy needed revision. Because President Reagan was strongly opposed to issuing any regulation not required by law, we did not even consider attempting to create PHS regulations. Reagan had imposed a 5-year moratorium on hiring. The moratorium left OPRR with the responsibility of overseeing the care and use of laboratory animals throughout the country, but we did not have even one veterinarian on our staff. Consequently OPRR turned to Drs. Tom Wolfle and Bob Whitney who, among their many other responsibilities, were in charge of the NIH laboratory animal facility in Poolesville, MD. Over a period of several years, they answered every request submitted to them by OPRR.

I have asked Drs. Whitney and Wolfle to join me on the podium. Clearly they rank among the "giants" on whose shoulders rested the implementation of the PHS Policy.
Their concern for making sure that programs throughout the country were in compliance with the PHS Policy made it possible for OPRR to meet its responsibilities.

OPRR assembled a small committee to review the PHS Policy. Our experience with the Silver Spring Monkey Case and with the University of Pennsylvania Head Injury Case and dozens of other less publicized cases had made us acutely aware that we needed to establish local authority in each institution that conducted research involving laboratory animals.

OPRR formed a drafting committee to create a revised PHS Policy. Carol Wigglesworth, a young staff person in OPRR, served as secretary for that group. Wolfle, Whitney, and Wigglesworth did the heavy lifting. Eventually Carol produced a final draft of the Policy. For that work, and for her subsequent leadership as Director of the Office of Laboratory Animal Welfare, I consider Carol to be another one of the "giants" of the laboratory animal community. I served as Committee Chairperson, but they did the work.

I was very busy in those days giving speeches and leading workshops that combated the philosophy that is was immoral to use animals in research. If that philosophy had been translated into a governing policy, it would have dealt a mortal blow to biomedical research. OPRR took a middle ground. It strongly opposed any activity that imposed unmitigated pain on laboratory animals. On the other hand, OPRR strongly opposed unnecessary and crippling restrictions on research involving animals. Many groups insisted that all research involving laboratory animals should be forbidden. That position, had it prevailed, would have undermined medical progress for both humans and animals. My liberal arts training in philosophy proved to be exceedingly valuable. We made it clear that OPRR stood for protection of the rights of human research subjects and for the well-being and proper care of laboratory animals used in research. That approach is now widely accepted.

As soon as the Policy was drafted, OPRR began to implement it. Before coming to OPRR, I had served as a legislative liaison to Congress in the NIH Division of Legislative Analysis (DLA). I knew a few members of Congress and had worked with many congressional staff members. Working through staff, I was able to persuade Congressman Doug Walgreen to allow us to test the Policy for a year before it was added to the Public Health Service Act. He said something like this: "I think that is a splendid idea. Try the Policy first, report back to me how it works, and then we will change the law to incorporate the tested Policy." We did try the Policy, which included for the first time the requirement that each affected institution establish an IACUC. True to his word, in 1985 Rep. Walgreen shepherded amendments to the Public Health Service Act through the Congress, establishing the PHS Policy which required institutions to establish IACUCs.

That same year, 1985, Congress amended the Animal Welfare Act. The new law required the USDA to issue revised regulations. It also required the USDA to consult with DHHS. After several failed attempts by the USDA to publish 
regulations that met the spirit (as well as the letter) of the AWA Amendments, Dr. John Miller took the matter into his own hands.

John had come to OPRR only a short time before. At last the hiring freeze was over and OPRR was able to recruit a veterinarian. Dr. John Miller came for an interview. I had written about 24 questions to be answered by candidates for the position. He informed me that he thought he could tolerate working with me, and he told me a joke. I laughed so hard I could not finish the interview. John turned out to be another "giant."

Dr. Miller secretly redrafted the failed USDA regulations; persuaded affected government agencies to endorse (without actually seeing the draft) his revised version of regulations, which emphasized performance standards; and won the approval of the Office of Management and Budget, which, in turn, persuaded the Secretary of Agriculture to publish Miller's draft for comment. Public comment strongly favored the Miller version, and it became the final Animal Welfare Act Regulation that is operative today.

If John Miller had done nothing but draft the USDA Regulations, he would be worthy to be called a giant on whose shoulders laboratory animal veterinarians and scientists are able to see untold future opportunities. USDA had struggled for 4 years to draft and promulgate acceptable regulations. USDA failed. John Miller single-handedly accomplished the task for them in a matter of a few weeks. His performance was unprecedented and amazing.
John Miller accomplished many wonderful tasks at OPRR, and one of them was to bring soft-spoken Dr. Nelson Garnett on board. Nelson eventually replaced Miller (who moved to AAALAC) as the Director of the Animal Division of OPRR, and then succeeded in separating the Animal Division from the Human Subjects Division. He was the first Director of OLAW. The new office quickly became known as a helper and teacher. Institutions began to bring their problems to OLAW instead of hiding them. The smooth transformation of the Animal Welfare Division into OLAW qualifies Nelson Garnett as one of the giants in our field.

In closing, I wish to salute Nathan Brewer, Elihu Bond, Robert Flynn, Bennett Cohen, Robert Schroeder, Alan Sandler, Carol Wigglesworth, Helen Gordon, John Miller, and Nelson Garnett. Each of these persons made "giant" contributions to the humane care and use of laboratory animals.

I was privileged to work with most of these giants. They are among the best public servants I have ever encountered. It is my privilege tonight to recall and brag about the wonderful people who have made it possible for all of us to celebrate the $25^{\text {th }}$ anniversary of the Public Health Service Policy. Thank you.

\section{Acknowledgment}

I should like to acknowledge my dear friend Amy Chuang, who prepared the PowerPoint slides for this presentation. 


\section{Reflections on IRAC and the US Government Principles}

\section{Robert Whitney}

hen I was invited to this meeting and saw that Tom and I were asked to speak on this topic, I called him and said, "This reminds me of Jeremy Bentham." For those of you who don't know about Jeremy Bentham, he lived from 1748 to 1832 . He was an English jurist, a philosopher, and legal and social reformer, best known for his advocacy of utilitarianism and the ethical treatment of animals.

Bentham was also considered the godfather of the University College London. In his will he left them lots of money and he also left his body to be used for biomedical research with the stipulation that after they were finished they would do whatever was necessary to make an "autoicon" of him (that's a euphemism for being stuffed) and so they did. His body was dissected as part of a public anatomy lecture and afterward the skeleton and head were preserved and stored in a wooden cabinet (Figure 1). The skeleton was stuffed with hay and dressed in Bentham's clothes. It's normally kept on public display at the end of the South Cloisters in the main building of the University College London. But on the $100^{\text {th }}$ and $150^{\text {th }}$ anniversaries of the college it was brought to the meeting of the college council where it was listed as "present but not voting." The auto-icon had a wax head as Bentham's head was badly damaged in the preservation process. The real head was displayed... on the floor between his feet. However, it was stolen on several occasions and so was later removed and is now locked securely away.

Jeremy Bentham has lots of quotes in literature. The one that is most meaningful to those of us that care for and use animals in biomedical research is what he said when he was speaking about animals: "The question is not 'Can they reason?' but 'Can they suffer?'" We all know the answer to that is yes and a big part of our job is to make sure that we do our best to eliminate, minimize, or mitigate any suffering.

Now I want to say a few things about the Interagency Research Animal Committee (IRAC), which evolved from the Interagency Primate Steering Committee (IPSC) established in 1975. Let me take you back to the early 1950s when the Salk polio vaccine finally went into large-scale production. After a couple years of production, in 1957, they produced a batch of "inactivated" vaccine that wasn't inactivated. A number of people died of polio. Up to that point monkeys had been used sparingly in biomedical research and testing. But the polio vaccine was made with monkey tissue and the safety testing was done with rhesus macaques. So the FDA really kicked up the testing and also the numbers of tests-every batch of that vaccine was tested in rhesus macaques before it went out for distribution. Let me just give you an idea of what that meant. In 1957, 200,000 macaques were used for polio vaccine production, and in 1958 another 200,000. All were imported from India.

My NIH boss, Joe Held, described working at one of the large quarantine facilities during that early period of polio vaccine. The losses were terrible. In the 20 years of polio vaccine safety testing, over 1.2 million rhesus monkeys were imported to the US from India. A high percentage of these died because of tuberculosis and shipping problems. It was a terrible time and it was obvious that this couldn't continue. It was also obvious that there were real issues for the United States associated with using nonhuman primates in research.

The international community began working on the Convention on Trade in Endangered Species of Wild Fauna and Flora (CITES), and their recommendation was to list some primate species as endangered and all other primates

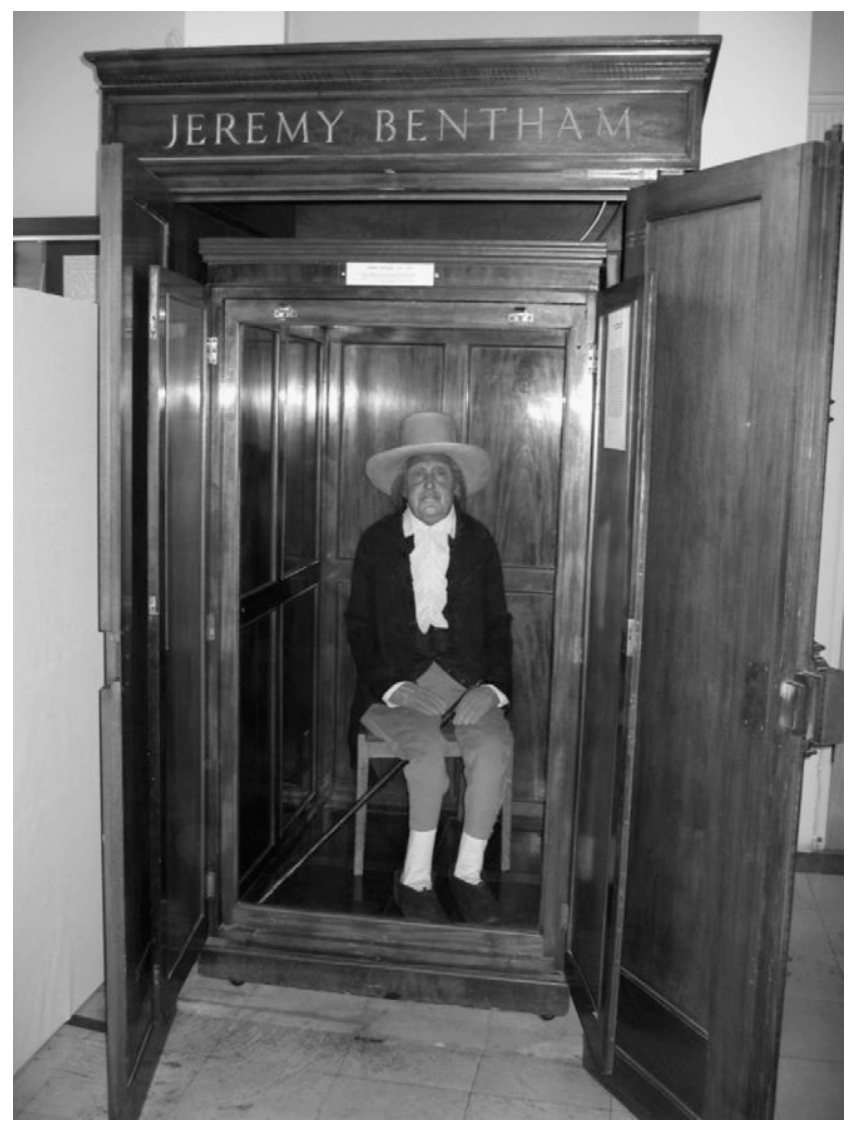

Figure 1 "Auto-icon" of Jeremy Bentham 
as threatened species. This meant it would be difficult to obtain nonhuman primates from their country of origin, some not at all and others under only extreme circumstances. We in the US biomedical community were looking at a freight train coming down the road and we had to start making some decisions about what to do. In the early 1970s, just before the CITES was signed (in 1975) Joe Held and Jim Vickers, who worked for FDA and had been running their primate programs for a number of years, got together with a few more people from NIH and the FDA and started talking about what we could do to somehow get under control and coordinate the issues surrounding the large number of primates that had been available in the past but wouldn't be available in the future. It was obvious that this issue affected the entire research community, not just $\mathrm{NIH}$ and FDA. Other federal agencies began to be added to the committee.

In 1974, the director of NIH officially appointed the IPSC, composed of representatives of nine federal agencies:

- $\mathrm{NIH}$

- $\mathrm{CDC}$

- FDA

- Office of International Health

- National Institute of Mental Health (which was in a different category than NIH)

- Department of Defense

- Environmental Protection Agency

- National Science Foundation

- VA

A year later, the Assistant Secretary for Health approved the Interagency Primate Steering Committee under his aus- pices with NIH as the lead agency and Joe Held as the chairman. The IPSC put together a National Primate Plan, which was published in 1977. A number of government-sponsored breeding projects were initiated as we were desperately trying to get out of the business of importing wild-caught primates from countries of origin rather than those that had been bred in captivity.

Fortunately, another thing was happening during this period. There had been an evolution of changes in the testing of polio vaccine and a test was eventually developed using mice. The horrific number of rhesus macaques that had been imported for the last 20 years went down to about 30,000 a year. By 1975 the Indian government had dropped the number of rhesus monkeys that they would allow to be exported every year to 30,000 , which was about what we were using in biomedical research. The next year, 1976, they dropped it to 20,000, and in 1978 the Indian government banned the exportation of their nonhuman primates. That's still enforced today.

The IPSC activities began to really pay off, getting together a plan, getting several agencies involved, and getting funding to start breeding colonies both in the country of origin and in the United States.

By the 1980s the folks in Europe were developing international regulations and policies for all animals used in biomedical research.

A forum was needed in our country to address these potential new international issues and their effect on biomedical research using animals in the US. Using the IPSC member agencies as a starting point in 1983, the then Assistant Secretary for Health, HHS, Dr. Ed Brandt, directed Dr. James Wyngaarden, the Director of NIH, to expand the IPSC to include all interested federal agencies in the renamed Interagency Research Animal Committee (IRAC).

\section{Thomas Wolfle}

I am tickled that Jeremy is with us here tonight. Besides being a lawyer, as Bob said, he made his fortune from his father who was a plutocrat in England and he became known as a philosopher. What he liked to do as a philosopher was coin words, merge words from Greek and Latin and come up with words like "international" - he coined that word and we use it today. He also coined "postprandial vibrations"-afterdinner talks. Being a pragmatist and utilitarian, he believed happiness trumps sadness. He believed good for the most, sadness for the least, that sort of thing. He was a very outgoing person. So in deference to Jeremy, we're going to try to keep this very brief and keep you happy.

I'll start 28 years ago. Bob ended [his talk] at the end of the IPSC. About a year before, it had morphed into IRAC. Joe Held and Bob were working in both Strasburg, France, and Geneva with the CIOMS (Council for International Organizations of Medical Sciences) under the World Health Organization on broad international principles for the use of animals. They also worked with the Council of Europe, which was creating new policies on animals of all kindsfarm animals, research animals... Joe had integrated these organizations and was working hard with them. It became clear, as Bob said, that we were trying to build relations with exporting countries. We were forming breeding colonies in foreign countries like Puerto Rico and Peru and working with the local populace helping with public health problems and that sort of thing to try to create stable colonies of nonhuman primates.

With the experience coming from the Primate Steering Committee we knew that the source of primates was going to be a continuing problem and that international relations were not going away. So the Primate Steering Committee was expanded both in content and in charge. One of the issues it started working on was the US Government Principles, modeled largely after the CIOMS Principles, which Joe brought back to the IPSC and ultimately to IRAC, and those committees worked on it. Having every federal agency in the United States that uses or supports the use of animals working on a 
document, and ultimately endorsing it, carried a lot of weight and that's exactly what they did.

As I recall, these Principles were never put out for public comment except by the agency representatives that worked on them but they were quickly incorporated in the 1985 PHS Policy and the 1985 Guide for the Care and Use of Laboratory Animals. They're a super set of policies, read them some time (page 552). They're in your program book-they're very succinct, one page. The CIOMS principles are seven pages long, eleven policies, nine subsections, and three appendices but they say the same thing. They're all right there together. The US Government Policies, rather than go into things like nutrition and housing and husbandry like CIOMS did, rely on the Guide and the PHS Policy for those issues. So they could afford to be succinct and direct and to the point. I love them. 


\title{
Animal Welfare and Scientific Research: 1985 to 2010 October 26, 2010
}

\author{
Perspectives of PHS Funding Agencies: Session Introduction
}

Susan Silk

- oday we're going to learn about the organizations that support our science. I'm honored and grateful that today's speakers took time away from their research, their students, their offices and their patients to travel to Bethesda to be part of this meeting. This morning's session will be about the policies and philosophy of the Public Health Service organizations that support biomedical research. I'm going to introduce all the speakers in the order in which they will speak.

First we will hear from my professional home, NIH, where Dr. Sally Rockey is Deputy Director for Extramural Research. She was recently confirmed in this position after serving as Acting Deputy Director — congratulations, Sally. Dr. Rockey received her PhD in entomology from the Ohio State University in 1985. She went to work for the USDA and quickly rose through the ranks to become Deputy Administrator for the Competitive Research Grants and Awards Management Unit in the Cooperative State Research, Education, and Extension Service, and then she became Chief Information Officer.

It is also my pleasure to welcome Dr. Tanja Popovic, Deputy Associate Director for Science at the Centers for Disease Control and Prevention (CDC), where she previously served as Associate Director for Science and as Chief Science Officer. She joined CDC as a Fulbright fellow in 1989. Since then she has served as the Chief of the Diphtheria Reference Unit, Chief of the Epidemiological Investigations Anthrax Laboratory, and Co-Director of the WHO Collaborating Center for
Prevention and Control of Bacterial Meningitis. She has been a WHO consultant for bacterial meningitis and diphtheria in Russia and throughout Africa. She led the CDC laboratory during the 2000 anthrax events. Dr. Popovic is the CDC Institutional Official for both human and animal research subjects. She has many more awards and credentials, too many to list.

And finally, we will hear from Dr. David Jacobson-Kram, who received his $\mathrm{PhD}$ in embryology from the University of Connecticut in 1976. He served as a senior staff fellow at the National Institute on Aging, on the faculty of the George Washington University School of Medicine, and at Johns Hopkins University Oncology Center. He has also served the Environmental Protection Agency as a geneticist in the Office of Toxic Substances and as acting Branch Chief in their Office of Research and Development. Then Dr. Jacobsen-Kram entered the private sector, serving as Director of the Genetic Toxicology Division of Microbiological Associates. Microbiological Associates changed its name to BioReliance and David's responsibilities expanded to include oversight of the Mammalian Toxicology Program and the Laboratory Animal Health Program. He also served as Vice President of the Toxicology and Laboratory Animal Health Division. Dr. Jacobson-Kram is now Associate Director of Pharmacology and Toxicology at the FDA Office of New Drugs. We're very lucky to have David here with us today - I understand he had a tremendous fall this weekend so don't hug him or shake his hand too vigorously.

\section{Use of Animals in NIH-Supported Biomedical Research}

\section{Sally Rockey}

'm going to talk about the National Institutes of Health and our perspective. I want to give you some context and then talk about how our policies support the appropriate care and use of animals and about NIH's support of model systems and alternatives.
$\mathrm{NIH}$ is an agency of the US Department of Health and Human Services (our sister agencies CDC and FDA are represented here, too). NIH is one of many organizations in DHHS that has an interest in animal research. It is composed of 27 institutes and centers, 24 of which have grant authority 
and intramural research programs. Among our many institutes, most are derived around a disease condition or an organ system; for many disease conditions there are reasons to use animal models to study those diseases.

Our mission is science in the pursuit of fundamental knowledge about the nature and behavior of living things and the application of knowledge to extend healthy life and reduce the burden of illness and disability. With a mission statement like that it's a joy to come to work every single day.

$\mathrm{NIH}$ has two components. We are a science organization conducting research, that's our intramural research program. We have about 6,000 scientists on the NIH campus, around Washington DC, and across the nation. Our budget is currently $\$ 32$ billion, and the intramural program is about $10 \%$ of that. Our other part is our extramural program, which supports about 3,000 institutions across the nation. We calculate at least 300,000 scientists are supported, in part or in total, through NIH grants and contracts. About $83 \%$ of our budget goes to our extramural program.

$\mathrm{NIH}$ is a very large agency and a very diverse agency. We fund individuals in every state in the nation. We also fund many projects across the globe. As I noted, we have an intramural laboratory, which is very involved in animal research. Our extramural support goes to universities, medical schools, hospitals, and research institutions around the world as well as to small businesses and some institutions in the private sector. Every type of organization is involved in NIH programs.

A critical component to our program is to support investigator training. We have such training here at the NIH in our intramural program as well as a very large program in our extramural program. [All who] are involved in animal research [must undergo] required, intensive training on the proper use of animals in research.

Another part of our mission is fostering communication of medical health science information. We support the development of information technology and share our medical resources through the National Library of Medicine's PubMed and its public access to publications on biomedical research. Toxline is another resource that the National Library of Medicine (NLM) provides.

In order to accomplish our mission NIH supports the use of animal models, and we have policies to support the proper care and use of animals. This is very, very critical. The use of animals brings with it a great responsibility to ensure their welfare and to minimize their use wherever possible. The oversight of research is shared among a number of different departments in the federal government, between the USDA and the NIH Office of Laboratory Animal Welfare (OLAW), and also through the voluntary peer review of AAALAC. So there is a "trifecta" of organizations that support the oversight of the use of animals.

The responsibilities of each of these organizations are different. In NIH, the Health Research Extension Act and the PHS Policy on the Humane Care and Use of Animals apply. NIH's oversight is over live vertebrates and we support NIH- and PHS-funded research. We have Animal Welfare Assurances and a system of self-monitoring. That means that the organizations we support have written Assurances with us and are responsible for monitoring the use of animals. If something happens, there can be a loss of funding.

With the USDA, the Animal Welfare Act applies. The department has responsibility for federal animal welfare regulations, [which cover] warm-blooded animals excluding most rats, mice, and birds. (When I was at the USDA it was always interesting to talk about chickens because we did research on chickens and the oversight of birds was, at that time, in question.) The USDA deals also with breeders, dealers, exhibitors, and research facilities. This oversight brings additional and broadened aspects to their program. They do unannounced inspections and have both administrative processes and the authority to levy fines when there's a problem.

The Association for Assessment and Accreditation of Laboratory Animal Care (AAALAC) provides voluntary accreditation. They use reference resources in their peer review. Institutions and organizations volunteer to be site visited by AAALAC and may lose accreditation if AAALAC finds serious problems.

Our PHS Policy requires that an institutional program of animal care and use is in place, and part of our oversight is to ensure that the local institutional policies are appropriate and in line with our own policies. Universities and other grantee organizations must have policies in place to receive funding. They must also have (1) an IACUC appointed by their CEO or president, (2) an Institutional Official who's responsible for the care and use of laboratory animals, and (3) a written Assurance with OLAW. So, again, this is an assurance and compliance system and not a regulatory system. We enforce self-regulation; institutions self-report to us. It's important to us to hear from them if there's a problem, but we also have authority for compliance site visits and have other oversight mechanisms.

The Health Research Extension Act gives NIH the authority to place requirements on research institutions. It requires animal care committees, and it requires applicants, before the award of a grant, to provide assurance of training for all involved with animal care-this training component is very, very important to us. It also requires in the application a statement of the reasons for the use of animals in the funded research. We want to see that animals are used only when absolutely necessary and in the most appropriate way.

When an individual or organization applies to NIH for a grant, if it involves animals a discussion of animal care and use is required in the application. There are five points. The applicant must justify the use of the animal, the species, and numbers to be used, and must use the appropriate number to minimize use whenever possible. There must be a description of the use. There must be a description of the veterinary care provided; this is always an issue in review. It's very important to have procedures in place that minimize discomfort, distress, and pain, and applicants must also discuss the method of euthanasia. 
OLAW provides guidance and interpretation of the PHS Policy on the Humane Care and Use of Laboratory Animals and it supports educational programs and monitors compliance. This is a very important part of what we do involving our assured institutions and the PHS funding components. It ensures the humane use of animals in our research, in our testing and training, and contributes to the quality of what we do in our research.

NIH also supports animal model systems and alternatives. I want to say a bit more about our intramural animal care and use program and extramural support of animal research. Virtually all of our institutes that have an intramural component-24 of them-have animals in their research programs. Almost 50\% of the NIH-funded grants and contracts that we support involve animals. As this is a major part of the research program here at NIH it is very important that we have appropriate compliance and oversight of this process. For the intramural program in 2009, we used 1.3 million animals; $81 \%$ of them were mice but there were more than 20 different species-fish and frogs, rats, guinea pigs, rabbits, hamsters, nonhuman primates, birds, dogs, cats, pigs, and sheep. There's a variety of animals supported here in the intramural program, and NIH has a number of resources available if you are looking for model organisms for biomedical research. Our website provides information on model organisms, both mammalian and nonmammalian models including Arabidopsis, the mustard plant.
We also have a number of programs that support the development of nonmammalian models and the improvement of mammalian models. At the National Center for Research Resources (NCRR) the Biological Models and Materials Research Program supports research to develop and broaden the utility of models including cell culture, nonmammalian model organisms, and nonbiological systems such as mathematical modeling or computer modeling. These are examples of our interest in alternatives to the use of animals.

The NCRR Laboratory Animal Science Program supports animal research-related resources and research training through awards and grants contracts and cooperative agreements. Through its Division of Comparative Medicine, NCRR awards animal research grants and resource grants to develop, characterize, and improve mammalian animal models for human disease and study. NIH is interested in both improving mammalian model systems and offering nonmammalian and nonanimal alternatives. The NLM has another resource, the Bibliography on Alternatives to Animal Testing (ALTBIB), which [includes] a search engine to identify resources on alternatives to the use of live vertebrates in biomedical research and testing. On the ALTBIB web page you can identify the sources, put in keywords, and find available alternatives.

Much of the program for today focuses on biomedical research using animal models and how it has advanced knowledge to find prevention, cures, and treatments for the many serious diseases and conditions of humans and, of course, of animals.

\section{Global Impact of Animal Research on Infectious Diseases: A CDC Perspective}

\section{Tanja Popovic}

am going to discuss the global impact that animal research has had on infectious disease, with a CDC angle. I will provide a historical perspective, but primarily focus on the science and the key scientific contributions that have made an impact on prevention of infectious diseases globally. I have selected several infectious diseases for which there is visible progress and clear and specific examples of accomplishments due to animal research. Some of my discussion will involve regulations and policy as well as CDCspecific work.

Obviously, not everything is about infectious diseases and a few key accomplishments that are noninfectious diseaserelated are shown in Box 1, denoting the years and recognition with the Nobel Prize. Some of those accomplishments are making it possible for people to live their lives to the fullest today.

The work on animals started many years ago but it continues to be amazing. When it comes to infectious diseases, a very specific and major accomplishment based on animal research is the development of vaccines, and it is hard to point to a vaccine that has not been "touched by an animal" in some way. Six of those diseases are shown in Box 2.

Every year, 21/2 million children worldwide do not die because their infections and death are prevented by vaccines. Unfortunately, the same number of children still die from vaccine-preventable diseases - over a period of 10 years, that is 25 million children. Each life is precious. We frequently hear about individual tragedies of five or ten people being killed in an accident; now imagine almost 7,000 children a day dying — or not dying because of the contributions that were available through animal research.

There are many other areas in the infectious disease worldfrom pathogenesis studies to mechanisms of immunity, testing of new antimicrobials, and development of monoclonal antibodies - that have been recognized as major accomplishments. In all of these studies animals have not necessarily always been visible, but they are the unsung heroes. Charles McCarthy told a compelling story about Pepper, the Dalmatian 


\section{Box 1 Selected discoveries related to noninfectious diseases and conditions}

\author{
1921 Insulin* (dog, fish) \\ 1929 Vitamins supporting nerve growth* (chicken) \\ 1942 Rh factor (monkey) \\ 1943 Vitamin $\mathrm{K}^{*}$ (rat, dog, chicken, mouse) \\ 1956 Open heart surgery and cardiac \\ pacemakers (dog) \\ 1964 Regulation of cholesterol (rat) \\ 2002 Mechanisms of cell death* (worm) \\ * Nobel Prize awarded for the research
}

who was stolen, sold, and resold; that event prompted an unbelievable outcry in the public domain and demands for Congress to act. Congress did act and since 1966 a number of subsequent policies, acts, and regulations have been put in place to protect the animals that are so vital for our research.

CDC history goes back to 1946 when the agency was established to control malaria because it was an important disease globally that was killing American soldiers, and because of numerous military posts in the Southeast of the United States where malaria was indigenous. Hence, we started as a malaria agency and our first work with animals involved killing mosquitoes. CDC has been accredited by AAALAC since 1966 and we had our first IACUC established in 1985. Several years ago, we established our Animal Care and Use Program Office (ACUPO). CDC is grateful to AAALAC and its leadership for helping us and guiding us especially over the past decade in developing policies and animal care and use programs, one of them ACUPO, to make CDC's animal care and use program an exemplary one.

\section{Box 2 Selected discoveries related to infectious diseases}

(a) Development of vaccines

1796 Smallpox (cow)

1881 Anthrax (sheep)

1885 Rabies (dog, rabbit)

1933 Tetanus (horse)

1954 Polio* (mouse, monkey)

1968 Rubella (monkey)

* Nobel Prize awarded for the research

(b) Work recognized by the Nobel Prize

1905 Pathogenesis of tuberculosis (guinea pig, horse, rabbit)

1928 Pathogenesis of typhus (guinea pig, rat, mouse)

1945 Penicillin tested (mouse)

1984 Monoclonal antibodies developed (monkey)

1997 Prions discovered (hamster, mouse)

Sources: www.amprogress.org, www.fbresearch.org
CDC has about 200 active protocols and 200 principal investigators at four physical locations in Atlanta, Lawrenceville, Morgantown, and Fort Collins. Three key areas of research are infectious diseases, reagent production for the detection of infectious diseases, and a smaller emphasis on environmental health including nanotechnology. We have three IACUCs.

One of the things that resulted from the work with AAALAC is the structure that shows how critically important oversight of animal care and use is at the agency. The three key components of CDC's animal care and use program are the IACUCs, the Animal Care and Use Program, and the veterinary and animal support staff. All of this converges toward the CDC Institutional Official (IO), and I have had the honor of serving as CDC IO since 2004.

There are 88 species of animals in use at CDC (including field studies), very similar to the kinds of animals that $\mathrm{NIH}$ is using in its research. Ferrets are extremely popular at CDC in influenza transmission studies as they have a lot of commonalities with humans, including similar host receptors for the influenza viruses and very similar disease progression. The CDC's research on animals is a reflection of our broad area of research in infectious disease globally.

I want to share what it is that the research on animals globally and at CDC has done for the global community. I will focus on five diseases-HIV/AIDS, influenza, hepatitis, rabies, and malaria - and demonstrate some of the key scientific accomplishments over the past few decades. I have separated, in some way, contributions made by others and the key CDC contributions in certain areas. These are not my personal picks and choices; I have consulted with colleagues who work in these areas. I do apologize in advance if this becomes excessively scientific, but I am primarily a scientist and then an IO, and I thought that sprinkling in some heavy science would not hurt.

I will start with influenza. Even without the H1N1 influenza, between 3,000 and 49,000 people die every year of illnesses in the United States associated with complications of seasonal influenza (Thompson et al. 2010). Information collected on a weekly basis by CDC on circulating influenza strains and trends in influenza illness are key activities. Major contributions of research on animals for influenza were in the development of antivirals; specifically, neuraminidase (NA) inhibitors, which we know as zanamivir (Relenza) and oseltamivir (Tamiflu), drugs that inhibit NA activities. NA is an enzyme that allows propagation of the virus in the upper respiratory tract and the movement of the progeny virus, the multiplied virus units, from an infected cell (von Itzstein 2007). Those have been extremely potent and valuable drugs. Safety and efficacy testing on these drugs has been done on animals, making them really much better for people, putting them into clinical trials at the point when much is already known.

Specific CDC contributions fall into several areas. One had a lot of attention a couple years ago and that was the reconstruction and characterization of the 1918 pandemic virus (Tumpey et al. 2005). Work with the reconstructed 
1918 virus was conducted at and supported by CDC. The USDA, NIH, and the Armed Forces Institute of Pathology (AFIP) all provided support for many other aspects of this research. One can inquire as to why it is important to know what the virus of 1918 looked like. It is because we would like to see what it is that made it so deadly, and we would like to see whether those components, in its genetic makeup, can be found in new and emerging viruses. This was one of the most fundamental contributions of our agency in flu research.

In terms of vaccines, we continuously evaluate influenza strains that come in from all over the world and assist in selection of the vaccine candidates. We also do a great deal of preclinical evaluation and then participate in evaluation of novel vaccine candidates and drugs. Our key scientist in this area is Dr. Terry Tumpey, who is also a member of our IACUC. Obviously, we want to get the most prominent CDC researchers to serve on the IACUC. Ten years ago we had a difficult time with that, but with AAALAC support and overall agency support, scientists now consider it an honor to serve on our IACUC.

The second disease I want to focus on is HIV/AIDS. The number of people affected is staggering. In spite of so many years of dealing with it we do not have a vaccine. There is a lot of research going on, and a vaccine would be the most important prevention measure. However, there are a lot of other efforts that scientists are undertaking that I will share. There are over 33 million people living with HIV today. That is living with HIV, not having a death sentence with HIV. Every day, more than 1,000 children are newly infected with HIV worldwide. Two million people die of AIDS every year. As can be the case with other infectious diseases, the distribution of disease burden is not equal in the world and thus it is usually those in the developing world who are most affected. The prevalence in Africa is unbelievable-there are countries where over $30 \%$ of child-bearing women are infected.

The contributions in terms of animal research when it comes to HIV/AIDS have been from very basic research in understanding the pathogenesis as to why it is so challenging to develop a vaccine and other prevention measures. Unlike for polio, one cannot say in a simple sentence, "The ultimate outcome of research was the development of a vaccine." But one can certainly say that there has been so much progress with regard to anti-HIV medication that people can live longer and fuller lives with HIV these days.

Several contributions are considered critical. Work on nonhuman primates and monkeys has allowed for a better understanding of phylogenetic relationships among different HIV viruses and simian retroviruses, which has made it possible to develop simian models to advance HIV research using simian immunodeficiency virus (SIV). Many consider this one of the most fundamental contributions of animal research (Daniel et al. 1985; Letvin et al. 1983). Another major contribution has been development and refinement of a macaque monkey model, known as the repeated low-dose (RLD) model. This model has had a profound impact on
HIV prevention research, especially with regard to antiretroviral preexposure prophylaxis medications that are now considered to be a potentially important way to help people prevent HIV infection. Clinical trials are looking at different means and routes (e.g., oral or vaginal) of delivering antiretroviral preexposure prophylaxis medications to the site of initial HIV infection. These studies have immensely benefitted by the development and results obtained from the RLD model and the initial work using macaque monkeys.

What CDC does cover, in its own way and within its spectrum, includes a broad array of activities: from core work, such as model development, pharmacodyamic and pharmacokinetic studies, all the way to drug resistance and virus susceptibility studies. In addition, novel interventions and devices including pills, gels, cervical rings, and the like are being explored that have promise for preventing HIV infection in people.

The third disease I am focusing on is viral hepatitis. Again, it has an unbelievable global burden: 350 million people live with the hepatitis $\mathrm{B}$ virus (HBV) as a chronic infection, and 170 million are chronically infected with hepatitis C. In the United States, there are about 3,000 documented, reported cases of hepatitis $\mathrm{A}$, which translates to about 25,000 cases a year because reported and actual cases are not necessarily the same, depending on the kind of surveillance available (e.g., how reliable the reporting is). But there was a dramatic decline in the number of cases following the introduction of the hepatitis A vaccine. For acute hepatitis B there are about 4,000 new cases every year, with an estimated 38,000 in 2008. There was a decline in the number of cases after the introduction of the vaccines.

A key contribution to the discovery of the $\mathrm{HBV}$ vaccine, the main component being the HBV surface antigen, comes from the use of chimpanzees for vaccine efficacy studies. Most of the work for hepatitis is done on chimpanzees, and that is the only infectious disease for which research at CDC is conducted on chimpanzees. A study by McAleer and colleagues (1984), more than a quarter-century old, is the first example of a vaccine produced from recombinant cells that is effective against human viral infection. The CDC also contributed to the discovery of hepatitis $\mathrm{C}$ virus via its pioneering work on infectivity studies done on chimpanzees (Choo et al. 1989). One of the things we realized several years ago is that there comes a time when one must decide whether and how much to focus on the research on chimpanzees. We have narrowly limited this research and moved all six chimpanzees from the CDC campuses to New Iberia in Louisiana. We consider that a major accomplishment because we felt that in terms of social settings life would be much better for them there.

Rabies is a disease that is frequently forgotten because we do not hear about it often and we do not think about dying from it. However, it occurs in more than 150 countries worldwide and over 50,000 people die every year, many of them children. Every year, 15 million people get rabies postexposure prophylaxis. Unfortunately, once a person has been exposed, without such prophylaxis there is only one way to 
go: to die. Postexposure prophylaxis is extremely important and it is again the animal work that has enabled us to understand its value. It is fascinating to think that something we still consider a major contribution when it comes to animal research for rabies goes back 60 years (Koprowski et al. 1950). Postexposure prophylaxis using a guinea pig and hamster model set the stage for the recommendations we use today (i.e., to use the serum and a vaccine), and this is what the Advisory Committee on Immunization Practices at CDC still recommends. This major contribution saves an extraordinary number of lives.

One CDC contribution that I am very proud of is that the research on animals can be helpful to the animals even though it is primarily intended for humans. Specifically, that is the development of an oral vaccine for animals against rabies, the first experimental use of oral vaccination (Baer et al. 1971). In Western Europe and the United States there is a substantial decrease in the number of cases among animals. Why is this important? It is important because this shows that we can prevent an infection in animals without slaughtering them. When I talk to my colleagues who study rabiesevery September there is a global conference on rabies at $\mathrm{CDC}$ - they say that the pictures showing how cruelly animals are treated in some parts of the world because of prevention of rabies are just too horrible to see. To have a vaccine that saves animals' lives and prevents drastic measures is an unbelievable success, in addition to the 15 million people that receive prophylaxis whose lives are also saved, thanks to the laboratory animals.

And the last disease that I will briefly mention is malaria. Today, 3 billion people in the world live in areas where they are susceptible to malaria transmission-in over 100 countriesand it is estimated that about 1 million people die every year from malaria. Most of those deaths occur in Africa. Malaria is the fifth leading cause of death from infectious disease in the world and the second in Africa. Cases in the United States are, as a rule, imported. Key contributions of animal research for malaria go fairly far back; they are in the development of drugs against malaria (Davidson et al. 1976). Understanding the pathogenesis of malaria through the use of animal models facilitates work on the development of vaccines and other preventive measures. A nonhuman primate model using monkey malaria was key in this study for developing the drugs active against relapsing forms of malaria, which is a major problem for malaria prevention and control. A key CDC contribution has been the development of many different models in monkeys for testing of different types of vaccines and drugs against Plasmodium falciparum and $P$. vivax. Models using monkey malaria parasites have also been developed that can serve as substitutes for various drug and vaccine studies where human malaria parasites and New World monkey hosts are not available (Collins et al. 2006).

I would like to end with a couple of general comments. If there is one thing to take from this talk, it is that there are hundreds of millions of people whose lives have been affected in a positive way from research where animals are critical. At the same time, animals used in research do not volunteer and do not give their consent, and we should be immensely thankful and appreciative of the sacrifices they make. We have to be just as grateful to those of you who have devoted your entire careers to animal welfare. There's an old saying that it's not what you do, it's how you make people feel while you're doing it. Recollections of such colleagues about their own experiences exemplified why their efforts were successful. It was because they made scientists feel good and ethical knowing that their research can not only be done but done with dignity and respect for the animal.

It was an honor to be a part of this conference with all of you and those who made it possible for all these accomplishments to take place and for millions of lives to be saved.

\section{Acknowledgments}

The author acknowledges contributions from the following CDC colleagues to these remarks: Ron A. Otten, PhD, Director, and Tim Barrett, PhD, Manager, ACUPO, both in the Office of Scientific Integrity, Office of the Associate Director for Science; Neelam D. Ghiya, MPH, Office of the Associate Director for Science.

\section{References}

Baer GM, Abelseth MK, Debbie JG. 1971. Oral vaccination of foxes against rabies. Am J Epidemiol 93:487-490.

Choo QL, Kuo G, Weiner AJ, Overby LR, Bradley DW, Houghton M. 1989. Isolation of a cDNA clone derived from a blood-borne non-A, non-B viral hepatitis genome. Science 244:359-362.

Collins WE, Sullivan JS, Williams A, Nace D, Williams T, Galland GG, Barnwell JW. 2006. Aotus nancymaae as a potential model for the testing of anti-sporozoite and liver stage vaccines against Plasmodium falciparum. Am J Trop Med Hyg 74:422-424.

Daniel MD, Letvin NL, King NW, Kannagi M, Sehgal PK, Hunt RD, Kanki PJ, Essex M, Desrosiers RC. 1985. Isolation of T-cell tropic HTLV-IIIlike retrovirus from macaques. Science 228:1201-1204.

Davidson DE Jr, Johnsen DO, Tanticharoenyos P, Hickman RL, Kinnamon KE. 1976. Evaluating new antimalarial drugs against trophozoite induced Plasmodium cynomolgi malaria in rhesus monkeys. Am J Trop Med Hyg 25:26-33.

Koprowski H, Van Der Scheer J, Black J. 1950. Use of hyperimmune anti-rabies serum concentrates in experimental rabies. Am J Med 8: 412-420.

Letvin NL, Eaton KA, Aldrich WR, Sehgal PK, Blake BJ, Schlossman SF, King NW, Hunt RD. 1983. Acquired immunodeficiency syndrome in a colony of macaque monkeys. Proc Nat Acad Sci U S A 80:2718-2722.

McAleer WJ, Buynak EB, Maigetter RZ, Wampler DE, Miller WJ, Hilleman MR. 1984. Human hepatitis B vaccine from recombinant yeast. Nature 307:178-180.

Thompson MG, Shay DK, Zhou H, Bridges CB, Cheng PY, Burns E, Bresee JS, Cox NJ; Influenza Division, National Center for Immunization and Respiratory Diseases, CDC. 2010. Estimates of deaths associated with seasonal influenza: United States, 1976-2007. MMWR 59:1057-1062.

Tumpey TM, Basler CF, Aguilar PV, Zeng H, Solórzano A, Swayne DE, Cox NJ, Katz JM, Taubenberger JK, Palese P, García-Sastre A. 2005. Characterization of the reconstructed 1918 Spanish influenza pandemic virus. Science 310:77-80.

von Itzstein M. 2007. The war against influenza: Discovery and development of sialidase inhibitors. Nat Rev Drug Discov 6:967-974. 


\title{
Use of Animals in the Development of Medicines and Devices for Humans and Animals
}

\author{
David Jacobson-Kram
}

t's a pleasure to be here this morning. In fact, it's a pleasure to be this morning. A couple of weeks ago, on my way up to my roof, I fell off a ladder-and I thought about all the different drugs and devices that were used in treating me and the contributions of animal welfare in all those products. It's an amazing number of products that I personally benefitted from just in the last few weeks.

I'll tell you a little bit about FDA. We have six regulatory centers:

- the Center for Drug Evaluation and Research, where I work, regulates all drug products;

- the Center for Biologics regulates biological products such as vaccines, blood, cellular and blood therapy, allergenics, and tissues;

- the Center for Devices and Radiological Health regulates medical devices and radiation-emitting electronic products;

- the Center for Veterinary Medicine looks at food additives and drugs that will be given to animals, including those from which human foods are derived, as well as food additives and drugs for companion animals, pets;

- the Center for Food Safety and Applied Nutrition looks after food-borne illnesses, nutrition, dietary supplements, all food products except meat, poultry, and some egg products; and

- the most recently inaugurated, the Center for Tobacco Research; its mission is public education and reducing tobacco-related diseases.

There's also the National Center for Toxicological Research in Arkansas, which is not a regulatory center but focused primarily on toxicological research.

To give you a sense of our mission, imagine various pharmaceutical companies all over the world with the capacity to synthesize hundreds of thousands of new molecular entities every day. The advances in medicinal chemistry have been incredible.

Drug candidates currently being synthesized are generally highly complex. A new chemical entity might be designed to bind at the catalytic site of an enzyme or perhaps to a membrane receptor. The next step in development is to give it to humans in a phase I study that examines pharmacokinetics and tolerability. The goal is to determine how human beings react after they're exposed to this new chemical entity. It's important to remember that phase I trials are generally performed in healthy volunteers. There are exceptionsfor example, oncology drugs are typically tested in patients with cancer because they tend to be fairly toxic_- but the vast majority of new drugs are tested in healthy volunteers. Unlike taking an approved drug or participating in a latestage clinical trial, there's no risk-benefit analysis because the only thing these participants have to gain is monetary compensation. Because this is human experimentation on a population that really has nothing to gain from exposure to this drug, the bar for safety is extremely high.

What do we need to know about a new chemical entity in order to safely perform a phase I study? We need to know how much drug should be given to the study participants in the first dose. You have a new chemical entity, you don't know very much about it, and you have this healthy human population. How much are you going to give them the first time? What type of toxicities might be elicited by this chemical entity? What parameters need to be monitored very closely in this clinical trial? For example, if liver toxicity is the one you're most worried about, that's the one you're going to focus on. If the participants tolerate the drug, what constitutes a stopping dose? If they didn't get nauseous, they didn't get a headache, didn't have any adverse responses, when do you stop?

There's a set of preclinical studies prior to a human phase I trial. The candidate drug is tested in two species, a rodent and a nonrodent. For small molecules, that's generally a rat and a dog and for biologic products the nonrodent is generally a nonhuman primate. We try to use the same route of exposure that's going to be used in the clinical trial. So if the intended route in the clinical trial is oral, that's the route that's going to be used in the toxicology studies.

Here are some examples of endpoints that would be monitored during the in-life phase. We would look at clinical signs - animals have very characteristic behavior patterns and if you're used to dealing with the animals and they display abnormal kinds of activities those things are easily noted by experienced people. We monitor the weight gain of the animals, their food consumption, and then, after the animals have been euthanized, we look at parameters like hematology and clinical chemistry and gross pathology and histopathology.

Once we have these data in hand, what do we do with them? The first thing we do is use that information to select a safe starting dose. How much of this new chemical entity can we safely give to the first human being who's ever going 
to be exposed to it? We want to identify the organs of toxicities. We want to point out what organ systems need to be monitored very closely because this chemical was found to induce toxicities in those organ systems. We want to identify a safe stopping dose, so even if the trial participants are not demonstrating any adverse effects, at some point we want to say you shouldn't give any more of this drug even if the participants aren't experiencing any adverse effects.

Another important parameter that we want to measure is reversibility of a toxicological response. For example, let's say we see nephrotoxicity: we want to understand whether once we stop giving this drug the kidneys recover; sometimes they do, sometimes they continue to decline, and that's a very important distinction to understand. Once this information has been produced, it's included in the informed consent that the participant signs and also in the investigator's brochure.

How do we go about calculating a safe starting dose? If you go to the FDA website (www.fda.gov), you can find guidance, "Estimating the Maximum Safe Starting Dose in Initial Clinical Trials for Therapeutics in Adult Healthy Volunteers," a very detailed step-by-step cookbook kind of instruction that allows you to calculate what a reasonable safe starting dose would be. The really pivotal information here is the NOAEL, the no observed adverse effect level, in the more sensitive species. If you've tested in two species, we want to use the NOAEL from the species that is more sensitive, and then that is converted to a human-equivalent dose. It gives you all the formulas you need to do that and then we add a safety factor. The size of that safety factor hinges on things like which organ systems are at risk, is the toxicity reversible, and so on. The more risk that's associated with it, the greater the safety factor; the more benign the toxicities, the smaller the safety factor can be.

So these are the types of preclinical and nonclinical tests. Pharmacology and mechanistic studies are preregulatory. They are done by the pharmaceutical companies to convince themselves that their drug will have efficacy once they take it to humans. All safety testing is designed for regulatory submission and is performed according to good laboratory practices (GLPs). Such studies include safety pharmacology (looking at CNS effects, respiratory effects, or toxicity to the cardiovascular system), general toxicology studies, genetic toxicology, pharmacokinetics, ADME studies (absorption, distribution, metabolism, and excretion), reproductive toxicology, and carcinogenicity.

Why do we feel that these are important studies? In addition to enabling first-in-human clinical trials, animal studies are used to assess potential toxicities that cannot be studied in clinical trials. For example, (1) teratogenicity: you want to know if the drug has the ability to induce birth defects, but one wouldn't deliberately expose pregnant women to a drug to find out if it induces birth defects, obviously that would be highly unethical; (2) carcinogenicity: you want to know if the drug is potentially carcinogenic, but the long latency period and the insensitivity of epidemiological studies preclude the identification of this adverse effect; and (3) long-term toxicities - a clinical trial, even a long one, might go on 2 or 3 years, but after approval a drug might be taken for many years, in some cases for a lifetime, yet it's been studied in people for only a very small fraction of a total lifespan. Chronic studies in animals can provide a sense of what the drug's long-term toxicities might be.

I'm sure everyone here is familiar with thalidomide. This was a horrific incident and caused very severe limb abnormalities. This was a nonmonitorable toxicity. The drug was prescribed to pregnant women for nausea and insomnia. It resulted in 10,000 births with severe limb malformations. The link between exposure and adverse effects was possible because of the potency of the drug and the relatively short time period between exposure and the manifestation of the adverse effects. The association wasn't that difficult to make because the women who gave birth to these children had a prescription for this particular drug and the malformations were very severe. An example for carcinogenesis that I'm sure you're all familiar with [is] diethylstilbestrol (DES). It was prescribed to pregnant women to maintain pregnancies. Ironically, the drug was not effective for this indication but what it did do was increase the risk to 1 in 1,000 for clearcell adenomas of the vagina and cervix in female offspring. So it didn't induce cancer in the women who took it but rather in their daughters. The link between exposure and risk was possible because of the rarity of the tumor type-it is a highly unusual kind of cancer. If exposure increased the risk for a common cancer, this association would have been much more difficult to make.

Safety versus efficacy. Most animal studies are used to ensure the safety of clinical trial participants and of patients that are going to consume the drug after it's been approved. Under some circumstances animal models can also be used for demonstrating drug efficacy. This is primarily in the area of counterterrorism products.

We have another guidance that you can see on our website. It's called the Animal Rule and presents essential elements needed to address efficacy. It states that in selected circumstances, when it is neither ethical or feasible to conduct human efficacy studies, FDA may grant marketing approval based on adequate and well-controlled animal studies when the results of those studies establish that the drug or biological product is reasonably likely to produce clinical benefit in humans and demonstration of the product's safety in humans is still necessary. You still have to do a clinical trial to show safety but obviously you won't infect a healthy volunteer with something like anthrax.

Here are some examples of drugs that were approved under the Animal Rule. We had an efficacy supplement for Cipro that was approved for postexposure management of inhalational anthrax (this was a result of the terrorism incident where anthrax was put into envelopes and mailed). There's a Cyanokit for treatment of known or suspected cyanide poisoning. And there's a drug that's used to increase survival after exposure to the nerve agent Soman.

What have we at FDA done to reduce animal use and what are our plans going forward? We are a member of the Interna- 
tional Conference on Harmonization (ICH). This is a worldwide consortium that meets three times a year-once in Europe, once in the United States, and once in Japan - and drafts guidelines for drug development in the areas of efficacy, safety, and quality. If one adheres to the guidelines in developing drugs, the studies performed will be acceptable in all three geographical locations. This has helped reduce redundancy in testing. It used to be that the United States had its own guidance, Japan had theirs, and Europe had theirs, and one would have to comply with everyone's guidelines in order to get a drug approved. This resulted in a lot of testing that was redundant. The $\mathrm{ICH}$ has eliminated a lot of that. A major guiding principle in developing new safety guidelines is reduction of animal use.

Some examples where we've been successful are in carcinogenicity testing. ICH accepts the results of shorter-term assays-6-month studies in transgenic mice-in lieu of a 2-year mouse study. This has reduced the use of mice by half and that's quite significant because carcinogenicity studies use very large numbers of animals. On acute toxicity testing, ICH clearly states that acute single-dose studies are unnecessary because the toxicity of drugs is studied so closely in so many different kinds of assays, we feel that single-dose studies are redundant and unnecessary. In genetic toxicology recently we've eliminated the need for positive controls with each in vivo study.

What about the -omics revolution we hear so much about-how can this help in the assessment for carcinogenicity? It can shorten the time required to determine whether a drug or chemical is potentially carcinogenic. It can lower the cost of testing, which will allow more compounds to be tested. It will improve extrapolation of animal data to humans. It will improve extrapolation from experimental high doses to human exposure levels. It will reduce animal use. But perhaps most importantly, it will provide insights into mechanisms of action.

Very often when one performs a carcinogenicity study with 2 years in-life and another year postlife one finds that the drug induced tumors. Often one does not understand why it did. How do you develop the next generation drug to be noncarcinogenic if you don't understand the mechanism by which this drug induced tumors in the first place? We're part of a research consortium called the Critical Path and we have different areas that we focus on and one of them is a Carcinogenicity Working Group, of which I'm a member. Our short-term objectives are to identify and evaluate the utility of genomic biomarkers to provide an early prediction of carcinogenicity, especially by nongenotoxic compounds, and to develop an assay platform and test protocol to enable the early prediction and mechanistic assessment of carcinogens. So we can take animals, expose them to a new test compound and study changes in gene expression, which genes are induced and which genes are suppressed. We might be able to develop a signature or battery, so whenever we see a particular change in gene expression it would portend a positive carcinogenicity study. We've made progress in this area.
Our long-term objective is to determine whether genomic data combined with other subchronic or chronic toxicity endpoints can reduce reliance on lifetime rodent bioassays.

Here's an example of how this can be done. A few years back, a group looked at 100 paradigm compounds to develop a signature for nongenotoxic carcinogens. They gave rats a single high dose, a significant fraction of a published LD50, of each chemical and the livers were removed 24 hours after dosing. A training set, consisting of 24 nongenotoxic carcinogens and 28 noncarcinogens, was used to identify a sixgene signature that identified nongenotoxic carcinogens with a remarkable accuracy of $88 \%$. By using the training set, they found a particular set of six genes whose altered expression is indicative of a nongenotoxic carcinogen. This was the case whether the liver was or was not the ultimate target organ of carcinogenesis. This work is the result of an interlaboratory evaluation of genetic signatures for predicting carcinogenicity in the rat. These efforts demonstrated that, with a large number of compounds, expression arrays can inform us about potential carcinogenicity with very high sensitivity and specificity. We're truly making progress in this area.

Finally, I want to mention a memorandum of understanding (MOU) that FDA signed quite recently-High-Throughput Screening, Toxicity Pathway Profiling, and Biological Interpretation of Findings - with the National Toxicology Program, NIH Chemical Genomics Center, and EPA. The goal of the MOU is to collaborate in the development, validation, and translation of new and innovative test methods that characterize key steps in toxicity pathways. What we want to be able to do is to understand the pathways between exposure to a toxin and the manifestation of pathology. Between exposing a cell in culture or an animal to a particular toxic agent and the pathology that you see, there's going to be a network of pathways that cause that pathology to express. A central component of this MOU is the exploration of tests using phylogenetically lower animal species, for example fish and worms, as well as high-throughput whole-genome analytical methods, to evaluate mechanisms of toxicity. The FDA has a lot of toxicity information in human beings as well as animal models. The goal is ultimately to be able to study cells in culture to determine whether toxicity pathways are being activated. That information will indicate what pathologies we might anticipate in human populations, and hopefully by not exposing people to those compounds those toxicities won't occur.

In conclusion, for the time being animal testing is essential to ensure the development of safe drugs, medical devices, food additives, and biological products for humans and animals. FDA and other federal agencies are aggressively moving toward reducing animal use through programs like the International Conference on Harmonization. It is my personal belief that through programs like Tox 21 we will ultimately eliminate the need for animal studies, at least in the development of pharmaceuticals. 


\section{Perspectives of PHS Funding Agencies: Discussion}

Questioner: Yesterday we heard a little about changes in housing and care of laboratory animals. This morning I'm hoping each of you panelists can answer a question about the sources of animals used in research, some of which you described today. Please let us know your agency's position on the use of animals obtained (1) from convicted felons, given that one of the primary primate importers is a convicted felon from smuggling endangered primates, and (2) your agency's position on the use of Class B dog and cat dealers?

Dr. Rockey: I can start on our sources of animals. Of course we have to fall under all of our guidelines for the appropriate receipt of animals. I'm not sure about the case you're talking about with a convicted felon, but of course we are abiding by all of our policies. In the case of Class B dealers, NIH, as many of you know, has been looking at the use of dogs and cats obtained from such dealers. We had some instructions from Congress on use, and also as you know there was a National Research Council study on the use of Class B dealers. We're currently revisiting our policy on the sources of dogs and cats used in our research.

Dr. Popovic: I can give basically the same answer as Dr. Rockey. I will be happy to send follow-up information to confirm that we are not dealing with sources of convicted felons and similar. But I am not aware of the specific case mentioned.

Dr. Jacobson-Kram: I'm not that familiar with that case either. But all the studies that are done to be submitted as part of a new drug application are GLP studies so they're performed in GLP facilities, they are inspected by AAALAC, and also we have our own division that goes around to contract labs and pharmaceutical labs to make sure that there is compliance with animal care and use.

Questioner: I have two questions for Dr. Rockey. As far as NIH grants and what direction funding would be most impactful, is there a mechanism for critiquing certain areas of research? I've seen arguments for hepatitis $\mathrm{C}$ using chimpanzees but a lot of the citations used have been 10, 20 years old [whereas] the culture, in vitro tests, have been developed in very recent years. Is there a way of critiquing what's going on and where the funding should be going instead of looking grant by grant as its own little world?

Dr. Rockey: All of our applications go through a rigorous peer review and [have to include] justification for the use of animals and for the model system and why that system is most appropriate. In the case of hepatitis $\mathrm{C}$, the chimpanzee is our only current model. When the study session takes a look at a grant, they're looking at the grant itself and the specific project, [and] they're looking at that project in the whole area of science, so they aren't just looking at it as an individual, they're looking at it in a more holistic approach.
Questioner: So there are determinations made about where the funding would best be going? For example, if there's in vitro [research] that's really exciting and new, there's more funding invested in that kind of area, or...?

Dr. Rockey: Yes. It's based on the merits of the application. If there is an alternative, particularly in the case of hepatitis $\mathrm{C}$, that would be viewed in the context of what they're doing and how appropriate that science is.

Questioner: I'm Bill Stokes, NIH National Institute of Environmental Health Sciences and National Toxicology Program, Executive Director for the Interagency Coordinating Committee on the Validation of Alternative Methods, which is operated out of our institute. This is more of a comment. We just had an international workshop on alternative methods to reduce, refine, or replace animals in vaccine potency and safety testing and all of you agencies participated in it—organizing, speaking at, and otherwise taking part in it - and I just want to make sure that folks here are aware of that. There will be a proceedings with information about the outcomes in terms of recommendations for making progress in this area because, whether we like it or not, vaccine potency testing for some vaccines still involves a large number of animals and accounts for a significant number of the animals and colony listings on the USDA report form. I think this workshop made great progress in identifying new advances in science and technology that can be applied to come up with better ways to assess potency and safety as well as to benefit animal welfare by reducing and replacing animals, as [has been done] for many of the vaccines that have been developed lately.

Questioner: I want to direct this initially to Dr. JacobsonKram. It's very laudable to try to use in vitro systems to study compounds, but especially with cell lines these cells change their properties and culture and are no longer in a complex intercellular organization as they are in living organisms. It should be recognized that jumping directly from culture to human studies does create a risk to the human population by eliminating some of the animal experimentation that people need to focus on and not just thinking that in vitro studies can replace everything.

Dr. Jacobsen-Kram: I don't think we're at that point yet and in vitro systems have limitations as you point out, especially in the area of providing metabolic activation. A lot of chemicals are converted to their toxin form by the liver in an effort to make them more water soluble and eliminate them from the body.... [Others] of our systems don't have this ability, they don't have the cytochrome 450 enzyme, and that's a limitation, but people are working on that. We're not at the point yet where, for example, we could replace much of the animal testing with in vitro results but 
it's my hope that, by understanding toxicity pathways and making the connection of those pathways to human pathology, we will be able to do that in the not too distant future. The key understanding is the toxicity pathway, so that when we see that a chemical triggers a particular pathway we'll say, "That's associated with liver toxicity or nephrotoxicity, we don't want to move that compound forward because there are a lot of [others] that have the same potential efficacy but don't carry that toxic liability." I think initially it's going to be very useful in screening chemical libraries where you have thousands of compounds. You could quickly get rid of $90 \%$ of them because they're found to trigger certain toxicity pathways that you wouldn't want to [encounter] clinically. 


\section{Animal Models and Basic Science—Bench to Bedside: Session Introduction}

\section{Richard Nakamura}

'm Scientific Director at the NIH National Institute of Mental Health (NIMH). I came to NIH from my PhD work at the State University of New York at Stony Brook. I began conducting, essentially, information processing research in primates. I worked on that for about 15 years and gradually converted to more administrative and program positions at NIMH, where I started over 30 years ago. I'm going to talk to you about some of the observations over that period of time on animal models.

Our second speaker will be Dr. Linda Cendales, an assistant professor of surgery in the Division of Plastic and Reconstructive Surgery and Division of Transplantation, Department of Surgery at Emory University School of Medicine. She is also Director of Vascularized Composite
Allotransplantation and the Laboratory of Microsurgery at the Emory Transplant Center. She has been formally trained in hand microsurgery and transplant surgery.

The last speaker will be Dr. Michael Kurilla, Director of the Office of Biodefense Research Affairs and Associate Director for Biodefense Product Development for the NIH National Institute of Allergy and Infectious Diseases here in Bethesda. His primary role is to provide overall Institute coordination for product development for medical countermeasures against bioterror threats. He received his undergraduate degree in chemistry from California Institute of Technology, earned his PhD-MD from Duke, and did postdocs at Harvard. He was an assistant professor at the University of Virginia and worked at three major pharmaceutical companies before joining the NIAID.

\section{Animal Models and Basic Science-Bench to Bedside}

\section{Richard Nakamura}

am going to talk about animal models for mental health and biology. Mental illnesses have a tremendous disease burden. Harvard University, the World Bank, and the World Health Organization did an analysis of disease burden by illness, using disability-adjusted life year, which balances chronic diseases with diseases that are acute and cause death. They looked at the top ten disease conditions that cause disease burden around the world in 2000 across all ages. [They found that] lower respiratory infections had the greatest disease burden, perinatal conditions the second most, HIV/ AIDS third, and unipolar depressive disorders fourth. [The remaining top ten were] diarrheal diseases, ischemic heart disease, cerebral vascular disease, road traffic accidents, malaria, and tuberculosis.

They did a number of different kinds of analyses, one of which was the analysis of disease burden in 15- to 44-yearolds. They [wanted to determine] what caused the most disease burden in the segment of the population around the world in which society had made a critical investment in development - through parental investment and educationand was expecting some yield on that investment. I suspect that one of the key reasons they picked this segment was not only what was affecting the population expected to be most productive but also what was the effect of HIV/AIDS on the world burden. Clearly HIV/AIDS was by far the most significant disease burden for this population. We were very interested in the fact that unipolar depressive disorders and many behavioral conditions suddenly emerged as important disease burdens. Road traffic accidents, alcohol use disorders, self-inflicted injuries or suicide, schizophrenia, bipolar disorder, and violence also ended up in the top ten conditions causing disease burden for this population worldwide.

For the same age group in the United States, Canada, and Western Europe, where contagious illnesses became less significant, unipolar depressive disorder and a number of other mental and behavioral conditions became the diseases with the greatest burden. Depression was number one, alcohol use number two, road traffic accidents number three, drug use number four, suicide number five, then bipolar disorder, migraine, schizophrenia, hearing loss, and, last of the top ten, HIV/AIDS. So there's a huge shift as you move from underdeveloped to developed countries, looking at the age 
[group] of people expected to be highly productive in society. Mental and behavioral disorders, chronic disabling conditions, have the highest disease burden.

Mental disorders also have significant mortality, accounting for approximately 30,000 suicides per year in the United States. The Institute of Medicine concluded that $90 \%$ of these suicides related to mental illness. For context, there were 18,000 homicides, 20,000 AIDS deaths, and only three forms of cancer [claimed more than] 30,000. It is important to think about what causes enough pain and suffering for an individual to decide to take his or her life. This [suicide rate] suggests that mental illnesses are almost unique in their capacity to cause that level of pain and suffering.

Another part of the public health impact is the early mortality suffered by those with major mental illnesses. One survey across states, [using] data of outpatient and inpatient clients diagnosed with major mental illnesses, showed that on average they died 25 years younger than the life expectancy in the United States. So there's a huge early mortality associated with this illness. This is the context in which we're trying to do research that will change this balance and reduce the burden of mental illnesses.

I'll point out that over and over again we see that all life on earth has a common origin. Figure 1 shows a version of the tree of life, with the eukaryotes, the tiny branch that includes humans, in the top group. This illustration shows one possible theory about the root of life about 4 billion years ago. The eukaryotes are thought to have started around 2 billion years ago and, at the last end [on the far left, among the opisthokonts], is animal life, with humans emerging at the very tail of a long string in which all of these elements and all of these parts come back to common origins. This means that there is a strong genetic homology, particularly among the eukaryotes, from yeast to worms to flies to fish to birds and mice to nonhuman primates and humans (see Box 1). This tree of life-with its constancy of mechanisms of inheritance, biochemistry, and systems biology —is the reason animal models are so useful in understanding the biology of human beings.

So far in the human genome project over 180 species have been completely sequenced, and this number is

\section{Figure 1 All life on earth has a common origin}

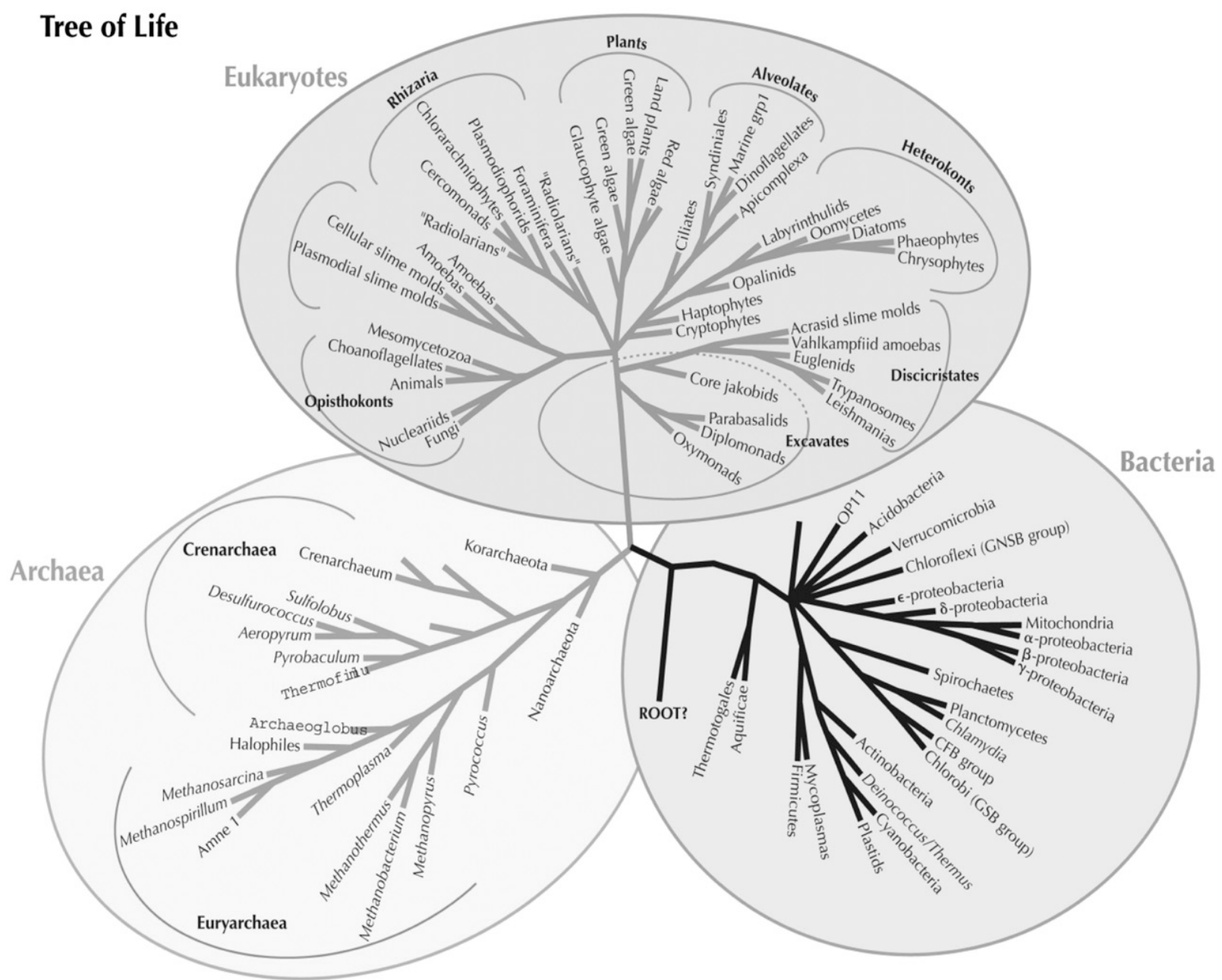




\section{Box 1 Why are animals useful in research?}

- Genetic homology from yeast to worms, flies, fish, birds, mice, to nonhuman primates and humansover 180 species sequenced

- Conserved genes most important

- Ability to manipulate genome and environment

- Common systems (circulatory, metabolism, sensory, motor, memory, emotion)

- Species differences can be useful

increasing very quickly. Those species all reaffirm that there's a common genetic background-the same genes are used over and over again with only modest changes across species. It's very clear now that there are many conserved genes and that they play critical roles in the development of our bodies and our brains. The ability to manipulate the genome and the environment for animals plays a critical role in our understanding of how this biology works. There are common systems across these organisms... of circulatory, metabolic, sensory, motor [systems], and even things like memory and emotion.

Even species differences can be very useful. As we've heard, some species can catch or have certain diseases and others cannot. Understanding the reasons for those differences can help us understand pathogenicity and disease alleviation in humans. More fundamentally, we can also learn about other human characteristics such as the importance of vocalization or of social systems. You'll hear more about those from speakers later today.

There has been a dramatic change in our understanding of behavior, both human and animal, including the way we think, and this has amounted to a modern integration of ideas that were long debated as being separate-like the ideas of brain and behavior, mind and brain, nature and nurture, structure and function, genes and the environment. We heard..."or" or "versus" between these words. But today we know that these are all concepts that are two sides of the same coin-you cannot talk of one without the other. Today we know that the gene guides the general structure of the brain throughout our lives and that the brain is the substrate for thoughts and behavior. The physical structure of our brain changes under the influence of behavior and the environment. So there is a two-way interaction, which means that brain and behavior are sides of the same coin.

It's hard to imagine that just 11 years ago a publication came out on neurogenesis in the adult human. This idea came to the attention of the larger neuroscience community from the lab of Fernando Nottebohm, who was looking at songbirds and [wondered] how they learned new repertoires every year. In the process he discovered that in their brain new cells were being born every year, [and that] this occurred seasonally. Initially, those who worked in human biology said, "We all know that there are no new neurons after adulthood in humans. This is something unique to birds and irrelevant for mammals and for human beings." Then 11 years ago, in a heroic study, neurogenesis was demonstrated in human cancer patients at the end of their life who allowed themselves to be injected with a substance that would stain new neurons (Eriksson et al. 1999). This showed that even through the eighth decade of life, in people who were dying - expected to die shortly from cancer-new neurons were growing in their brains. This was a momentous discovery. It changed the way we thought about the brain and the structure of the brain, as not fixed but in constant action and movement and constantly changing.

We learned also that other things could change neurogenesis. Physical activity could increase it (van Praag et al. 1999) and mental events like stress could reduce it (Gould 2000). In addition, certain medications increase or reduce neurogenesis.

The environment and behavior also change the structure of the brain through changing gene activity. Michael Meany did a tremendous study showing that there was epigenetic programming by maternal behavior (Weaver et al. 2004): the way rat mothers interact with their pups actually changed the way genes were structured, methylating certain parts of genes so that maternal licking and grooming changed the brain-changed genes-permanently by changing their activity (Meaney and Szyf 2005). This [change] could make subsequent generations of rat and mouse mothers high licking and grooming (Sapolsky 2004). We also found that environmental enrichment also changes the brain in similar directions. It is believed that this is the result of changing gene activity (Bredy et al. 2004).

At NIMH, we have a very difficult problem: to figure out very complex disorders, disorders that essentially were given to psychiatry because no one could figure out what caused them. These are devastating disorders that tremendously affect people's health, happiness, and productivity. But the question has remained: How do we get to the bottom of these disorders? Are they biological? Are they purely in one's head? We no longer believe the latter but [rather] that there's a biological interaction with everything. So the Institute started a program to try and work from genes to cells at different levels of organizationfrom cells to circuits to whole brain to individuals and, finally, to society. We have studies going on at all levels. We have long known that from genes you can go to a whole organism. What has become much clearer is that the organism, the environment of the organism - and society itselfchanges the way genes are interpreted, the way the brain is constructed. Working out this complex circuit in understanding how disorders-such as depression, schizophrenia, and bipolar illnesses-come about is critically important. Going through these levels, you ultimately get to a disordered behavior like schizophrenia, or autism, or mood disorders. And there are vulnerability genes that create vulnerability to the disorder. We also know that the environment and the disease conditions themselves can affect the structure and function of the brain. Working through this interaction is very important and complex. 


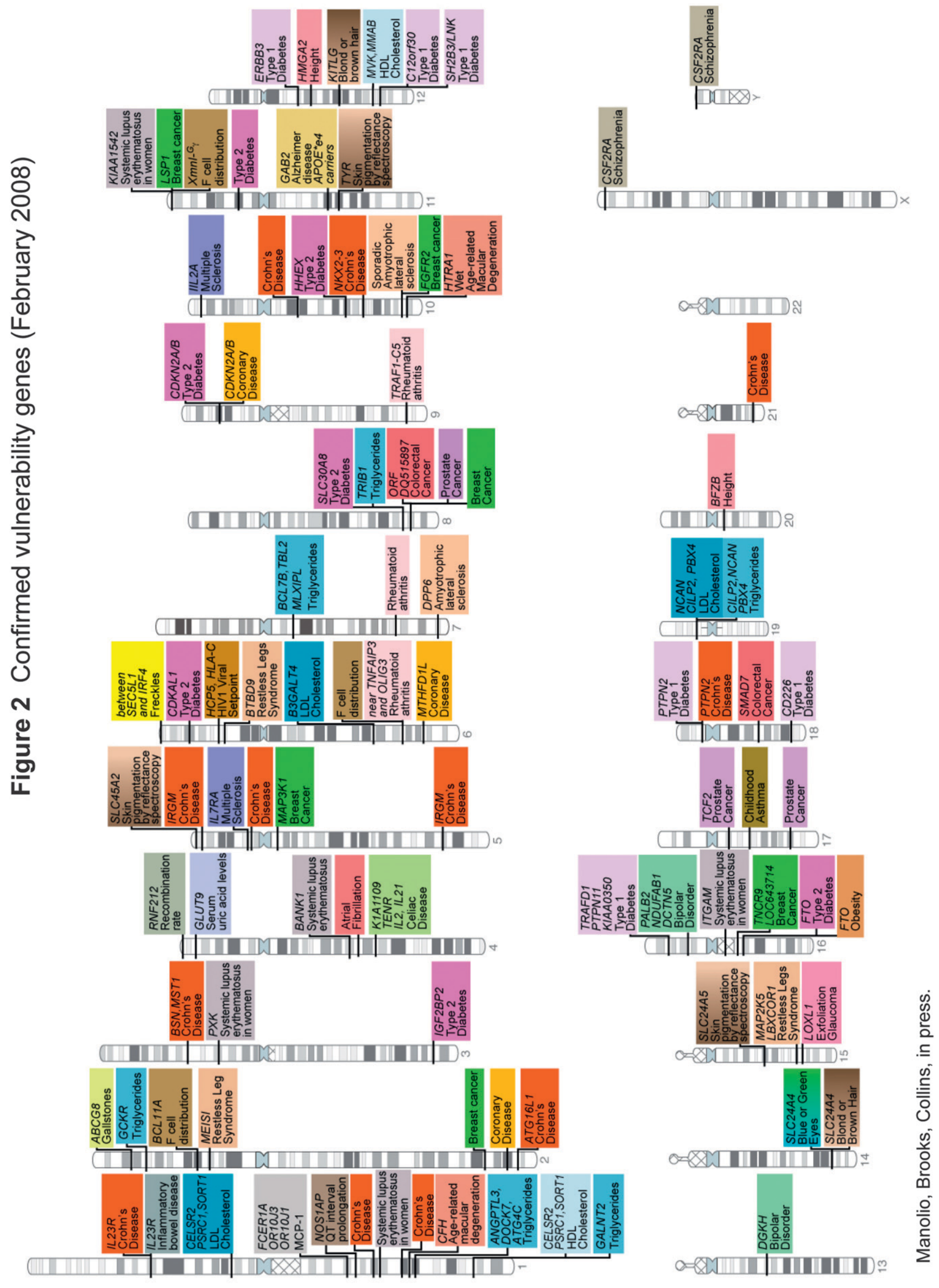


Just a decade ago we decoded the human genome, and very quickly thereafter genomewide association studies to relate the organization of that genome to vulnerability genes that may cause disease started producing fruit. An early success was to look for genes causing macular degeneration, which completely changed our view of the causes of this disease. And in rapid succession more and more vulnerability genes have been discovered. The illustration in Figure 2 was developed 2 years ago; if you see Francis Collins giving talks today, this graphic is completely covered with confirmed vulnerability genes. Studies have started to produce a first-level understanding of vulnerability genes associated with schizophrenia, bipolar disorder, and particularly autism, three disorders that are highly heritable. Something that has come out of an analysis of schizophrenia and bipolar illness is that there's a big overlap. Having a vulnerability to one increases your vulnerability to the other even though they don't coexist very much.

We started to find genes that code for personality factors, such as working memory, emotion regulation, and the like. The genes associated with working memory are critical for schizophrenia and those with emotion more important for bipolar illness (Meyer-Lindenberg and Weinberger 2006). But changes in these genes occur in both disorders. This has led to a proposal for how we can come up with new medications for such disorders.

Mark Bear (2008) pointed out that you can start with a developmental disorder in humans and use that disorder in large populations to generate gene discovery. From there you can look at the effect of changes in genes in mouse, fly, or even cell models. You can look at how those genes interact in the brain to identify cellular brain pathology. And from there one can identify molecular targets and then try to develop small molecule therapies and finally seek to treat the disorder. Bear (2008) has followed his own formula and developed the mGluR theory of Fragile X, an autism-like disorder based on a disease that results from a repeat expansion that causes the silencing of the FMRI gene. He points out that in the normal situation, the normal protein created by the FMRl gene, the fmrP protein, prevents the overexpression of protein creation generated by mGluR stimulation. In Fragile X syndrome, the FMRI gene is turned off and the protein is not made, which is a regulatory action on mRNA. This essentially allows the creation of proteins via mGluR to go unregulated and the excess of proteins causes Fragile X syndrome, according to compelling modeling of the disease in mice. Bear proposed that one thing that could be done to correct this is to develop an antagonist to reduce the activity of the mGluR, which reduces the input to the mRNA to create proteins and thereby reduces the proteins. He found that by putting the human gene into mice and then using an mGluR antagonist, he could completely reverse an animal model that had all the characteristics of Fragile X syndrome. This study is in clinical trials and we hope to have the answer by the end of 2011 .

There is similar work to try to understand the anatomy of anxiety. Through animal studies we've learned a great deal about how anxiety develops and how it can be controlled. This has actually led not to a medication treatment but to a behavioral intervention. A key process for controlling anxiety disorders is being able to extinguish fear that arises to a conditioned stimulus. That requires the action of a structure of the median frontal lobes. Through behavioral interventions, one can increase the ability to extinguish anxiety. Alternatively, one can interfere with the memory of a fear stimulus by reexperiencing the conditions that led to the fear and then using medications or behavioral interventions to reduce the emotional reaction to that fear. This turns out to be a very powerful way-by using our understanding of behavior and its effect on biology - to reduce disorders that come about through conditioning.

Figure 3 is a diagram of the structure proposed by Joseph LeDoux for learning about fear and fear conditioning. Interfering with the interaction between the medial prefrontal cortex and the central nucleus of the amygdala makes it possible to control whether or not fear forms.

I'd like to mention briefly how primates are useful in research. The Institute does a significant amount of research on nonhuman primates. Their genetic homology with humans is very important. They have a very large brain, unsurpassed for studies of brain function. Their large behavioral repertoire is more akin to that of humans. Their medication responses are similar to those of humans. The long period of infancy and dependence allows the study of developmental disorders. And at our Institute $70 \%$ of our funding goes toward studies of humans-who are primates as well and are

Figure 3 Medial prefrontal cortex (mPFC) can modulate fear expression via projections to the amygdala. B, basal; $\mathrm{Ce}$, central; IL, infralimbic; LA, lateral

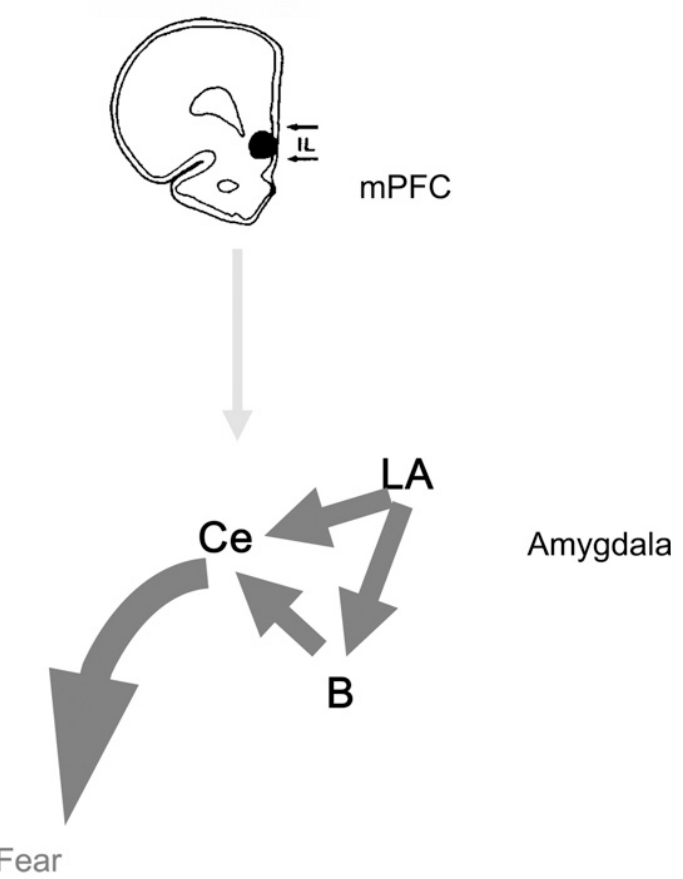


the most studied animals at NIMH. I'll remind you that our mission is to transform the understanding and treatment of mental illnesses through basic and clinical research, paving the way for prevention, recovery, and cure.

I also strongly feel that there are many ethical goods that come out of the use of animals in research. When we understand the biology of systems in any organism we understand general principles that apply to humans because all life has a common origin. This knowledge helps us understand devastating physical and behavioral disorders in humans because we have more insight into the underlying biology of the disorder. And these insights lead to treatments, cures, and prevention that can be applied to humans and other animals. We are developing deep insights into the nature of human beings and I think this is incredibly important. And I believe that the more dominant human beings become on this planet we will desperately need this information if we are to keep the balance of life here.

Finally, a word on animal welfare. I think the NIMH, partially because it really understands the nature of suffering and how it can develop, has always paid a lot of attention to animal welfare. I believe we and this community pay more attention than almost any other segment of human-animal interaction (the only exception to that, I think, is what goes on in veterinarians' offices). But if you think about almost every other use or interaction of humans with animals, animals come out worse, in many cases much worse. To pay attention to animal welfare through the Animal Welfare Act has been an incredible benefit both to animals used in research and ultimately I think to us, by helping us to understand the illnesses that this Institute studies.

\section{References}

Bear MF, Dölen G. 2008. Role for metabotropic glutamate receptor 5 (mGluR5) in the pathogenesis of Fragile X syndrome. J Physiol 586: 1503-1508.

Bredy TW, Zhang TY, Grant RJ, Diorio J, Meaney MJ. 2004. Peripubertal environmental enrichment reverses the effects of maternal care on hippocampal development and glutamate receptor subunit expression. Eur J Neurosci 20:1355-1362.

Eriksson PS, Nilsson M, Perfilieva E, Johansson U, Orwar O. 1999. Enriched environment increases neurogenesis in the adult rat dentate gyrus and improves spatial memory. J Neurobiol 39:569-578.

Gould E, Tanapat P. 1999. Stress and hippocampal neurogenesis. Biol Psychiatry 46:1472-1479.

Meaney MJ, Szyf M. 2005. Maternal care as a model for experiencedependent chromatin plasticity? Trends Neurosci 28:456-463.

Meyer-Lindenberg A, Weinberger DR. 2006. Intermediate phenotypes and genetic mechanisms of psychiatric disorders. Nat Rev Neurosci 7:818-827.

Sapolsky RM. 2004. Is impaired neurogenesis relevant to the affective symptoms of depression? Biol Psychiatry 56:137-139.

Weaver IC, Cervoni N, Champagne FA, D’Alessio AC, Sharma S, Seckl JR, Dymov S, Szyf M, Meaney MJ. 2004. Epigenetic programming by maternal behavior. Nat Neurosci 7:847-854.

van Praag H, Kempermann G, Gage FH. 1999. Running increases cell proliferation and neurogenesis in the adult mouse dentate gyrus. Nat Neurosci 2:266-270.

\section{Animal Models in Immunology and Transplant Medicine}

\section{Linda Cendales}

am going to talk about how the Emory Transplant Center (ETC) established a comprehensive program for transplantation and how the use of animal research for our advances is crucial. I'm also going to share with you a little bit of my academic interest, vascularized composite allotransplantation, which basically is the allotransplantation of any vascularized peripheral tissue (such as skin, bone, nerve) as a functional unit for tissues that cannot be reconstructed in any other way. An example of it is a hand.

The risk-benefit ratio in transplantation is the risk that the patients take when they choose a life-saving or qualityof-life transplant versus the risks of taking immunosuppression with all the [associated] complications (including infection, malignancy, and death).

I'll share with you our approach to transplantation at the ETC. Figure 1 shows what is often called the Larsen Circle of Life, with discovery at the lab, our translational research in nonhuman primates at the Yerkes National Primate Research Center, and our clinical applications. Examples of the projects that we have in the lab are antigen presentation, memory, and protective immunity. Examples of our translational studies in nonhuman primates include protocols on memory, xenotransplantation, and novel immunosuppression. Some of our novel clinical trials include studies in hand, islet, and kidney transplants, and pediatric transplantation.

Briefly, the alloimmune response has [several] signals. Signal 1 is where cyclosporine, for example, works. Signal 2 is where I am going to focus more in this talk.

When we transplant an organ, we bring with it antigenpresenting cells (APCs), which activate the recipient's T cells. Studies have shown that blockage of this stimulation reduces rejection. Studies in mice inducing diabetes and transplanting islets in the renal capsule [showed that] block- 


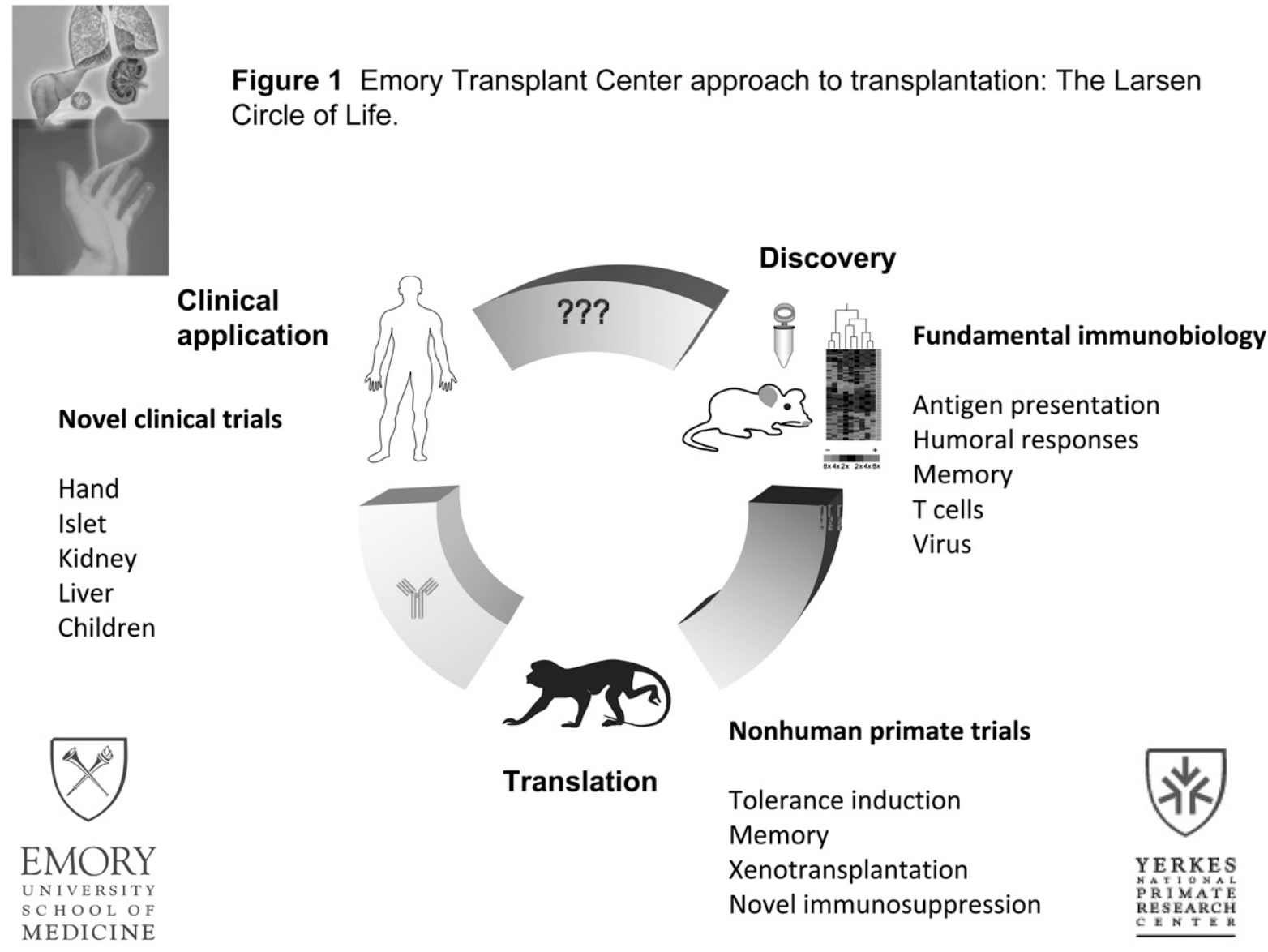

ing the B7 family with CTLA4-IG reduces rejection and produces long-term survival of islets in diabetic mice. The investigators did a control and the mice rejected (Lenschow et al. 1992). Then Parker and colleagues (1995) showed that it was possible to prolong rejection-free diabetic mice after islet transplantation by using anti-CD154 (Parker et al. 1995). The next step was by Larsen (Larsen et al. 1996): he blocked both signals CTLA4-Ig and CD154 in the APC and the $\mathrm{T}$ cell and showed longer rejection-free survival. In subsequent studies by Allan Kirk (Kirk et al. 1997) in a nonhuman primate model of kidney transplantation, all the controls without immunosuppression rejected within the first week. When he gave CTL4-IG alone, the monkeys were rejectionfree for longer, but did reject within the first 30 days. Administration of anti-CD40, which is [another] component of the costimulation of the blockade alone, further deferred rejection in the monkeys. They had rejection by 3 months, but reversed it and prolonged the kidney transplants free of rejection.

The next step was to give both together. Again there was prolongation of kidney transplant with costimulation blockade. Next was the rationale of translating - making a medication that would include this concept that has been studied in animals for 10 years but making it more potent. In this study, Larsen and his group (2005) at the ETC developed a more potent medication called Belatacept, which is based on costimulation to block the CTLA4-Ig. Through animal experimentation they were able to identify the amino acids necessary to change to improve efficacy. This medication has been translated to the clinic and is currently in phase III studies in clinical trials (Vincenti et al. 2005). Among the benefits of this medication, it's more specific to the immune response so instead of, for example, cyclosporine, which depletes the immune system in a more general way, costimulation blockade and Belatacept block only a signal in the alloimmune response, making it more specific. Another benefit is that it's given once monthly versus a number of medications that transplant patients need to take daily.

Based on these advances in immunosuppression as well as in transplantation and microsurgery, we have been able to move forward to include patients for both quality-of-life and life-saving transplants. This is where vascularized composite allotransplantation has emerged as a partner in the field of transplantation. But like any emerging field, we have faced challenges. One of the challenges was, initially, the absence of clinically relevant animal models in which we could not only understand the specificities of this transplant but also apply novel immunosuppression before its translation to the clinic.

We wanted to establish a nonhuman primate model because, in the era of biologic immunosuppression, the most relevant clinical model is in nonhuman primates to evaluate novel approaches. Taking into consideration, as we all do, the well-being of our animals and their social development, 
we designed a transplant that includes every single tissue present in any composite allograft_bone, nerve, tendon, vessels, and skin. The transplant involves taking a piece of a forearm from one animal to another.

One of the benefits is that, even in the case of graft loss, the animals do not lose any function. In fact, as soon as they recover from the anesthesia, they go back to the cage and have full use of the upper extremity. Another benefit is that we can biopsy the tissues serially without graft loss and the animal does not experience any dysfunction either in the upper extremity or in the hand.

We started with our first group, which was without immunosuppression. As in humans, all the monkeys rejected within the first week. Then we applied immunosuppression similar to that for kidney transplantation. When we started decreasing the immunosuppression to evaluate rejection of these transplants, what we saw was comparable to what we were seeing in human hand transplants: with a decrease of immunosuppression, a rash in the skin develops. This is a benefit compared to other organ transplants as we can visualize the transplant. The rejection is well demarcated or circumscribed to the transplant. Similar to humans, the transplants in the nonhuman primates show comparable hair growth and similar magnitude and distribution of the $\mathrm{T}$ cell infiltrate in rejection.

Something novel that we have established is a nonhuman primate card to study the gene expression of markers that have been increased at the time of rejection in other transplants. We are comparing it with the histology to understand better the mechanisms of rejection.

In summary, so far, in terms of vascularized composite allografts we have been able to establish a nonhuman primate model for the study of this particular transplant, which is responsive to immunosuppression. We have moved forward with novel immunosuppression applications for clinical translation.

But we continue with our challenges and certainly the burden of immunosuppression is an important one. We have moved forward with novel approaches and are evaluating costimulation blockade based on Belatacept, the medication developed at Emory. Briefly, monkeys that received a transplant without immunosuppression rejected within the first week as we saw in the prior slide. An animal treated with Belatacept is rejection free at this time.

Our first clinical application for vascularized composite allograph is hand transplantation for the reconstruction of below-the-elbow amputations. We are actively recruiting patients. Our program is in collaboration with the Atlanta Veterans Affairs (VA), which is the only VA hand transplant program in the United States. Our VCA Emory-VA Program is based on a multidisciplinary approach: we have the Emory Transplant Center, plastic surgery, neuroscience, a crucial component of our program is the Division of Animal Research and the Yerkes National Primate Research Center, and all the veterinary services. We have immunology, pathology, mental health, hospital services, infectious diseases, pharmacy, prosthetics, the Georgia Tech School of Applied Phys- iology, Life Link (our organ procurement organization), public health, VA Research and Development, oral and maxillofacial surgery, radiology, public relations, the Mason House (where we host our transplant patients), the Simulation Lab, Pastoral Care and Bioethics, as well as the Atlanta Clinical and Translational Science Institute-all working together to move the field forward in a systematic way and offer our patients outcomes that are the same as or better than those we have seen so far.

I want to share a couple of patient stories to show the clinical translation of the research that we do from the bench to the bedside and the importance of its application. First is the story of a patient who has been approved to move forward with a bilateral hand transplant because she wants to take care of her kids and she would like to go to the bathroom independently. Our next patient approved for our program is a veteran who lost the lower extremity and the right upper extremity in the Iraq conflict. I will share with you our current outcomes.

I was involved in the first two hand transplants in the United States and I'll describe the second patient that we did in the United States. His transplanted hand was matched for skin pigmentation, gender, blood type, and size. He has discoloration in his transplanted hand, which is similar to what we saw in our nonhuman primates-very well demarcated and circumscribed to the allograft. A 1-minute video showing the outcome was taken when I was still taking care of him in Louisville. Every single activity shown in the video he could not do with his body-powered prosthesis. He is a construction worker and was able to return to full work by month four.

All these patients have been shown to acquire or regain sensation. It's not normal [sensation], but it allows them to feel surfaces and differentiate between rough and smooth surfaces and between hot and cold, so it's a functional protective sensation. They recover sensation to the fingertips within the first 6 months, which is more rapid than we see in replantation.

Some of the advances so far: We started with the lack of an animal model in which to carry out our experiments to be able to translate our findings to the clinic. We established a nonhuman primate model that is responsive to immunosuppression. We started with a burden of immunosuppression. We have been able to minimize regimens. We have also established a comprehensive program from the bench to the bedside in our field of transplantation.

Reviewing the Larsen Circle of Life, we incorporated this new partner in transplantation, vascularized composite allotransplantation, into our ETC approach to transplantation, including hand and other potential clinical applications for the reconstruction of tissues that cannot be reconstructed with autologous tissue. We integrate our discoveries in the lab, our observations through our translational research in nonhuman primates, and our clinical applications for the improvement of human health. The questions generated in the clinic cycle back to the lab and our animal models to complete the circle of life. 


\section{References}

Kirk AD, Harlan DM, Armstrong NN, Davis TA, Dong Y, Gray GS, Hong X, Thomas D, Fechner JH Jr, Knechtle SJ. 1997. CTLA4-Ig and antiCD40 ligand prevent renal allograft rejection in primates. Proc Natl Acad Sci U S A 94:8789-8794.

Larsen CP, Elwood ET, Alexander DZ, Ritchie SC, Hendrix R, Tucker-Burden C, Cho HR, Aruffo A, Hollenbaugh D, Linsley PS, Winn KJ, Pearson TC. 1996. Pillars article: Long-term acceptance of skin and cardiac allografts after blocking CD40 and CD28 pathways. Nature 381:434-438.

Larsen CP, Pearson TC, Adams AB, Tso P, Shirasugi N, Strobert E, Anderson D, Cowan S, Price K, Naemura J, Emswiler J, Greene J, Turk LA, Bajorath J, Townsend R, Hagerty D, Linsley PS, Peach RJ. 2005. Rational development of LEA29Y (belatacept), a high-affinity variant of
CTLA4-Ig with potent immunosuppressive properties. Am J Transplant 5:443-453.

Lenschow DJ, Zeng Y, Thistlethwaite JR, Montag A, Brady W, Gibson MG, Linsley PS, Bluestone JA. 1992. Long-term survival of xenogeneic pancreatic islet grafts induced by CTLA4lg. Science 257:789792.

Parker DC, Greiner DL, Phillips NE, Appel MC, Steele AW, Durie FH, Noelle RJ, Mordes JP, Rossini AA. 1995. Survival of mouse pancreatic islet allografts in recipients treated with allogeneic small lymphocytes and antibody to CD40 ligand. Proc Natl Acad Sci U S A 92:9560-9564.

Vincenti F, Larsen C, Durrbach A, Wekerle T, Nashan B, Blancho G, Lang P, Grinyo J, Halloran PF, Solez K, Hagerty D, Levy E, Zhou W, Natarajan K, Charpentier B; Belatacept Study Group. 2005. Costimulation blockade with belatacept in renal transplantation. N Engl J Med 25:770-781.

\title{
Animal Models Facilitate Rapid Responses to Emerging Infectious Diseases
}

\author{
Michael Kurilla
}

was asked to speak about infectious diseases and animal models in the context of how we can, now and in the future, deal with emerging infectious diseases. I think it's important to point out that whereas we share a lot of our pathophysiology with animals in terms of diseases that humans get, we also share infectious diseases with animals. I think this makes infectious diseases unique in terms of both what we do for humans and applications for animals, in terms of economic impacts, agricultural use of animals, and companion animals.

First, a bit of historical context. We have a tendency in this country to make premature declarations of victory over a lot of things, and over 40 years ago we thought we had licked infectious diseases. There was quite a bit of hubris at the success we had with treatments being developed in terms of antibiotics. And there was a lot of hope that infectious disease practice in medicine was going to devolve to an annual update of a bug-drug book. There was also active discussion in the medical community as to whether the specialty of infectious diseases would be gradually phased out and discarded over time. But the reality, unfortunately, was that [that thinking] was very much premature.

An article in Nature (Jones et al. 2008) identified at least 335 infectious diseases that emerged between 1940 and 2004. What's interesting to look at from that article are the global trends, which you can see in the four charts of Figure 1 that depict globally where this is occurring. Panels A and B list zoonotic diseases, from wildlife and nonwildlife, respectively. It is important to keep in mind that infectious diseases are travelling back and forth between humans and animals and that animal reservoirs represent a major source of infectious disease [for humans].
At the National Institute of Allergy and Infectious Diseases (NIAID), we have a dual mandate, which is slightly different from most other $[\mathrm{NIH}]$ institutes. We have the typical mandate of other institutes, which is to maintain and grow a basic and applied research portfolio in [our] mission, primarily microbiology and immunology. But importantly, we are the one institute that can, without doing anything specific, find ourselves facing brand new diseases that we must rapidly respond to and try to bring to bear as many resources as possible to bring these new diseases under control.

[I'll offer] some highlights. In 2001, anthrax was less than a bang and more a whimper, but this belies the fact that pieces of paper and the postal system turned out to be a tremendous delivery device to cause not only in some cases death but also widespread panic and fear. In 2003, 2 years later, SARS (sudden acute respiratory syndrome) gave us an example of a disease that was wholly unexpected to arise. There was very little in the research base that anticipated something with the attributes of SARS. [The experience reflects] the ill-defined and unanticipated interplay between culinary preferences and animal handling in certain parts of the world that allowed the disease to emerge. We dodged a bullet on SARS, I would have to say. The mortality rate for the disease is $10 \%$, which doesn't sound terribly frightening except that, to put that in context, the 1918 influenza pandemic, which was responsible for 50 million deaths around the globe, had a fatality rate of only $3 \%$, so $10 \%$ mortality is quite high. We were lucky with SARS because it turns out to be a disease that is not contagious until after you've become symptomatic. So if you can isolate everyone who's been exposed and let the disease run its course, you can stop transmission in its tracks. With flu, on the other hand, you're 


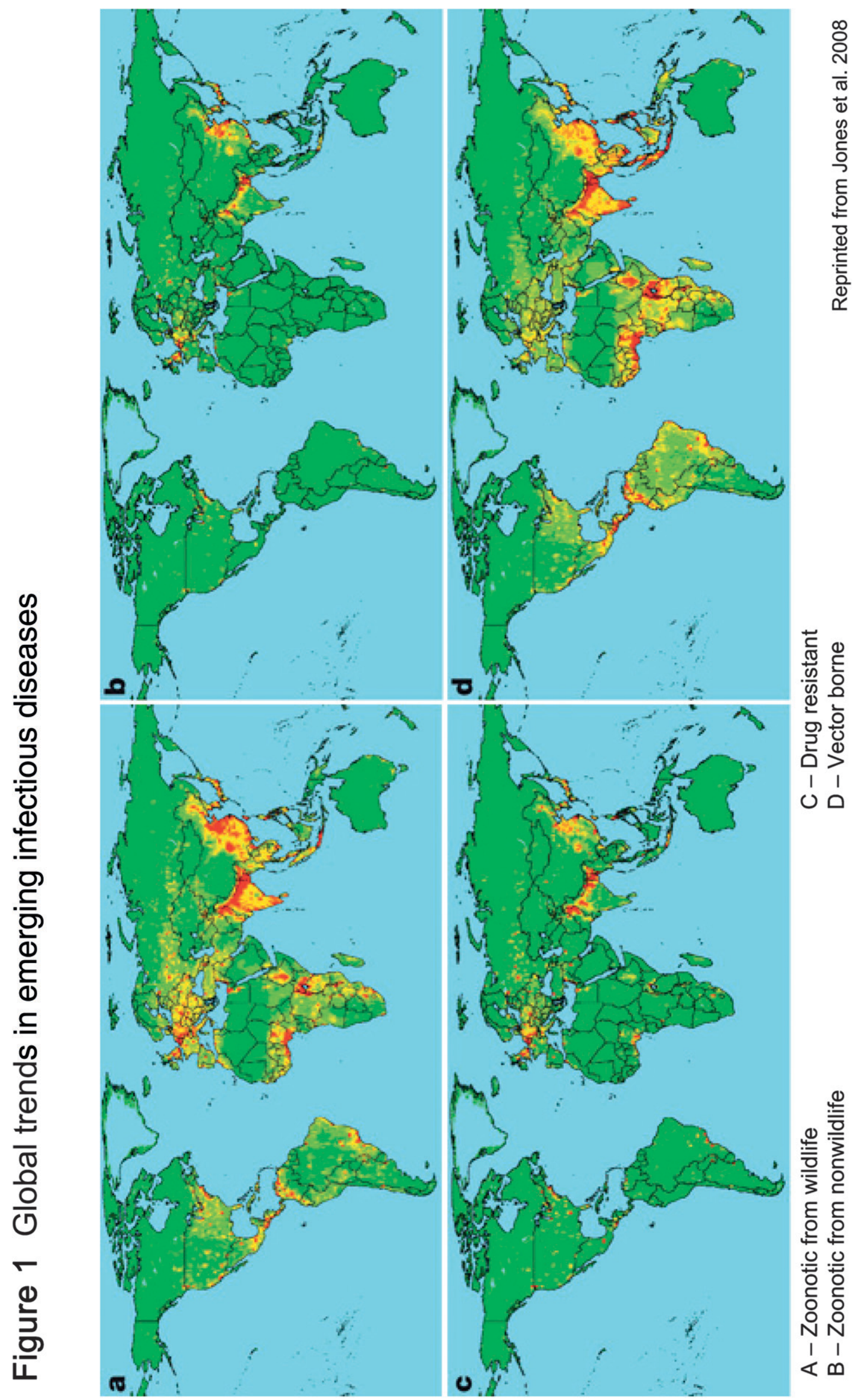


contagious long before you even know you have an infection, which is why it's so insidious in terms of trying to stop its spread.

Earlier this year, swine flu became the latest to emerge in a very unexpected way. As of March 2009 we knew there was an outbreak in Mexico. The recognition of H1N1 in the United States was actually a happenstance of ongoing preparations for what was and still is an anticipated H5N1 (avian flu) pandemic. An H1N1 diagnostic test being evaluated in a San Diego hospital for the CDC turned out to be an apparent outlier-it identified the influenza as type A but would not subtype a specific HA type, which was what the test was supposed to do, to distinguish the seasonal $\mathrm{H} 1 \mathrm{~s}, 2 \mathrm{~s}$, and $3 \mathrm{~s}$ from the more problematic and concerning H5s. This isolate didn't subtype at all, so it was sent back to the CDC as an anomaly, something that needed to be looked at in case it represented a void or a hole in the diagnostic test. It happened that the CDC was at the same time evaluating the Mexico cases and recognized that the San Diego case of the atypical flu was the same as the Mexico cases that they were beginning to evaluate and that's how we knew H1N1 was in this country. If we had not had that diagnostic test undergoing evaluation, we probably would have taken at least another month before there were widespread outbreaks that would have raised suspicion. But this was the situation in March of 2009. By November, it was pretty much everywhere on the planet. These are only laboratory-confirmed cases, [of which] over 600,000 have been identified. The estimates for the United States are probably somewhere around 60 million people actually infected with H1N1.

At the same time, in the pharmaceutical arena there is a pipeline issue: we're seeing a declining number of drugs being brought to the market. There are a complex array of reasons for this but the simple reality is that relative to other therapeutic areas, infectious disease applications simply do not muster sufficient commercial viability to allow companies to allocate resources in that area. It should also be mentioned that the randomness of the way infectious diseases can emerge and disappear periodically does not lend them well to development programs that are really looking for chronic dosing for chronic diseases.

At NIAID, we have applied a lot of NIH inputs, basic research, and the whole area of genomics, which is just a term for the overall -omics revolution in general. We've also done a lot of expansion of clinical research capacity. All of this is directed toward the development of new, safer, and more efficacious vaccines, therapeutics, and diagnostics. Long-term research, where we're looking for the field to really head in, is on the ability to create broad-spectrum therapeutic platforms. We've tended to do "one bug, one drug" in the past and we feel that that's not economically viable. It's also not very efficient because of the rate at which these diseases appear. Multivalent vaccine platforms allow for more rapid production. We've also seen, with emerging science, immune-enhancement strategies that provide adjuvants for vaccines and may also serve as stand-alone immune modulators that can either enhance immune responses to infectious diseases or decrease adverse immunologic reactions that underlie a lot of severe disease manifestations. And finally, while it's been very successful in a wide array of other therapeutic areas, the application of monoclonal antibodies has been less applied in the infectious diseases. With only one licensed infectious disease-specific monoclonal, we see potential to really move the field forward.

In terms of product development, where we're looking for new clinical indications, new therapeutics, and new vaccines, there are two major elements that we have to combine. We have to have clinical research that can define the pharmacokinetics of drugs or, if we're talking about vaccines, understand the immunogenicity. And we need a considerable amount of animal model development to move the development forward. We work with the FDA, particularly when we're talking about biodefense applications, because there's very, very limited expertise in a lot of these areas. What's needed from the animal model side is to help us define the pathogenesis and natural history, because many of these diseases are new or infrequent, they're very poorly understood, and we may not have adequate human clinical data with which to make a lot of progress toward drug development. The animal models help us to define the pathogenesis and the natural history.

As you'll see later, in order to get licensing under what is now referred to as the Animal Rule, we have to demonstrate that the animal disease we're seeing is comparable to the human disease. This is something that should not be looked upon lightly. Many infectious diseases cause different patterns in animals than in humans, so it's very important to be able to identify that the disease we're trying to treat in the animal matches what we see in the human. Then we develop animal efficacy studies, which can be used in terms of the Animal Rule for pivotal animal efficacy studies that will support licensure of the drug for the human disease.

So our animal model development program has a wide array of activities ongoing, [including] one particularly for small animals such as mice, [which] we use for in vivo screening feasibility studies to evaluate a lot of candidate therapeutics or vaccines. As I mentioned, we characterize the natural history of the disease and then we develop efficacy models....

What we've seen over time is an evolution in the animal models that we use. Typically, historically, a lot of animal models in infectious diseases have simply been infection modelsyou infect the animal with the organism, and it may or may not cause disease. We frequently see this-there are a lot of infections that are subclinical or asymptomatic in humans as well. So we've moved away from simple infection models to true disease models, in which the organism causes disease in the animal that looks similar to the disease that occurs in humans.

For evaluation of therapeutics, historically we used animals to evaluate early-stage bioavailability and metabolism issues. Again, we're using these more to define how the drugs work and their applicability in terms of humans. In the animals now for infectious diseases, PK/PD (pharmacokinetics 
and pharmacodynamics) drive the clinical parameters. PK/ PD derived from animal models for the infectious disease therapeutics go a long way toward defining the dose and dosing schemes that will be used in humans. [But there are] two caveats. What we've seen in this process is that there are severe limitations to mirroring models for human relevancemost of the time, the infectious disease that occurs in mice is not the same as the infection that occurs in humans, even with the same organisms. This leads to the second caveat, which is unfortunate: that for many nonmurine systems there is a limitation in the reagents available to conduct the type of detailed studies needed.

In general, we have two classes of countermeasures that we try to focus on. For one, we have a known licensed product-it's approved for a specific indication, but it's unapproved for a desired indication. You see this quite frequently with antibiotics - they're out on the market, but they're not approved for specific diseases because there's [not] enough data. They may be efficacious or the efficacy is unknown or questionable. The presumption is that you may have to go to a dose that has significant adverse, toxic effects that would preclude use. So those [countermeasures] are for the known licensed products and we develop those that go through a pathway known as a surrogate marker rule that I'll talk about shortly. The second class is the novel unique medical products. These are designed specifically for the CBRN (chemical, biological, radiological, and nuclear) applications for which we would like to have drugs that we know work against these agents. We lack the ability to do a traditional phase III efficacy trial because there are insufficient numbers of human patients with these diseases and we hope that there will never be sufficient number of patients with these diseases to undertake this type of trial.

I'll give you one example of an animal model that we have developed, a rabbit model to evaluate the anthrax vaccine. We know the vaccine works in a preexposure prophylactic manner, that's what its licensed indication is. However, there have been data for a long time that anthrax actually has postexposure prophylaxis capability - that is, after you are exposed to anthrax spores, there's a period of several days when you can be vaccinated and the vaccine will manifest a benefit over not being vaccinated at all.

Anthrax is not the only vaccine with this property. Most people are probably familiar with the rabies vaccine-you typically take it after you've been exposed to rabies and it displays postexposure prophylaxis. Smallpox is another with demonstrated postexposure prophylaxis. In Figure 2, the control line represents the rabbits exposed to anthrax spore with no treatment. It's very rapid, a little faster than in humans. Since people who would be exposed to anthrax are going to be given antibiotics, you need to show that there's a benefit [of the vaccine] over the antibiotics alone. In this case, the rabbits are treated for 7 days with antibiotics only; about $50 \%$ of them survive, $50 \%$ succumb. But with the vaccine you get nearly $100 \%$ protection, demonstrating postexposure prophylaxis efficacy. These data will be used in support of licensure for a postexposure prophylaxis indication for the anthrax vaccine that is currently in the strategic national stockpile.

Figure 3 shows the development of a nonhuman primate aerosolized pneumonic plague model that we have developed for testing antibiotics. Again, the vertical line shows that the

\section{Figure 2 Anthrax vaccine postexposure prophylaxis efficacy. Abx, antibiotics}

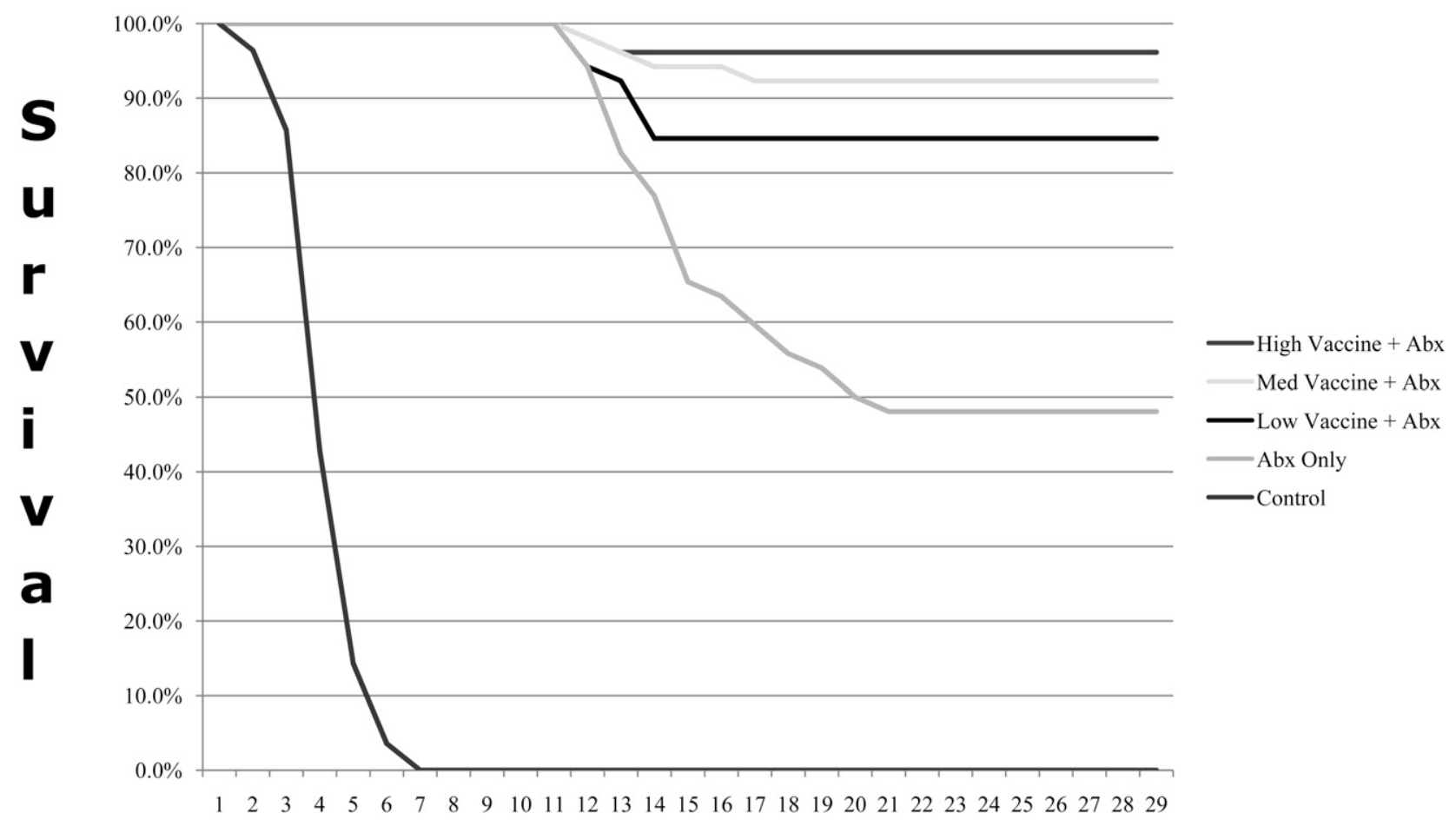




\section{Figure 3 Plague antibiotic efficacy studies}

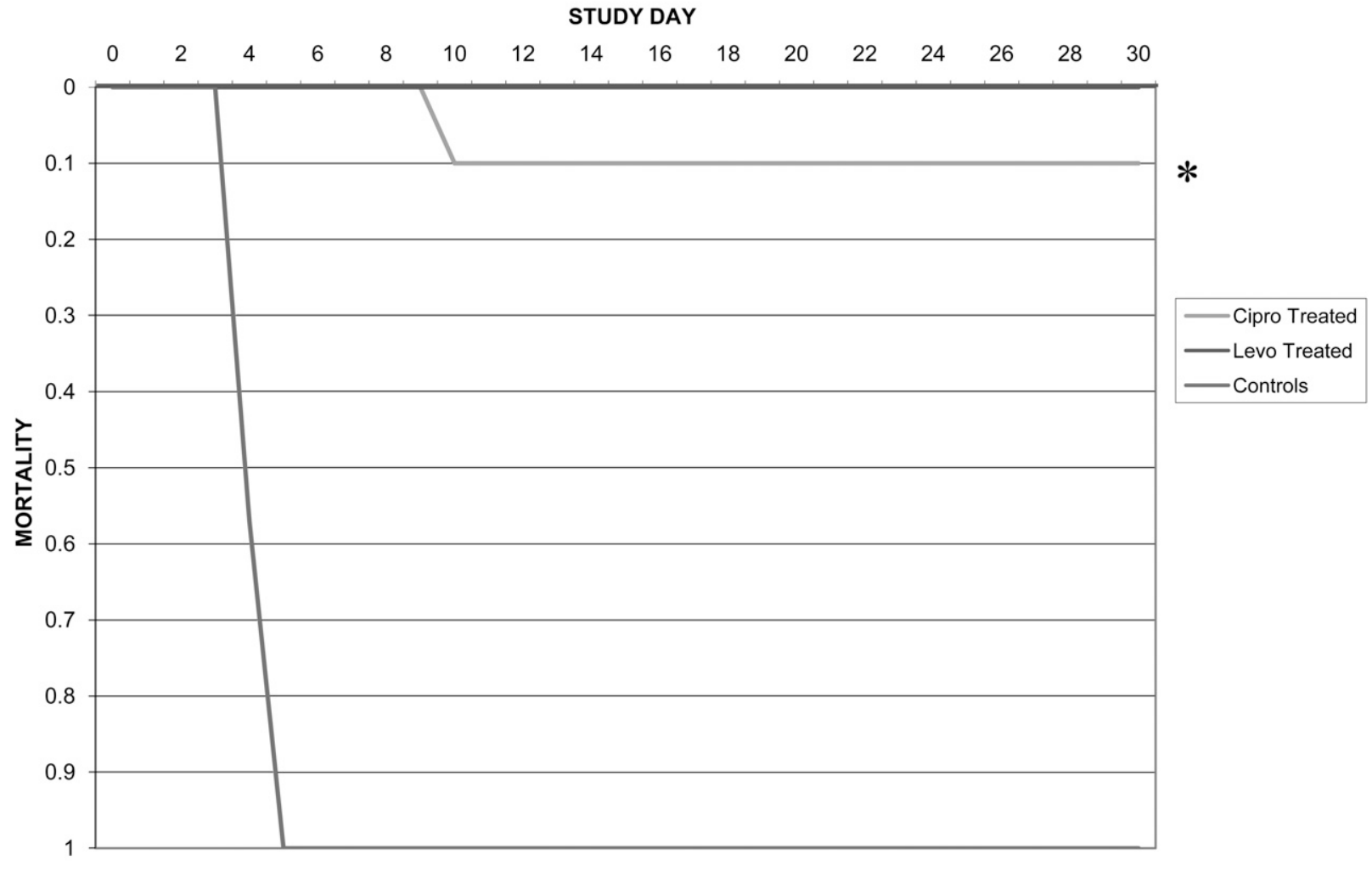

*Death due to catheter failure, unrelated to Yersinia pestis infection

control animals exposed to plague succumb in a very rapid fashion. This is probably not too dissimilar from what is seen in humans-usually with aerosol plague, in about 4 days you are dead. What you can see here is that, using either ciprofloxacin or levofloxacin, there is nearly $100 \%$ survival (the one animal that succumbed was because of a catheter failure that precluded getting the drug into it). These data are being submitted to the FDA in support of licensure of an additional clinical indication for these drugs to support their use against plague infections. Conducting plague studies in humans has been attempted, but it's very difficult. There's one area in the world where there might have been sufficient cases of plague: Madagascar. Unfortunately, there's a fair amount of overlapping other diseases [there] that precludes being able to conduct a plague study, although it has been attempted. Traditional phase III trials are just not feasible in humans.

The use of animals to obtain these indications involves some pretty complex regulatory approaches.... We follow two of these in the FDA, the surrogate marker rule and the Animal Rule. The surrogate marker rule was put forth in the early 1990s; it [calls for] approval based on a surrogate endpoint or an effect on a clinical endpoint other than survival or irreversible morbidity. This is most frequently done in terms of HIV, for example, using CD4 cell counts. For cholesterol-lowering drugs, you would use cholesterol level as the endpoint rather than the actual disease outcome of elevated cholesterol. This [approach] has been used to obtain approval for antibiotics, so it is largely used for drugs that are already approved and you're seeking additional indications. The idea is that the animal PK/ PD data serve as a surrogate for the PK that can bridge to the PK data from human studies. There is a very wide safety margin with these drugs-you know the exposure levels and the PK you obtain in the humans, so you show what is needed to effect a successful treatment in the animal [and] that you would be able to obtain that [outcome] in humans, and that's the basis on which the approval is granted.

The much more difficult one is the Animal Rule, which is newer and still being test-run to move forward. The idea behind the Animal Rule is that human efficacy evaluation is either not technically possible or not ethically feasible. There's no way we could ever do an experiment that would meet the standard of what we typically think of for a classical phase III safety and efficacy study. It requires acceptable animal efficacy data and this is extremely rigorous. You still need human clinical data. If you're looking at a vaccine you require human immunogenicity data, and if you're doing a drug you require human PK data. In the case of vaccines you demonstrate that the vaccine is efficacious in the animal and you demonstrate that the correlate of protection, the immunologic response of the animal, is manifesting that it is responsible for that protection from the vaccine, and then demonstrate that same response to an equivalent or higher level in humans. That's the basis for the bridging. In terms of PK, again you are using the PK data to show that if you 
obtain the equivalent PK exposure levels in humans you can reliably predict that you'll have successful treatment. You're still obligated under the Animal Rule to demonstrate safety, so there's still human testing of the drug or vaccine in larger numbers than what people had anticipated. But you're doing those purely for safety, not for efficacy.

Finally, the approval comes with lots of restrictions in terms of use and postmarketing studies that require extensive follow-up. The only two instances where the Animal Rule has been invoked have been for chemical applicationspyridostigmine bromide against a nerve agent and the Cyanokit have both been approved under the Animal Rule. As of yet, no biologicals have been approved. The Animal Rule sets a very high bar-you must understand not only the disease caused in the animal and in the human but the morbidity and/ or mortality of that disease. The Animal Rule specifically states that the effect of the intervention must demonstrate [efficacy] with sufficiently characterized animal models for more than one species-some people talk about the "twoanimal rule." The animal study endpoint must be related to the desired benefit in humans. The PK or immunogenicity data from the animals that show demonstrated efficacy must allow selection of an effective human dose so that you can create your safety database at that specific dosing level. Without the Animal Rule, novel treatments for anthrax or Ebola, for example, would be essentially impossible.

In terms of infectious diseases, I think we've finally reached a grudging acceptance that we're probably not going to ever defeat them. They have an extraordinary capability to basically apply Darwinian principle far faster than we can ever hope to. So regardless of what we do, regardless of the interventions, they'll just keep coming back at us and it will be a perpetual struggle.

If anyone is interested further in information about our activities, we have a webpage devoted to information resources that I encourage you to visit (www.niaid.nih.gov).

\section{Reference}

Jones KE, Patel NG, Levy MA, Storeygard A, Balk D, Gittleman JL, Daszak P. 2008. Global trends in emerging infectious diseases. Nature 451:990-993.

\section{Animal Models and Basic Science—Bench to Bedside: Discussion}

Questioner: Michael, I am assuming that all the research you've described must take place in a biosafety level (BSL)-4 or -3 facility. The research institutions in the Northwest are having ongoing struggles with public understanding of BSL-3 and -4 facilities and the importance of allowing them both to be built and to continue their research. What advice do you have?

Dr. Kurilla: I would first say that the Pacific Northwest is not unique in that regard. We've seen a lot of confusion and misinformation. This is one area where I think public information is critically important. There is somehow the impression that if we weren't doing this type of research, there wouldn't be a problem and yet I struggle when people see something like SARS, which caught most scientists completely off guard in terms of the potential. We knew coronaviruses existed, we knew they were a minor cause of upper respiratory infection-and yet something so severe that could come out of nowhere was not on anybody's radar screen. The difficulty here, I think, is that we live in an extremely short time frame. I've had discussions with people about what they mean when they say medium or long term; for many, "long term" is next year. So the importance of establishing a fundamental, sustainable, robust research base that is asking important questions that are not going to be answered tomorrow but will be very valuable for the future as things arise- that can't be overstated.

When SARS arose, I think we had at most two investigators that were funded to study coronaviruses, but having those two investigators was extremely valuable because they became immediate subject matter experts and were very instrumental in making correct decisions about what needed to be done. Unfortunately, in economic times such as we now have, focusing on long-term preparedness efforts is a tough sell overall and people would prefer just not to deal with it-and then blame somebody when something bad happens. People ask me why I continue to fund programs directed toward SARS animal model developments, vaccines, diagnostics, and therapeutics. The simple reason is that if SARS ever reemerges, a lot of people are going to ask me what I've been doing all this time. In the absence of that it is very, very difficult.

We have, with some of our development programs, focused on community engagement. I will say that typically scientists are in general not very facile or familiar with the types of things that community engagement requires, which is very different from engaging with the scientific community. Most of the time, in my experience, scientists tend to present the same sort of message to every group of people they talk to, whether they're talking to other scientists, politicians, 
aid agencies that are interested in what they are doing, or concerned community groups. The message has to be tailored because people are looking for different things, they have different questions, and you can't diminish or disparage the questions. There's no such thing, I think, as a naïve question; it's simply that there's a lack of adequate information because there's too much competition for the small amount of information space that we have available to take in information. The only advice I can give you is to engage... professional communicators, people who understand this a little better. You almost need to take it away from scientists who may feel attacked and get into a defensive mode.... We have found that our community liaison people are much better at being able to identify and articulate those concerns and issues and to present the same message that scientists give but in a slightly different way.

Questioner: This question is for Richard Nakamura. You pointed out tangible evidence of changes that occur as a result of enrichment strategies and I think that raises some very important questions that we need to address seriously and scientifically. Given what you described as the two-way street-the epigenetics, the potential for research-complicating variableshow do you control for the changes that might occur with enrichment? This community is really committed to pursuing the enrichment concept and strategies, but how do you merge that with the scientific concerns - the project designs and controls necessary to avoid unintended outcomes?

Dr. Nakamura: You have to control for everything, and one way you can look at this is not necessarily that the environment is enriched for some mice or rats so much as unusually impoverished in the average laboratory environment. I think we all have to better understand what causes stress or trauma to laboratory animals. I don't think there has been sufficient emphasis on that aspect of animal care. But as a result of some of our research we're beginning to understand that better and how to lower stress for laboratory animals. I think in an important way, what we haven't recognized so much is that the amount of noise that you have in an environment is very important for certain animals and they can be stressed if there is an unusual amount of cleaning activity going on. So sometimes what we believe is healthful—sanitation, cleaning, cage changing, and so forth - if done at too high a rate can actually produce stress and hurt the animals more than a lower level of animal care and cleaning that keeps noise and light pollution down. Animals are better off then. I think we do need to provide and think about more research in this area.

Also we have to be careful not to anthropomorphize. Very often it's what we see and feel comfortable with in our own environment that we try to impose on animals, which often isn't the best for the animal. I remember a friend who had been doing bird studies and could not get her birds to mate and reproduce until she allowed smells and quietness and cages that kept the odor of the birds and finally convinced the animal care staff that this was, in fact, healthy for the animals. We need to pay attention and look both ways to create appropriate environments for animals. And I think we'll learn for ourselves what's better for human beings as well.
By the way, this has been a major interest at NIH, where we have been doing group housing, providing outdoor housing for primates and others, and thinking about what is a better environment for our murine studies.

Questioner: Michael, you made a comment that monoclonal antibodies are less applied to infectious diseases and I was wondering if there was a general take-home message on that?

Dr. Kurilla: I think it's part of the overall... way the pharmaceutical industry prioritizes what they do. It's based on a complex formula that's referred to as NPV, net present value. You cost up everything that you have to invest in bringing a drug to market and then you subtract that from the expected revenues from being able to sell the drug with appropriate future discounting because a dollar 100 years from now is worth less than a dollar today.... You get some idea of where discounting comes in.

Unfortunately, monoclonals have a fairly large upfront investment cost relative to a lot of other things, just to get them even to begin clinical testing in humans. There's a very complex cell banking procedure that must be undertaken before you can begin any clinical R\&D. You're making bets very, very early on what monoclonal you think is going to work. Then you have to invest a lot of money before you even get to the point of testing to see whether it can get into humans and have any chance of working. In general I think that monoclonals have tended to be less favored in terms of infectious diseases because of their inherent acute dosing and limited applicability for an inflammatory type of application, where somebody's going to be taking an injection every week or every month for the rest of their life-the economics are a little different. If you're talking oncology products, the pricing is much, much different.

Questioner: This is for both Richard and Linda. Richard, you made what I thought was a very profound point in your talk: that the world has changed from what we grew up in, where it was nature versus nurture for behavior. In fact the interaction [is now] what we have to target and understand. The development of the -omics era in genomics and geneticsalthough we supposed that would simplify our understanding - in many ways adds a level of complication in efforts to unpack the interaction between those two. One could see this, probably, very simply in your work, Linda, where the question would be, Are some patients simply better candidates because of their genetic makeup, not so much in terms of their MHC typing but in terms of their mood, in terms of their personality types? Some individuals are simply going to do better at transplant experiences than others, based on the kinds of things you're talking about, Richard.

Dr. Nakamura: I completely agree. We're also talking about personalizing care so that we can make better predictions about which patients will need what interventions. Who will do better with behavioral interventions? Who will do better with medication interventions? We already know that this is the reality and now we are beginning to find out, through genetics and other kinds of understanding, phenotypes relevant to genetics for personalizing; and [we're] getting better at predicting who will respond to what kind of treatments. 
Dr. Cendales: I would add that all of us, as with psychosocial factors, are exposed throughout our lives to different pathogens that make up the immunological history of our immune system. So all of us will likely respond differently to transplantation and to immunosuppression. Once we understand each history, which is very challenging (and it's really the challenge that we're focusing on), we [can try] to individualize therapies for our transplant patients. That's why we're working in parallel at the Emory Transplant Center and trying to find a way (or the ways) to predict or individualize the transplant patient and then treat accordingly.

Questioner: I have a comment on the earlier enrichment discussion. The committee to revise the NRC Guide looked at a lot of this literature and that's why you'll see in the new Guide that enrichment is an experimental variable that should be controlled in animal studies. 


\section{Insights into the Brain and Aging: Session Introduction}

\section{Stuart Zola}

will begin with a quick introduction of the speakers. After I speak will be Dr. Nancy Hopkins, a geneticist in the Department of Biology at the Massachusetts Institute of Technology. Her work focuses on cloning vertebrate developmental genes by exploiting the zebrafish model. This work, over the years, has earned her nomination and acceptance into the American Academy of Arts and Sciences, the Institute of Medicine, and most recently the National Academy of Sciences. She will talk about genetic vulnerabilities associated with cancer and with aging.

Dr. Larry Young is William P. Timmie Professor in the Department of Psychiatry and Behavioral Sciences at Emory University. He is chief of the Division of Behavioral Neuroscience and Psychiatric Disorders at the Yerkes National Primate
Research Center at Emory. He will focus on understanding how genetic and cellular neurobiological mechanisms regulate complex social behavior. He uses a very interesting rodent species in this work. His title says depression, schizophrenia, and autism, but his major focus will be autism.

Dr. Erich Jarvis is at Howard Hughes Medical Institute, Department of Neurobiology at Duke University Medical Center. He studies the neurobiology of vocal communication with an emphasis on molecular pathways involved in perception and the production of learned vocalizations. In his work he uses an integrative approach that combines behavioral, anatomical, and molecular biological techniques, and he'll share some insights into brain development from animal vocalization studies.

\section{Challenges of an Aging Brain}

\section{Stuart Zola}

'm Director of the Yerkes National Primate Research Center. I'm also in the Department of Psychiatry and Behavioral Sciences at Emory University and a Senior Research Career Scientist with the VA Medical Center. My most important affiliation is actually [my membership in the] International Brotherhood of Magicians. I often use examples of magic to make points about the brain and brain function, and in this talk about the challenges of the aging brain I'll do just that with a simple example.

We can think of three pieces of rope-one short, one medium-sized, and one very long — as [representing] what happens to us as we age in terms of our memory, our cognitive functions as we are young and even middle-aged. We're doing pretty well as represented by this long length of rope. But as we get into our later years, our cognitive abilities aren't quite what they used to be, as in this shorter rope. Then the unfortunate reality is that as we get into our late decades - in our 70s, 80s, and 90s-we become vulnerable to a variety of aging-related conditions that can create problems for us in terms of our cognitive abilities and we begin to lose a lot of them, as [represented by] this very short rope. That's what happens, generally.

The goal of much [aging] research is to not have those kinds of changes take place over time: in middle age, later on, and in the very last part of our lives, all the rope lengths will be long and equal. Our cognitive abilities will be quite intact.

Figure 1 is meant to show the kind of interaction, the choreography, between basic and translational researchbetween research that we do in the lab, in this case research with nonhuman primates, and clinical applications of the research. The focus is particularly on the issue of cognitive changes over time.

The story starts in the 1950s in the clinic with the real case that changed all of our lives in terms of research and in terms of the beneficiaries of research: amnesic patient HM (who, by the way, died in 2008 and we've been able to look at his brain). He was the most prominent and most studied case in the history of the neuropsychology of memory and brain. He had amnesia, a memory impairment associated 
Figure 1 Translational research

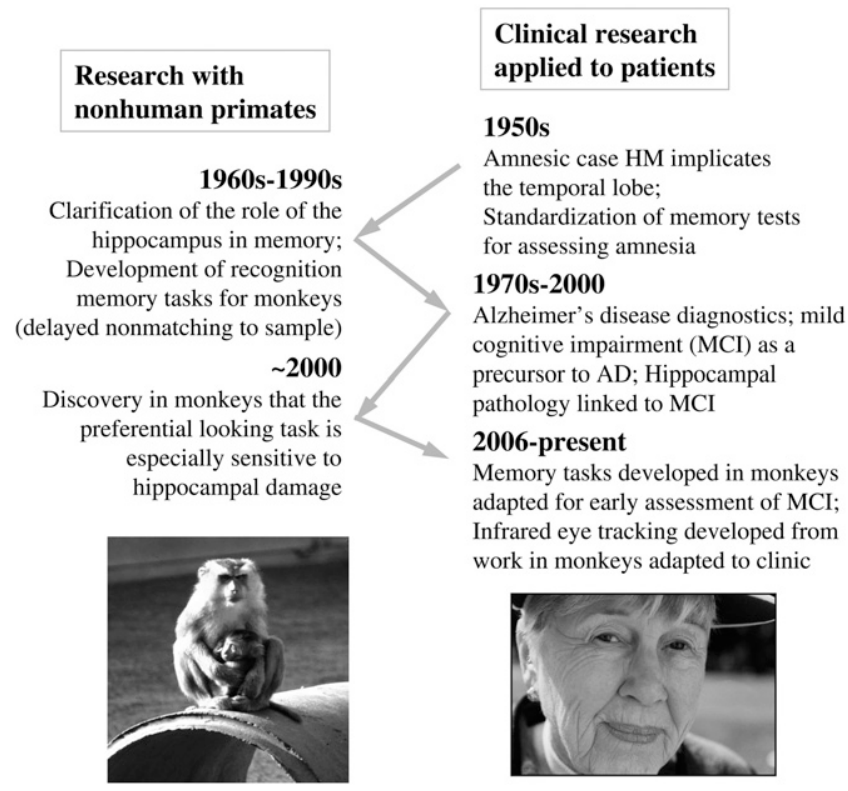

with a surgical procedure that was done to relieve temporal lobe seizures. In the $1950 \mathrm{~s}$, although there were medications available, none were effective. A surgical procedure was done with HM that was intended to relieve his seizureswhich it did to a great extent, but it left him with one of the most profound amnesias that we ever had the opportunity to study, and he was studied for over 50 years.

While this research was going on, a second development was the standardization of memory tests-that is, there developed a common agreement about the use of some standardized tests so we could test patients in Boston, Massachusetts, and patients in San Diego, California, using the same assessments. This development of standardized tests for assessing amnesia was an important additional step that came, in part, from the work with HM.

The work with HM in the 1950s stimulated a great deal of work in laboratories around the country with many animal models; I'm going to focus on nonhuman primates. One result of this research was the clarification of the role of the hippocampus in memory. There was a suspicion from HM that the hippocampus was critical but many studies during the 1960s through the 1990s substantially pinned this down and we began to understand how important the hippocampus was.

At the same time, we ran into exactly the same kind of problem in basic research that the clinicians did. We all had invented our own tasks to measure memory, and until we all began to use the same tests it was difficult to determine the roles of various brain structures because we didn't measure their function in the same way. The development of recognition memory tasks, and of one in particular called delayed nonmatching to sample, was a critical piece in the development of answers to this puzzle about memory. This really moved the field forward dramatically. A colleague of mine used to say that a neuropsychologist would rather use another neuropsychologist's toothbrush than use another neuropsychologist's test. That was the way the animal models field was too, until eventually we all started using the same tests. The delayed nonmatching to sample task was one of these; it allowed us to compare the performance of nonhuman primates across different laboratories and to compare directly the performance of humans with that of nonhuman primates.

That brings us back to the human realm, where, in the 1970s, Alzheimer's disease became very prominent. There was a meeting in 1975 that created a clear diagnosis for Alzheimer's disease but during the ensuing years another kind of condition was revealed, called mild cognitive impairment (MCI), that began to be understood from the early '90s to the present. Mild cognitive impairment is not dementia, it's not Alzheimer's disease, but it does seem for many individuals to be a precursor to Alzheimer's disease. Those who have MCI have a much higher risk than the general population of having Alzheimer's disease and it does, for some individuals, signal the potential onset of Alzheimer's disease.

Additionally, through brain imaging and other technologies, a link was established between disruption or deterioration of the hippocampus and mild cognitive impairment, and that brings us to the early 2000 s.

Looking again at monkeys, there was the development of a behavioral task that was an exquisitely sensitive test for looking at hippocampal function. It's called preferential looking or visual paired comparison. We'll see the relevance of that in just a moment; the important point is that the task is especially sensitive to hippocampal damage.

From about 2006 to the present, memory tasks were developed from work in monkeys and adapted directly to work in patients. We've added some other technologies like infrared eye tracking, also adapted from work in monkeys. Using these tasks and technologies with humans, we now have a way of identifying individuals who may be on a trajectory for Alzheimer's disease and we have the potential of intervening many years before the onset of the disease, a situation we did not have just a few years ago. The problem, as many of you know, is that there is currently no intervention that is effective. We can identify candidates but we cannot reverse the disease or change its course in a very significant way. That's still to come.

Deep in the temporal lobe of the brain is a critical structure called the hippocampus. There's one on each side of the brain in the lateral ventricles. When the hippocampus is damaged or when associated cortical regions that connect to it are damaged, memory impairment occurs. The kind of memory that is damaged is what is often referred to as everyday memory. We call it declarative memory - the ability to declare some aspect of memory. So if I ask what you had for breakfast this morning, you consciously recollect what you had, and you can declare it, you can tell me. The term declarative memory has been used for research with humans. Nonhuman primates can't talk, but we can ask them the same kinds of questions that we would ask our patients and we do that in some very clever ways. 
Figure 2 illustrates what we have discovered during 3 decades of research with nonhuman primates in terms of the memory system in the brain. This is the fundamental memory system in the temporal lobe. We know something about each of these structures, the hippocampal region and the cortical areas that I referred to earlier. All of these areas are critical for memory in different ways. They play different roles but we know a lot now about their interconnectivity and how they interact with the rest of the brain and why this region, especially together with these structures, is so important for memory. We have learned more about that from studies in nonhuman primates and other animals than from studies in humans. Work in humans has provided the ability to understand behaviorally what happens when memory goes awry, but studies in nonhuman primates and other animal models have made it possible to systematically investigate these structures and their roles. We've had to turn to work with animals to better understand that.

One of the ways we have done that is the classic delayed nonmatching to sample test. It's a simple idea. The animal first sees a single object, displaces the object, and gets a food reward. A door is then lowered, and, a varying interval of time later-from a few seconds to a few minutes or 10 minutesthe door is raised and the animal now sees two objects, the one it saw before and a novel one. In this task, the rule is simple: always go to the novel object. There's nothing under the object the animal has seen before if the animal displaces it. But if the animal displaces the novel object it gets another food reward. Animals have various preferences in terms of M\&Ms or raisins or peanuts or whatever they like-we try to figure that out — and they learn this rule very, very quickly. Once they learn the rule, we can change the delay interval

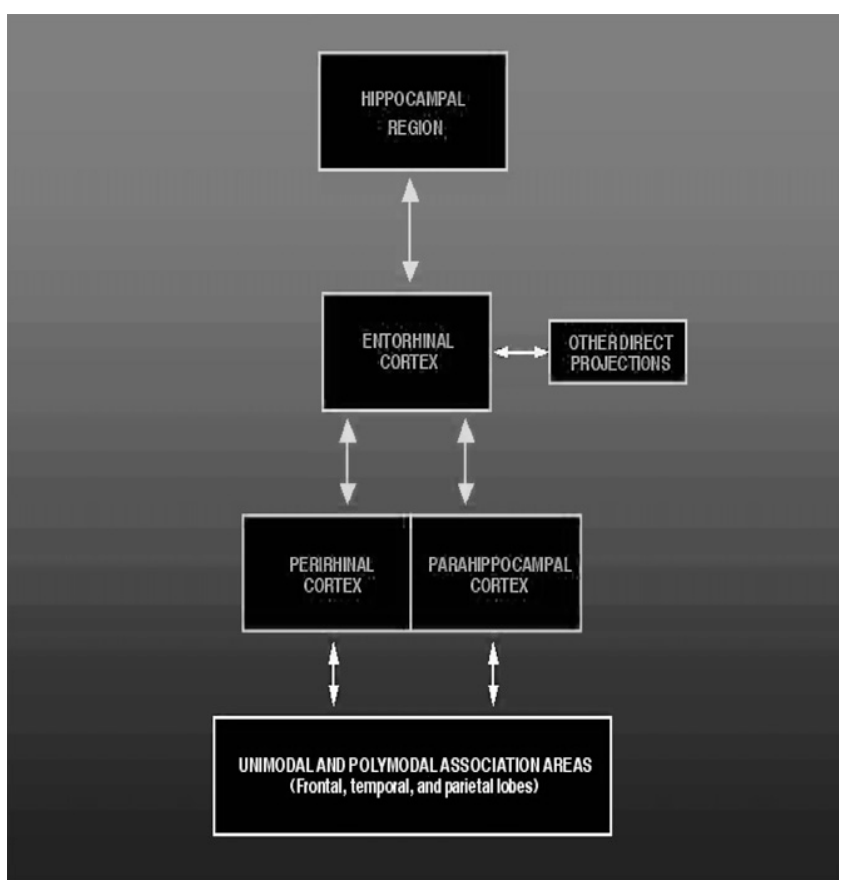

Figure 2 Memory system of the brain from the time we show them the sample to the time we ask them to make this choice.

We compare the performance of normal animals and animals with damage to the hippocampal system, the memory system of the brain, using delay intervals that are very short (10 seconds) to a delay of about 10 minutes. The result is simple: as the delays increase, normal animals, and you and I, forget on some of the trials. We're not sure which stimulus we saw before. But it turns out with damage to your hippocampus, your ability to do this task is disproportionally impaired. By 10 minutes, you're down to near chance performance. It's a two-choice test and you're just guessing at this point. The animal has no idea what the previous stimulus was. An interesting point here is that with very short delays, animals with hippocampal lesions that show impairment at longer delays perform quite normally here. There's no tax on memory at very short delays and you can do this part of the task with other parts of your brain-you don't need the hippocampus. You only need the hippocampus when there's a significant interval of time between the experience and the recall of that experience.

Once we discovered this in monkeys, the question was, How well did the findings with monkeys represent what we think is happening in humans? We moved to the clinic and tested patients who had hippocampal damage in real life. The setup looks very much the same as with nonhuman primates. There is an opaque door that can go up and down, and you can move a tray back and forth to set up the stimuli. The outcome of that work was essentially identical to what we discovered in nonhuman primates. Data from normal control individuals matched in age and education to the amnesic patients show that the latter, at 10 minutes, perform very close to chance, [whereas] at 10 seconds, a short interval, they perform normally. We discovered then that we could model in nonhuman primates the key characteristics that define memory problems and define amnesia in humans. That discovery set us on an important road.

I'm going to tell you about the other task, preferential looking, that was adapted from monkeys for humans. Interestingly, the task had been used to assess human infants many years ago. It involves a lot of technology.... The individual rests his chin in a chin holder (none of the equipment is attached to the individual) [and]... an infrared beam is reflected on a lens back at the eye. A video camera records movement of the eyes, which is also reflected back. All of that information is fed to a computer... The individual simply sits in front of a screen on which he sees stimuli exactly like those used in the task with nonhuman primates.

This technology allows us to determine, with remarkable precision, where the individual is looking on the stimuli and how long they're looking, what their saccades are, what their order of looking is. We can get a number of pieces of information.

I'll describe a close-up view of the familiarity part of the trial. In this situation, the individual looks at two identical stimuli for 10 seconds. The computer information is mapped to a diagram in which red circles represent the dwell time, 
how long the person looks at this particular spot, and red lines represent the saccades. Numbers in these circles represent the volume and sequence of where the individual looked. The blue $\mathrm{X}$ represents the first place the individual looked and then we can track the individual's scan path. If you add up the volumes, the individuals are looking at both stimuli about equally, that's what happens in the familiarity phase. You and I do exactly the same thing. Then the stimulus goes off the screen and 2 minutes later you see the original stimulus and a novel stimulus. In this situation with a normal individual, much of the looking is focused on the new stimulus, not the old stimulus. The individual looks at it a couple of times but really is exploring the new stimulus considerably. This is called preferential looking and it's what you and I do, and what 4- to 5-week-old infants and teenagers do, as well as nonhuman primates and rodents. Every species that has been tested does the same thing: they show preferential looking provided their brains are normal.

What happens, though, if you have damage to the memory system, to your hippocampus? After the familiarity phase, 2 minutes later when these slides go on, there is a difference. Individuals with hippocampal damage look equally at both stimuli as if they never saw the familiar stimulus before. The familiar stimulus is as equally interesting to them as the novel stimulus and that's the very simple assay of this test. It's an extraordinarily sensitive measure of recognition memory, and this all happens within a 2-minute interval. You don't need a very long time for this kind of forgetting to occur. It happens within 2 minutes. That turns out to be important for us in terms of testing patients because, as some of you know who work with patients, we don't typically have a big window of opportunity. Patients come into the clinic and have four or five or six different appointments and you don't have the luxury, as we do in the work with monkeys, of having the individual for 40 minutes or 1 hour. You have the patient for only 10 or 15 minutes, at the most, until they have to move on to the next appointment. You have to design a task that's sensitive enough to assay the kinds of difficulties in cognitive function that these individuals have and detect it very quickly and that is what this task allows us to do.

Figure 3A illustrates data points from all the patients we have tested in the past few years who had a diagnosis of Alzheimer's disease or MCI and normal controls matched in age and education to the MCI patients. Also shown are scores from a group of nonhuman primates who had damage to their hippocampus by surgery or by neurotoxic lesions. These animals model quite precisely what happens in advanced stages of Alzheimer's disease-their scores on the preferential looking test are very similar to the scores of the Alzheimer's patients. We supposed, then, that of the patients diagnosed with mild cognitive impairment, those with scores closer to the Alzheimer's patients and the monkeys with hippocampal damage might be at much higher risk than MCI individuals with scores outside that range. Moreover, control subjects with scores in the risk range might be at higher risk even though they are categorized as normal - that is, there is
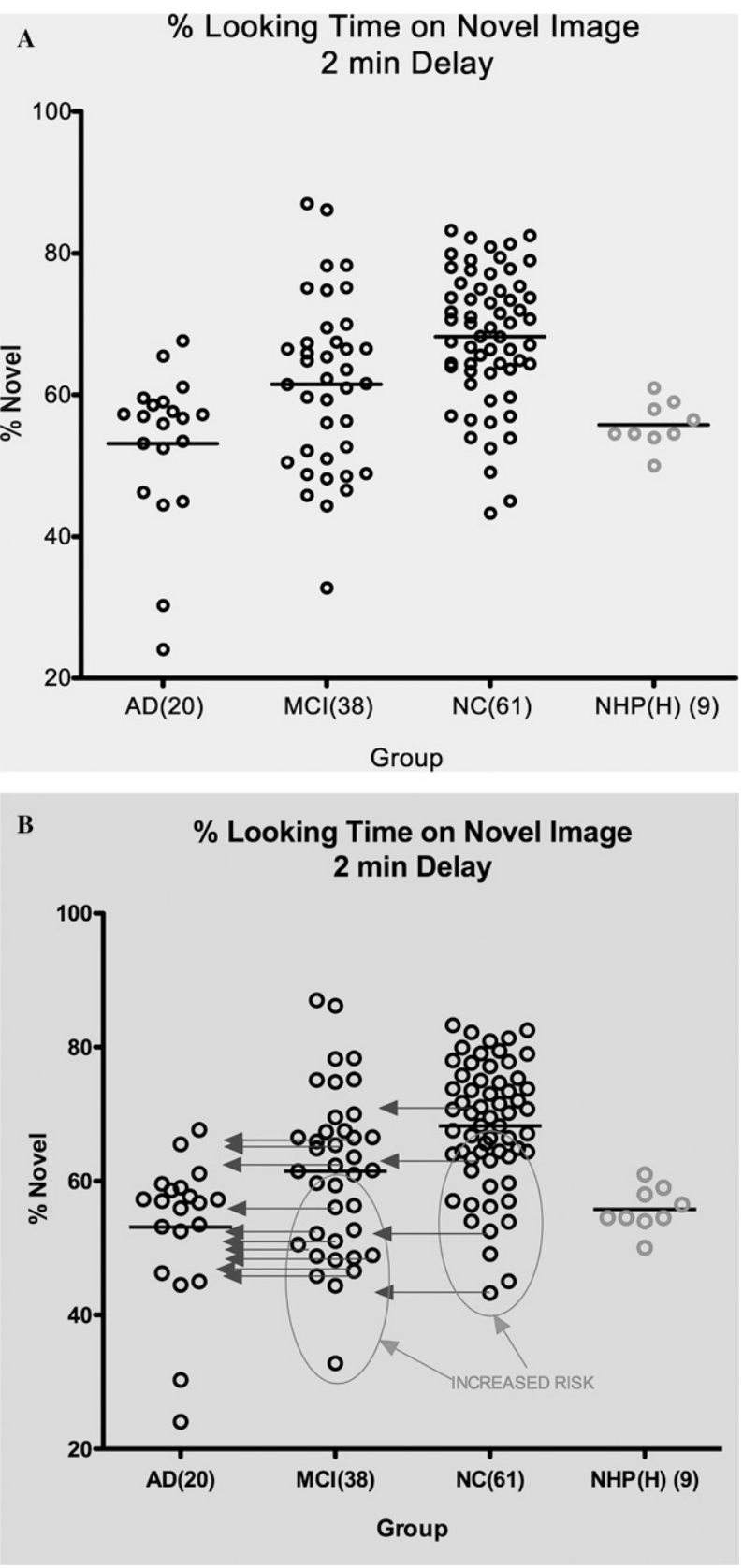

Figure 3A,B Percent looking time on novel image, 2-minute delay

no detection of any cognitive problems in any other assay. Nevertheless, they are more at risk, we believe, for deterioration of cognitive function. Over the last 4 years we have followed these patients. They are retested every year and it turns out that, of those individuals in the MCI group that we identified as being in the area of risk, most have been rediagnosed as having Alzheimer's disease (Figure 3B). In addition, several individuals in the normal group have been rediagnosed with mild cognitive impairment.

This development provides the promise of being able to detect individuals who are on a trajectory for Alzheimer's disease several years before they are diagnosed with the disease. It puts us in a position of being able to intervene sooner 
than later and that really, in a sense, is the Holy Grail for all of this work. Early diagnosis, whether we're talking about cancer or heart disease or Alzheimer's disease, puts us in a much better position for intervention, in this case because the brain isn't as compromised as it will be 3 or 4 or 5 years later. Whatever interventions we have may be more effective sooner than later in the course of the disease. So this is, in my view, a terrific story about how translational research has moved us from an understanding about brain function that we derived from research in nonhuman primates to a point where we can directly assess these kinds of things in the clinic. It has turned out to be remarkably informative for assessing patients in the clinic and predicting their trajectories.

Figure 4 shows what I call "the perplexing trajectories"perplexing not because we don't understand the trajectorieswe do-but [because] we don't know how to alter them. The top [line] is cognitive ability; it is declining. The other curve is neuropathology in the brain and it is growing. Most of us in this room are probably [at a point] where our cognitive abilities are quite intact and our neuropathology is minimal. Some others of us in this room probably have, or will have, mild cognitive impairment, difficulty in memory, but nothing else wrong. That is, you perform very well in everyday activities, you're not hospitalized, you're not institutionalized, but you have some memory slippage that is real. The difficulty is when these lines cross, and that is when the pathology becomes so great that it significantly affects our cognitive ability. Then we wind up in the domain of Alzheimer's disease and other dementias. Much of the focus in

Figure 4 The "perplexing trajectories" of Alzheimer's disease

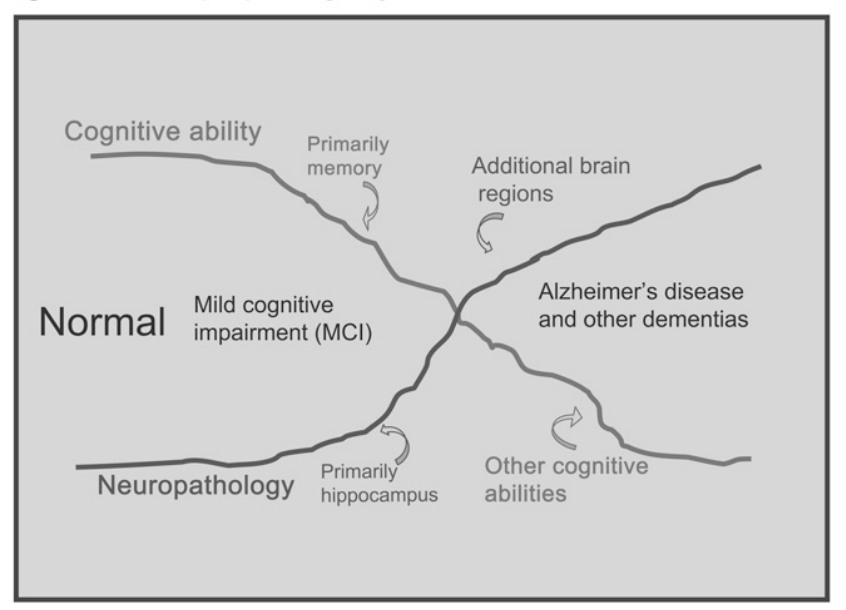

the field now is in the area of MCI because this is where we think we can prevent or slow the course. There isn't as much focus in the area of frank dementia because these individuals have brains that are in much worse shape and the likelihood of successful therapeutics is less.

We heard earlier about HIV/AIDS. These trajectories represent what happens in that field as well. Lots of people with HIV/AIDS are on HAART (highly active antiretroviral therapy), which helps control their disease. But the antivirals do not cross the blood-brain barrier, so although the individual's immune system functions well in late life, the individual's cognitive abilities do not, because the virus still has the potential to damage cognitive function. A critical challenge is how to systematically look at aging HIV/AIDS patients to understand how we might modify HAARTs so that they become more effective for these individuals who are living longer and longer.

I want to end with one additional point and bring us back to monkeys to describe a new technology that has been developed at the Yerkes Center, in particular, but at other research sites as well. We refer to it as "kiosk technology." This is a very clever development in which animals have microchips implanted below the skin in their wrists. Many of us have our pets microchipped so if they get lost they can be identified and we can be contacted about them. We use the same technology in nonhuman primates involved in behavioral research using the nonmatching task and others that have been automated with touchscreen devices in kiosks mounted around the outdoor compounds. Every time the animal works on a task it has to reach through an electronic circle to use the touchscreen and the microchip records the activity. Using this approach, we know each individual animal working on the task, how long they're working on it, what food reward they've gotten, and so forth. We can ask a number of kinds of questions about the behavior of these individuals and we can do it in outdoor social groups of 100 to 140 animals that live together in very large colonies. We can measure a variety of variables that may have some impact on aging and on cognitive function. We can look at age, sex, diet, and genetic factors. We can identify novel mechanisms of influence on cognitive decline, in terms of genetic sequence variation and gene expression. And we can study progressive diseases like Alzheimer's and SIV/HIV for which we have good animal models.

So we have come full circle from early work in humans and patient HM to some very sophisticated questions that we simply couldn't ask or answer in the era of HM. It's an exciting time for the field of neuroscience. 


\section{Genetic Vulnerabilities Associated with Cancer and Aging}

\section{Nancy Hopkins}

was asked to speak about the role of animal models in basic biomedical research, their importance for improving human health, why it's necessary to use animal models. We've heard quite a lot about this already but it was really fun to think about those questions, which I don't think about very often, honestly. I decided I would talk about two things that have intrigued me very much over the course of my career. One is something that Dr. Nakamura touched on, which is, Why is it that animal models have turned out to be far more powerful than we possibly could have imagined? Second, why is having more than one animal model often so important? I'd like to give an example of why from my own research, which today uses zebrafish to study cancer.

First, the question of why animal models have exceeded all expectations. I will give some historical examples that occurred over the past 40-plus years and hence during my scientific life.

I discovered molecular biology when I was a junior in college after hearing a single lecture by Jim Watson about DNA. The year was 1963, 10 years after the structure of DNA was published. Jim came into the classroom and talked about the central dogma and the structure of DNA. He made it clear to us, even to 19-year-olds, that DNA was the secret of life. He left no doubt that this was a universal thing, and that DNA and the central dogma was true of all organisms. This was the year the genetic code was actually being cracked (if you can imagine that that happened in the lifetime of living people!). Jim would come into class with codon assignments from Marshall Nuremberg's lab. It was astonishing - here was the triplet word for this amino acid or that amino acid.

It wasn't until a few years later, in 1967, when I was in graduate school, that we learned that the genetic code was actually universal. So the genetic code that was used by bacteria was also used by frogs and humans!

I don't know about you, but there are certain moments in one's life or career that are forever frozen into your memory. For example, where you were when you heard that President Kennedy had been shot. I think for scientists, we always remember where we were when we heard about a discovery that changed the way we looked at the world. That was one of them for me-that the genetic code was universal. It was completely thrilling. I think we just hadn't expected it.

Evolution didn't have to work that way, but it turned out it did, and thank heavens. That led to this comment by Jacques Monod, the great French scientist and Nobel laureate, that "What is true for E. coli is also true for the elephant." This was in 1967. What it meant to us then was that, yes, DNA was universally the stuff of life, the central dogma was universally true, and now, my heavens, the genetic code was even universal. But still, despite all this, beyond that it was not really imaginable to us how evolution would turn out to be less creative than we thought in terms of how it makes new things. It's been true over and over that all living things use very similar genes and mechanisms to carry out their biological activities. And this has been a profound gift to scientists.

Here are a few other examples that provided electrifying jolts in my scientific life. In the late 1970s, early ' 80 s, the discovery that genes needed for the early development of the fruit fly - these homeobox genes that were patterning the fly-were also present in humans was another thing that absolutely electrified and amazed us. In another example, I remember standing in Jim Watson's office in Cold Spring Harbor when he got a phone call from Bob Weinberg telling him that a human cancer gene-the first one that had been transfected and cloned-was the same as a gene that we already knew to be a cancer gene in mice and rats and chickens. Again, should we have known this? I don't think we could have imagined it and it was a stunning thing. You might have your own memorable moments-but these are facts that, the moment I heard them, changed my view of how the biological world works.

Another example comes from studies of the genetics of aging_-particularly the work of Cynthia Kenyon at UCSF who studies lifespan extension using nematodes. She discovered a set of genes that when mutated cause the worm to live longer. Incredibly, it has turned out that some of those genes also extend lifespan in mice. I wrote to Cynthia yesterday evening because I wasn't sure where the data stood on humans. She said that variants of some of the genes she identified are now linked to extreme longevity in humans! That the same genes that expand lifespan in nematodes should do it in mice, and possibly also in humans, is astonishing.

What's remarkable about the genes for aging is that when you push the lifespan back by mutating these genes, you don't extend old age, you extend early and middle age. You delay the onset of the diseases of old age, they occur later when you make this genetic change. So it's not science fiction to think that if you could find drugs that act similarly to the way these genes are acting you could in fact treat people and push back all the diseases of old age. It would surely be a most efficient way to treat the diseases of old age. 
These are some of the reasons biomedical research has advanced as quickly as it has. When I took that biology class as a junior in college, we could foresee that one day you would know the genetic basis of cancer but we thought it would take about 200 years. Instead, it's taken only about 40 to 50 years. I attribute this to the astonishing conservation of gene function across vast evolutionary distances. This has made animals far more useful as research tools for understanding human biology than we really could've imagined and it has accelerated biomedical research by decadespossibly by centuries.

If the genes and their functions are so well preserved, why can't we do cancer research in yeast or flies? Why do you need mice? If mice are great, which they are, for cancer research, why do you need fish, which is what I'm going to talk about now? There are substantive reasons and they're probably obvious to this particular group of people. One is, of course, that not all processes happen in yeast or flies. The other is that despite the astonishing molecular and cellular similarities across species, as you know there are also differences. Sometimes those differences are extremely helpful in illuminating the biological process you're interested in. But unfortunately it also means that almost no animal is a perfect model for a human disease and often you need more than one if you're going to study all the aspects of a problem.

Let me turn now to the organism that I work on, the zebrafish, to illustrate these two points: that you need to have the animal that is perfect for the problem at hand and that sometimes having an additional model can help you to understand aspects of a problem that was intractable.

The zebrafish is well known as a superb organism in which to study early vertebrate development. I'm going to talk today about how it's also becoming an organism that is very interesting for the study of cancer. This is something I was slow to realize.

First, why was the zebrafish so superb for the study of early vertebrate development? The zebrafish was chosen from among the possible vertebrates for finding the genes that are responsible for the development of vertebrate structures. The choice was made by George Streisinger, a professor in Eugene, Oregon, after lots of trial and error to select the right organism.

One thing you had to have if you were going to do genetics of development was an organism that is easy to maintain in the lab because to do a large genetic screen you have to be able to maintain thousands of animals. The fruit fly screens that looked for the genes needed for development involved tens of thousands of families of flies and screened through literally millions of embryos to find the ones with genetic defects of interest. So the animal has to be able to mate a lot on demand and mate all year round in a laboratory, and they have to lay a lot of eggs because you want to get Mendelian ratios. If you don't get a lot of progeny, you don't have enough to do the counts. And finally, if you're going to screen for defects in early development, the process you are screening for has to be visible and accessible. The zebrafish happens to have an incredibly beautiful embryo that's transparent and develops very rapidly outside the mother, so you can watch it and you can see things go wrong if there's a mutation.

Figure 1 shows the first 5 days in the life of a zebrafish. In the top row is the four-cell stage, 1 hour after fertilization, with the yoke and the four cells on top. They cleave very rapidly - every 20 minutes - to produce a few thousand cells that sit atop the yoke. Then these cells are pulled down over the yoke in the process of epiboly and begin gastrulation. The cell sheet moves over the yoke until it completely envelops it, and very complex cell movements occur within the sheet. The morning after the fish laid eggs, you see this beautiful little creature, which is inside a chorion (which has been removed for this picture but is also transparent). You're looking right through the chorion at this animal that is moving its tail vigorously before 24 hours of age. By 5 days of age this creature gets up and swims away and you have to start feeding it. By then of course it has a beating heart and functioning organ systems.

The idea was that you could do very large genetic screens and identify essentially all the genes that are necessary to make this picture happen. When I say necessary I mean if you took out one gene at a time, which ones would have an effect on this picture so that you could see the effect in a dissecting microscope. First you mutagenize the genome, next you do appropriate outcrosses and inbreeding, and then you examine millions of embryos to look for the ones that carry

\section{Figure 1 Zebrafish development from hour 1 to day 5}

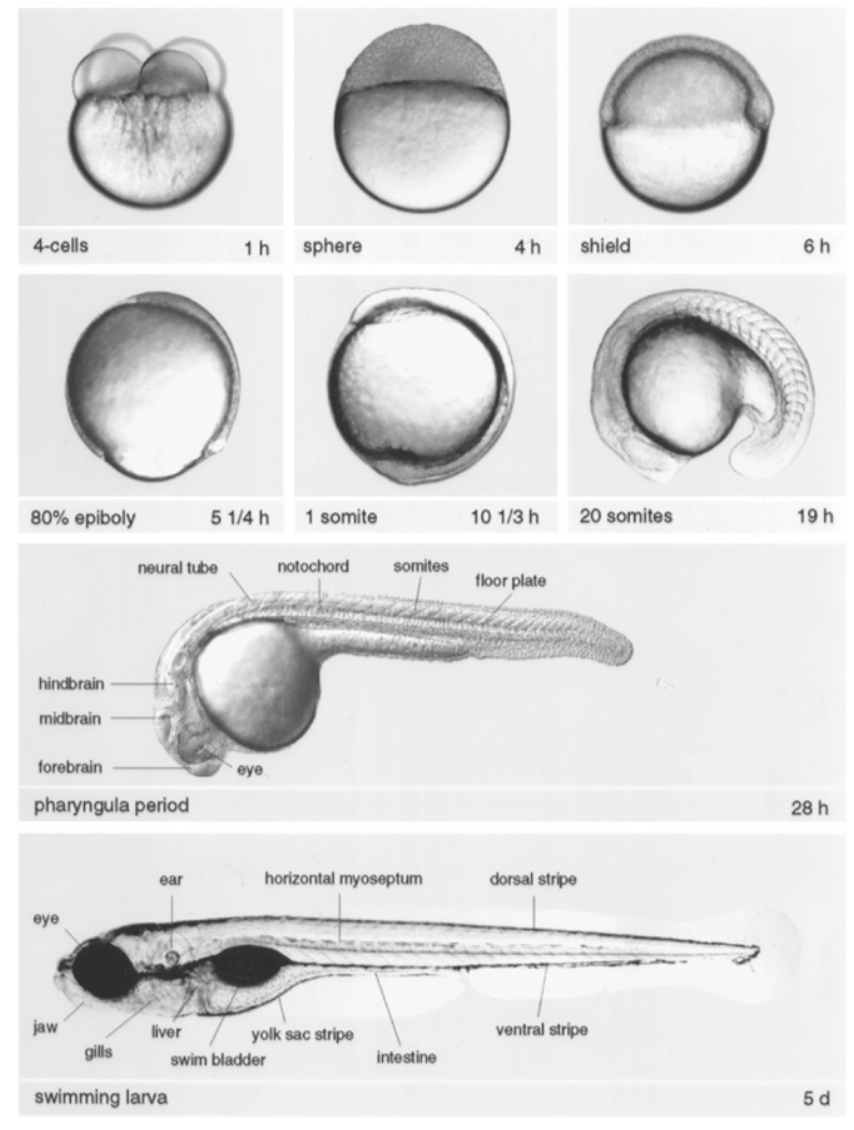


developmental defects. I became captivated by this capability of the fish and when I returned to MIT I worked on developing technology to do an insertional mutagenesis screen where you could bombard the genome with exogenous DNA (or in this case a virus) and it would mutate the genome and then you could rapidly clone all the mutated genes. It was a very ambitious project and it worked, which was unpredictable because you never know, when you go to develop technologies, whether it's going to work. We developed this insertional mutagenesis method and mutagenized the genome of the fish, bred insertions to homozygosity, screened for mutations, isolated about 550 mutant lines of fish with defects in genes required for early development, and cloned almost all the mutated genes.

What did we find from this experiment? We discovered that we had identified about a quarter of the genes that were essential for the picture of early development to take place. We found that the total number of genes is actually very small - there are only about 1400 genes and, if you take them away one at a time, you'll see something go wrong in the picture at the level of a dissecting microscope. Now perhaps we should have known this in advance, after what I told you before about evolution and the uniformity of life, but we didn't. I don't know if we could've guessed but it turns out that the number of essential genes is, again, highly conserved through evolution and so are genes themselves. Of those 1400 genes, 1000 of them are essential to make the cells that the embryo is made of, and the other 400 are needed to pattern those cells and to cause them to form the different structures that make up the embryo.

Remarkably, the number of essential genes in yeast is also 1000 . The number in flies is 1000 . The number of embryonic genes in the fly is a couple of hundred and if you look at what these genes actually are, once again you find that they're conserved through evolution. So the fact of being an essential gene is itself evolutionarily conserved. There's a tiny number of such genes. To make an adult animal takes a few thousand more.

This research came along at the time of the sequencing of animal genomes. The numbers of genes in the genome kept shrinking and we said, "It's about time because there aren't that many essential genes." The gene numbers for humans were dropping from about 100,000 predicted genes to about 20,000. That's three or four times the number of essential genes; the reason for this is unknown but perhaps very interesting. Suffice it to say that, as a result of the screen we did, we had all these mutant lines of fish and of course we gave them to people and many people came and screened the collection because every time you screened it, you were screening $25 \%$ of genes that could be found that affected the early development of the embryo. People who worked on many different organs came to the lab and took away mutants, which is a wonderful thing. The purpose of doing screens is to give away most of what you make.

So we were carrying these many lines in the laboratory and we hadn't thought about the fact that some mutations, which are lethal as homozygotes and cause embryonic defects, can have phenotypes as heterozygotes. It turned out that some of these lines of fish developed tumors and had early mortality. You had to be careful and watch them or you might lose the line. For a long time we had to carry 550 lines of fish live because we didn't have them frozen down yet. Now the collection has been preserved by freezing sperm from the mutant lines (frozen sperm is kept at the stock center in Eugene, Oregon).

Some lines appeared to get cancer. We had a veterinary pathologist look at these animals. He said they had something called malignant peripheral nerve sheath tumors (MPNSTs). We were intrigued by that and had all the mutant genes cloned so we could quickly find out which were the genes that were predisposing to cancer. It turned out that about $5 \%$ of the genes we had found predisposed to cancer. A couple of them were already known to be oncogenes in mice and humans. But another group of them were a new class of haploinsufficient tumor suppressors. We had discovered a new category of cancer genes in the fish. So I asked myself, Do you want to work on cancer in zebrafish? I used to be a retrovirologist, but I had left the cancer field, gone into early development using the fish, and now here I was in the cancer field again. I was a little skeptical; I thought, Is the fish really the answer to cancer? Could it contribute something?

I have to thank some colleagues at Harvard Medical School: the labs of Len Zon and Tom Look. While we were backing into the use of zebrafish to work on cancer, they were deliberately setting up to turn the zebrafish into a serious model organism to study cancer. Without that, it would have been difficult for us to take up the study of cancer in fish. They took it on as a major effort and what they found was [that] if you took oncogenes that were known to be cancer genes in the mouse and human, or knocked out known tumor suppressor genes, these all caused cancer in fish just as they did in mice and humans. You'd think at this point that everybody would say, "Of course they do." You would be astonished at how frequently you send a paper to be reviewed or write a grant and a reviewer writes, "But why would you think the same genes cause cancer in fish that cause cancer in mice and humans?" Despite 40 years of research showing over and over again that evolution worked, you still have to convince people over and over again. So, many thanks to Len Zon and Tom Look, who have done studies showing that indeed the same cancer genes work in the zebrafish that work in mice and humans.

When you look at tumors in fish they look very similar to the human counterparts, and gene expression is also very similar. This is extremely encouraging. But again, I think you still have to ask, What does the zebrafish have to offer as a cancer model that the mouse can't provide? I'll finish with a short story from our own lab, which I think shows why having a single animal model is seldom sufficient to study a biological problem and why the fish has unique contributions to make to the study of human cancer. 
We've been using the fish to look at an interesting issue in human cancer: aneuploidy. We became interested in knowing whether zebrafish cancers are aneuploid (i.e., they have a nondiploid number of chromosomes). Human cancers are often highly aneuploid, and this is particularly true of solid tumors and late-stage tumors. This fact has been known for over a century... Not only are human cancers often highly aneuploid but with such cancers the genome is often a mess. The significance of this has remained elusive but what is known is that in human cancers there is cell-to-cell variability in chromosome number-a tumor is really a collection of cells with different chromosome numbers. Some chromosomes or arms are preferentially overretained in a tumor and some are underrepresented. The significance of this fact is not understood.

It's also true that certain tumor types have certain chromosomes increased in number and others preferentially reduced. In addition, highly aneuploid tumors usually also have translocations, inversions, focal amplifications, deletions, and single base changes-lots of genetic changes. The government's about to spend a vast sum of money to try to take a large number of human tumors and identify all the genetic changes in those tumors and try to see, among the different changes, which are the significant ones and whether it's possible to identify "signatures" of each tumor, the drivers for that tumor, [in order to] develop combinations of drugs so you can treat those tumors. Imagine the undertaking when you see the complexity of these genomes.
Needless to say, aneuploidy of human cancer has been very difficult to study. Part of the problem is that many mouse cancer models are not highly aneuploid the way human cancers are. There may be aneuploidy but not to the extent you see with the human models. People have had to deliberately generate genetically modified mouse models with genome instability built into them to increase aneuploidy [to resemble] that seen in human cancers so they can use the mouse as a model to study this type of change in human cancers. But at least some fish tumors are highly aneuploid and look like human tumors in this regard. Figure 2 shows the karyotype of a normal zebrafish cell and that of a cell from a tumor. The graphs (Figure 2C,F) show a count of the number of chromosomes per cell, counting 100 cells from the tumor. You see that the numbers are all over the place. The diploid number is 50 but in a tumor the number of chromosomes ranges from 40 to 100 per cell and each cell is different. It's quite alarming when you look at this to ask, What is a cancer genome?

Figure 3 shows a summary of data from many cases of human MPNSTs and from zebrafish. On top are the 25 chromosomes of the fish and on the bottom the 23 chromosomes of the human. You see that some chromosomes are overrepresented and some underrepresented.... Could you use this information from fish to ask, When chromosomes are up is there something about the genes on those chromosomes that is common? Let's suppose you had done this experiment in mice and got the same result. You would find that most of the genes that lie on a chromosome that's up in the human, if

Figure 2 Fish tumors are aneuploid like human cancers

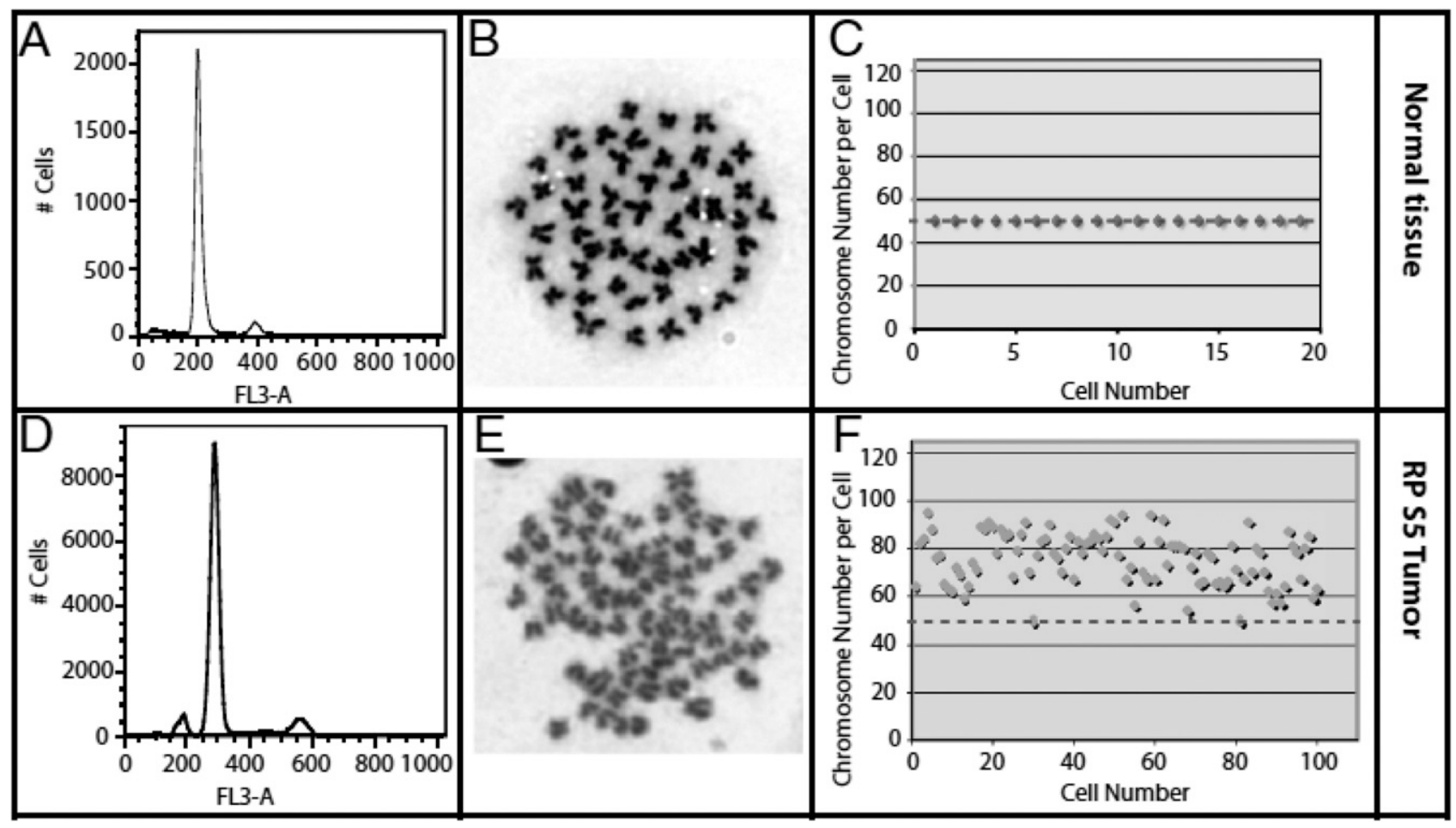



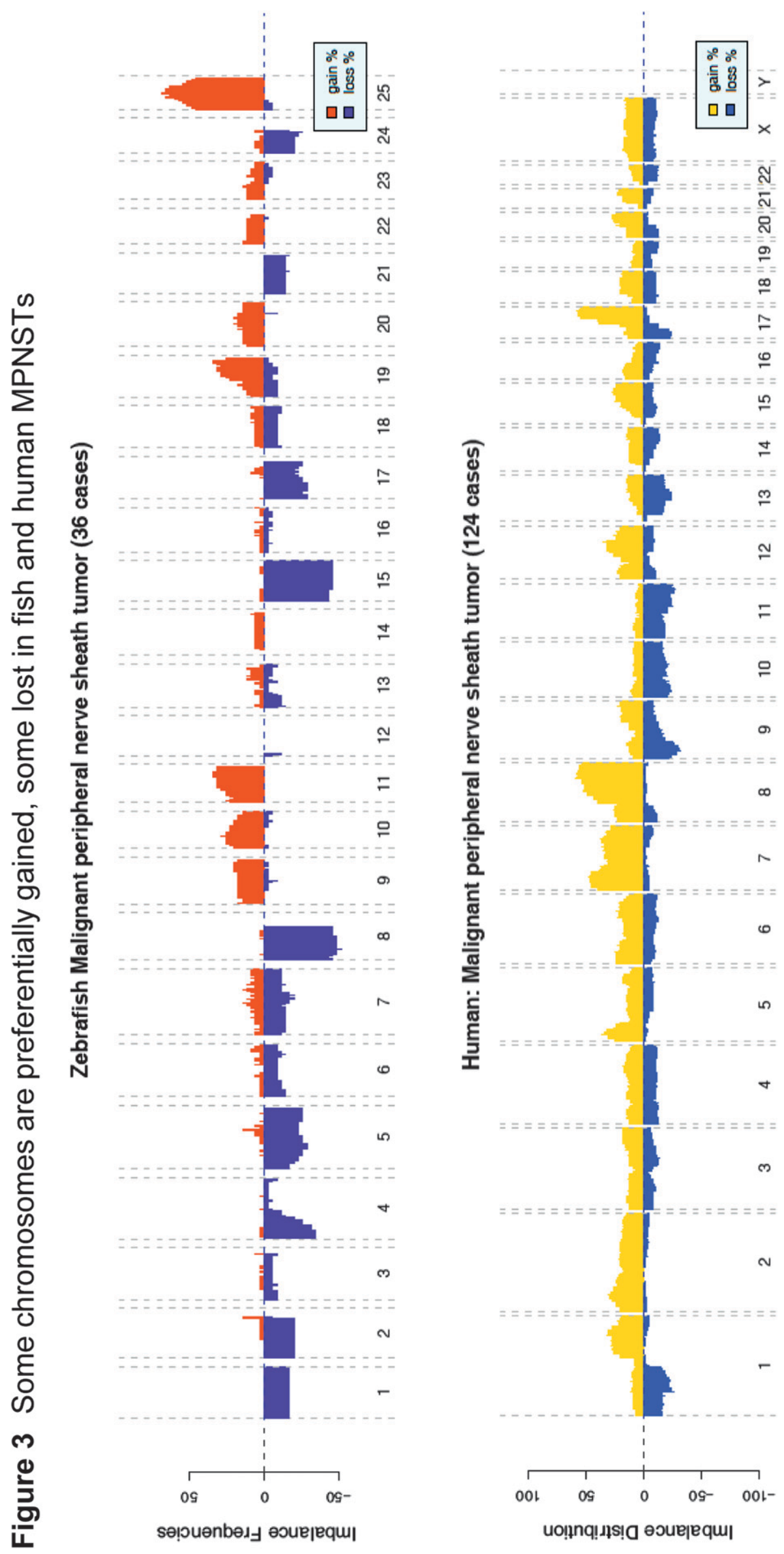


\section{Figure 4 Human-fish versus human-mouse chromosome pairs}

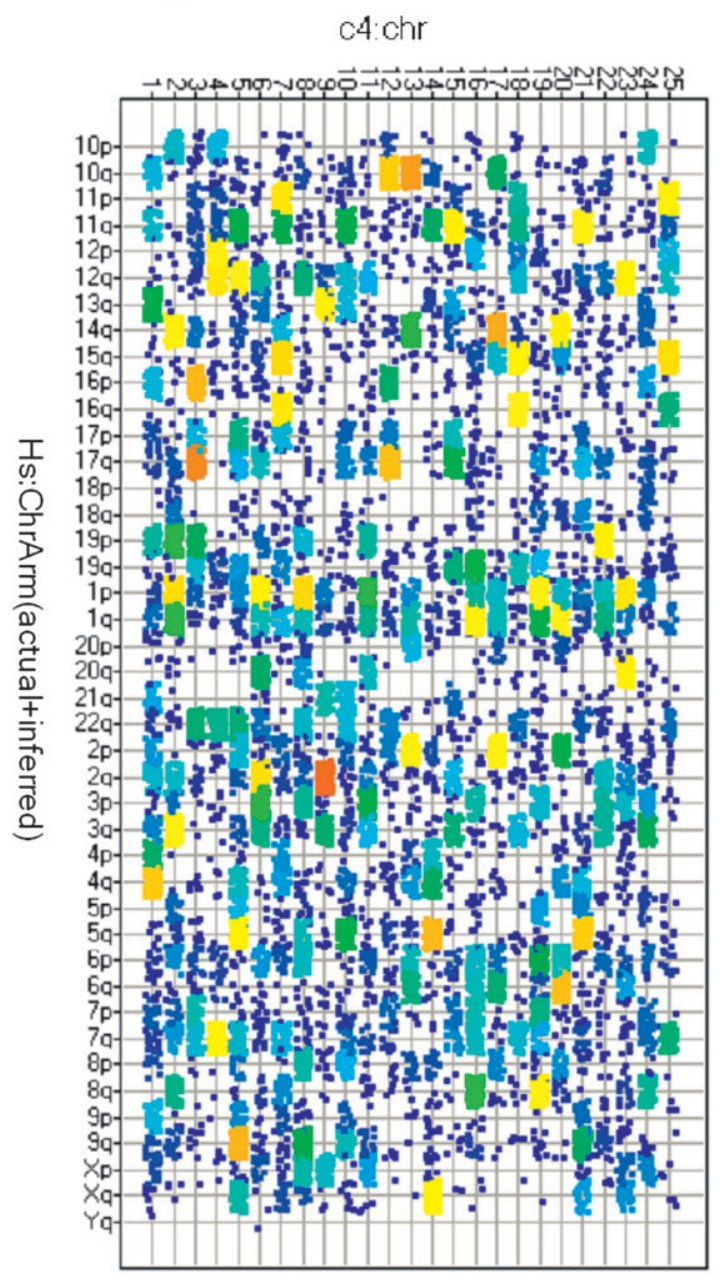

that chromosome were up in a mouse, would share most of the genes because those organisms are pretty close evolutionarily and the genes tend to lie on the same or corresponding chromosomes.

But in the fish, because humans and fish are far apart evolutionarily, the genes have been scrambled. When you have chromosomes that are up, if you ask "How many genes are shared between this chromosome and this one?" the answer is going to be "a small fraction of the number you'd find if you had compared it to the mouse." That's shown in Figure 4, a comparison of orthologous pairs of genes on all human and fish chromosomes versus all human and mouse chromosomes. The gene order has been shattered and the genes are scrambled among the chromosomes. Where you have now increased numbers of certain chromosomes, you've enriched for the genes that are on them in both fish and human, thus reducing that number of candidates by over a factor of 10. So evolution was on your side in this case by creating difference instead of similarity that should help you to find the important genes that contribute to cancer as a result of chromosome overrepresentation.

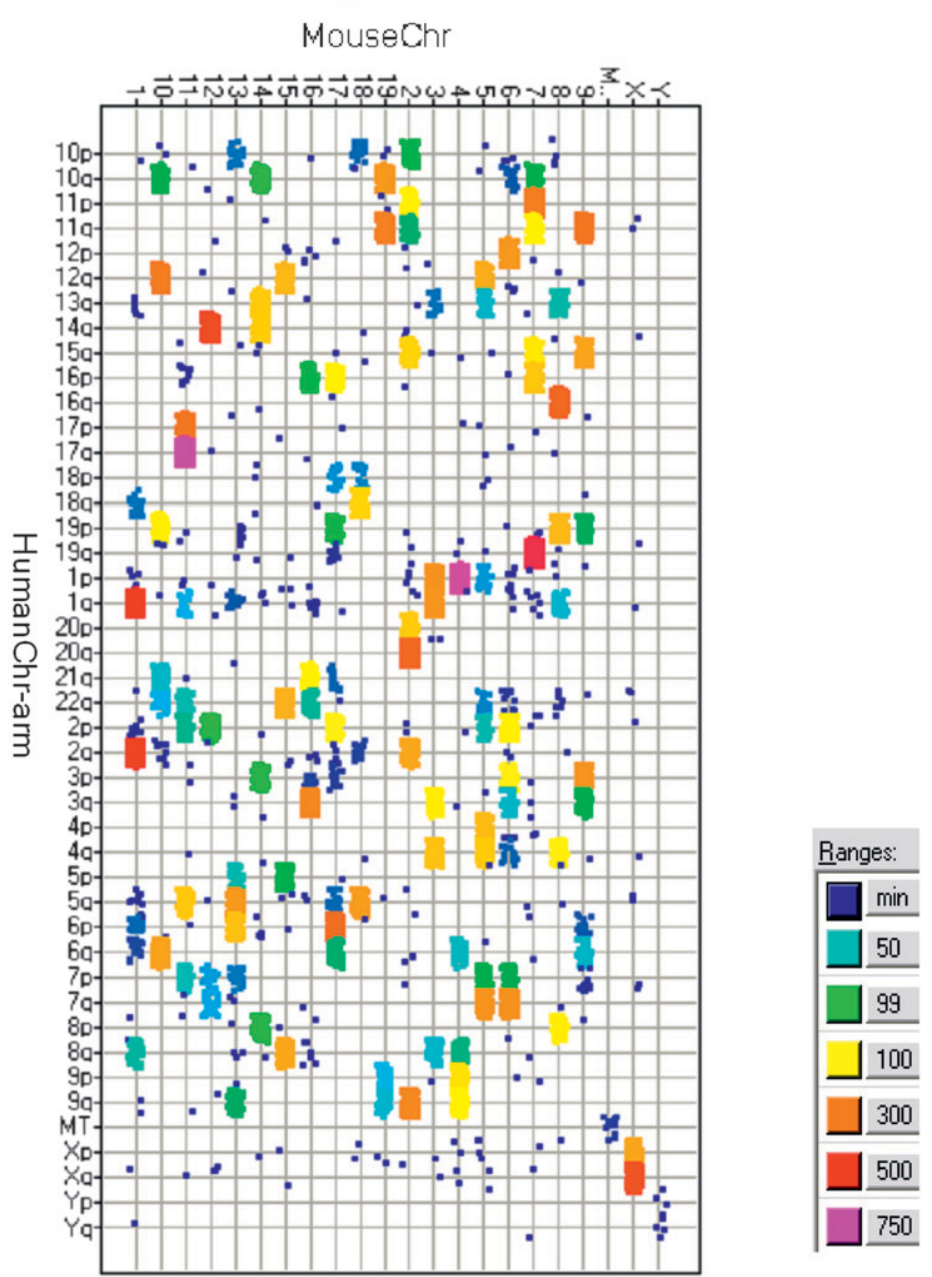

In summary, the extraordinary biological similarities of diverse species make animal models more valuable for biomedical research than we could have hoped for. But differences between species are also valuable, and for this reason you usually need more than one model organism.

In closing I would like to say that one of the things that really intrigues me is the idea of one day being able to get rid of animal models entirely. That is an interesting idea. I'm all for it in theory, I think it's a great idea. I'm going to go home and think about this a lot. I also think you have to push people in that direction. You have to be the ones who push on the basic scientists to do that because we're not thinking that way and it pushes us to think in a new and interesting way. I urge you, those of you who do this, to be aggressive and pushy with basic scientists who push back. We're thinking about something else, but it's fascinating and important to think that we could keep reducing the use of animals and also always try to use the simplest organism, the one that's lowest on the evolutionary scale, when possible, for the particular problem at hand rather than the one you just happen to have in the lab. 


\title{
Toward a Better Understanding of Depression, Schizophrenia, and Autism
}

\author{
Larry Young
}

will discuss the work that my laboratory has been doing to identify better strategies for treating social deficits in psychiatric disorders such as autism. The title of my talk is actually "How Monogamous Rodents Can Help in Drug Discovery for Autism." You may be wondering, What in the world does a monogamous rodent have to do with autism? That's actually a very important point that we've heard several times in talks here: sometimes when you're doing basic science you make connections that you never knew existed. The story I'm going to tell is an example of that kind of connection.

Autism spectrum disorder is a class of disorders characterized by three main, core phenotypes. The one we are most familiar with is the deficit in social engagement and social reciprocity. There are also deficits in communicationindividuals with autism often have difficulty in communication or show language delay. And individuals with autism spectrum disorder often have highly repetitive and ritualistic kinds of behaviors.

Autism has gained a lot of attention in the media in the last several years. One of the things people have been discussing is that perhaps there is an increase in the prevalence of autism spectrum disorders. Actually I don't think that there is a large increase in prevalence, but rather an increase in understanding and diagnosis of the disorder. The latest figures from the state of Georgia [indicate that] about 1 in 100 individuals can be diagnosed with autism spectrum disorder. The figure from the CDC for national prevalence is about 1 in 166 .

One of the interesting features of autism that I think is important to try to understand is that there is a skewed sex ratio for the disorder-four to one males to females. In Asperger's syndrome, which is a milder and higher-functioning type of autism spectrum disorder, it's eight males to one female.

One of the difficulties in trying to figure out how we might go about treating autism spectrum disorder is that it seems to have multiple roots. It's a collection of syndromes, as I mentioned. There are many single genes that have been identified to contribute to risk factors for autism, so there is not a single gene that causes it. There are some forms of autism that a single gene does contribute to, but there are many, many genes that seem to contribute, rearrangements in chromosomes, as well as some environmental factors that we don't quite understand. This creates a real challenge in trying to understand how you might develop treatments for the disorder because it's not one disorder [but] a collection of disorders with a common phenotype.
Scientists have been trying to figure out how to develop animal models - and I have to say I think this is a case where it's absolutely essential to have animal models, because we're never going to be able to understand the etiology of this disorder and develop new therapies without having some animal models to be able to investigate how we might be able to treat it. One of the more common ways to use animal models for autism is to use mice, for example, that have mutations in genes that have been identified in autistic individuals as contributing to autism, and then study how that gene affects neural activity or behavior. A great example of that is the FMRl gene, which is responsible for Fragile $\mathrm{X}$ syndrome, where studying animal models with mutations that give rise to this disorder has led to potential treatments for the disorder. Other investigators look at how environmental insults might contribute to brain development-for example, certain drugs that have been linked to an increase in autism spectrum disorder.

My lab has been taking a little bit different approach. We've been focusing on one of the core phenotypes-social behavior - to try to understand the social brain. My philosophy is that if you can understand the basic neurobiology of the social brain, you might have a better handle on how these myriad different genes and environmental insults might cause defects in the social brain and may indeed be able to improve social functioning in autistic individuals.

When I got involved in research, I wasn't thinking about autism at all. I was a young postdoc and mostly interested in basic, fundamental neuroscience to understand how the brain works. I wasn't thinking about treating anyone. I became interested in understanding the biology of social relationships. How can we understand what makes us want to engage in relationship with others? If you look at humans, we are a little different from many other species in terms of social behavior, we have different kinds of social relationships. Some of them are evolutionarily very ancient, like the mother-infant bond. In virtually every mammalian species there's a relationship between the mother and the infant, it's an ancient mechanism. But humans also have a relationship that forms between the father and the offspring in many cases and also a relationship that forms between the two parents, which I will refer to as a pair bond. That is something that is difficult to study in most animal species. If you're interested in studying the biology of that type of bond you can't really study it in humans, you have to develop an animal model.

Mice and rats aren't particularly good animal models for studying social bonding because they don't form these kinds 
of complex relationships. I'm going to tell you about an animal model that is ideal for this type of study, but before I do I'm going to make a couple of points that I want you to keep in mind. One is that it's critical to choose an animal model that is ideally suited for the questions you're going to ask. Mice and rats are great models for many questions, but they're not ideally suited for everything you want to study. Also, keep in mind that sometimes, even if you can't imagine how, basic animal research may lead directly to a clinical outcome - treatment of humans - and you never know where those links are going to be made. This story is a great example of that.

The animals I study are prairie voles. These are wild animals that live in the northern Midwest of the United States. They are different from many other laboratory animals in the sense that they are highly social. Mice are highly social as well, but prairie voles are very highly social and also form social bonds between sexual partners, or pair bonds. If you put a male and female together, in the wild or in the laboratory, they form a bond that will last a lifetime. The male spends just as much time interacting with the offspring as does the female. So what I began to do, when I began working with these, was try to understand the brain mechanisms that lead to that bond formation.

When you're doing behavioral research you have to have a good behavioral assay to be able to answer the question you are interested in asking. Sue Carter's group developed a behavioral task to ask if these animals have formed a bond with their partner or not.

In a laboratory test called the partner preference test, we put a male and a female together for a defined cohabitation period, we allow them to mate or we prevent them from mating, give them various drugs to affect brain receptors. Then we separate them, place them in the partner preference arena (Figure 1A), and test whether they have bonded. If testing the female bond, we take the male partner and tether him with a little collar around his neck to one side of a three-chambered arena; he can move around his chamber but not into the others. We then take a novel male that she's never seen before and tether him on the other side so that he can move around that chamber but not into the others. Then we drop the female in the middle and watch to see who she spends her time with. If these animals have mated and been together for at lease 12 hours, the females typically will have bonded to their partner and will spend more than twice as much time with the partner as with the stranger. They'll huddle with each other and groom each other. This is how we determine whether a pair bond is formed. We can perform the same test of the male bond by tethering the partner female and a novel female in two chambers.

Now I'm going to tell you about a molecule that we know plays a critical role in this pair bond formation: oxytocin. It's a neuropeptide that is produced in the hypothalamus; you probably know about its role in the periphery. It's released from the pituitary at the initiation of labor and stimulates uterus contractions, so it plays an important role in promoting birth. Once the baby is born, it continues to be secreted, and when the baby is suckling the neural connections from the breast to the hypothalamus cause those neurons to fire again and cause oxytocins to be released, which causes smooth muscle contraction in the breast to stimulate milk ejections. These roles of oxytocin have been well understood for more than 50 years (Burbach et al. 2006).

Much more recently people started doing behavioral studies to ask if this molecule, which is so critical for giving birth and nurturing the offspring, might be stimulating some of the behavioral changes associated with giving birth. If you put a rat pup in the cage with a virgin female rat, that female rat does not like the pup-she finds it annoying and actually will try to bury or get away from it. But when a female rat goes through her pregnancy, toward the end of that pregnancy something changes in her brain, so if you put a pup in her cage she doesn't try to avoid it, she tries to nurture and lick and groom it. Female rats will even cross an electrified grid to get access to a pup. So a tremendous change happens in the brain that changes their motivation to nurture. Part of that change we now know is due to oxytocin: you can take a virgin female rat, inject oxytocin into her brain, and put a pup in her cage and she will nurture that pup (see Ross and Young 2009 for a review).

Rats don't really bond with individual babies because they have lots of babies in the nest and, in the wild, no other female is going to come drop a baby in their nest. So they don't need to have a bond to their particular offspring. But sheep are a different story. Sheep live in herds and there's a breeding season, when lots of females are giving birth at the same time. When a ewe gives birth, within just a few minutes the lamb is able to walk around. So the ewe not only has to decide that she is attracted to lambs and wants to nurture

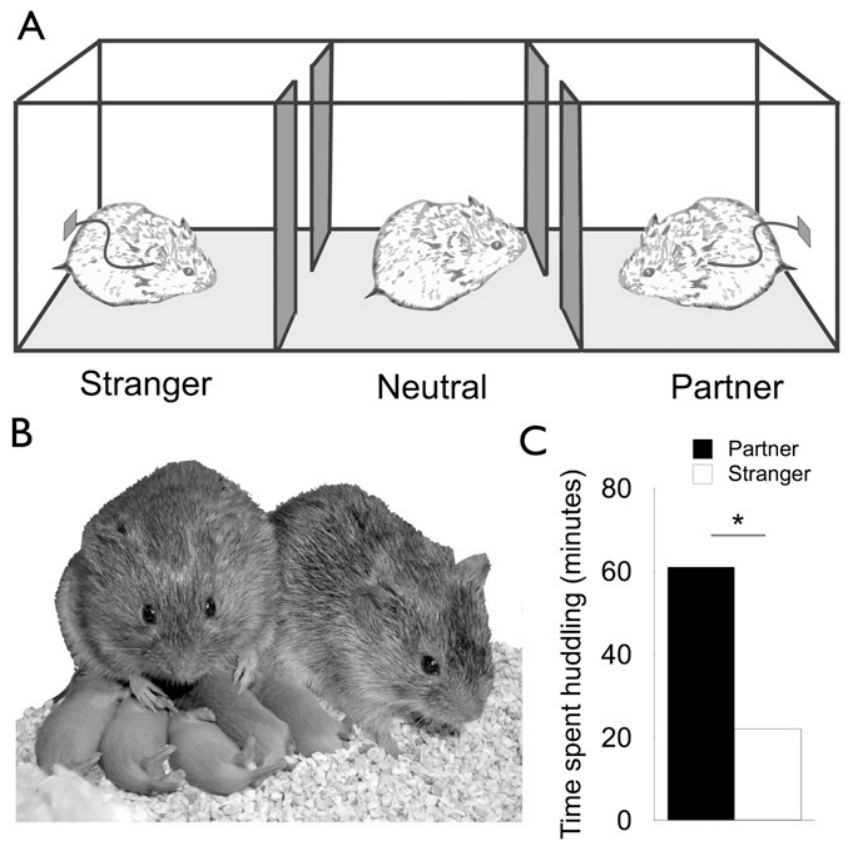

Figure 1 Use of the partner preference arena (A) to assess pair bonding in prairie voles $(\mathrm{B})$ through $(\mathrm{C})$ measurement of time spent huddling with a familiar versus unfamiliar vole of the opposite sex. 
them, but she has to bond with her particular lamb and butt away other lambs. That bonding process we now know is due to oxytocin. You can take a ewe who is not giving birth but has been estrogen- and progesterone-primed, show her a lamb and she will not nurture it. But if you inject her brain with oxytocin she will bond with that lamb and nurture it. These examples illustrate the powerful effects of this neuropeptide on behavior.

Now I will turn back to voles and the pair bonding process. Given that oxytocin is involved in maternal bonding, Sue Carter and Tom Insel back in the early 1990s wondered whether it might be responsible for the female prairie vole bonding to the male prairie vole (Williams et al. 1994). They did a simple experiment where they injected female prairie voles with oxytocin or saline and placed them in the cage with a male for 6 hours. The females were not sexually receptive, so they did not mate with the male, but females who received oxytocin spent more time with their partner than with the novel male, indicating a pair bond. So this molecule stimulates social bonding in voles.

One of the more interesting things about the vole system is that there are many different kinds of voles that display different behaviors. As mentioned, prairie voles are highly social, but different species of voles, such as meadow voles, which look almost identical in their physical appearance to prairie voles, display very different behavioral traits. They are not monogamous at all, they're pretty much solitary and don't crave social contact like prairie voles. Prairie voles form pair bond bonds; meadow voles do not. By doing comparative studies in these species, we can figure out what differences in the brain might contribute to these differences in the ability to form relationships. This is when I got involved in the research.

I wondered what is different in the brain of a prairie vole versus a meadow vole that allows one to form a bond and the other not able to do so. My first hypothesis was that prairie voles have more oxytocin than meadow voles, but that is not the case: they have the same amount of oxytocin. But what is different in the brain is where the receptors that bind to oxytocin are: they are concentrated in areas of the prairie vole brain that are involved in reward, reinforcement, and addiction. In the nonmonogamous species they are not there. So the receptor expression patterns in these guys changed evolutionarily to give rise to different kinds of behavioral patterns. We know that these receptors are the ones responsible for the pair bond because if you take prairie voles and inject directly into these reward and reinforcement areas an antagonist that blocks those receptors, the animals will not form a pair bond. Through these kinds of studies we can elucidate the brain mechanisms of bonding.

In male prairie voles it is a slightly different story. Although oxytocin may play an important role in male bonding, another peptide that we know is important is vasopressin. In other species, vasopressin stimulates territorial behavior and aggression but in these prairie voles it is involved in stimulating the pair bond between the male and the female. If you look in the brain to ask what is different between the male prairie voles that can form the bonds and the male meadow voles that cannot, you see again that the difference is in the location of the receptors. Monogamous prairie voles have high densities of receptors in the ventral pallidum, which is a major output of the nucleus accumbens and is involved in reward and addiction, while nonmonogamous species do not. If we do site-specific infusions of vasopressin receptor antagonist to identify precisely which areas are responsible, we find that the ventral pallidum is critical for pair bond formation.

Now I will discuss how we envision that these neuropeptides stimulate bonding in these animals. First, what is the role of oxytocin and vasopressin in this process? We know that these peptides are involved in recognition of individuals, or the neural processing of social information. We know this because if you take mice that have gene deletion for oxytocin or vasopressin receptors and expose them to other mice repeatedly, they do not recognize the other mice as familiar. In contrast, wild-type mice show a decline in investigation after repeated exposures, indicating that they remember the mouse they were exposed to. If they don't have oxytocin or vasopressin receptors, they have social amnesia and never recognize that mouse as familiar. This and a series of other studies tell us that oxytocin and vasopressin are important in social information processing.

In our model of pair bonding in voles we know that oxytocin and vasopressin act in the nucleus accumbens and ventral pallidum, which are both involved in reward, reinforcement, and addiction. The coactivation of the social information processing and reward systems produces a neural association, resulting in a conditioned partner preference: they form an association between the social cues of their partner and the rewarding nature of the social interaction and mating, and therefore they prefer to spend time with that partner. These kinds of studies suggest that social bonding is very much like addiction. In fact, we find that if they are separated from their partner, they show behavioral signs that are very similar to withdrawal.

We can transform the social behavior of one species into that of another through gene transfer technology. For example, we took meadow voles that have lower levels of expression of vasopressin receptor and, using a viral vector, injected the gene from the prairie vole into the ventral pallidum of the male meadow vole to make it have a vasopressin receptor pattern of expression similar to that of the monogamous prairie voles. We found that these animals were able to form social bonds in this species that naturally would never be able to form a pair bond.

Now I want to raise a very important question. Do the processes that we have discovered in voles related to oxytocin and vasopressin and social relationships have anything to do with human behavior? It very well could be that these little rodents have evolved completely different mechanisms and that what we're learning from them has nothing to do with humans. I will discuss several examples where there do seem to be some really remarkable parallels between pair bonding in voles and human behavior. 
Investigators have begun to explore the role of oxytocin in human behavior by giving intranasal oxytocin. If you sniff some of it, apparently it gets in the brain and then you can do all kinds of behavioral studies to see what it is doing. The first example is a study that came out in 2005 and showed that in persons who sniffed oxytocin, it increased trust (Kosfeld et al. 2005). This was an economics game where the subjects were told they had some money and could choose to give some portion of it to another person. If the recipient decided to give that money back to the subject, they would get three times that amount. The conclusions were that if one is given oxytocin, they are more likely to trust another person. Companies have taken advantage of this and one now has a product called Liquid Trust-they say if you spray it on you before an important business deal you will be trusted. Now they even have an improved product, Liquid Trust Enhanced, which has been specifically designed to "give a boost to the dating and relationship area of your life. This upgraded formula still contains the same great oxytocin, but now includes powerful pheromones." It is unlikely that these products actually work beyond giving the wearer more confidence.

There have been probably 25 or 30 studies looking at the effects of intranasal oxytocin on human behavior; I'll discuss a few of them. Not only does oxytocin enhance trust, it enhances the amount of time that individuals spend looking into the eye regions of other people-eye-to-eye contact. Other studies show that it increases "mind reading," the ability to infer the emotions of others using only subtle facial cues-for example, looking in the eye regions of someone and inferring whether that person is happy or sad. So it's reading social signals, similar to the mice study that I mentioned earlier.

A more recent study shows that oxytocin increases amygdala-dependent socially reinforced learning (Hurlemann et al. 2010). In this study subjects were given a task and if they made the wrong choice they got a frowning human face on the computer screen, and if they made a right choice they got a smiling face. If they took oxytocin, they learned this task much faster than if they didn't take it. That impact is specifically relevant to the social cues-if they're rewarded by a nonsocial signal such as a green or red light, it has absolutely no impact. This study suggests that oxytocin enhances socially reinforced learning. I believe that in humans oxytocin has the same effect as we find in animals: it enhances the saliency of social stimuli.

So now I think you can understand why we began to think about this in terms of autism, which I never thought about for the first 10 years of this research. Maybe [these findings] can help us develop treatment for social deficits.

I study complex social behavior such as pair bonding, but you can think about this bonding as a kind of social learning that involves different cognitive processes. For example, social reward and reinforcement, social information processing, and synaptic plasticity are all involved in social learning. Because of the work we've done in animals, we know some of the molecules that are involved in these processes, like oxytocin, which is involved in social information processing; dopamine, which is involved in reward and reinforcement; and glutamate and dopamine, which are involved in synaptic plasticity and learning. The idea is that if you could use drugs activating these systems to enhance any one of those cognitive processes, you might be able to enhance social learning, which might be incredibly useful in [treating] autism.

Several studies over the last few years have taken the lead from research done in animals, and in voles in particular, asking whether oxytocin is really useful in treating autism. Studies have suggested that giving intranasal oxytocin to individuals with autism enhances some aspect of their social cognitive function (Andari et al. 2010). So there seems to be a lot of promise in this line of research, but there are also some problems because oxytocin does not cross the blood-brain barrier efficiently. What's needed is a strategy to stimulate the oxytocin system more strongly, and that's one of the things we're doing at Yerkes. We're looking at oxytocin neurons in the brain, identifying receptors on those neurons, and identifying the receptors that have drugs we could give to individuals to stimulate those neurons to release the endogenous oxytocin in the brain. We have indentified one such receptor, the MC4R-it's not important what it is for this purpose, what is important is that there is a drug that has been in clinical trials for other purposes called Melanotan II that binds to that receptor, and this receptor has been shown to stimulate oxytocin release. We now know that if we give that drug to a female prairie vole before housing her with a male, she bonds even more strongly than if we gave her oxytocin. So here's a drug that we can give to drive the oxytocin system in the brain [and] stimulate the formation of social relationships.

I think this finding could be really critical because right now there are no drugs for autism that specifically target the social deficits. The best treatment for the social deficits are behavioral therapies that use socially reinforced learning techniques to teach the children social rules, how to read body language and social cues. It is possible that drugs that enhance the saliency of social stimuli-for example, oxytocin or drugs that stimulate oxytocin release-may enhance the efficacy of these behavioral therapies. Indeed, I believe that pair bonding in voles might actually be a very useful behavioral paradigm to test for drugs that enhance social learning and that, if given to autistic individuals just before social learning sessions or behavioral therapies, may enhance their ability to learn during that process and synergize with the behavioral therapy.

To conclude, from my research I have learned that animal models can provide insights into the mechanisms that regulate very complex behaviors in humans. You might think that rodents may not be able to tell [us much] about things like social bonding, but apparently they can. There are many parallels between the effects of oxytocin and social cognition between rodents and humans. These parallels may allow us to use animal models to develop novel treatment strategies for psychiatric disorders and drugs that can enhance social learning. 


\section{References}

Andari E, Duhamel JR, Zalla T, Herbrecht E, Leboyer M, Sirigu A. 2010. Promoting social behavior with oxytocin in high-functioning autism spectrum disorders. Proc Natl Acad Sci U S A 107:4389-4394.

Burbach P, Young LJ, Russell J. 2006. Oxytocin: Synthesis, secretion and reproductive functions. In: Neill JD, ed. Knobil and Neill's Physiology of Reproduction. Elsevier. p 3055-3127.

Hurlemann R, Patin A, Onur OA, Cohen MX, Baumgartner T, Metzler S, Dziobek I, Gallinat J, Wagner M, Maier W, Kendrick KM. 2010. Oxytocin enhances amygdala-dependent, socially reinforced learning and emotional empathy in humans. J Neurosci 30:4999-5007.

Kosfeld M, Heinrichs M, Zak PJ, Fischbacher U, Fehr E. 2005. Oxytocin increases trust in humans. Nature 435:673-676.

Ross HE, LJ Young. 2009. Oxytocin and the neural mechanisms regulating social cognition and affiliative behavior. Front Neuroendocrinol 30:534547.

Williams JR, Insel TR, Harbaugh CR, Carter CS. 1994. Oxytocin administered centrally facilitates formation of a partner preference in prairie voles (Microtus ochrogaster). J Neuroendocrin 6:247-250.

\section{Insight into Brain Development from Animal Vocalization Studies}

\section{Erich Jarvis}

'd like to thank the organizers for inviting me, particularly since I have had many discussions with my IACUC and OLAW representatives at Duke about how birds are not mice-different animal models require different living housing conditions and so forth. It's been a challenge for me to work not only with birds but, as you'll see in my presentation today, with many different species, taking a comparative approach of what traits a particular species has that humans or nonhuman primates or others don't have.

My talk today is a basic science presentation about insights into brain development from animal vocalization studies...[and particularly] insights into complex behavioral traits from these studies.

Overall, my lab is interested in understanding the mechanisms or the neurobiology of vocal communications in animal models to study how the brain generates, perceives, and learns complex behaviors. Our specific quest is to determine the molecular mechanisms that construct, modify, and maintain neural circuits for vocal learning, which is a critical behavioral substrate for spoken language. Language to many of us is considered the epitome of a complex behavioral trait. So we've taken on what I consider a challenging question but a necessary and possibly doable one using animal models.

I've also been asked to answer two broader questions: Why is it necessary to use animal models to answer these questions? I'll come back to that at the end but I'd like you to think about it as I go through my presentation and what implications this work might have for human health. I'll also add not only human health but also understanding of basic human biology, particularly for language.

What about the vocal learning trait? It is quite rare. Figure 1 shows a mammalian family tree on the left and an avian family tree on the right. I've highlighted with arrows the species that are known to be vocal learners, that have the ability to imitate sounds. Not everybody can imitate sounds quite as remarkably as humans do, but there are species that seem to have this trait and the vast majority that do not. Among primates, only humans have this ability-not nonhuman primates such as chimps, macaques, or squirrel monkeys. Bats also have this ability as well as whales and dolphins. More recently, it has been shown in elephants imitating truck sounds along the road. I recently saw a video of an Asian elephant putting its trunk in its mouth and moving its lips and producing Korean speech like its owner; I couldn't understand what it was saying but Korean speakers could.

Likewise there are very few bird groups-hummingbirds, parrots, and songbirds are the three that are known to be able to imitate. Many of you know that the word "parrot" has become synonymous with the trait of vocal mimicry. What's interesting about all four, five, or six of these groups is that they do not have close relatives that can imitate sounds. For instance, songbirds have a close relative, the suboscine songbirds, that are like the chimps of the songbird world: they're very closely related, belong to the same order, but don't imitate. Yet parrots and hummingbirds, which are far distant from them, do imitate. The phylogenetic distance between a hummingbird and songbird is about similar to that between a human and a dolphin.

When we talk about imitation many people think these are just animals mimicking, purely by rote, and don't understand what they're mimicking. Irene Pepperberg's work has demonstrated that parrots can understand what they are imitating even across species. She taught her famous parrot Alex how to count. [video: Look on the tray, how many total? Parrot: One.] You heard him say 1. The reason I show this video is not because 1 is a simple count-he could count up to 6 before he died-but because there are multiple things happening here. There is reciprocal communication between a human and a nonhuman animal. The nonhuman animal understands what the human is asking and can offer a vocal response, in human speech, with the answer to that question. Alex could get up to about three sentence words before he 
Figure 1 Convergent behavior: vocal learning - substrate for speech

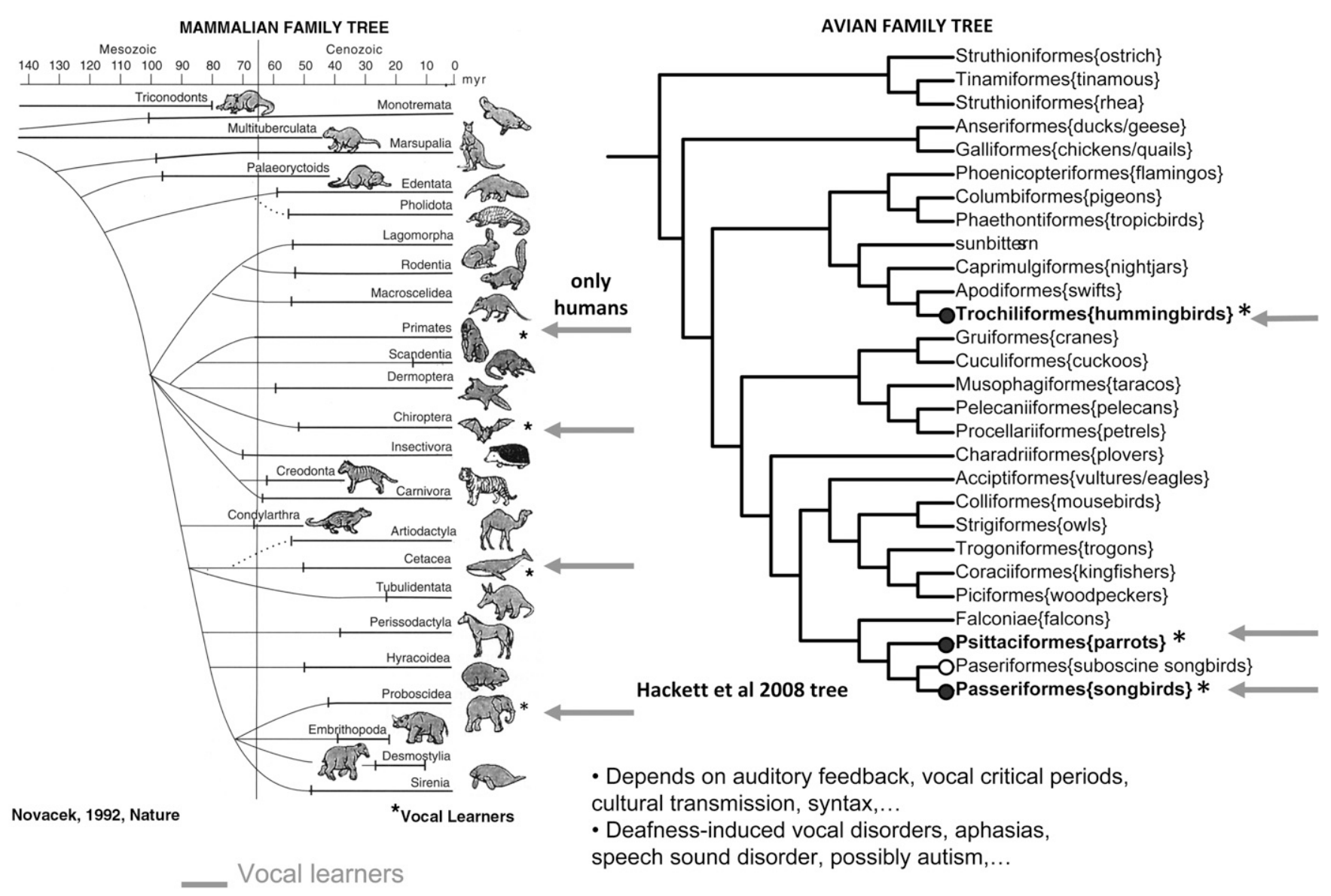

died. Not only could he count, but he's adding. The reason I like to show this video is the way he identifies one pea under that cup, he adds one plus zero, and he has a concept of zero, which was considered unique to humans before this study.

I don't think only vocal learners demonstrate these complex traits or cognitive abilities but they have an ability to express it for those who can imitate human speech. What is special about the behaviors of vocal learning species that nonvocal learners don't have? Table 1 lists some of them.

I then asked whether we could use song-learning birds as an animal model to study these traits for neurobiology. For behavior, we humans and song-learning birds have critical periods of vocal imitation: we learn best in early life and after that undergo crystallization in songbirds or puberty in humans, after which it becomes hard for us to learn another language or pick up new words, repertories. For some songbirds, they just don't learn at all past that stage in life. Nonvocal learning animals don't have that property.

Also in the vocal learners, there's auditory-guided vocal modification. That is, you need audition in order to learn and develop the sounds. It's not just that you hear it once and can then repeat it. Over time you need to practice. After you become an adult in both sets of species what can happen that doesn't happen in nonvocal learning species, including nonhuman primates, is that when we become deaf without speech therapy, our speech deteriorates, it becomes muddled. The same thing happens to song in these song-learning birds.

There are also syntactic rules and structure, learning of both, although we don't know if these have meaning in songbirds. There's recursion to embed ideas within an idea, or syllable structures within another syllable structure, and come back to the original structure. Syntax with meaning doesn't seem to exist in the song-learning birds (although it hasn't been tested that well), or grammar-that is, meaning to the actual order of the sounds. None of these traits can be found in species that can imitate sounds. There seems to be a package of abilities.

Now I'll quickly run through what we consider vocal learning systems in birds, what we've learned about them to inform brain systems in humans, and some new work we're doing in mice to ask where they fall. It turns out that mice haven't been studied for this trait even though there have been assumptions to the contrary.

What we've learned is that when songbirds produce their learned vocalization, that act of singing causes a robust increase of particular activity-dependent genes in the brain, [evidenced by].... a messenger RNA (mRNA) signal for an immediate early gene we call EGR1 or Ziff268.... Figure 2 shows a section of the brain with the cerebrum. When birds 
Table 1 Vocal learning: A critical behavior for spoken language

Critical periods for vocal imitation

Auditory-guided vocal modifications

Deafness-induced vocal deterioration - requirement for auditory feedback

Syntactic structure, rules, and learning

Recursion

Syntax with semantic meaning

Grammar

$\begin{array}{ll}\text { Yes } & \text { Yes } \\ \text { Yes } & \text { Yes } \\ \text { Yes } & \text { Yes } \\ \text { Yes } & \text { Yes } \\ \sim \text { Yes } & \text { Yes } \\ \text { No } & \text { Yes } \\ \text { No } & \text { Yes }\end{array}$

sing, this gene activation occurs in a linear fashion: the more the animal sings, the more induction we get in specific brain areas, like area $\mathrm{X}$-it is quite robust in the 30-minute window, where there is high mRNA accumulation such that there's a onefold increase for every song the bird sings.

We've demonstrated that it is a motor-driven gene expression response as a result of producing the learned vocalizations. When an animal is hearing and singing, compared to silent controls, you see the activation I just showed you and there's also activation in its auditory pathway brain areas. We used the molecular mapping approach to identify the auditory areas, which are analogous to the auditory forebrain in mammals. When the bird is hearing playbacks of its own song, you don't see activation in these song nuclei, but you do see activation in the auditory areas. When the animal is deaf and sings, there's still activation in the song nuclei but at lower levels than those in the hearing animals, not because of this particular gene but because deaf animals tend to sing less. (Other genes show association with deafness-induced vocal deterioration; I don't have time to show those.) In deafened animals, the hearing-induced gene activation in the auditory pathway is completely eliminated.

We asked, Is this phenomenon we observed in songbirds present in other vocal learning species that are distantly related in their phylogenetic relationships with songbirds? The

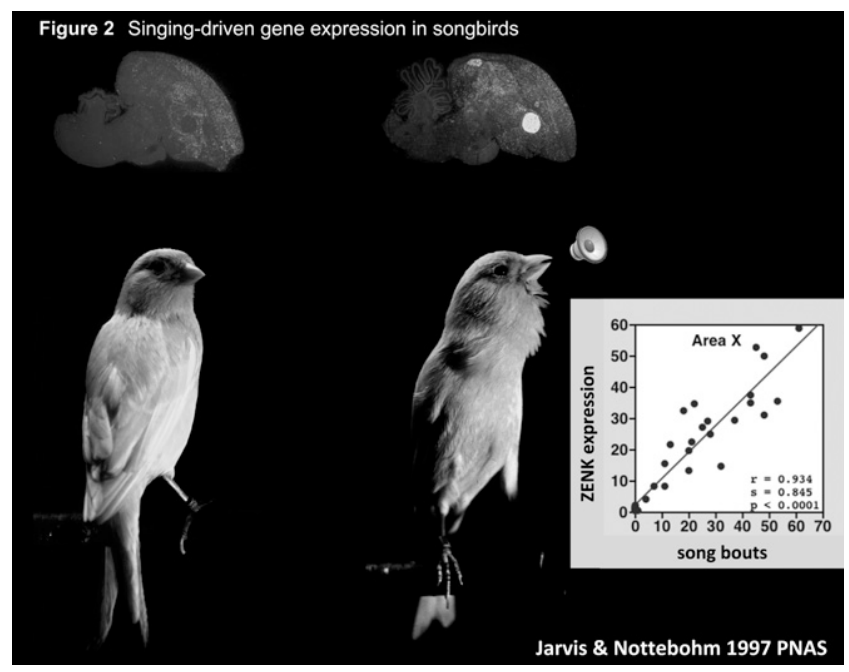

answer is yes. We applied the same approach to parrots and wild hummingbirds singing out in the wild and then looked at gene expression in their brains. Both the parrots and hummingbirds have hearing-induced activated areas of the forebrain and in the back of the brain, as in the songbirds. The forebrain activated areas are in similar locations although with different shapes. In the striatum and the cortical areas and the anterior part of the forebrain in hummingbirds and parrots, there are structures that look similar to the HVC nucleus, which was responsible for learned vocalizations.

When we put all this together we came up with a model as follows. Whether you follow one family tree of birds or another more recent view, what seems to have happened over the past 65 million years is that, three independent times, nature has evolved these vocal learning systems (colorcoded in red and yellow in Figure 3) that are involved in the production and the acquisition of learned or imitated songs. They consist of seven brain structures each, three of them (in red) are involved in the song learning process. The other four (in yellow) are involved in the actual production of the songs. So there could be three independent gains or maybe an independent gain back here and then a loss in the suboscines. That would be like the common ancestor of humans and chimps evolving speech and then humans maintaining the trait and chimpanzees losing the ability. All these hypotheses are quite remarkable.

All the species - including chickens or pigeons, which are nonimitators-have these auditory pathways that are involved in auditory learning, so we believe the song-learning birds inherited those structures from a common ancestor. All of them have brainstem areas that are involved in the production of innate sounds. What seems to have happened in the vocal learning species is this new forebrain system has taken over control of the more ancient system involved in the production of innate sounds. When we first published this, I didn't have a reason to say why it was happening - although I got communications from religious groups by email, letters, phone calls, and so forth saying that this result helps prove the existence of God because it's intelligent design, how else could you explain it? These pictures have ended up in religious texts for that reason.

If birds can come up with a similar solution in over 65 million years, at three independent times, what about 


\section{Figure 3 Convergent vocal learning brain pathways in birds and humans}

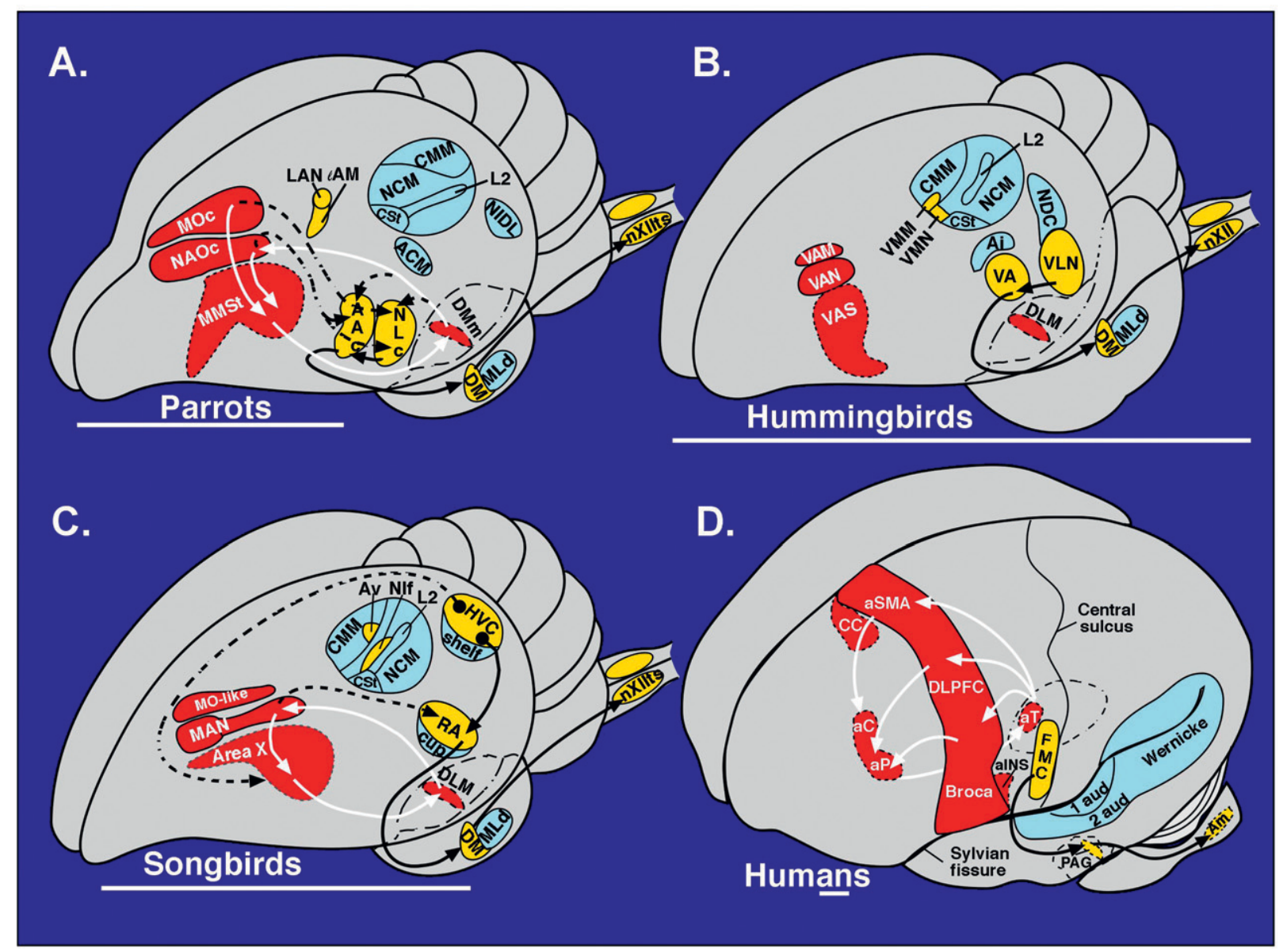

Anterior song/speech learning pathway found only in vocal learners

\section{Poster song/speech production pathway found only in vocal learners}

\section{Auditory pathway found in all species}

humans? Are humans going to somehow be magically different? Figure 1 highlighted the vocal learners on the family tree of mammals, suggesting that lions, tigers, and bears don't have vocal learning. It's been argued that vocal learners evolved it independently.

What about the brains of vocal learners? Figure 4 shows a songbird brain to scale with a human brain. You can fit roughly 3,000 of these into the human brain, so the first lesson is that brain size doesn't matter for this complex behavioral trait. Second lesson is that brain folding doesn't matter. Humans have a highly folded cortex whereas songbirds have a smooth telencephalon.

Over the past few years, a consortium of scientists that I organized has mapped out the homologies between the avian and mammalian brains. Songbirds have a large cortical territory relative to the rest of the brain. Unlike mammals, it is not layered; it's not nuclear in organization compared to the human cortex. Most of the song-learning nuclei are in the cortical-like territory, some are in the basal ganglia, which we call the striatum, or in the thalamus.
(Jarvis et al. Nature 2000; Jarvis 2004 Ann NY Acad Sci)

I asked, Is there anything in the songbird brain such that, when damaged through lesions, [it yields] results in behavioral deficits [comparable to those] in humans, such as aphasia patients, that you don't find in nonhuman primates or other mammals that are nonimitators or that you don't find in chickens or pigeons? The remarkable answer is yes. When these structures in the anterior part of the forebrain, song nuclei, are damaged in songbirds, the animals can still sing but they can't learn new songs. They also have deficits in sequencing their song. That's similar to what we find when there is damage to the interior parts of the [human] cortex starting in the lateral hemisphere called Broca's area going up to dorsolateral prefrontal cortices. They lead to certain types of aphasias, called Broca's aphasia, [which involves] problems imitating new speech and sequencing words into sentences and phonemes into words.

The same thing happens in the striatum and in the anterior thalamus, although not talked about a lot, because neuroscientists tend to think that the cortex is the god of everything. It's not. In nonhuman primates, areas where you 


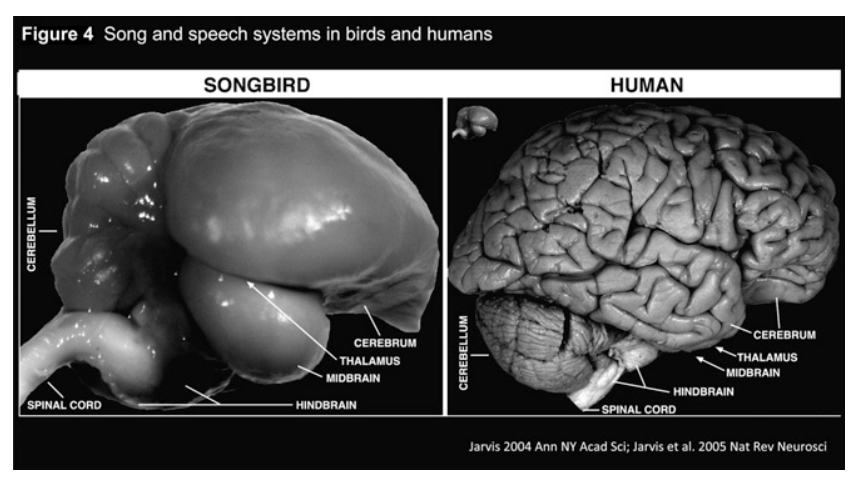

would expect to find speech or phasic damaged areas are connected in cortical-basal ganglia thalamic loops in the forebrain, and the loops are parallel in their connectivity to such loops in the song-learning birds. If you damage these areas in a nonhuman primate, or adjacent areas in a nonsongbird such as a chicken, nothing happens to the vocalizations. So here's a convergent trait for vocal learning, separated by 300 million years of evolution. Likewise, in nonhuman primates, these areas project to the motor cortex, particularly face motor cortex, which we know when damaged in humans leads to muteness for speech and in songbirds muteness for song. When the same experiments are done in nonhuman primates or in chickens, nothing happens to the vocalizations. You'd have to damage the midbrain areas in order to have effects on innate vocalizations in the other species.

I'm going to [discuss the] frontal section of the hemisphere. For the sake of time I'm not going to go into detail but there's a whole strip of cortex in humans that leads to certain types of aphasic deficits including Broca's area or poor emotional tone in speech in the cingulate area. On the left side you get verbal aphasias, on the right side you get what's called verbal amusias, difficulty singing. There's a dominance here-left dominance for speech in humans, and a left-right dominance for song in song-learning birds.

What I am arguing is that we humans have inherited an auditory pathway - I haven't gotten to the details of thisthat shares a common ancestry with the auditory systems in song-learning birds. I shouldn't say "inherited" but that we have acquired or evolved an anterior vocal pathway involving the acquisition and the production of learned speech that parallels what we see in these song-learning birds, but I believe it came about independently (not by intelligent design, though). How may that have happened? I have an answer more recently from some other studies, again in animals, where we found that not only when a bird sings is there activation in seven brain structures in the forebrain (six of them shown in Figure 5), but when an animal is moving its limbs in vocal learning species, you get this gene activation - think of this like an MRI-type of signal-in seven brain regions surrounding all the song nuclei, not elsewhere in the brain. The same effect is in parrots-hearing-induced gene activation, hearing- and song-induced, and induced by movement of the limbs. We found the same thing in hummingbirds. We found movement-induced gene expression in seven brain regions in quails but without the presence of song nuclei embedded in those areas.

What do I think happened? We have a hypothesis that for most vertebrates, whether humans or song-learning birds, ancestors of many that are living today have brainstem pathways involved in the production of innate sounds. They also have forebrain pathways consisting of an anterior forebrain loop and descending projections to control motor neurons to control motor learning. I argue that this pathway in the embryo develops in parallel in multiple forms, multiple times, to control different muscle groups. What has happened in humans and in song-learning birds is that, I argue, this motor learning pathway has duplicated itself and then forms as a new synaptic connection through mutations and acts on guidance molecules to synapse to motor neurons that control the innate vocalizations and merge those two pathways together to get an emergent vocal learning pathway that looks similar in distantly related birds and in humans. So there is a convergent mechanism to explain why the song-learning birds share similarities, not with complete overlap, but with each other and with humans. To put this theory in words: Brain areas that control vocal learning evolved out of preexisting systems that control movement and possibly motor learning. This can be applied to complex behavioral trait evolution in general, not just to speech. Again, this is a theory that we're now testing.

What about the genes that subserve those complex traits? Well, there's an interesting case of foxp2, which is considered a language-associated gene although in itself it is not a language gene-we demonstrated that it exists in crocodiles, [and] it was originally discovered in Drosophila. But humans have a specific mutation associated with speech evolution. Another specific mutation leads to speech apraxia-when one copy of in the genome is mutated, affected family members have difficulty acquiring speech.

We found, with my colleague Constance Scharff, that foxp2-like in the human brain is expressed in the striatum and that its expression goes up in the song-learning nucleus area $\mathrm{X}$ when juvenile birds are learning to imitate songs from adult tutors, during the developmental critical period. When they become adults, the foxp 2 gene is downregulated in area $\mathrm{X}$ after the bird goes through its crystallized pubertylike phase. Constance went on to demonstrate what happens if foxp 2 with RNAi was knocked down and the animal tutored (versus an untutored control). [Let's listen to] a sonogram of time and frequency of the sound. That's the father tutor [bird vocalizations]. This is the control animal [bird vocalizations] - the animal imitated, not perfectly but quite well. Now play the other tutor [bird vocalizations], and now play the foxp 2 knockdown [bird vocalizations]. This is what happens to humans - the human family members mix up the order of phonemes, slur the words, and so forth. These animals can't quite imitate, the sequence of vocalizations is out of order (Figure 6). So I don't think foxp2 was somehow inherited for the ability of vocal learning in humans and song-learning birds. I think once vocal learning evolved, this same gene got usurped for a similar function, to control 


\section{Figure 5 Song and movement areas have parallel connectivity}
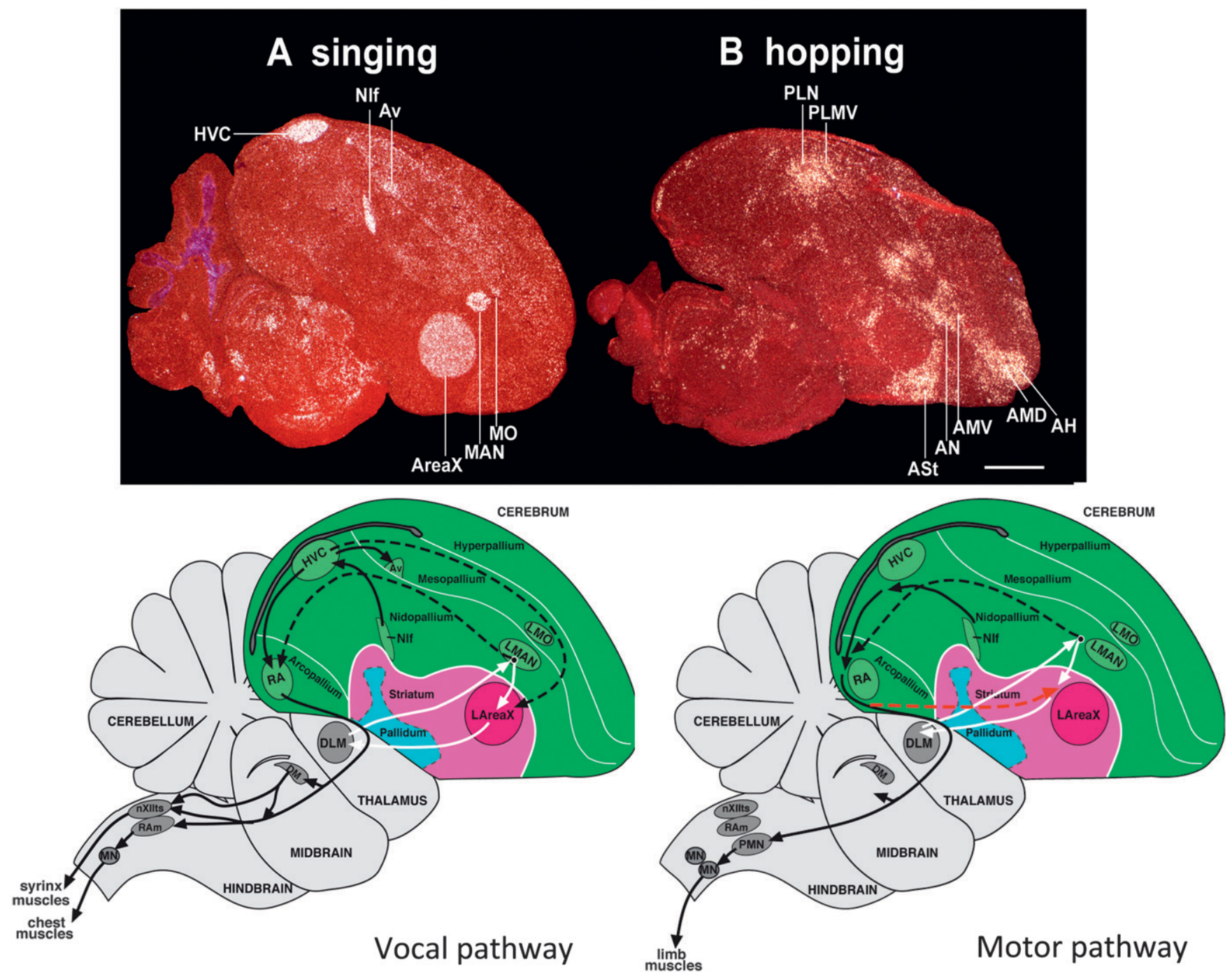

lyengar et al. 1999 J Neurosci; Bottjer et al. 2000 JCN; Feenders et al. 2008 PLoS ONE

motor-learned sequence behavior, in this case in the vocal systems.

I'm going to end with a story about mice because all this time we assumed that mice are not vocal learners until Tim Holy came out with a study. Mice make ultrasonic vocalizations...for courtship. Looked at in terms of their sequencing behavior, these vocalizations are sequenced similar to what songbirds do, so we wanted to use mice for controls for our vocal learning experiments, to try to induce a vocal learning system in them [in an] ambitious project we were working on. But then we asked, Do we have anything that resembles these vocal learners, like cortical striatal forebrain pathways or direct projection from the brainstem? From the forebrain to the brainstem, we tested six traits: three neurobiological traits and three behavioral traits that humans and song-learning birds have, that nonhuman primates and chickens don't have. For the sake of time, I will show you results from [just] some of these.
Songbirds and humans have forebrain areas activated during production of song or speech, as I showed before. You can show the physiological activity or MRI. We found that when mice "sing" they also have forebrain and striatal areas activated compared to silent controls when they sing (the areas are not activated when they hear a song but when they're deaf you can find activation). It's motor-driven, like we see in the song-learning birds, in M2 and M1 areas of the cortex. Humans and songbirds have these direct cortical projections to the brainstem that have fine voluntary control over the vocalizations; chickens and macaques do not have that, they only have these brainstem structures.

We asked, What about mice? We put a pseudo rabies virus in mice laryngeal muscle, backfilled the motor neurons in the brainstem, went up to secondary neurons to what we call the central pattern generators in the midbrain. These are all known to be present in all vertebrates, for 
Figure 6 RNAi knockdown of FoxP2 in songbirds
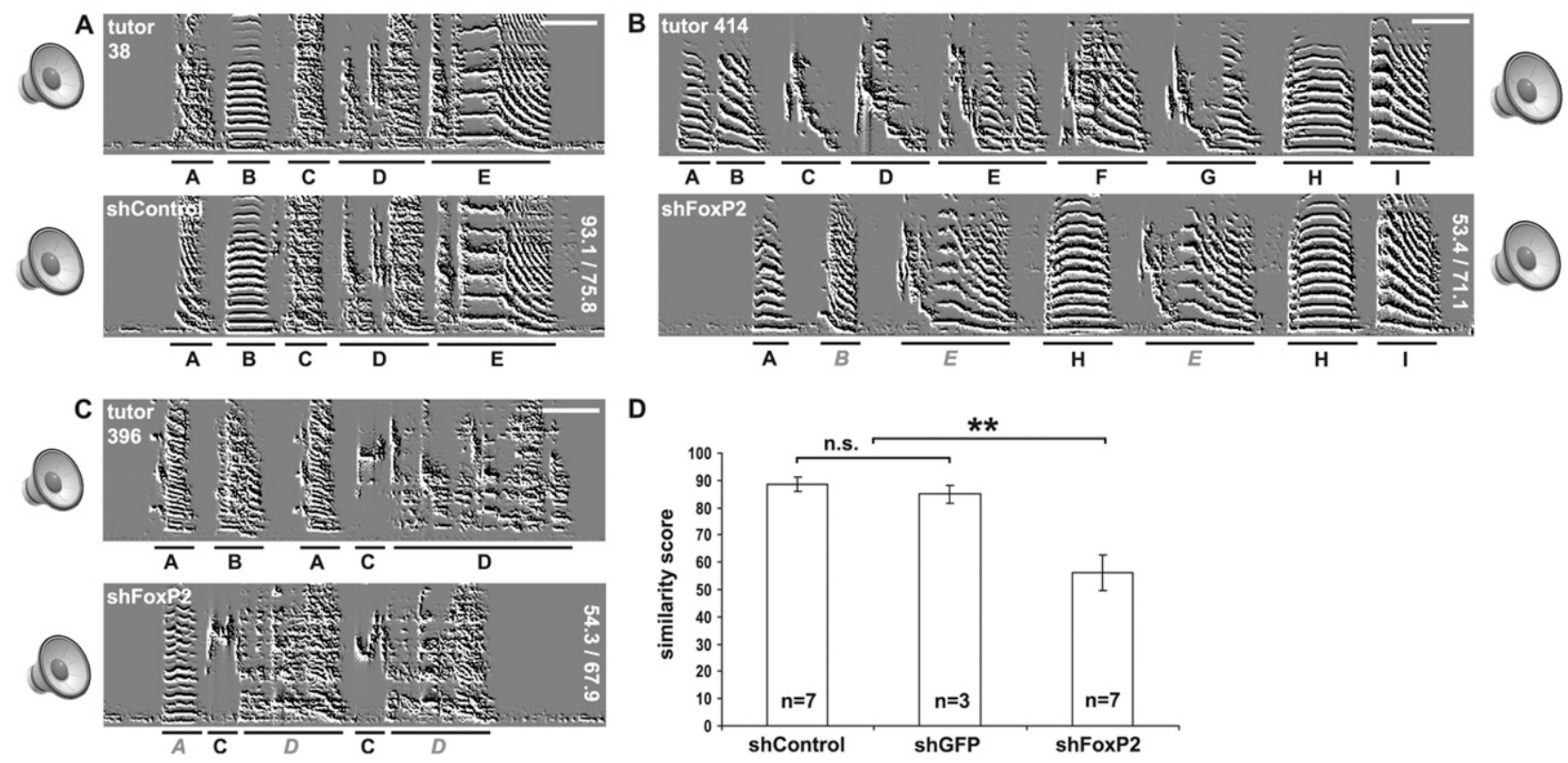

Haesler et al. 2007 PLoS Biology

control of production of innate vocalizations. But we found that mice also have a region of the primary motor cortex where we saw the singing-induced activation of neurons that make these direct projections to the brainstem motor neurons. We verified this projection by placing tracers in there and showing that axons from those areas of the cortex synapse directly onto the motor neurons that control vocalizations in mice. This connection has been said for the last 50 years to be the one defining feature that leads to the evolution of speech in humans and song learning in birds. Mice also, like humans but unlike nonhuman primates, show deafness-induced vocal deterioration....

We have also been able to demonstrate after many tries of many situations that we can get mice to shift the pitch of their vocalizations - one strain [will] match the pitch of another strain as a result of social housing together. [We did this by pairing] C57 male mice with a BXD substrain that sings at a different pitch. (If the male was not paired with a female, the mice would not shift in their vocalizations.) This animal, hearing this one singing, pitched his vocalizations down, so we got some form of imitation here in the mice as well. These are traits that are thought to be unique to humans, and are still published as such in textbooks today. Therefore people have argued you can't use animal models for them, but we're demonstrating that they exist in these mice.

I'm going to end with some questions. Why is it necessary to use animal models to answer these questions? I hope it's become pretty obvious that it's impossible to answer them without using animal models. How could I have demonstrated everything I just talked about without studying the animal? The other question is, What implications might this work have for human health? We can study speech disorders such as deafness-induced vocal deterioration and stuttering, foxp2, dependence on language as well as social communication functions with parallels to human speech. We need to be aware, though, that a mouse is not a songbird, a songbird is not a human, and a macaque is not a human either. There's a continuum, and we need to study the basic biology to understand that continuum so that, when we study the disorders of this basic biology, we can know the limitations for each species. 


\section{Insights into the Brain and Aging: Discussion}

Dr. Zola: We have some time for discussion or questions. I'm going to ask something from Erich and Larry first. Given your last description of work with mice, Erich, maybe voles would be the species you want to do that with because they already have this relationship and you might be better able to see aspects of imitation in voles than in a species that is not so genetically attuned to that.

Dr. Young: I wonder if the males serenade their female partners at some point?

Dr. Jarvis: [Steven] Pinker and others have a hypothesis that what selected for speech and language was actually mating, and the fact that we can get these mice to modify their vocalizations whenever there's a mating situation might speak to that.

Questioner: I'm a laboratory animal veterinarian and I'm on the faculty at the University of Pennsylvania. I really appreciate the talks-for someone like myself who's really passionate about working with animal models, this is an incredible example. It is impossible to do this work without the responsible use of animals. There's a subject that's been threaded through the last 2 days...: How do we push the scientist along in an age of increasing regulatory layers with the new Guide and expectations from all of the different agencies that have talked today? As a person that sits on our IACUC, I feel that we're getting a lot of pushback from the scientists. They say, "It didn't used to be this way. Three years ago when you reviewed my protocol you didn't ask for these same things. You're putting me out of business." I'm wondering, from the scientific perspective, from these esteemed institutions where you work, what can we do for everyone else to let them know the importance of the work but also the fact that it has to be done hand in hand with this increasing regulatory burden, as it's called? How can we bring the scientist more into the fold on this and not have such pushback, which I feel personally?

Dr. Young: I don't really have an answer to that question other than to say that I think there has to be a lot of communication, education of the PIs. I know that because I sat on an IACUC for 6 years and that gave me a real appreciation of why investigators get these rules that they have to follow. I understand why I have to jump through the hoops that I have to jump through. I think we have to educate the investigators as to why is it important to comply with these rules.

Dr. Jarvis: I have some beliefs about this. I think sometimes rules get set up, they can be 25 years old, for example, and they apply to certain situations, and sometimes rules are followed without actually explaining why. PIs need to be educated on why a rule needs to be followed, even though that person wasn't doing it 5 or 6 years ago. Then I think in most instances the scientists involved will understand because hopefully they're thinking logically and reasonably—that's their job. Also, regulation is mostly meant to protect the animals, [but] you have to be careful that it doesn't stop the creativity of science. As Einstein's famous saying goes, "If we knew what we were doing, we wouldn't call it science." Sometimes I feel that scientists are asked to predict exactly what's going to happen in their experiment-the exact number of animals and so forth, all the control groups-and if [I] could do that, I would be rich. So my answer is that there has to be some balance between flexibility and logical explanation.

Dr. Hopkins: I think you posed the central question really well. I think that is the issue and I think the tension is not going to go away easily. I think some tension depends on the individuals you're dealing with. It's a people-people issue and if one side understands the reasons for it, I think that helps a lot. On the other side, sometimes you come up to somebody who says this is the rule but actually, if you sit down and really look at the procedure, you can devise a procedure that's easy enough so people will do it without being upset by it. It's a process and if the process goes well, you get the end results you're looking for. But I think that there is too much pressure on people trying to do science today for a lot of different reasons and so I think that we're not going to see this ease up any time soon, unfortunately.

Questioner: Is your training program for your PIs a computer-based training program or is it personalized with a live, experienced individual able to answer questions?

Questioner: We do training and I think that's part of the problem with this because if there are any noncompliances, we are asked as a committee to retrain. We do hands on, we have pushed more and more toward online training so that everyone can sit at their desk comfortably and go through it as time allows and answer emails and everything else they have to do during their day. I agree that that's not necessarily the best way to train but I think as we learned yesterday that's where the future's going. We're all moving into webinars and remote access learning. One of the things that worries me a little about the new Guide, where we're going to have some issues with the science, is one of the points that Kathryn Bayne made, that we're supposed to begin to question the science with the use of the animals a bit more. In my training, it's always been that we are just looking at animal welfare as an IACUC, we're not supposed to question the science. I completely agree with you about making sure that we provide an explanation, personally I think providing data, the more we can get into the literature about the benefits of enrichment or animal welfare benefits in a publication format back to the scientists that will be most helpful but it's not always there. A lot of this still is anecdotal and gut 
feeling and public response and pressure. So I think in training at the University of Pennsylvania we have about 1900plus active protocols with maybe 500 to 700 PIs. I don't know how we, as a committee of 20, can take on the task of one-on-one training, unfortunately.

Questioner: In my recent years as a consultant and also serving on a number of IACUCs I have noticed the power of personal interactions. I had experience in one IACUC that will remain unnamed but the practice at that IACUC was for members who were reviewing the protocols to communicate directly with the PI. [In contrast,] I've seen a trend where, at many institutions, the IACUC questions get put to a central location for the purposes of anonymity and the professional staff then poses the question to the PI and so it becomes a three-way communication, very impersonal, sometimes not exactly on target. I think the direct communication adds a tremendous amount of understanding and a little more trust. So I have become a strong advocate of direct communication between the reviewer and the scientist.

Dr. Jarvis: Yes, in my own experience that has worked better because then you understand the rationale for the thinking of the IACUC more directly, and the IACUC understands the rationale for the thinking of the scientist. That certainly has been my experience.

Questioner: One of my roles is executive chair of the IRB and I also serve on the board of directors of [a large] independent or commercial IRB. I've been doing that for 2 years and I've learned a lot about customer orientation. I instruct our IRB staff that the investigators are our customers [and that] we're there to help their research be conducted ethically, scientifically, and in full compliance with all applicable federal requirements. We find it very important to have personal communication with investigators as opposed to impersonal communication. If, for example, you table a protocol, rather than send a fivepage letter the chair of the IRB or the IACUC needs to communicate directly and personally with the investigator and explain the committee's concerns and take that opportunity to educate the PI. I have found over my career that investigators want someone to listen to them, to understand the pressures they're under, to help them, rather than have impersonal, bureaucratic, inhibitory committees reviewing protocols and not facilitating their research. So I think IACUCs and IRBs need to be much more customer oriented.

Dr. Young: I agree with that as well. One thing to keep in mind is that you're constantly getting new faculty, new PIs as postdocs transition to faculty positions, and we don't get trained about the role of the IACUC. We know something about it but we don't really understand why we have to do all the things we have to do. I really do think there should be some sort of training program for new faculty to let them know what's going on.

Questioner: I have a quick question for Dr. Young about the prairie voles: What is their divorce rate? [laughter]

Dr. Young: That is an interesting question. In the wild, if they lose a partner about $75 \%$ of them never take on a new partner, so it seems to be a pretty strong bond. And related to that question is that, in the laboratory, if we separate them from their partner they show depressive-like behavior-they develop a depression and it's very much like withdrawal from a drug. We think that that negative affect from being away from the partner is what maintains the relationship after all the dopamine is gone. You can think about it in human relationship as well, [that] withdrawal maintains the relationship.

Dr. Jarvis: Larry, I want to add a comment and ask a question as well. Most mammals are not monogamous, very few are. I'm not quite sure where humans fall but there's social monogamy and obligate monogamy, social monogamy meaning that you're together but do pair copulations. I know most birds are socially monogamous.... I think only $3 \%$ of mammals are monogamous, whereas $70 \%$ of birds are, and it' $d$ be interesting to do comparative work there. Are prairie voles obligate monogamous as well?

Dr. Young: No, they're socially monogamous, very much like humans, basically. If a male prairie vole is wandering around and a female who is in estrus comes by he might mate with that female but the important thing is that he comes back to his nest with his partner and family at night and maintains that relationship. That's what we're studying, that social bond, not monogamy per se.

Dr. Jarvis: Do you think that prairie voles have a stronger socially monogamous behavior than humans? I'm sure some people want you to study that.

Dr. Young: No, I don't think they do. One of the nice things about prairie voles as a model system is that you can study them in the laboratory but there are also wild populations so [researchers] are doing complementary kinds of experiments in the field. When you look at voles in the wild you see that only about $60 \%$ of the males form a bond with a female; $40 \%$ of them are wanderers, which adopt a different strategy and mate with other males' females. So there's quite a bit of diversity, just like in humans. I don't think that they're any more monogamous than people.

Questioner: With this all-star cast on the podium, I can't resist asking another question about science. Stuart talked about memory and Larry talked about social differences. My question is generated by an observation we've made studying bonobos and comparing them to chimpanzees. We suspect that many of the behavioral differences between these two species may be driven by developmental differences. One of the surprising findings we have is that bonobos have a very hard time remembering where food is hidden relative to chimpanzees. My question is, With the prairie voles have you studied memory at all and are any of the social behavioral differences you see an epigenetic phenomenon?

Dr. Young: We haven't really studied any other kinds of memory besides social memory in the voles. But in terms of epigenetics, we talked about enrichment earlier and how nature versus nurture influences behavior. We have some studies to look at social behavior of prairie voles in families where both the male and the female parent are present versus those raised only by the single female parent; we quantify the amount of licking and grooming and nurturing behavior 
that the pups get as they're growing up — and they get different amounts. Sometimes, if the female wants to get off the nest to get food, she'll actually grab the male and pull him to the nest and he'll stay there while she gets the food. We found that the pups raised by the single female parent were less likely to show alloparental behavior to others and also took longer to form pair bonds. We looked in the brain and saw differences in expression patterns for some of the variables that contribute to this behavior. I think that's a good example of how variation in enrichment can cause a variable: the presence of a single parent or both parents produces a different sort of brain organization that gives rise to differences in behavior, so it should be considered a variable in the experiments. But it's also interesting in its own right.

Dr. Zola: Just to add another dimension to that. We know there's more than one kind of memory. The memory we've talked about was declarative memory that is hippocampally based, but there are many other structures and regions of the brain that we now understand are important for other aspects of memory. If you lose your entire hippocampus, there are some kinds of memory tasks that you can still do quite normally even though you won't remember the episodes of learning or you won't consciously be able to recollect any of the training going into it. You can imagine, then, that environmental evolutionary constraints might lead some organisms to have certain special kinds of memory skills and others to have other kinds of memory skills. We're just beginning to tap into that possibility.

Let me just add one other thing, related to a point that Nancy made, in terms of what we're facing as we get older. As we age, what typically happens is a gradual progressive diminution of our cognitive and other abilities. But what we are really looking for is a kind of step function, not a gradual curve. Some have described it as the step function of death. You want to be going along very well and then things just stop. You do not want to be subject to age-related life-changing conditions that progressively ruin your quality of life. The hope is that we can sustain our quality of life for a very long period of time and that the disintegration would be a very short part of the curve. 


\section{Standing Up for Science: Session Introduction}

\section{Jerry Collins}

any of us in this room believe strongly that the use of animals in biomedical research is essential for the improvement of human health. The Public Health Service Policy is what we are here to commemorate, but we also, in our celebration of the advances that have been made, need to recognize that there are some very significant challenges still facing us. We have two individuals who are going to share with us both their views on some of those challenges and some of the ways that we can perhaps work together to address them.

The first is Dr. David Jentsch. He received his bachelor's degree in behavioral biology from the Johns Hopkins University and his $\mathrm{PhD}$ in neurobiology from Yale University. After postdoctoral training at the University of Pittsburgh and then back at Yale, he moved to UCLA, where he is currently a professor in the Departments of Psychiatry and Psychology. He is also the Associate Director for Research for the Brain Research Institute. His research focuses on genetic and neurochemical mechanisms that influence cognition, impulse control, and decision making in laboratory animals.
In reaction to the escalating extremism among animal rights activists, which culminated in the firebombing of his car in 2009, Dr. Jentsch formed the group Pro-Test for Science. He and his colleagues play a prominent role in scientific advocacy by coordinating the response of the scientific community to attacks against researchers. Dr. Jentsch is also a member of the board of directors of the biomedical advocacy group Americans for Medical Progress.

Dr. Dario Ringach received his BS in computer engineering and MS in electrical engineering from Technion, the Israel Institute of Technology, where he did research in computer vision and image processing. He later shifted his attention to the biology of vision and received his $\mathrm{PhD}$ in neuroscience from New York University. He's been at UCLA since 1999 and is now a professor of neurobiology and psychology. His scientific interests center on cortical dynamics and computation. He also devotes an increasing amount of time to advocate for animal research and promote a public and civil dialogue on the use of animals in biomedical research.

\section{Ways Individual Scientists and Clinicians Can Support Animal Research}

\section{J. David Jentsch}

t's been inspirational for me in a number of ways to be here for the past 2 days: one is as a scientist and the other is as someone who has spent quite a lot of time over the last few years investing in the system for ensuring animal welfare by being a member of my campus IACUC. So it's been interesting to see these various parts of my life-the scientist part, the animal welfare part-played out in the same meeting, which is not very common.

I want to share some of my thoughts about the ways individual scientists and clinicians can support much of what we've talked about here today. I'll start by talking about why it's important and, indeed, necessary that they do this, and end with methods and ideas about how to perform this advocacy role.

There are some basic facts that must be underscored. These are facts that we can't avoid and we shouldn't avoid.
The first is that biomedical research is arguably the single most noble use of animals in our society because the use of the subject has the broadest benefit — not just for the human race but for animals as well. If you eat a steak, you benefit yourself. If you conduct research into the fundamental mechanisms of biology and health, you benefit everybody. On top of that is the second fact, which is that we have strong ethical principles. These ethical principles are represented in the Animal Welfare Act. They're represented in the relevant agencies' regulations that relate to animal welfare and the conduct of science. But these laws and regulations are not the source of our ethics. They reflect the ethics of those who participate in animal research. The Animal Welfare Act and PHS Policy stemmed from the ways that the vast majority of scientists conducted themselves in the laboratory, the sensibilities they already had about animal research. 
These facts have to be taken in light of another fact, which we have already discussed. Table 1, which exhibits the results of a 2009 Pew Research Center poll, demonstrates that there is a mixture of opinion in terms of the broader public - their personal feelings and comfort levels - with the use of animals in scientific research; some polls, including this one, show that as few as $52 \%$ of people appear to endorse animal research, suggesting that a large block is more critical [of it]. Many people would characterize these apparently critical opinions as opposition. I argue that this number reflects a moral ambivalence about the use of animals in research rather than opposition to it. Notably, this public moral ambivalence is enormously different from the acceptance of animal research by scientists, which exceeds $90 \%$ [and includes not just animal researchers but] physicists, chemists-scientists of the broader category who look at these facts and conclude that this is an acceptable and, indeed, welcome use of animals.

Table 2 is a 2010 Gallup poll. You'll see that the proportion of individuals critical of animal research varies with how the question is asked, which is not unusual with polls. But I thought it would be useful to present the entire Gallup poll to draw your attention to other items that were included in it. As you can see, about 59\% of individuals find the use of animals in research to be "morally acceptable," but compare that to other items [from the poll]. It's basically the same percentage of people who find it to be morally acceptable for gays and lesbians to be involved in stable romantic
Table 1

\begin{tabular}{|c|c|c|}
\hline \multicolumn{3}{|c|}{$\begin{array}{l}\text { Differences between Public and Scientists } \\
\text { Go Beyond Evolution }\end{array}$} \\
\hline $\begin{array}{l}\text { Think that humans, other living things } \\
\text { have evolwed due to natural processes }\end{array}$ & $\frac{\text { Public }}{\%}$ & 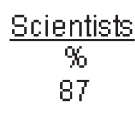 \\
\hline $\begin{array}{l}\text { Think that earth is getting wamer } \\
\text { because of human activity }\end{array}$ & 49 & 84 \\
\hline Favor use of animals in scientific research & 52 & 93 \\
\hline $\begin{array}{l}\text { Favor federal funding for embryonic } \\
\text { stem cell research }\end{array}$ & 58 & 93 \\
\hline Favor building more nuclear power plants & 51 & 70 \\
\hline $\begin{array}{l}\text { Say that all parents should be required } \\
\text { to waccinate their children }\end{array}$ & 69 & 82 \\
\hline
\end{tabular}

relationships. It's about the same percentage of individuals who find the use of embryonic stem cells to correct disease to be morally acceptable.

So the moral ambivalence is not unique to animal research. It's embedded in a broad range of public attitudes about controversial topics. The different items [shown in Table 2] have very little in common, superficially, with one exception. For each of the items where you see this moral ambivalence you also find a very concerted, persistent, wellfunded, loud set of "anti" voices expressing opposition. We've seen it in California, recently, in the case of Proposition 8.

\section{Table 2 Gallup poll results showing that four moral issues sharply divide Americans}

Perceived Moral Acceptability of Behaviors and Social Policies

Ranked by difference

$\begin{array}{lccc} & \begin{array}{c}\text { Morally } \\ \text { acceptable }\end{array} & \begin{array}{c}\text { Morally } \\ \text { wrong }\end{array} & \begin{array}{c}\text { Difference } \\ \text { pct. pts. }\end{array} \\ \text { Doctor assisted suicide } & 46 & 46 & 0 \\ \text { Gay or lesbian relations } & 52 & 43 & 9 \\ \text { Abortion } & 38 & 50 & 12 \\ \text { Having a baby outside of marriage } & 54 & 40 & 14 \\ \text { Sex between an unmarried man and woman } & 59 & 38 & 21 \\ \text { Buying and wearing clothing made of animal fur } & 60 & 35 & 25 \\ \text { Medical testing on animals } & 59 & 34 & 25 \\ \text { Gambling } & 61 & 34 & 27 \\ \text { Medical research using stem cells obtained from } & 59 & 32 & 27 \\ \text { human embryos } & 31 & 63 & 32 \\ \text { Cloning animals } & 65 & 26 & 39 \\ \text { The death penalty } & 69 & 23 & 46 \\ \text { Divorce } & 15 & 77 & 62 \\ \text { Suicide } & 9 & 88 & 79 \\ \text { Cloning humans } & 7 & 90 & 83 \\ \text { Polygamy, when one husband has more than one } & 6 & 92 & 86 \\ \text { wife at the same time } & & & \end{array}$

May 3-6, 2010

\section{GALLUP'}


We've seen it with respect to testing on animals. We've seen it with respect to the therapeutic use of human embryonic stem cells. And look at climate change. It's the same scenario. This moral ambivalence almost always correlates strongly with a conservative, well-funded, and frankly, in many cases, antiscientific/-science denialist rhetoric. Our opponents have strong messages. They're out there. They're well funded. They're concerted. They're well tuned. Yet, outside rooms like this, our messages are lacking, even though we believe strongly in the moral acceptability and importance of animal research. That message is not translated often, and not translated in a way that has an impact, nor does it reach the same number of ears that the "anti" voices do.

Why is this the case? Why are people who believe in the crucial value of biomedical research involving animals not out there giving the public our message? First, of course, is because we're busy, but so are most people, so that's not a very good reason. Second, we're not necessarily used to discussing the nuances of science with nonscientists. I feel a little ambivalent about that statement after hearing the presentations today, which I think were quite remarkable in their ability to convey important messages about biomedical research to a broader public. Third, and perhaps most importantly, we sometimes don't really believe that there's a problem, or, if there is one, that it applies to us. We don't see this moral ambivalence in our day-to-day lives. We don't see how it relates to the potential future of biomedical research, and maybe think that it applies only to the West Coast and not our own neighborhood.

But, quite acutely, there is another issue, and that is that scientists are afraid. They're afraid to communicate about what they're doing openly because the "anti" voices are so strong and because, unfortunately, in this case, they carry sticks. The sticks they carry come in many different forms. Sometimes the sticks are used to have an impact on the scientific research institutions where research is conducted. We've seen this in many different cases, ranging from criminal vandalism of the institutions to something that is more common today, which is the use of the almighty dollar to influence the scientific agenda of research institutions. This has happened recently.

On the enterprise level, legislative efforts to change, inhibit, or steer the course of science are often embedded in public relations efforts that provide less than complete information (and that's being generous) about the impact of this legislation on biomedical research.

Perhaps most distressing for scientists around the country is the ever increasing use of personal attacks, including the use of harassing electronic communications, protests at scientists' homes, and threats of other forms of violence.

Why do people do this? What has triggered this transition over time-from trying to influence the government, the local institution, to attacks on individuals? Mostly, it comes down to efficiency. Attacking people is cheap and it's effective. Trying to influence the government is difficult and slow. The movement is full of all sorts of people who try to use whatever method is at their disposal to achieve the desired end: no more biomedical research involving animals in the United States of America. Many activists opposed to research ask, "How do we accomplish that end?" Unfortunately, the movement is full of others whose position is, "Who cares? The end is what matters. We have a job to do and we'll get there any way we have to."

The point is that there is a tremendous personal cost of being a voice for biomedical research, and that's a challenge. It's difficult for people to understand; it's difficult for people to accept. That said, despite the personal costs that come from being a targeted individual, speaking about research is an obligation. It's an obligation for many reasons. First and foremost, absent all of these other issues, the public supports the work that we do through a very large financial investment (through the NIH and NSF and so forth) and has a bona fide right to understand what we do and why.

On a separate note, many of us have the word "professor" before our name. Professors are, by definition, educators. Teaching and facilitating learning is the first mission of most of the people who have this title-or at least it should be.

And a final important reason to join the debate over animal research is that our colleagues are under attack and they deserve our support, even if we feel that the threats themselves don't apply to us. It's important to remember that the opposition to animal research is essentially a moving target. There is a set of issues today (say, the use of nonhuman primates in biomedical research); when those issues are conquered, others will arise, and they will continue until all biomedical research using animals is stopped in this country. Those who oppose the use of animals will eventually come for every scientist who uses animals.

The question is, What can you do? When research is threatened or criticized, we have to work together as a community of scientists and academics to oppose those threats. To do so is to defend the cutting edge of science. Great science always provokes controversy and opposition because it's innovative, it's new, it's pushing the boundaries of what we know and our experience. It will always produce opposition, and it's our role as academics to oppose that.

Foremost in this task should be the leaders of our respected academic institutions; they must, without hesitation, support the research programs at their university and the investigators who undertake them. Accomplishing this will require you to work with your campus administration. You should go home today and talk with the members of your campus administration about what they're going to do to promote the research agenda of their university.

But don't just leave the task to them. Join them in advocating for (at the least) your own research because you know the science better than anybody else. You are the scientist; it's your work. Work with media offices and make sure they know of scientists who will openly discuss research, and use these methods to coordinate effective public outreach about the research that is being carried out in your university. Use the mechanisms the campus has to advocate for what's going on. Describe how you've adjusted and refined your research 
to attend to issues like animal welfare while accomplishing your important scientific goals. In my case, all my animals live socially in outdoor enclosures. That's not what antivivisectionists would tell you about what I do, but it's the truth. Use what avenues are available to you to describe your research. At UCLA, the campus is really deeply invested in promoting research. UCLA took out a full-page ad in the Los Angeles Times in 2009 to describe the biomedical research effort of the campus. Actions like this strongly support the endeavor to promote and advocate for researcher and researchers.

What other things can you do? You can take a visible role yourself. Speak openly about what you do when given the chance. Grad students are watching professors. They're learning how to talk to the public about research, and when they see you do it, they'll do it themselves. Never forget that reporters are, in fact, the method by which you communicate with the public and when they call, you should answer. If they have a question about something you're doing, that usually means the public has a question about something you're doing — and you should answer it. Collaborate with the media relations office in your university. Ask them for help and advice but don't shy away from speaking for yourself. For scientists, a first reaction to talking to a member of the lay public is sometimes "Why would I want to do that?" The answer is "because lots of people want to understand why we do what we do and what we're doing and want a direct line of sight into the research laboratory."

Some people don't feel comfortable talking directly to the media, but there are other options available. There are opportunities for controlled messages. One of my colleagues, Professor Edythe London, wrote a very powerful piece in the Los Angeles Times in 2007, "Why I Use Animals in My Research." Even years later people mention this piece and its impact on them. And of course there are yet other ways in this new generation to talk about what you do and to support your research efforts by contributing to blogs like Speaking of Research and others.

Basically, it's up to us. We're scientists and participants in science-veterinarians, veterinary technicians, laboratory animal researchers, scientific staff, research associates, and so forth. It's all up to us. This message needs to be propagated properly - it needs to be made by us. We're not going to be able to rely on anyone else to do it. It's got to be made by us and if it's not made by us, it's not going to [address] the contrary view. We have to be forceful, persistent, organized, thoughtful, and most importantly, we need to take home the messages we've heard these last 2 days and spread them as widely as possible. It's up to scientists and the people who work in the scientific enterprise to do this. If you're not $100 \%$ sure about what messages you want to give to the public about your research, you can embed it in the broader scientific context we've talked about today and the broader context of animal welfare we talked about yesterday. You can also use a number of resources that are available to help hone your ability to communicate your personal passion for science to the public; Americans for Medical Progress and Speaking of Research are two that I would like to highlight.

\section{The Need for Public Dialogue}

\section{Dario Ringach}

think what's missing is true bidirectional dialogue between those that support the use of animals in research and those that oppose it. I decided that it's worth spending a substantial amount of time trying to engage in such a dialogue - a productive dialogue — with those that are willing to listen to both sides and to understand why we do the research that we do and why it's important. Some may not be willing to participate in such a dialogue, that's fine. But I think those that are willing to participate should be invited. We heard, in the first session yesterday, complaints about those that oppose animal research not being invited to participate. Perhaps this celebration is not the best opportunity to do so, but I think that in the future we should foster circumstances where dialogue can happen.

What is dialogue? Why is it important to have a dialogue? Who should engage in dialogue and how can we encourage it?
Dialogue is the most difficult of the many outreach efforts that David [Jentsch] mentioned. It involves a difficult challenge, which is listening to the other side. Many of the things we do are not dialogue. We publish our work in scientific papers that the public can go and look at. In a way we can say our work is public: you can go and look it up, the information is there-and that's it. Providing information about our work and the regulations that are involved is valuable as it is important for the public to understand how the work is done, but it's not dialogue. We can write opinion pieces in newspapers about our opinions and feelings about animal research; still, it is not dialogue. We can have television programs or other events that explain the careful work with animals in laboratories, but it's still a one-way street.

There are many other ways in which you can try to engage with opponents of animal research. One can have blogs 
with comments that allow for some back and forth. Some public discussion can be conducted in panels, debates, point and counterpoint articles, interviews with journalists that will present both sides of the story. And, finally, one can have face-to-face contact.

Of all the different things I have tried so far, a face-to-face conversation has been the most productive. Just sitting with someone over a cup of coffee has done wonders for me in terms of trying to understand what their concerns are, what are the facts that I think people misunderstand about our research. Furthermore, I felt it helped the other side understand me as a scientist and a person, and no longer perceive me as one of the monsters we're supposed to be. They accepted that when we go into the lab, we do it with a true and honest belief that we are helping humankind, generating the knowledge necessary to further medical science. So I think there is a need, an increased need, to have this kind of interaction.

The reasons to engage are many. David mentioned some of them. First of all, it's really our obligation to the public. The public funds the research and if they have questions about the research we ought to explain to the public why we think the research is important and why we do it. And we have to understand that there may certainly be legitimate concerns about the welfare of animals in labs. We have to admit that people can come to us and ask reasonable questions. Are the animals suffering? How much are they suffering? Do they suffer all day long?

Another reason to engage in this kind of dialogue is misunderstanding from our opponents about scientific issues. There is a growing idea that the work is not valuable in advancing human health at all. It is easy to understand that acceptance of such a belief will lead many to believe the work is unethical. Instead they suggest that we engage in research for other reasons-because it brings money to the university, because it pays our salaries, and that's about it.

Getting the facts right on the science is critical if we are to have an ethical discussion. If we cannot agree on the scientific facts and benefits of the research it is extremely difficult to have a reasonable ethical discussion. But we must address the ethical issues as well, we must recognize there is a growing ethical concern about the work. As we heard, even if the research produces the benefits that we expect, there are some people who don't consider the work ethical. It doesn't matter that the animals were taken care of, it doesn't matter that we can produce new therapies and cures; they just don't think the research is ethical.

Both science and ethics must be core for the justification of animal research. In that sense, I agree that ethics is a big part of the debate. When we are challenged with explaining why the work is ethical, we can't just reply "all our research is done according to the regulations." That is not an ethical explanation, it is a legal one. We must instead articulate and put forward an ethical argument.

I believe the lack of dialogue did play some part in my case and the ongoing attacks at UCLA and elsewhere. The lack of dialogue favors the growth of extremism and violence. A small group of animal rights extremists has already decided that violence is the only way they are going to accomplish their social goals. They are taking the risks that go together with that stance. But there is more and more acceptance of the behavior of these individuals by others, because they perceive a lack of public dialogue on these issues. So I think dialogue can be very productive in easing these types of tensions too.

Who has to engage in dialogue? It is not just the scientists doing the work. Unless I give up science altogether, there is a limited amount of time that I can really devote to this outreach. I organized, with David, a panel at UCLA. It took months and months of organization just to have this one panel that lasted a couple of hours. Individual scientists cannot sustain an ongoing conversation with larger organizations that oppose animals in research. So it is up to all of us to engage in this dialogue - from the scientists to the physicians and vets and animal technicians and IACUC members. Institutional officials should be part of this dialogue as well, and scientific and professional societies, public health leaders and entities, and NIH. I do not believe NIH is merely a funding agency but that it has a responsibility to educate the population about public health and the science behind it. The institution must surely find the science to be sound and ethical, and they should be part of the conversation too, as well as patient advocacy groups, private medical foundations, and celebrities.

How do we encourage dialogue to happen? I think we are reaching a point where it would be extremely useful to consider putting effort into this; for example, an annual meeting-either NIH or Institute of Medicine-where people could come and voice their concerns and have an actual, honest dialogue. You're invited if you're really interested in having a dialogue-if we're just going to scream at each other, it's not going to be very effective. Such engagements can be useful because they release individual scientists and institutions from holding multiple, overlapping events on a regular basis. It is important to have a national stage where the public can voice their concerns about the research and where we can listen to them and see if there's anything valuable they are offering that would improve the welfare of animals in labs.

Another useful idea might be to create something like the Boyd Group in the UK, with representatives both pro [and opposed to] animal research who discuss specific ideas about how to advance the welfare of animals. Hosting these dialogues is a way institutions may help.

Another way to help is by funding such events. As an example, the one panel discussion we had at UCLA was pricey, with $80 \%$ of the funds going to security, and money was difficult to find. Just before this one event, some activists decided it was a good time to target my children at their school. So we were rushing around placing guards at elementary schools just to have this one event take place. But it did take place and it was a success. However, we cannot do this on a continuous basis. It is part of an educational mission of our institutions to have an ongoing, bidirectional dialogue.

Brian [Hare] yesterday told us that there is a growing concern about animal welfare, not just in labs but everywhere. 
The Discovery Channel, Animal Planet, these are top moneymaking stations and show that the public is interested in and concerned about animal welfare. The same concern percolates into animal research. There's so much we can tell them about the welfare of the animals that I think will satisfy most of them.
I cannot be certain that dialogue will work. But at the very least if we do engage in dialogue, I believe there's a chance to make progress. Of course there is no way to control those who decide to plant bombs. But perhaps it will make the life of those small groups that advocate violence, and those that silently approve, much more difficult.

\section{Standing Up for Science: Discussion}

Questioner: Thank you very much for your presentations today. I want to encourage scientists to contact their state biomedical research associations, to volunteer as a speaker. We provide training and we encourage dialogues and we go into the classroom and talk to the kids. There are a lot of programs that are doing science cafés to talk to the public as well as teachers and students.

Questioner: Our organization has a very strong position against the use of violence to promote any kinds of goals. We have been very public about that stance and I just want to reiterate that here. Again, I want to bring up that we weren't part of the discussion and I appreciate the reference to that. "Standing Up for Science" kind of makes it sound like if you don't support everything that everyone's doing in animal research, you don't stand for science. Our organization promotes the Three Rs. We have a pain and distress initiative, where we try to end pain and distress in animals. We also seek replacements. I think we're all supposed to be working toward replacement and I don't want it to be that we're against science because we want to make changes in welfare. Finally, I totally agree with the idea of dialogue. I would love to talk to you about how to do that [and about] next steps. I wouldn't agree with having a meeting at NIH on an annual basis. The animal welfare department of NIH didn't invite us to this meeting so I'd want to be on more neutral ground if we did that. But I love the idea of a Boyd Group in the United States. I think that's a fantastic idea and would like to see that move forward.

Dr. Ringach: I want to reply to some things. I know your organization statement about violence and I welcome that. But as you know, many other organizations, very large organizations, do not have such a statement. I actually implicitly understand the violence because activists are frustrated. [But] if we are going to have an honest discussion about animal research, it becomes problematic if you cannot hear my voice and that of my colleagues. You might have realized that the only scientists speaking up are the ones that already found bombs under their cars or their children have been targeted. So your statement is welcome, but my question to you is, What are you doing to prevent this in the future? And if we were to engage in dialogue, how are you going to stop these people?

Questioner: We have worked over the last 10 years trying to engage in dialogue, for example reaching out to IACUCs and saying "We want to work with you on pain and distress. Let's come together, find common ground." We come out against violent activity, we're very strongly opposed to it [and] have made that clear. We come out, we do letters to the editor. I think engaging in dialogue will naturally push [violent] activity aside. What else could we do other than try to sit down and have a discussion...? What else would you propose that we do?

Dr. Ringach: For example, I recall one instance when [the home of] one of my colleagues at UC Santa Cruz was bombed in the middle of the night when his family was sleeping there. I recall that the Humane Society contributed $\$ 1500$, I think, as a reward-the same amount offered to find, I don't know, whoever stepped over a squirrel-that kind of thing....

Dr. Collins: Clearly dialogue is the issue here, [but] this may not necessarily be the best forum for this dialogue to occur. We have other people that would like to ask questions.

Dr. Ringach: I can meet with you and talk about the possibilities.

Questioner: That sounds great.

Questioner: I'm here as a compliance official and as a scientist, but most importantly I'm here as a parent. I make sure when I'm with my children to reiterate the importance of animal welfare, and I want to thank everybody here because now I have even more things to go home and talk to them about in terms of animal welfare and how it is really important to the science that we're doing and to advances that we have [made and supported].... The question I have is about talking to upper administrators and IOs and such. How do we approach them about being staunch advocates and really defending the animal research that we're doing at our institutions? What would you suggest?

Dr. Jentsch: The important aspect of the message that I would give you is to think about the way we started the discussion today and yesterday, not so much the way we ended the discussion today. There's a tremendous positive message about biomedical research and about the efforts by scientists and administrators and students engaged in this effort. What more positive message could you give to the broader public about the coordinated, academically honest research endeavors of the membership of the university? The administration should be making every effort to tell the community about its 
discoveries and its process and the brilliant people that work in its labs, rather than being in the situation some universities find themselves in of being called upon only when somebody's being targeted, only when someone's decided they don't like the academically justifiable research of a faculty member. The message to take home is that we have exciting, interesting research-that probably people like you know more about because in the IACUC you [see] what's going on in the university. I know what I'm doing, but you know the bigger picture. Take those messages home and work with your administration and make sure they see research as the positive thing it is. The problem-I'll say it again-is that important research breeds malcontent. It always will. That's fundamentally tethered to the fact that it's exciting, it's breaking ground. We have to be willing to send that message to the public, despite the loud minority of voices that try to drown it out. I think that's the way. Go home and say, "Let's talk about excellent research, about the way we conduct research, about the methods and the facilities and the procedures and the administration and the way we do this."

Questioner: I oppose animal research on ethical grounds. However, I do think there's something we can agree on. I'm not going to stand here and tell you that I think no animal research is applicable to human health. And I don't think anyone up there or in the audience would be able to tell me that there is no such thing as reckless or frivolous animal research. My question-I guess it's a suggestion-is, Is anything being done to root out and discourage reckless and frivolous animal research? Because it's something that would certainly appease what you would call radical animal rights groups and would also reflect a better perception of your industry.

Dr. Jentsch: I suggest that this 2-day event answers your question. We see people who sat here to the very end of the session whose daily lives are spent on this basic point. IACUC administrators, laboratory animal medicine veterinarians - this is what they do. It's their job to look into the conduct of research to ensure its quality. That said, I want to ask everybody to back up for a second. I might pull this pen out of my pocket and say, "Damn this pen, it leaked in my pocket. Pens are bad!" But of course, there are about 120 other pens in this room that did exactly what they are supposed to do for the last 2 days. The pen that bled in my pocket was not the story. The 120 pens that did exactly what they were supposed to do for the last 2 days and followed the rules are the real story. [applause] Unfortunately that's not the way things are broadcast.

Questioner: May I respond? Until you point out the frivolous and reckless research and you say this is the kind of thing that should not be done, it appears that you are protecting the industry and not the research itself.
Questioner: I had the good fortune to spend 2 decades at NIH. The comment I want to make is, We all came here to celebrate 25 years of the Public Health Service Policy and the efforts and strides forward that we've made, [to celebrate] where we are today and where we hope to go in the future. Many people could not be on the podium because 2 days could not begin to cover even a small percentage of that story. I applaud the efforts of the committee that made the decisions [about speakers for these 2 days].

Questioner: I want to go back to a comment about what the university can do to promote research and how to promote the researchers. When the attacks against UCLA occurred, the media communication really struggled with what to do. One of the thoughts was, in press releases, not to include the species in the important research. Of course, we shot that idea down and we're doing quite the opposite. In all of our press releases, we are specifically naming the animal model that was used. So far, it's worked. I recommend that you make sure that your own media communications do the very same thing. [applause]

Questioner: First, I want to say that we, too, have a position statement against any kind of violence and that it's against what we as an organization are for. We're for humane treatment of animals and that includes the human animal as well as the nonhuman. Unfortunately there isn't a way that we're able to root out the concern and I think that's why there's a bit of frustration. The other [thing I want to say] is, you talked about fear in your presentation and I think that's part of the problem that affects all of us. I think those in research have a fear of ever identifying any wrongdoing because of how that may be used and waved around, so there isn't action taken perhaps when it should be. I also think there is fear among those who want to do the right thing and recognize problems but [who fear that], if they speak up, they might lose their funding, they might lose their position-there's a history of action taken against those who are outspoken. But I do believe that there is a middle ground and that dialogue is possible. And that we do have to have balance in bringing that dialogue together and a willingness and recognition of the wrongs that are out there and a serious effort to address them.

Dr. Collins: It appears that we have come to the end of our 2-day (for some of us, 3-day) session. We gathered together to celebrate the system that was created to take care of the animals, to assess the appropriateness of the work being done, and to move forward in the improvement of human health. I thank all of you for the contributions you have made to the discussions. [I am also grateful] for our opportunity to gather information in a way that has not been done before [... and for] the steps that have been taken in the last 25 years to improve the health of animals, to advance science, and to advance legitimate concerns about the welfare of research animals. 


\section{ANIMAL WELFARE AND SCIENTIFIC RESEARCH: 1985 TO 2010 \\ October 25, 2010 | Animal Welfare}

\section{P ROG R A M}

7:00am-8:30am

7:00am-8:30am

8:30am-8:45am

8:45am-9:00am

9:00am-10:10am

10:10am-10:30am

10:30am-10:50am

10:50am-12:00noon

12:00noon-12:20pm

$12: 20 \mathrm{pm}-1: 20 \mathrm{pm}$
Registration (FOYER, SALON D)

Continental Breakfast (SALON E-H)

Welcome (SALON A-D)

Patricia Brown, Office of Laboratory Animal Welfare, NIH

Chester Gipson, Animal Care, Animal and Plant Health Inspection Service, USDA

\section{Introduction (SALON A-D)}

Nelson Garnett, Office of Laboratory Animal Welfare, NIH, retired

Animal Housing Facilities (SALON A-D)

Moderator: Taylor Bennett, National Association for

Biomedical Research

Where We Came From - Betty Goldentyer, Animal Care, Animal and Plant Health Inspection Service, USDA and Christian Newcomer, Association for Assessment and Accreditation of Laboratory Animal Care International

How We Got There - Lauretta Gerrity, University of Alabama and Willie McCullough, National Center for Research Resources, NIH

Where We Are Today - Steven Leary, Washington University

Discussion (SALON A-D)

Break

Institutional Animal Care and Use Committees (SALON A-D) Moderator: James Taylor, Office of Animal Care and Use, NIH, retired Formative Years - Ralph Dell, Institute for Laboratory Animal Research, retired

Best Practices - Kathryn Bayne, Association for Assessment and Accreditation of Laboratory Animal Care International

Future - Jerry Collins, Yale University and Office of Laboratory Animal Welfare, NIH

\section{Discussion (SALON A-D)}

\section{Lunch (SALON E-H)}

Continuing Education

Day one of the Symposium was approved for 6.5 hours AAVSB RACE credits and the Keynote Address for 1.0 hours AAVSB RACE credits. 
1:20pm-2:15pm

2:15pm-2:35pm

2:35pm-2:55pm

2:55pm-4:25pm

4:25pm-4:45pm

4:45pm-5:15pm

5:15pm-6:30pm

6:30pm-8:30pm
Education and Training (SALON A-D)

Moderator: Mary Lou James, IACUC 101 Series

Conmed: The First Educational Forum - Steele Mattingly, University of Cincinnati, retired

Role of Education in Self-Evaluation - Susan Silk, Office of Laboratory Animal Welfare, NIH

\section{Discussion (SALON A-D)}

Break

\section{Veterinary Care (SALON A-D)}

Moderator: Patricia Brown, Office of Laboratory Animal Welfare, NIH

Where We Were - Gerald Van Hoosier, University of Washington, retired

Current Standard of Care and Technologies: Non Rodents Michael Talcott, Washington University

Current Standard of Care and Technologies: Rodents -

William White, Charles River Laboratories

Improving Animal Housing and Welfare -

Brian Hare, Duke University

\section{Discussion (SALON A-D)}

\section{Synergy of Working Together (SALON A-D)}

John Miller, Office for Protection from Research Risks, NIH, retired and Dale Schwindamin, USDA, retired

\section{Cocktails and Networking (FOYER, SALONS E)}

Keynote Address (SALON F-H)

\section{Standing on the Shoulders of Giants}

Charles McCarthy, Office for Protection from Research Risks, NIH, retired

"We are like dwarfs sitting on the shoulders of giants.

We see more, and things that are more distant, than they did, not because our sight is superior or because we are taller than they, but because they raise us up, and by their great stature add to ours." [John of Salisbury Metalogicon, 1159]

Panel Discussion (SALON F-H)

Reflections on IRAC and the U.S. Government Principles

Robert Whitney, PHS, retired and Thomas Wolfle, ILAR, retired

Dinner (SALON F-H)

Sponsored by AAALAC International 


\section{ANIMAL WELFARE AND SCIENTIFIC RESEARCH: 1985 TO 2010 \\ October 26, 2010 | Scientific Research}

P R O G A M

7:00am-8:30am

8:00am-9:00am

8:30am-8:35am

8:35am-8:45am

8:45am-9:15am

9:15am-9:45am

9:45am-10:00am

10:00am-10:15am

10:15am-10:45am

10:45am-11:15am

11:15am-11:45am

11:45am-12:00noon
Registration (FOYER, SALON D)

Continental Breakfast (SALON E-H)

Welcome (SALON A-D)

Susan Silk, Office of Laboratory Animal Welfare, NIH

Use of Animals in NIH Supported Biomedical Research (SALON A-D)

Sally Rockey, Office of Extramural Research, NIH

Global Impact of Animal Research on Infectious Diseases:

A CDC Perspective (SALON A-D)

Tanja Popovic, Centers for Disease Control and Prevention

Use of Animals in the Development of Medicines and Devices for Humans and Animals (SALON A-D)

David Jacobson-Kram, Food and Drug Administration

Discussion (SALON A-D)

Moderator: Susan Silk, Office of Laboratory Animal Welfare, NIH

Break

Animal Models and Basic Science: Bench to Bedside (SALON A-D)

Richard Nakamura, National Institute of Mental Health, NIH

Animal Models in Immunology and Transplant Medicine (SALON A-D)

Linda Cendales, Emory University

Animal Models Facilitate Rapid Responses to Emerging Infectious Diseases (SALON A-D)

Michael Kurilla, National Institute of Allergy and Infectious Diseases, NIH

Discussion (SALON A-D)

Moderator: Richard Nakamura, National Institute of Mental Health, NIH 
12:00noon-1:00pm

1:00pm-1:30pm

1:30pm-2:00pm

2:00pm-2:30pm

2:30pm-3:00pm

3:00pm-3:15pm

3:15pm-3:30pm

3:30pm-3:35pm

3:35pm-4:05pm

4:05pm-4:45pm

4:45pm-5:15pm
Lunch (SALON E-H)

Challenges of an Aging Brain (SALON A-D)

Stuart Zola, Yerkes National Primate Research Center

Genetic Vulnerabilities Associated with Cancer and Aging (SALON A-D)

Nancy Hopkins, Massachusetts Institute of Technology

Toward a Better Understanding of Depression, Schizophrenia, and Autism (SALON A-D)

Larry Young, Yerkes National Primate Research Center

Insight into Brain Development from Animal

Vocalization Studies (SALON A-D)

Erich Jarvis, Duke University

Discussion (SALON A-D)

Moderator: Stuart Zola, Yerkes National Primate Center

Break

Introduction (SALON A-D)

Jerry Collins, Yale University and Office of Laboratory

Animal Welfare, NIH

Research Animal Welfare: Looking Backward to Looking Forward (SALON A-D)

Susan Lederer, University of Wisconsin

Standing up for Science (SALON A-D)

Ways Individual Scientists and Clinicians Can Support Animal Research

J. David Jentsch, University of California, Los Angeles

The Need for Public Dialogue

Dario Ringach, University of California, Los Angeles

Panel Discussion (SALON A-D)

Moderator: Jerry Collins, Yale University and Office of Laboratory Animal Welfare, $\mathrm{NIH}$

Continuing Education

Day two of the Symposium was approved for 6.5 hours AAVSB RACE credits. 


\section{ANIMAL WELFARE AND SCIENTIFIC RESEARCH: 1985 TO 2010}

\section{Speaker List}

Kathryn Bayne, PhD, DVM, DACLAM

Global Director, Association for Assessment and

Accreditation of Laboratory Animal Care International

\section{B. Taylor Bennett, DVM, PhD}

Senior Scientific Advisor,

National Association for Biomedical Research

\section{Patricia A. Brown, VMD, MS, DACLAM}

Director, Office of Laboratory Animal Welfare, National Institutes of Health

\section{Linda Cendales, MD}

Assistant Professor of Surgery, Division of Plastic and Reconstructive Surgery and Division of Transplantation, Department of Surgery, Emory University School of Medicine and Director, Vascularized Composite Allotransplantation and the Laboratory of Microsurgery, Emory Transplant Center

\section{Jerry Collins, PhD}

Professor of Anesthesiology, Yale University School of Medicine and IPA, Division of Policy and Education, Office of Laboratory Animal Welfare,

National Institutes of Health

Ralph B. Dell, MD

Director, Institute for Laboratory Animal

Research, retired

\section{Nelson L. Garnett, DVM, DACLAM}

Office of Laboratory Animal Welfare,

National Institutes of Health, retired

\section{Lauretta W. Gerrity, DVM}

Associate Vice President for Research Operations and Compliance and Professor, Division of Comparative Medicine, Department of Genetics, University of Alabama

\section{Chester A. Gipson, DVM}

Deputy Administrator, Animal Care,

Animal and Plant Health Inspection Service,

U.S. Department of Agriculture

\section{Betty J. Goldentyer, DVM}

Eastern Regional Director, Animal Care, Animal and Plant Health Inspection Service, U.S.

Department of Agriculture
Brian Hare, PhD

Assistant Professor of Evolutionary Anthropology,

Duke Institute for Brain Sciences

\section{Nancy Hopkins, PhD}

Professor of Biology, Department of Biology, Massachusetts Institute of Technology

\section{David Jacobson-Kram, PhD, DABT}

Associate Director for Pharmacology and Toxicology, Office of New Drugs, Food and Drug Administration

\section{Mary Lou James, BA, LATG}

Consultant, Regulatory Compliance, Research Animal Welfare and President, IACUC 101 Series

\section{Erich D. Jarvis, PhD}

Associate Professor, Howard Hughes Medical Institute, Department of Neurobiology, Duke University Medical Center

\section{J. David Jentsch, PhD}

Professor, Departments of Psychology and Psychiatry, David Geffen School of Medicine,

University of California, Los Angeles. Associate

Director for Research, Brain Research Institute

Michael G. Kurilla, PhD, MD

Director, Office of BioDefense Research Affairs and Associate Director, BioDefense Product Development, Division of Microbiology and Infectious Diseases, National Institute of Allergy and Infectious Diseases, National Institutes of Health

\section{Steven L. Leary, DVM}

Assistant Vice Chancellor for Veterinary Affairs and Director, Division of Comparative Medicine, Washington University School of Medicine

\section{Susan E. Lederer, PhD}

Robert Turell Professor, Medical History and Bioethics, University of Wisconsin 


\section{ANIMAL WELFARE AND SCIENTIFIC RESEARCH: 1985 TO 2010}

\section{Speaker List}

Steele F. Mattingly, DVM

Director, Laboratory Animal Medical Services,

University of Cincinnati, retired

\section{Charles R. McCarthy, PhD}

Office of Education and Compliance Oversight, Virginia Commonwealth University. Director, Office for Protection from Research Risks,

National Institutes of Health, retired

Willie D. McCullough, PhD

Senior Program Official, Office of Construction Grants, National Center for Research Resources,

National Institutes of Health

John G. Miller, DVM, ACLAM

Director, Office for Protection from Research Risks,

National Institutes of Health, retired

\section{Richard K. Nakamura, PhD}

Scientific Director, Division of Intramural Research Programs, National Institute of Mental Health, National Institutes of Health

\section{Christian E. Newcomer, VMD, DACLAM}

Executive Director, Association for Assessment and Accreditation of Laboratory Animal Care International

Tanja Popovic, MD, PhD

Deputy Associate Director for Science,

Centers for Disease Control and Prevention

\section{Dario Ringach, PhD}

Professor, Neurobiology and Psychology, Jules Stein Eye Institute, Biomedical Engineering Program, David Geffen School of Medicine, University of California, Los Angeles

\section{Sally J. Rockey, PhD}

Deputy Director for Extramural Research and Director, Office of Extramural Research,

National Institutes of Health

\section{Dale Schwindaman, DVM}

Deputy Administrator, Regulatory Enforcement and Animal Care, Animal and Plant Health Inspection Service, U.S. Department of Agriculture, retired
Susan B. Silk, MS

Director, Division of Policy and Education, Office of Laboratory Animal Welfare, National Institutes of Health

\section{Michael R. Talcott, DVM, DACLAM}

Director, Veterinary Surgical Services, Division of Comparative Medicine and Research Assistant Professor, Surgery and Neurosurgery,

Washington University

\section{James F. Taylor, DVM}

Director, Office of Animal Care and Use, National Institutes of Health, retired

\section{Gerald L. Van Hoosier, DVM, DACLAM}

Professor Emeritus, Comparative Medicine, School of Medicine, University of Washington

William White, VMD, MS, DACLAM, DECLAM

Corporate Vice President, Veterinary and

Professional Services, Charles River Laboratories

\section{Robert A. Whitney, DVM, MS}

Public Health Service, retired

\section{Thomas Wolfle, DVM, PhD}

Director, Institute for Laboratory Animal Research, National Research Council, National Academy of Sciences, retired

\section{Larry J. Young, PhD}

Professor, Department of Psychiatry and Behavioral Sciences, Emory University School of Medicine and Division Chief, Center for Behavioral Neuroscience, Yerkes National Primate Research Center

\section{Stuart M. Zola, PhD}

Director, Yerkes National Primate Research Center Professor, Psychiatry and Behavioral Sciences, Emory University School of Medicine and Associate Director, Emory Alzheimer's Disease Research Center 


\section{WELCOME}

Individuals engaged in biomedical research continually strive toward a common goalimproving human and animal health. Occasionally, it is important to come together to consider milestones in that progress. We are here today to recognize 25 years of improving animal welfare and advancing biomedical research. Animal Welfare and Scientific Research: 1985 to 2010 acknowledges Animal Welfare and Scientific Research, a symposium held by $\mathrm{NIH}$ in 1984 [see NIH Record, page 6]. Subsequent to that historic symposium, a number of important events occurred in 1985:

- The U.S. Government Principles for the Utilization and Care of Vertebrate Animals Used in Testing, Research and Training (U.S. Government Principles) were developed and promulgated by the Interagency Research Animal Committee (IRAC). All U.S. government agencies that use animals in research adopted the principles and conduct their animal activities in compliance with their guidance. [see U.S. Government Principles, page 10]

- The Health Research Extension Act of 1985 (HREA), "Public Law 99-158 Animals in Research" was passed. This law provides the statutory mandate for the implementation of the Public Health Service Policy on Humane Care and Use of Laboratory Animals (PHS Policy). Included in the HREA is the requirement for Institutional Animal Care and Use Committees (IACUC) to provide local oversight for PHS supported animal research activities.

- Amendments to the Animal Welfare Act were passed by Congress requiring the local review and oversight of animal research by IACUCs.

A symposium to acknowledge laws and policies does not sound like it would be very interesting. Yet an exciting and important story is woven into the fabric of the policies, guidelines, and regulations that guide oversight of the welfare of animals used in federallyfunded research. It is the story of the critical contribution of research animal models to the acceleration of biomedical discovery and to the improvement of human and animal health.

Fulfilling animal welfare protections according to the requirements of the PHS Policy, the U.S. Government Principles, and the Animal Welfare Act and Regulations is now, and has been for the last 25 years, a shared effort between the NIH, USDA, federally supported scientific investigators, and research institutions. These have been years of growth and learning [see Timeline, page 8]. OLAW, Animal Care, and IACUC 101 have joined together, in concert with support from federal agencies, NIH Institutes and Centers, and our non-profit and business colleagues to reflect on this milestone and consider the future. 


\section{ANIMAL WELFARE AND SCIENTIFIC RESEARCH: 1984 SYMPOSIUM}

The 2010 symposium will commemorate the first symposium on Animal Welfare and Scientific Research held by NIH in 1984. The 1984 symposium was the opening event in a wide-ranging education program on research animal welfare sponsored by the $\mathrm{NIH}$.

[NIH Record, April 24, 1984]

\section{The NIH Record}

U.S. Department of Health

and

Human Services
April 24

1984

Vol. XXXVI

No. 9
National
Institutes
of
Health

\section{Researchers, Animal Specialists, Activists Join In NIH Symposium on Proper Use of Animals}

\section{By Jim Doherty}

Some 450 biomedical researchers, laboratory animal specialists, ethicists, public officials and animal protection activists took part in a 2-day Symposium on Animal Welfare and Scientific Research in Washington, D.C., April 11-12.

The symposium was the opening event in a wide-ranging education program on research animal welfare sponsored by the National Institutes of Health. Goal of NIH's continuing education program is to combine both scientific and humane considerations in animal research.

NIH's Office of Protection for Research Risks planned the symposium and will direct the ensuing education program.

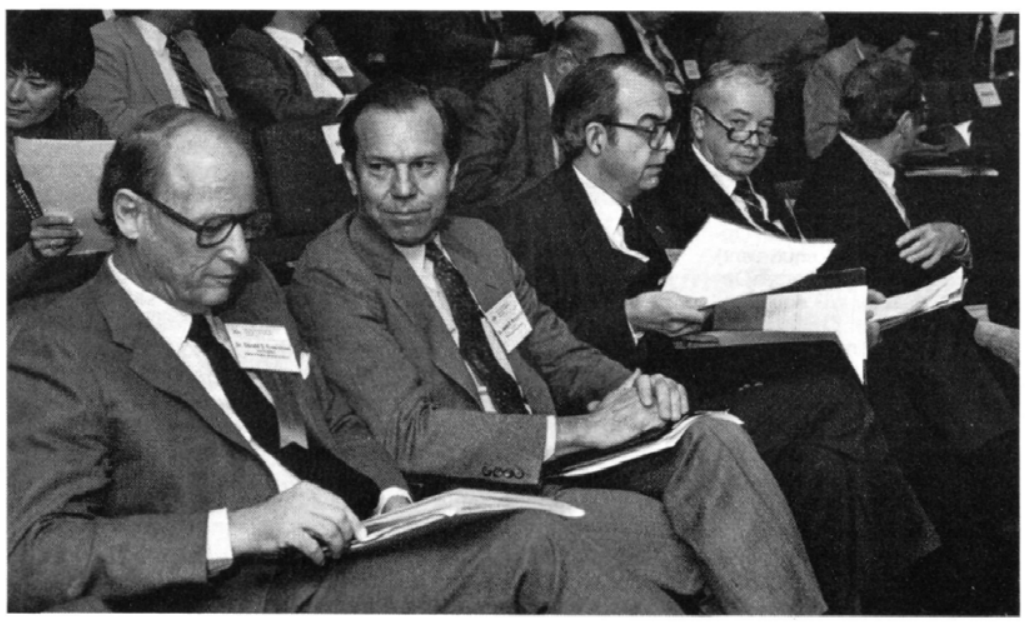

Symposium speakers at opening session: (I to r) Dr. Donald F. Fredrickson, former NIH Director who served as keynoter; Dr. James B. Wyngaarden, current NIH Director; Dr. Edward N. Brandt Jr., Assistant Secretary for Health; and Dr. Charles McCarthy, director of the Office for Protection from Research Risks, who served as symposium chairman. 


\section{ANIMALS}

Continued from Page 1

OPRR oversees procedures to ensure that Public Health Service award recipients who use animals in their research meet requirements of PHS policy on the humane care and use of animals.

In the symposium's opening session, Dr. James B. Wyngaarden, NIH Director, outlined procedures for approving NIH proposals and applications for projects involving animals and described current efforts to document the fact that PHS policies are implemented.

'The Federal Government cannot, on its own, adequately deal with the mounting pressure on this issue," he said. "It is the reponsibility of the institutions and investigators involved to become more active in responding to questions about the need for animals in research and appropriate

safeguards."

Dr. Edward N. Brandt Jr. Assistant Secretary for Health, told those attending: "The way we care for and use animals in research must be consistent with our lifesaving and life-protecting mission." $\mathrm{He}$ announced a proposed revision in the PHS Policy for Humane Care and Use of Animals and called for wide public comment.

In his keynote address, former NIH Director, Dr. Donald S. Fredrickson, reviewed some of the most striking modern medical advances that have depended on animals, including the heart-lung machine and viral vaccines-especially polio vaccine.

To make good public policy decisions on use of research animals, Dr. Fredrickson said, society needs to be familiar with sciensaid, society needs to be familiar with scientific methods and objectives; scientists need to provide convincing assurances that their methods are humane and responsible; and the whole community needs to seek an ethical frame "for judging the fruits of the quest for understanding in a fashion that does not retard the search itself."

The second session of the symposium opened with a discussion of proposed

changes in the PHS policy on animal welfare by Dr. William F. Raub, NIH Deputy Director for Extramural Research and Training. The for Extramural Research and Training. The proposed policy has been published as a
special edition of the NIH Guide for Grants and Contracts (Vol. 13, No. 5, Apr. 5, 1984). Dr. Raub explained that the new policy resulted, in part, from 10 site visits to randomly selected awardee institutions. It provides ad vantages to institutions that have approval from the American Association for Accreditation of Laboratory Animal Care (AAALAC)

Scientists are in the best position to promote good animal care practices, Dr. Raub said, and the vast majority are well motisaid, and the vast majority are well moti-
vated. The proposed changes will make easier to know exactly what is expected, he

said.

In a panel on clinical medicine moderated by television broadcaster John Charles Daly, Dr. Lawrence H. Cohn, director of Cardiac Surgery Research at Brigham and Women's Hospital, Boston, described a long series of cardiac surgery advances based on the use of large animals.

$\mathrm{He}$ presented his patient Theresa Rye.

R.N., who had open-heart surgery in 1982 at age 26 for a rare congenital heart defect called "scimitar syndrome," which causes an

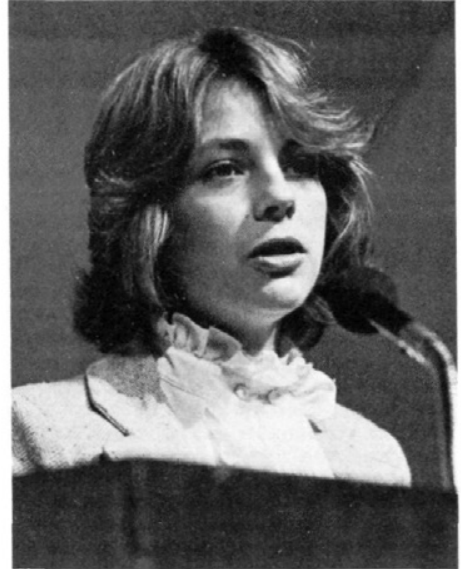

Ms. Rye of Boston, had her heart stopped for 30 minutes during an operation.

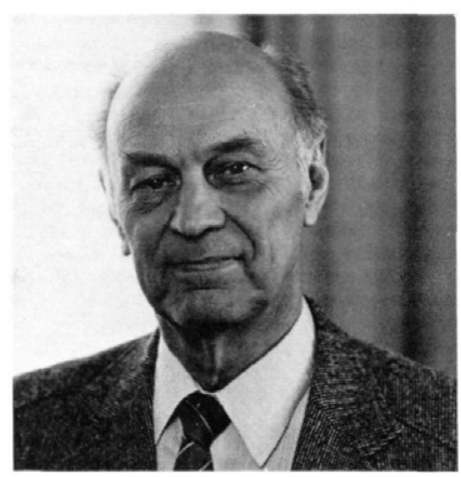

Dr. Karl Johan Öbrink of Uppsala, Sweden, summarized the animal symposium from an in ternational vlewpoint.

abnormal left-to-right shunting of blood

If not corrected, the condition leads inevitably to pulmonary artery hypertension and almost certain death.

My operation was unique," Ms. Rye said. "Part of my surgery required the heart-lung bypass machine to be turned off. It was a chilling experience for me to learn my body was frozen to 15 degrees centrigrade and that I had been clinically dead for 30 minutes during the operation.

"I hope that legislators, lobbyists, and research agencies appreciate that these kinds of procedures would be impossible had there not been an animal research model."

The role of animals in the development of transplantation was described by Dr. Stuart W. Jamieson, director of the Cardiac Surgery Experimental Laboratories at Stanford University Medical versity Medical Center. He presented his patient, Samuel S. Stark, who was diagnosed in 1980 as having primary pulmonary hyperten sion and by 1983 was near death.
Mr. Stark received a heart and lung transplant at Stanford on June 2, 1983. "After 53 days in the hospital and $2 \frac{1}{2}$ months as an outpatient, I'm now back home and working full time.

Dr. Earl H. Wood, emeritus professor of physiology and medicine at Mayo Medical School in Rochester, Minn., cited three examples of necessary human and animal experimentation from his own career: solving the blackout problem among fighter pilots in World War Il; solving the loss of oxygen content in arterial blood of the early astronauts; tent in arterial blood of the early astronauts:
and the development of a high speed and the development of a high speed computer-based tomographic system de-
signed to produce three-dimenstional noninvasive images of the heart, lung and blood in motion. The last two projects required animal models prior to human experiments

The final member of the panel, Dr. Clarence A. Rawlings, professor of small animal surgery at the University of Georgia School of Veterinary Medicine, illustrated the benefits of animal research for animals other than of anima

Congressman Doug Walgren $(\mathrm{Pa}$.) encouraged the scientific community to go forward under the leadership of the NIH in pursuit of high standards of laboratory animal care. In a panel chaired by Dr. Andrew N. Rowan, Tufts University School of Veterinary Medicine, varying views were presented on current measures of using and caring for research animals.

Dr. Constance Kagan of the Animal Political Action Committee described the approaches of three main groups in the animal proaches of three main groups in the animal
protection movement: those who are conprotection movement: those who are concerned about animal welfare; utilitarians who hold that each case of proposed animal use must be evaluated separately; and those who generally believe that the use of animals in research and testing should immediately cease.

Henry Spira, director of the Coalitions to Abolish the LD50 and Draize Tests, ex-

Abolish the LDSO a plained his activities as an attempt to attack "a universe of pain and death" at its most ulnerable spots.

Christine G. Stevens, president of the Animal Welfare Institute, presented arguments for strengthening the Animal Welfare Act. Dr. Karl Johan Obrink of the Biomedical Center, Uppsala, Sweden, summarized the symposium from an international viewpoint. $\mathrm{He}$ stressed that good animal care means better science, and that our knowledge of living things is still small, despite the recent progress. The only way to biomedical knowledge is to study life itself, he said. "Studies in an intact human or animal give you information that cannot be obtained in any other way. Studies at the various biological levels com. plement each other, and one level cannot give the same information as the other." Despite all laws and regulations on this subject, animal experimentation will always involve personal judgments of appropriateness, Dr. Obrink said. Some kind of review committee with scientific and nonscientific membership is necessary, he indicated.

There are two things we must resign ourselves to on pain of finding life unbearable; the ravages of time and human injustice. - Nicholas Chamfort

Page 11 


\section{THE History OF ANIMAL WELFARE POLICY, REGULATION, AND GUIDANCE}

\section{Timeline}

The history of animal welfare policy in the United States began well before 1985. The following timeline provides some of the key events that shaped or influenced the Health Research Extension Act of 1985, the Animal Welfare Act Amendments of 1985, and the U.S. Government Principles for the Utilization and Care of Vertebrate Animals Used in Testing, Research, and Training. The resulting authority from these laws and principles for oversight of animal care and use programs is vested in Institutional Animal Care and Use Committees (IACUC) to ensure the humane care and use of animals used in research.

\begin{tabular}{|c|c|}
\hline 1950 & First meeting of the Animal Care Panel. \\
\hline 1953 & Institute of Animal Resources (IAR) established within the National Research Council \\
\hline 1956 & IAR renamed Institute of Laboratory Animal Resources \\
\hline 1963 & $\begin{array}{l}\text { First edition of the Guide for the Care and Use of Laboratory Animals (Guide) } \\
\text { developed by the Animal Care Panel }\end{array}$ \\
\hline 1965 & $\begin{array}{l}\text { Incorporation of the American Association for the Accreditation of Laboratory Animal } \\
\text { Care (AAALAC) }\end{array}$ \\
\hline 1966 & $\begin{array}{l}\text { Congress passed the Laboratory Animal Welfare Act (PL 89-544) and the USDA was } \\
\text { named the responsible agency }\end{array}$ \\
\hline 1967 & $\begin{array}{l}\text { Animal Care Panel changed its name to the American Association for Laboratory } \\
\text { Animal Science (AALAS) }\end{array}$ \\
\hline \multirow[t]{2}{*}{1971} & $\begin{array}{l}\text { NIH Policy on Humane Care and Use of Laboratory Animals for PHS Supported } \\
\text { Institutions issued }\end{array}$ \\
\hline & USDA promulgated standards known as Subpart F, Stolen Animals (AWA) \\
\hline 1973 & $\begin{array}{l}\text { First Public Health Service (PHS) Policy on Humane Care and Use of Laboratory } \\
\text { Animals issued }\end{array}$ \\
\hline 1974 & Public Responsibility in Medicine and Research (PRIM\&R) established \\
\hline 1976 & Food and Drug Administration proposed Good Laboratory Practices (GLP) regulations \\
\hline \multirow[t]{3}{*}{1979} & $\begin{array}{l}\text { PHS Policy required each animal-using grantee institution to have a PHS Assurance } \\
\text { and a committee to maintain oversight of its animal care program }\end{array}$ \\
\hline & USDA promulgated standards known as Subpart E, Identification of Animals (AWA) \\
\hline & Good Laboratory Practices regulations became law \\
\hline 1982 & First PRIM\&R Animal Care and Use meeting held \\
\hline 1983 & $\begin{array}{l}\text { Memorandum of Understanding established among USDA, FDA and NIH relating to } \\
\text { laboratory animal care and welfare }\end{array}$ \\
\hline \multirow[t]{2}{*}{1984} & $\begin{array}{l}\text { Scientists Center for Animal Welfare established and held its first workshop in } \\
\text { Baltimore, MD }\end{array}$ \\
\hline & FDA proposes amendments to GLP regulations \\
\hline \multirow[t]{3}{*}{1985} & $\begin{array}{l}\text { U.S. Government Principles for the Utilization and Care of Vertebrate Animals Used in } \\
\text { Testing, Research and Training promulgated }\end{array}$ \\
\hline & Health Research Extension Act (P.L.99-158) passed by Congress \\
\hline & Animal Welfare Act Amendments passed by Congress \\
\hline \multirow[t]{2}{*}{1986} & Applied Research Ethics National Association (ARENA) established \\
\hline & $\begin{array}{l}\text { Animal Welfare Information Center (AWIC) established as part of the USDA National } \\
\text { Agricultural Library as provided in the } 1985 \text { AWA amendments }\end{array}$ \\
\hline
\end{tabular}




\begin{tabular}{|c|c|}
\hline & $\begin{array}{l}\text { Report of the American Veterinary Medical Association (AVMA) Panel on } \\
\text { Euthanasia published }\end{array}$ \\
\hline & PHS Policy revised to include the Health Research Extension Act \\
\hline 1987 & FDA issued final rule amending GLP regulations \\
\hline 1989 & $\begin{array}{l}\text { USDA promulgated regulations (known as Parts } 1 \text { and } 2 \text { ) implementing the } 1985 \\
\text { AWA Amendments }\end{array}$ \\
\hline \multirow[t]{2}{*}{1990} & $\begin{array}{l}\text { Office for Protection from Research Risks (OPRR) structure was changed to establish a } \\
\text { Division of Animal Welfare }\end{array}$ \\
\hline & $\begin{array}{l}\text { USDA promulgated standards known as Subpart B, Registration and Subpart C, } \\
\text { Research Facilities (AWA) }\end{array}$ \\
\hline 1991 & $\begin{array}{l}\text { USDA promulgated standards known as Part 3. In addition, amendments were made } \\
\text { to Part 2: Regulations in Subpart A, Licensing and Subpart D, Attending Veterinarian } \\
\text { and Adequate Veterinary Care (AWA) }\end{array}$ \\
\hline \multirow[t]{2}{*}{1992} & $\begin{array}{l}\text { First Institutional Animal Care and Use Committee Guidebook was developed by a } \\
\text { committee under the auspices of the Applied Research Ethics National Association } \\
\text { (ARENA) and OPRR }\end{array}$ \\
\hline & Animal Enterprise Protection Act of 1992 passed by Congress \\
\hline 1995 & $\begin{array}{l}\text { Memorandum of Understanding updated among USDA, FDA and NIH concerning } \\
\text { laboratory animal welfare }\end{array}$ \\
\hline \multirow[t]{2}{*}{1996} & $\begin{array}{l}\text { 7th Edition of the Guide for the Care and Use of Laboratory Animals revised by an } \\
\text { ILAR committee and published by the NRC }\end{array}$ \\
\hline & $\begin{array}{l}\text { AAALAC became the Association for the Assessment and Accreditation of } \\
\text { Laboratory Animal Care International }\end{array}$ \\
\hline 1997 & $\begin{array}{l}\text { National Air and Space Agency (NASA) released NASA Principles for the } \\
\text { Ethical Care and Use of Animals }\end{array}$ \\
\hline 1998 & IACUC 101, in conjunction with ARENA, debuted in Boston \\
\hline \multirow[t]{2}{*}{2000} & $\begin{array}{l}\text { OPRR Division of Animal Welfare was separated from OPRR and became the } \\
\text { Office of Laboratory Animal Welfare (OLAW), NIH }\end{array}$ \\
\hline & $\begin{array}{l}\text { Interagency Coordinating Committee on the Validation of Alternative Methods } \\
\text { (ICCVAM) designated as permanent interagency committee }\end{array}$ \\
\hline 2001 & 2000 Report of the AVMA Panel on Euthanasia published \\
\hline \multirow[t]{4}{*}{2002} & $\begin{array}{l}\text { ARENA/OLAW Institutional Animal Care and Use Committee Guidebook, Second } \\
\text { edition, published }\end{array}$ \\
\hline & $\begin{array}{l}\text { Animal Welfare Act Amendment modified the definition of animals to exclude rats, } \\
\text { mice, and birds bred for use in research }\end{array}$ \\
\hline & $\begin{array}{l}\text { Memorandum of Understanding established between the Veterans Administration } \\
\text { and NIH concerning laboratory animal welfare }\end{array}$ \\
\hline & $\begin{array}{l}\text { PHS Policy revised to permit verification of IACUC approval for competing } \\
\text { applications subsequent to peer review but prior to award }\end{array}$ \\
\hline 2006 & $\begin{array}{l}\text { Congress passed the Animal Enterprise Terrorism Act prohibiting the use of force, } \\
\text { violence, or threats for the purpose of damaging or interfering with the operations of } \\
\text { an animal enterprise }\end{array}$ \\
\hline 2007 & AVMA Guidelines on Euthanasia updated and renamed \\
\hline 2010 & $\begin{array}{l}\text { 8th Edition of the Guide for the Care and Use of Laboratory Animals revised by an } \\
\text { ILAR committee }\end{array}$ \\
\hline
\end{tabular}




\section{THE U.S. GOVERNMENT PRINCIPLES}

The U.S. Government Principles were promulgated in 1985 by the Interagency Research Animal Committee (IRAC) and adopted by U.S. Government agencies that either develop requirements for or sponsor procedures involving the use of vertebrate animals. The Principles were incorporated into the PHS Policy in 1986 and continue to provide a framework for conducting research in accordance with the Policy and are the foundation for humane care and use of laboratory animals in the United States.

\section{U.S. Government Principles for the Utilization and Care of Vertebrate Animals Used in Testing, Research, and Training}

The development of knowledge necessary for the improvement of the health and well-being of humans as well as other animals requires in vivo experimentation with a wide variety of animal species. Whenever U.S. Government agencies develop requirements for testing, research, or training procedures involving the use of vertebrate animals, the following principles shall be considered; and whenever these agencies actually perform or sponsor such procedures, the responsible Institutional Official shall ensure that these principles are adhered to:

I. The transportation, care, and use of animals should be in accordance with the Animal Welfare Act (7 U.S.C. 2131 et. seq.) and other applicable Federal laws, guidelines, and policies.*

II. Procedures involving animals should be designed and performed with due consideration of their relevance to human or animal health, the advancement of knowledge, or the good of society.

III. The animals selected for a procedure should be of an appropriate species and quality and the minimum number required to obtain valid results. Methods such as mathematical models, computer simulation, and in vitro biological systems should be considered.

IV. Proper use of animals, including the avoidance or minimization of discomfort, distress, and pain when consistent with sound scientific practices, is imperative. Unless the contrary is established, investigators should consider that procedures that cause pain or distress in human beings may cause pain or distress in other animals.

V. Procedures with animals that may cause more than momentary or slight pain or distress should be performed with appropriate sedation, analgesia, or anesthesia. Surgical or other painful procedures should not be performed on unanesthetized animals paralyzed by chemical agents.

VI. Animals that would otherwise suffer severe or chronic pain or distress that cannot be relieved should be painlessly killed at the end of the procedure or, if appropriate, during the procedure.

VII. The living conditions of animals should be appropriate for their species and contribute to their health and comfort. Normally, the housing, feeding, and care of all animals used for biomedical purposes must be directed by a veterinarian or other scientist trained and experienced in the proper care, handling, and use of the species being maintained or studied. In any case, veterinary care shall be provided as indicated.

VIII. Investigators and other personnel shall be appropriately qualified and experienced for conducting procedures on living animals. Adequate arrangements shall be made for their in-service training, including the proper and humane care and use of laboratory animals.

IX. Where exceptions are required in relation to the provisions of these Principles, the decisions should not rest with the investigators directly concerned but should be made, with due regard to Principle II, by an appropriate review group such as an institutional animal care and use committee. Such exceptions should not be made solely for the purposes of teaching or demonstration.

*For guidance throughout these Principles, the reader is referred to the Guide for the Care and Use of Laboratory Animals prepared by the Institute of Laboratory Animal Resources, National Academy of Sciences. 


\section{List of Abbreviations}

AAALAC Association for Assessment and Accreditation of Laboratory Animal Care [International]

AAAS American Association for the Advancement of Science

AALAS

AAVMC

American Association for Laboratory Animal Science

ACLAM

American Association of Veterinary Medical Colleges

ACUPO

American College of Laboratory Animal Medicine

AFIP

ALTBIB

AMP

APC

APHIS

APS

ASLAP

AV

AVMA

AWA

AWIC

BRL

CAAT

CDC

CIOMS

CITES

CITI

CT

DCE-MRI

DHEW

DHHS

DoD

ETS 123

Animal Care and Use Program Office (CDC)

Armed Forces Institute of Pathology

Bibliography on Alternatives to Animal

Testing (NLM)

Americans for Medical Progress

antigen-presenting cell

Animal and Plant Health Inspection Service

American Physiological Society

American Society of Laboratory Animal

Practitioners

attending veterinarian

American Veterinary Medical Association

Animal Welfare Act

Animal Welfare Information Center

Biological Resources Laboratory

Center for Alternatives to Animal Testing

Centers for Disease Control and Prevention

Council for International Organizations of

Medical Sciences

Convention on Trade in Endangered Species

of Wild Fauna and Flora

Collaborative Institutional Training Initiative

computed tomography

dynamic contrast enhanced magnetic

resonance imaging

Department of Health, Education, and

Welfare

Department of Health and Human Services

Department of Defense

European Convention for the Protection of

Vertebrate Animals Used for Experimental

and Other Scientific Purposes

FASEB Foundation of American Societies for

Experimental Biology

FBR Foundation for Biomedical Research

FDA Food and Drug Administration

GLP good laboratory practice(s)

HAART highly active antiretroviral therapy

HBV hepatitis B virus

HVAC heating, ventilation, and air conditioning

IACUC institutional animal care and use committee

IATA
$\mathrm{ICH}$

iiFAR

ILAR

IO

IPSC

IRAC

IRB

LAMA

LAWA

LAWTE

$\mathrm{MCI}$

micro-CT

MOU

MRI

NABR

NCRR

NCI

NHP

NIEHS

NLM

OACU

OLAW

OMB

OPRR

PAM

PCR

PET

PHS

PI

$\mathrm{PK} / \mathrm{PD}$

PPE

PRIM\&R

RACE

RFID

SARS

SCAW

SFI

SfN

SIV

SPF

SUBR

UIC

USDA

VA

WHO
International Conference on Harmonization

Incurably Ill for Animal Research

Institute for Laboratory Animal Research

Institutional Official

Interagency Primate Steering Committee

Interagency Research Animal Committee

institutional review board

Laboratory Animal Management Association

Laboratory Animal Welfare Act

Laboratory Animal Welfare Training

Exchange

mild cognitive impairment

microcomputed tomography

memorandum of understanding

magnetic resonance imaging

National Association for Biomedical

Research

National Center for Research Resources

(NIH)

National Cancer Institute (NIH)

nonhuman primate

National Institute of Environmental Health

Sciences

National Library of Medicine

Office of Animal Care and Use

Office of Laboratory Animal Welfare (NIH)

Office of Management and Budget

Office for Protection from Research Risks (NIH)

postapproval monitoring

polymerase chain reaction

positron emission tomography

Public Health Service

principal investigator

pharmacokinetics/pharmacodynamics

personal protective equipment

Public Responsibility in Medicine and

Research

Registry of Approved Continuing Education

radio frequency identification

sudden acute respiratory syndrome

Scientists Center for Animal Welfare

suggestion for improvement (AAALAC)

Society for Neuroscience

simian immunodeficiency virus

specific pathogen-free

States United for Biomedical Research

University of Illinois at Chicago

United States Department of Agriculture

US Department of Veterans Affairs (formerly

the Veterans Administration)

World Health Organization 


\section{Thanks to Institutional Supporters}

\section{ILAR Supporting Institutional Associates}

Charles River Laboratories, East Thetford, VT

National Institutes of Health Office of Animal Care and Use

National Research Council of Thailand

\section{ILAR Institutional Associates}

American Physiological Society

Bar-Ilan University

Baxter Healthcare Corporation

Bristol-Myers Squibb

Cardiovascular Research Foundation

City of Hope

CNIO (Centro Nacional de Investigaciones Oncológicas), Madrid

Cold Spring Harbor Laboratory

College of William and Mary

Columbia University

Cornell University

East Carolina University

Emory University

FDA Biosciences Library

Fiocruz Manguinhos

Genentech Inc.

Janssen Pharmaceutica NV

Lovelace Respiratory Research Institute

Maruzen International Co.

Massachusetts General Hospital

Mayo Clinic Jacksonville

\author{
Medaille College \\ Montana State University \\ New York State Department of Health, Albany \\ Ohio State University \\ Oregon Regional Primate Research Center \\ Osaka U Seimeikagaku \\ Pretoria University \\ Radboud Universiteit Nijmegen \\ Rush Presbyterian St. Luke's Medical Center \\ Sanofi-Aventis \\ St. Jude Children's Research Hospital \\ Sun Kyun Kwan University \\ Technologie Servier \\ Texas A\&M University \\ Tomkins-McCaw Library \\ Tufts University \\ UNAM Instituto de Biología \\ University of Cincinnati \\ University of Illinois at Chicago \\ University of Iowa \\ University of Minnesota \\ University of Notre Dame \\ University of Pennsylvania \\ University of Rochester \\ University of Tennessee, Memphis \\ University of Tokyo \\ University of Washington \\ USDA National Agricultural Library \\ Virginia Commonwealth University Library \\ Western University of Health Sciences \\ Walter Reed Army Institute of Research (WRAIR) Library
}




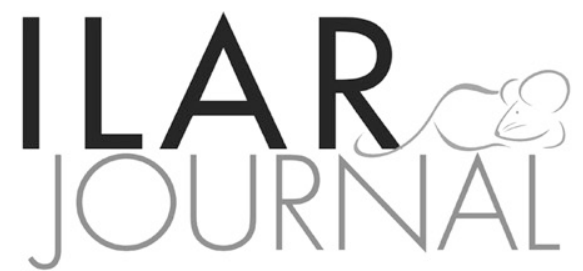

\section{INSTITUTE FOR LABORATORY ANIMAL RESEARCH}

NATIONAL ACADEMY OF SCIENCES

500 FIFTH STREET NW

WASHINGTON DC 20001 USA

PHONE: 2023342590 - FAX: 2023341687

ILARJ@NAS.EDU •WWW.NATIONAL-ACADEMIES.ORG/ILAR • FEDERAL TAX ID: 53-0196932

\section{ILAR Associate Program - ILAR Journal}

Renewal New
Member ID number

Requested beginning issue (if blank, subscription will begin with the next published issue)

Name

Organization

Street

City / State / Zip

Country

Please print or type if paying by credit card:

American Express

MasterCard

Visa

Discover

Cardholder name:

Credit card number:

Expiration date: Signature:

Or make check (US bank only) payable to National Academy of Sciences - ILAR. 


\section{${ }^{\text {* Recently Released * }}$}

\section{Guidance for the \\ Description of Animal Research in \\ Scientific Publications}

The publication of research articles involving animal studies, central to many disciplines in science and biomedicine, should include adequate and specific information to enable the interpretation, evaluation, and reproducibility of the reported study. Considerable variation in the amount of information required by scientific publications and reported by authors undermines this basic scientific need; insufficient information does not facilitate systematic reviews of animal studies and may result in the unnecessary use of animals, funding, and other resources in failed efforts to interpret and reproduce study results. This report provides clear explanations, examples, and supporting references for the effective reporting of all major components of animal research, including aspects of animal care and use that can affect research outcomes. The report includes sections on aquatic species.

To order your copy

contact National Academies Press:

online (www.nap.edu) or by telephone (888 6248373$)$ 


\section{ILAR Publications}

\section{Amphibians: Guidelines for the Breeding, Care and Management of Laboratory Animals}

This report of the Subcommittee on Amphibian Standards should serve as a useful guide to all users of amphibians, lead to success in the normal maintenance of amphibian colonies, and continue to stimulate efforts toward improving the quality of utilization of these animals. In addition to guidelines for animal care and quality, certain terminology is suggested. ISBN 0-309-07767-2; 1974, 162 pages, $6 \times 9$, paperbound

Animal Research in a Global Environment: Meeting the Challenges (Proceedings of the November 2008 International Workshop)

In 2008 ILAR convened a workshop that brought together 200 participants from 17 countries to identify and promote better understanding of important challenges in the conduct of animal research across country boundaries. These challenges include the sourcing of animals, the quality of veterinary care, competent staff, the provision of a suitable environment (including nutritious food and potable water) for animals, and ongoing oversight of the animal program, among others. This report summarizes the proceedings of the workshop, which has been a vital bridge for diverse colleagues and organizations around the world to advance initiatives to fill gaps in standards, professional qualifications, and coordination of animal use. ISBN 0-309-21502-1; 2011, 284 pages, $6 \times 9$, paperbound

\section{Chimpanzees in Research: Strategies for Their Ethical Care, Management, and Use}

Chimpanzees in biomedical and behavioral research constitute a national resource that has been valuable in addressing national health needs. However, the expected level of use of the chimpanzee model in biomedical research did not materialize, creating a complex problem that threatens both the availability of chimpanzees and the infrastructure required to ensure their well-being. This report examines the issues and makes recommendations. ISBN 0-309-05891-0; 1997, 108 pages, $6 \times 9$, paperbound

\section{Definition of Pain and Distress and Reporting Requirements for Laboratory Animals: \\ Proceedings of the Workshop Held June 22, 2000}

The goal of this ILAR/NIH joint workshop was to provide feedback from the scientific community to the USDA regarding the lack of a functional definition of "distress" as well as the efficacy of continuing to use current categories to report pain and distress. Speakers' areas of expertise and perspectives ranged from scientific research to animal welfare policy, protocol review, and relevant organizations or institutions. ISBN 0-309-0698-6; 2000, 132 pages, $6 \times 9$, paperbound

\section{The Development of Science-based Guidelines for Laboratory Animal Care: Proceedings of the November 2003 International Workshop}

The purpose of this workshop was to bring together experts from around the world to assess the available scientific knowledge that can affect the current and pending guidelines for laboratory animal care. Workshop presentations and discussions focused on identifying gaps in the current knowledge to encourage future research endeavors; assessing potential financial and outcome costs of nonscientifically based regulations, facilities, and research; and determining possible negative impacts of arbitrary regulations on animal welfare. ISBN 0-309-09302-3; 2004, 264 pages, $6 \times 9$, paperbound

\section{Education and Training in the Care and Use of Laboratory Animals}

Federal law requires that institutions provide training for anyone caring for or using laboratory animals. This volume provides the guidelines and resources needed to coordinate a quality training program, as well as to meet all legal requirements. ISBN 0-309-08691-4; 1991, 152 pages, $8.5 \times 11$, paperbound

\section{Guidance for the Description of Animal Research in Scientific Publications}

The publication of research articles involving animal studies, central to many disciplines in science and biomedicine, should include adequate and specific information to enable other researchers to interpret, evaluate, and reproduce a reported study. Considerable variation in the amount of information required by scientific publications and reported by authors undermines this basic scientific need; insufficient information does not facilitate systematic reviews of animal studies and may result in the unnecessary use of animals, funding, and other resources in failed efforts to interpret and reproduce study results. This report includes clear explanations, examples, and supporting references for the effective reporting of all major components of animal research, including aspects of animal care and use that can affect research outcomes. The report includes sections on aquatic species. ISBN 0-309-21951-5; 2011, 31 pages, online only 
Guide for the Care and Use of Laboratory

Animals, $8^{\text {th }}$ ed.

The Guide for the Care and Use of Laboratory Animals has been updated by a committee of experts to incorporate new scientific information on commonly used laboratory animals, including aquatic species, and provides extensive references. It is organized around major components of animal use: key concepts of animal care and use; animal care and use program (including the roles and responsibilities of the institutional official, attending veterinarian, and the institutional animal care and use committee; regulatory considerations; program and personnel management; and program oversight); animal environment, husbandry, and management; veterinary care (including animal procurement and transportation, preventive medicine, and clinical care and management); and physical plant design considerations.

\section{Guidelines for the Care and Use of Mammals in Neuroscience and Behavioral Research}

Expanding on the Guide for the Care and Use of Laboratory Animals, this report provides current best practices for animal care and use and discusses how the regulations and guidelines provided by the Guide, the Animal Welfare Act, the Animal Welfare Act Regulations, and PHS Policy can be applied to neuroscience and behavioral research. The report treats the development, evaluation, and implementation of animal-use protocols as a decision-making process, not just a decision. It encourages the use of professional judgment and careful interpretation of regulations and guidelines to develop performance standards that ensure animal well-being and high-quality research. This report is an indispensable resource for researchers, veterinarians, and institutional animal are and use committees. ISBN 0-309-08903-4; 2003, 224 pages. $6 \times 9$, paperbound

\section{Guidelines for the Humane Transportation of Research Animals}

Transporting research animals is a necessary part of the biomedical enterprise that can have substantial effects on the physiological and psychological condition of the animals. Individuals at research facilities often find arranging transportation of animals a challenge. In order to address a plethora of sometimes confusing and burdensome regulations pertaining to transportation of research animals, this report recommends that an interagency working group be established to coordinate federal inspections and permitting activities. It further recommends that steps be taken to ensure the availability of safe, reliable air and ground transportation for research animals. The report also establishes science-based good practices for transporting research animals and advises that research institutions designate a single individual to be responsible for ensuring safe shipment and receipt of animals. ISBN 0-309-10122-0; 2006, 160 pages, $6 \times 9$, paperbound

\section{Immunodeficient Rodents: A Guide to Their \\ Immunobiology, Husbandry, and Use}

This volume is an indispensable reference on the nature of immune defects in rodents and the special techniques necessary to maintain and breed them. The authors describe 64 inbred, hybrid, and mutant strains of rodents, each with some immune defect; explain mechanisms for ensuring genetic purity; and provide a standardized nomenclature for different varieties. ISBN 0-309-03796-4; 1989, 260 pages, $6 \times 9$, clothbound

\section{Infectious Diseases of Mice and Rats}

This edition-a must for all researchers who use these animals - provides practical suggestions for breeding, keeping, and identifying pathogen-free laboratory rodents. ISBN 0-309-06332-9; 1991, 415 pages, $6 \times 9$, paperbound

\section{Companion Guide to Infectious Diseases of Mice and Rats}

This companion to Infectious Diseases of Mice and Rats makes practical information on rodent diseases readily accessible to researchers. ISBN 0-309-04283-6; 1991, 108 pages, $6 \times 9$, paperbound

\section{International Perspectives: The Future of Nonhuman Primate Resources, Proceedings of the Workshop Held April 17-19, 2002}

Nonhuman primates (NHP) continue to play an important role in the research of many human diseases such as malaria and AIDS. Changes in the need for different species of NHP, the adequacy of the current supply of NHP, and projections of future needs for NHP are issues that concern scientists, veterinarians, and funding authorities from countries that are major users of NHP, as well as countries that produce and supply these animals. In this volume, workshop participant discussions relate to current shortfalls and excesses in NHP breeding and exportation programs, the status of breeding and conservation programs internationally, the development of specific pathogen-free colonies, difficulties in transporting NHP, and challenges in the management of NHP colonies. ISBN 0-309-08945-X; 2003, 262 pages, $6 \times 9$ paperbound

\section{Microbial and Phenotypic Definition of Rats and Mice: Proceedings of the 1998 US/Japan Conference}

This workshop is part of a long-term program to nurture international collaborative and information-exchange activities. As genetics and genomics affect the study of biology and medicine, the role of comparative medicine cannot be understated. Workshop contributors seek to enhance the genetic and microbiologic integrity of laboratory rat and mice colonies worldwide. The mouse has been a critical model for the discovery of genes responsible for several cancers and many other diseases. The rat model "functionally" characterizes mammalian model systems. This meeting sought to help global scientific enterprise harmonize the mouse and rat models and to meet the research challenges of the 21st century. ISBN 0-309-07389-8; 1999,110 pages, $6 \times 9$, paperbound

\section{Monoclonal Antibody Production}

Monoclonal antibodies are important reagents used in research, diagnosis, and treatment of diseases. They are produced by 
injection into the abdominal cavity of a suitably prepared mouse or by tissue culturing cells in plastic flasks. This report weighs the costs and benefits of each method and makes recommendations for their uses. ISBN 0-309-07511-4; 1999,74 pages, $6 \times 9$, paperbound

\section{National Need and Priorities for Veterinarians in Biomedical Research}

This report identifies various factors that contributed to creating an unfulfilled need for veterinarians in the biomedical research workforce, including an increase in the number of $\mathrm{NIH}$ grants utilizing animals and the burgeoning use of transgenic rodents, without a comparable change in the supply of appropriately trained veterinarians. The committee developed strategies for recruiting more veterinarians into careers in biomedical research. ISBN 0-309-09083-0; 2004, 102 pages, $6 \times 9$, paperbound

Nutrient Requirements of Laboratory Animals, 4th ed. In the years since the third edition of this indispensable reference was published, a great deal has been learned about the nutritional requirements of common laboratory species: rat, mouse, guinea pig, hamster, gerbil, and vole. The fourth edition presents the current expert understanding of the lipid, carbohydrate, protein, mineral, vitamin, and other nutritional needs of these animals. The extensive use of tables provides easy access to a wealth of comprehensive data and resource information. ISBN 0-309-05126-6; 1995, 192 pages, $8.5 \times$ 11, paperbound

\section{Occupational Health and Safety in the Care and Use of Nonhuman Primates}

The field of occupational health and safety constantly changes, especially as it pertains to biomedical research. New infectious hazards are of particular importance at nonhuman-primate facilities. For example, the discovery that $B$ virus can be transmitted via a splash on a mucous membrane raises new concerns that must be addressed, as does the discovery of the Reston strain of Ebola virus in import quarantine facilities in the U.S. The risk of such infectious hazards is best managed through a flexible and comprehensive occupational health and safety program (OHSP) that can identify and mitigate potential hazards. This report is intended as a reference for vivarium managers, veterinarians, researchers, safety professionals, and any other persons who are involved in developing or implementing an OHSP dealing with nonhuman primates. This report attempts to list the important features of an OHSP and provide the tools necessary for informed decision-making in developing an optimal program that meets all particular institutional needs. ISBN 0-309-08914-X; 2003, 184 pages, $6 \times 9$, paperbound

\section{Occupational Health and Safety in the Care and Use of Research Animals}

Much has been written about the care of research animals, yet little guidance has appeared on protecting the health and safety of the people who care for or use these animals. This report, an implementation handbook and companion to the Guide, identifies principles for building a program and discusses the accountability of institutional leaders, managers, and employees for a program's success. ISBN 0-309-05299$8 ; 1997,168$ pages, $6 \times 9$, paperbound

\section{The Psychological Well-Being of Nonhuman Primates}

A 1985 amendment to the Animal Welfare Act requires those who keep nonhuman primates to develop and follow appropriate plans for promoting the animals' psychological wellbeing. The amendment, however, provides few specifics. The Psychological Well-Being of Nonhuman Primates recommends practical approaches to meeting those requirements. ISBN 0-309-10359-2; 1998, 184 pages, $6 \times 9$, paperbound

\section{Recognition and Alleviation of Distress in Laboratory Animals}

The use of animals in research adheres to scientific and ethical principles that promote humane care and practice. Scientific advances in our understanding of animal physiology and behavior often require theories to be revised and standards of practice to be updated to improve laboratory animal welfare. The new report from the Institute for Laboratory Animal Research (ILAR), Recognition and Alleviation of Stress and Distress in Laboratory Animals, focuses on the stress and distress experienced by animals used in research. It aims to educate laboratory animal veterinarians; students, researchers and investigators; Institutional Animal Care and Use Committee members; animal care staff and animal welfare officers on the current scientific and ethical issues associated with stress and distress in laboratory animals. The report evaluates pertinent scientific literature to generate practical and pragmatic guidelines, focusing specifically on the following areas: the scientific understanding of causes and functions of stress and distress; the transformation of stress to distress; and the identification of principles for the recognition and alleviation of distress. Moreover, the report discusses the role of humane endpoints in situations of distress and principles of minimization of distress in laboratory animals. Finally, the report identifies areas in which further scientific investigation is needed to further improve laboratory animal welfare. ISBN 0-309-10817-9; 2008, 136 pages, $6 \times 9$, paperbound

\section{Recognition and Alleviation of Pain in Laboratory Animals}

Recognition and Alleviation of Pain in Laboratory Animals, the second of two reports revising the 1992 publication Recognition and Alleviation of Pain and Distress in Laboratory Animals, focuses on pain experienced by animals used in research. This book aims to educate laboratory animal veterinarians; students, researchers, and investigators; institutional animal care and use committee members; and animal care staff and animal welfare officers about the current scientific and ethical issues associated with pain in laboratory animals. The committee evaluated pertinent scientific literature to generate practical and pragmatic guidelines for recognizing 
and alleviating pain in laboratory animals, focusing on the following areas: physiology of pain in commonly used laboratory species; pharmacologic and nonpharmacologic principles to control pain; identification of humane endpoints; and principles for minimizing pain associated with experimental procedures. Finally, the report cites areas in which further scientific investigation is needed to improve laboratory animal welfare. ISBN 978-0-309-12834-6; 2009, 270 pages, $6 \times 9$, paperbound

Science, Medicine, and Animals, 2nd ed.

Science, Medicine, and Animals discusses how animals have been and continue to be an important component of biomedical research. It addresses the history of animal research and what it looks like today, and gives an overview of some of the medical advances that would not have been possible without animal models. Finally, it looks at the regulations and oversight governing animal use, as well as efforts to use animals more humanely and efficiently. ISBN 0-309-088941; 2004, 52 pages, $8.5 \times 11$, paperbound

\section{Science, Medicine, and Animals Teacher's Guide}

Science, Medicine, and Animals explains the role that animals play in biomedical research and the ways in which scientists, governments, and citizens have tried to balance the experimental use of animals with a concern for all living creatures. An accompanying Teacher's Guide is available to help teachers of middle and high school students use Science, Medicine, and Animals in the classroom. As students examine the issues in Science, Medicine, and Animals, they will gain a greater understanding of the goals of biomedical research and the real-world practice of the scientific method in general. The Teacher's Guide was reviewed by members of the National Academies' Teacher Associates Network and is recommended by the National Science Teacher's Association. ISBN 0-309-10117-4; 2005, 24 pages, $8.5 \times 11$, paperbound

\section{Scientific and Humane Issues in the Use of Random Source Dogs and Cats}

This report examines the value of random source animals in biomedical research funded by the National Institutes of Health (NIH) and the role of Class B dealers who acquire and resell live dogs and cats to research institutions. The report addresses (1) the important biomedical research questions and common research topics in contemporary $\mathrm{NIH}$-funded research where Class B dogs and cats are desirable/necessary as well as the frequency of these research topics (i.e., number of grants where the potential exists or the source of the animal is identified as a Class B dealer); (2) the specific characteristics (e.g., physiological, anatomical, or genetic) of the animals that make them particularly well suited for certain types of research; and (3) recommendations for the use of Class B dogs and cats. ISBN 978-0-309$13807-9 ; 2009$, 136 pages, $6 \times 9$, paperbound

\section{Strategies That Influence Cost Containment in Animal Research Facilities}

This second report of the National Research Council's Committee on Cost of and Payment for Animal Research presents the committee's conclusions and recommendations regarding cost containment methods for animal research facilities. This publication follows the Committee's initial report which examined interpretation of governmental policy (OMB Circular A-21) concerning institutional reimbursement for overhead costs of animal research facilities. ISBN 0-309-07261-1; 2000, 168 pages, 6 x 9, paperbound

\section{Use of Laboratory Animals in Biomedical and Behavioral Research}

Scientific experiments using animals have contributed significantly to the improvements of human health. Animal experiments were crucial to the conquest of polio, for example, and they will undoubtedly be one of the keystones in AIDS research. However, some persons believe that the cost to the animals is often high. Authored by a committee of experts from various fields, this report discusses the benefits that have resulted from animal research, the scope of animal research today, the concerns of advocates of animal welfare, and the prospects for finding alternatives to animal use. The authors conclude with specific recommendations for more consistent government action. ISBN 0-309-07878-4; 1988, 112 pages, $6 \times 9$, paperbound

\section{Laboratory Animal Management Series}

\section{Rodents}

Since ILAR (formerly the Institute of Laboratory Animal Resources) issued its last report on the general management of rodents, advances in biomedical technology and increased public awareness of laboratory animal issues have created a new research environment. This volume brings researchers up to date on both of these aspects of laboratory investigation and provides a comprehensive resource manual for management of laboratory rodents. ISBN 0-309-04936-9; 1996, 180 pages, $6 \times 9$, paperbound

\section{Dogs}

This revised edition incorporates the regulatory requirements and improved practices for laboratory animal care. ISBN 0-309-04744-7; 1994, 152 pages, $6 \times 9$, paperbound 


\section{ILAR Publications Order Form*}

\begin{tabular}{|c|c|c|c|c|}
\hline Title & Regular Price & $\begin{array}{l}\text { ILAR } \\
\text { Associate or } \\
\text { Web Order }\end{array}$ & Qty & Total \\
\hline & US \& Intl & US \& Intl & & \\
\hline Amphibians: Guidelines for the Breeding, Care and Management of Laboratory Animals & 35.25 & 31.73 & & \\
\hline Animal Research in a Global Environment: Meeting the Challenges (Proceedings) & 45.00 & 40.50 & & \\
\hline Chimpanzees in Research: Strategies for Their Ethical Care, Management, and Use & 21.75 & 19.57 & & \\
\hline Companion Guide to Infectious Diseases of Mice and Rats & 23.00 & 20.70 & & \\
\hline Definition of Pain and Distress and Reporting Requirements for Laboratory Animals & 30.00 & 27.00 & & \\
\hline Development of Science-based Guidelines for Laboratory Animal Care (Proceedings) & 55.95 & 50.36 & & \\
\hline Education and Training in the Care and Use of Laboratory Animals & 33.50 & 30.15 & & \\
\hline Guidance for the Description of Animal Research in Scientific Publications & 25.00 & 22.50 & & \\
\hline Guide for the Care and Use of Laboratory Animals, 8th ed. & 19.95 & 17.95 & & \\
\hline $\begin{array}{l}\text { Guide translations, ( } 7^{\text {th }} \text { ed): Chinese, French, Japanese, Korean, Portuguese, Russian, } \\
\text { Spanish, and Taiwanese }\end{array}$ & Contact NAP & Contact NAP & & \\
\hline Guidelines for the Care and Use of Mammals in Neuroscience and Behavioral Research & 19.95 & 17.95 & & \\
\hline Guidelines for the Humane Transportation of Research Animals & 42.00 & 37.80 & & \\
\hline Immunodeficient Rodents: A Guide to Their Immunobiology, Husbandry, and Use & 34.95 & 31.46 & & \\
\hline Infectious Diseases of Mice and Rats & 54.95 & 49.46 & & \\
\hline International Perspectives: The Future of Nonhuman Primate Resources (Proceedings) & 52.25 & 47.02 & & \\
\hline Laboratory Animal Management: Dogs & 32.95 & 29.66 & & \\
\hline Laboratory Animal Management: Rodents & 34.95 & 31.46 & & \\
\hline Microbial and Phenotypic Definition of Rats and Mice (Proceedings) & 26.25 & 23.63 & & \\
\hline Monoclonal Antibody Production & 23.00 & 20.70 & & \\
\hline National Need and Priorities for Veterinarians in Biomedical Research & 24.75 & 22.28 & & \\
\hline Nutrient Requirements of Laboratory Animals, 4th ed. & 37.95 & 34.16 & & \\
\hline Occupational Health and Safety in the Care and Use of Nonhuman Primates & 35.00 & 31.50 & & \\
\hline Occupational Health and Safety in the Care and Use of Research Animals & 45.95 & 41.36 & & \\
\hline Psychological Well-Being of Nonhuman Primates & 39.00 & 35.10 & & \\
\hline Recognition and Alleviation of Distress in Laboratory Animals & 35.95 & 32.36 & & \\
\hline Recognition and Alleviation of Pain in Laboratory Animals & 47.44 & 42.70 & & \\
\hline Recognition and Alleviation of Pain and Distress in Laboratory Animals & 34.75 & 31.28 & & \\
\hline Science, Medicine, and Animals, 2nd ed. & 8.95 & 8.05 & & \\
\hline Science, Medicine, and Animals, 2nd ed. Teacher's Guide & 4.50 & 4.05 & & \\
\hline Scientific and Humane Issues in the Use of Random Source Dogs and Cats & 35.00 & 31.50 & & \\
\hline Strategies That Influence Cost Containment in Animal Research Facilities & 42.00 & 37.80 & & \\
\hline Use of Laboratory Animals in Biomedical and Behavioral Research & 26.75 & 24.07 & & \\
\hline \multicolumn{5}{|c|}{ SUBTOTAL } \\
\hline \multicolumn{5}{|c|}{ Tax or GST (Residents of CA, DC, FL, MD, TX, and Canada) } \\
\hline \multicolumn{5}{|c|}{ Shipping and handling ( $\$ 4.50$ for first book, $\$ 0.95$ for each additional book) } \\
\hline & & & TOTAL & \\
\hline
\end{tabular}

*Many ILAR reports are available by chapter or book in PDF at the National Academies Press website: www.nap.edu. Prices subject to change.

Orders must be prepaid by check or money order in US dollars only, by credit card, or through a bona fide purchase order.

To order by mail: Return this form with payment to The National Academies Press, 500 Fifth Street NW, Lockbox 285, Washington, DC 20055. For credit card orders, call 1-888-624-8373 toll-free from the US or Canada, for other locations call 202-334-3313. For fax orders send to 202-334-2451. Order online at www.nap.edu.

Mention you are an ILAR Associate in order to receive your discount. Not an ILAR Associate yet? To learn about our Associates program and how to join, visit our website at www.national-academies.org/ilar.

Internet orders get a discount off the list price of most books. The ILAR Associate discount or the Internet discount may apply. Discounts cannot be combined.

I have enclosed a purchase order.

I have enclosed a check or money order for $\$$ payable to The National Academies Press.
Please charge my VISA/MasterCard/American Express

Cardholder's Name

Number Exp.

Signature

Please print.

Name

Address

City

Phone

State

Email 
* Recently Released *

\section{Animal Research in a Global Environment: Meeting the Challenges Proceedings of the November 2008 International Workshop}

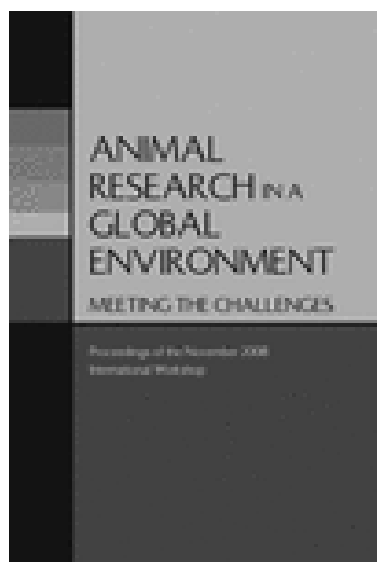

Animal research plays an essential role in efforts to meet increasing demands for global health care. Yet the animal research community faces negative impressions that industry and academia engage in international collaborations in order to conduct work in parts of the world where animal welfare standards are less stringent. Thus the importance of ensuring the international harmonization of the principles and standards of animal care and use cannot be overstated.

The Institute for Laboratory Animal Research (ILAR) convened a workshop that brought together 200 participants from 17 countries to identify and promote better understanding of challenges in the conduct of animal research across country boundaries. These challenges include the sourcing of animals, the quality of veterinary care, the provision of a suitable environment (including nutritious food and potable water) for animals, and ongoing oversight of the animal program, among others.

Animal Research in a Global Environment summarizes the proceedings of this workshop, whose impact has extended beyond the presentations made during the $21 / 2$ days of sessions. It has been a vital bridge for colleagues and organizations around the world to advance initiatives designed to fill gaps in standards, professional qualifications, and coordination of animal use.

To order your copy

contact National Academies Press:

online (www.nap.edu) or by telephone (888 6248373 ) 


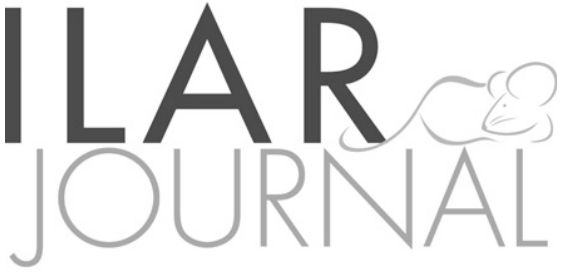

NATIONAL RESEARCH COUNCIL
InSTITUTE FOR LABORATORY ANimal Research

The National Academies

500 Fifth Street NW, Washington DC 20001

Phone: 202-334-2590; Fax: 202-334-1687

Email: ilarj@nas.edu; Website: http://www.nationalacademies.org/ilar

\section{ILAR Journal Current and Back Issues Order Form*}

Bulk pricing per issue: $1-10$ copies $\$ 54$ ea; $11-50$ copies $\$ 45$ ea; $51-100$ copies $\$ 40$ ea; $101+$ copies $\$ 30$ ea. Call ILAR for cost for out-of-print photocopies. *Prices above include US shipping charge, for international orders please add \$5 for 1st copy and \$1 for each additional copy ordered.

\begin{tabular}{|c|c|c|c|c|c|}
\hline Year & Issue & Topic & Quantity & Price ea & Total \\
\hline \multirow[t]{4}{*}{2011} & $52(\mathrm{~S})$ & $\begin{array}{l}\text { Proceedings of the Symposium on Animal Welfare and Scientific Research: } 1985 \text { to } 2010 \\
\text { (Please visit the ILAR Journal website for the complete list of presentations and related } \\
\text { documents from this symposium.) } \\
\text { - Introduction: By Ladies and Gentlemen, for Ladies and Gentlemen } \\
\text { - Animal Housing Facilities (4 presentations) } \\
\text { - Institutional Animal Care and Use Committees ( } 3 \text { presentations) } \\
\text { - Education and Training (2 presentations) } \\
\text { - Veterinary Care ( } 3 \text { presentations) } \\
\text { Synergy of Working Together } \\
\text { - Reynote Address: Standing on the Shoulders of Giants } \\
\text { Reflections on IRAC and the US Government Principles } \\
\text { Persectives of PHS Funding Agencies ( } 3 \text { presentations) } \\
\text { - Insighal Models and Basic Science-Bench to Bedside (3 presentations) } \\
\text { - Standing Up for Brience (2 presentations) }\end{array}$ & & & \\
\hline & $52(3)$ & $\begin{array}{l}\text { Animal Models of Drug Addictions: High Hopes for Therapeutic Treatments } \\
\text { - Introduction: Animal Models of Drug Addiction in Support of Novel Therapeutic Strategies } \\
\text { Translational Models of Interactions between Stress and Alcohol Consumption: } \\
\text { Strengths and Limitations } \\
\text { - Effects of Pre- and Neonatal Nicotine Exposure in Rodents: Inconsistent Evidence } \\
\text { Marijuana Dependence: Not Just Smoke and Mirrors } \\
\text { - Cracking the Molecular Code of Cocaine Addiction } \\
\text { - Mediating the Effects of Drug Abuse: The Role of Narp in Synaptic Plasticity } \\
\text { Aninging Mechanisms of Opiate Tolerance and Withdrawal during Early Development: } \\
\text { - Opioid Depels of the Human Experience } \\
\text { - Nucleus Accumbens Invulnerability to Methamphetamine Neurotoxicity } \\
\text { - The Neurobehavioral Pharmacology of Ketamine: Implications for Drug Abuse, } \\
\text { Addiction, and Psychiatric Disorders } \\
\text { - IACUC Perspective on Drug Addiction Research } \\
\text { - Workshop Summary: Neotropical Primates in Biomedical Research } \\
\text { A Glonizing Veterinary Training and Qualifications in Laboratory Animal Medicine: } \\
\text { A Globspective }\end{array}$ & & & \\
\hline & $52(2)$ & $\begin{array}{l}\text { Spineless Wonders: The Welfare and Use of Invertebrates in the Laboratory } \\
\text { and Classroom } \\
\text { - Introduction: Laboratory Invertebrates: Only Spineless, or Spineless and Painless? } \\
\text { - Invertebrate Models for Biomedical Research, Testing, and Education } \\
\text { - Culture and Maintenance of Selected Invertebrates in the Laboratory and Classroom } \\
\text { - Invertebrate Resources on the Internet } \\
\text { - Anesthesia, Analgesia, and Euthanasia of Invertebrates } \\
\text { Pain and Suffering in Invertebrates? } \\
\text { - Nociceptive Behavior and Physiology of Molluscs: Animal Welfare Implications } \\
\text { Philosophical Background of Attitudes toward and Treatment of Invertebrates } \\
\text { IACUC Challenges in Invertebrate Research }\end{array}$ & & & \\
\hline & $52(1)$ & $\begin{array}{l}\text { Animal Models of Aging: Something Old, Something New } \\
\text { - Introduction: Animal Models of Aging: Something Old, Something New } \\
\text { - Mice as a Mammalian Model for Research on the Genetics of Aging } \\
\text { - Heterogeneous Stocks and Selective Breeding in Aging Research } \\
\text { - The Collaborative Cross: A Recombinant Inbred Mouse Population for the Systems Genetic Era } \\
\text { - Mindspan: Lessons from Rat Models of Neurocognitive Aging } \\
\text { - Successful Aging and Sustained Good Health in the Naked Mole Rat: A Long-Lived Mammalian } \\
\text { Model for Biogerontology and Biomedical Research } \\
\text { - The Marmoset as a Model of Aging and Age-Related Diseases } \\
\text { - Calorie Restriction and Aging in Nonhuman Primates } \\
\text { - The Development of Small Primate Models for Aging Research } \\
\text { - Candidate Bird Species for Use in Aging Research } \\
\text { - Aging Research 2011: Exploring the Pet Dog Paradigm } \\
\text { IACUC Issues Related to Animal Models of Aging }\end{array}$ & & & \\
\hline
\end{tabular}




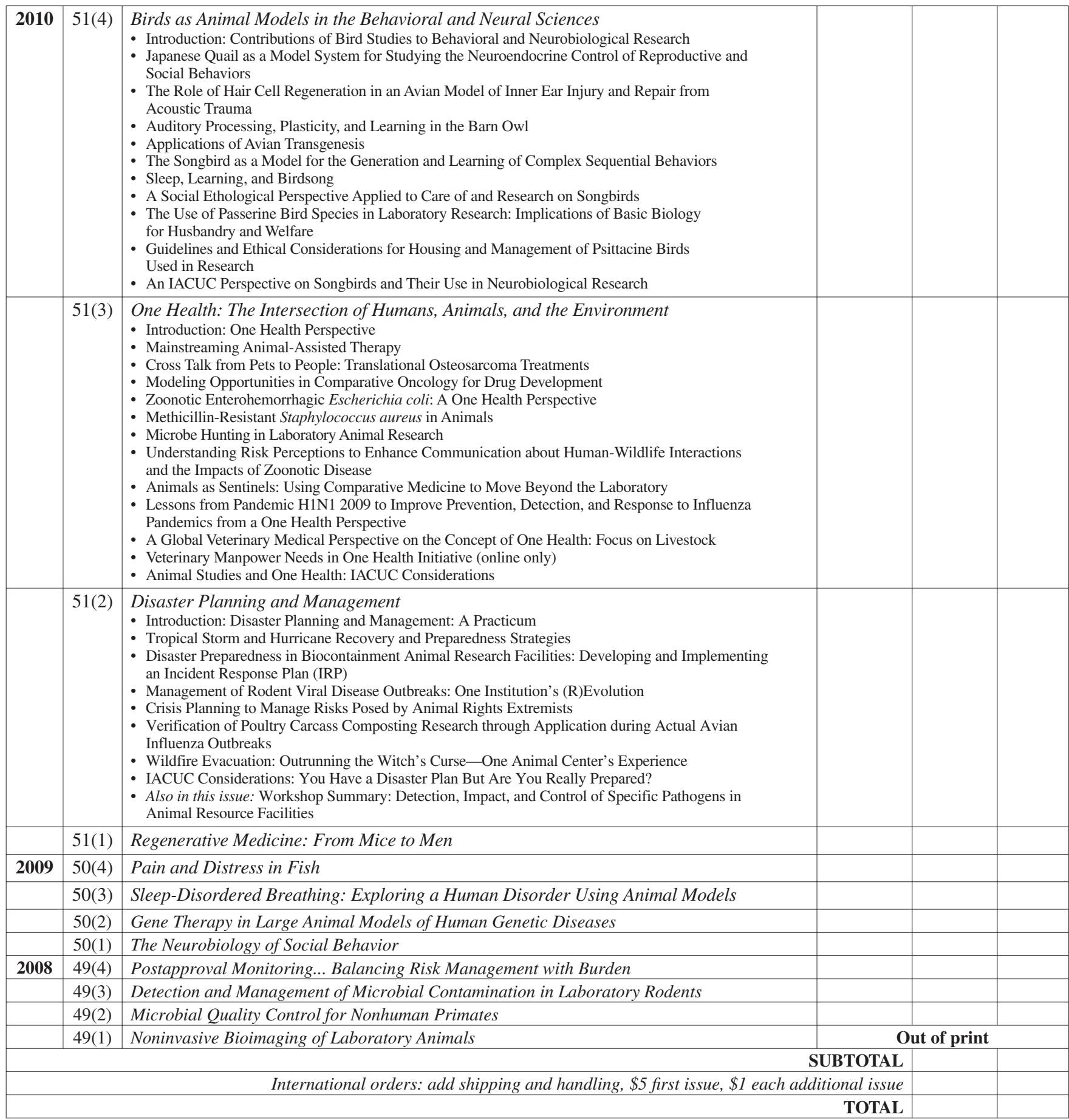

Please return this form with payment to:

\section{Institute for Laboratory Animal Research (ILAR)}

The National Academies

500 Fifth Street, NW

Washington, DC 20001

To learn more about our Associates program and how you can join, visit our website at www.nationalacademies.org/ilar.

I have enclosed a purchase order.

I have enclosed a check payable to ILAR for $\$$

(US dollars only).

Please charge my:

_ Visa ___ Master Card ___ American Express

Cardholder Name:

Card Number:

Exp.:

Please Print.

Name:

Address:

City:

Phone:

Email: 


\section{* Recently Released *}

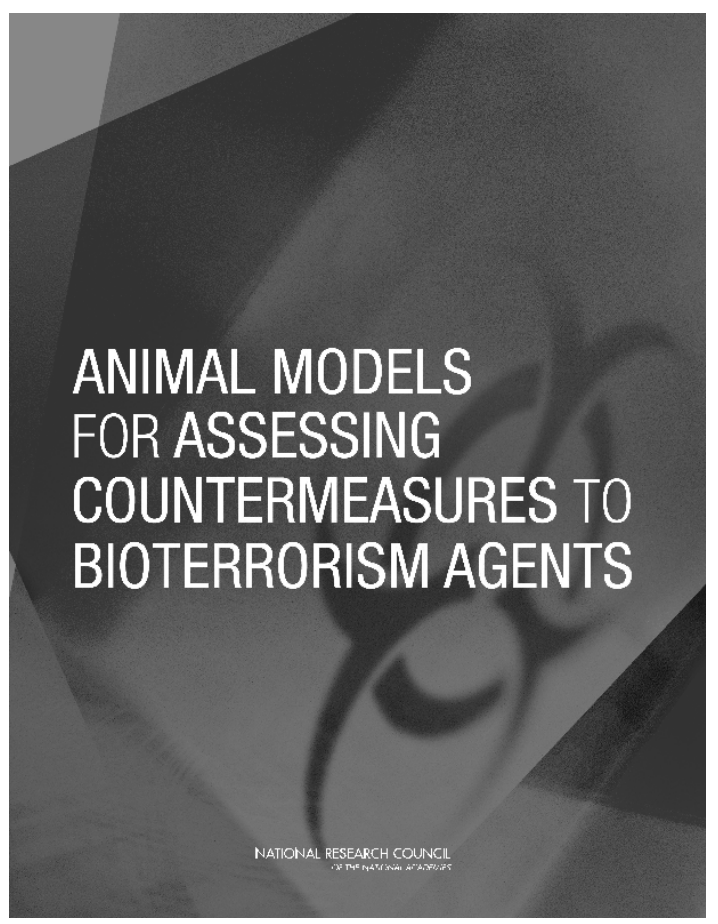

Transformational Medical Technologies, a component of the US Department of Defense, funds the development of medical countermeasures to protect warfighters against potential biothreats such as emerging infectious agents and toxins and future genetically engineered biological weapons. Because these pathogens are potentially lethal or cause debilitating diseases in humans, it is ethically impermissible to test the effectiveness of medical countermeasures on human volunteers. Instead, these products are tested on laboratory animals under a legal mechanism developed by the US Food and Drug Administration. However, relying solely on animal models for the development of countermeasures to biothreats is challenging. In many cases, qualified animal models that can reasonably predict the efficacy of new products are not available, and developing better animal models can involve years of effort without guaranteed success. In addition, there are difficulties in establishing good alternatives to using laboratory animals for medical countermeasure evaluation prior to human administration. This report evaluates existing and candidate animal models for testing medical countermeasures against biothreats, addresses the process and feasibility of developing new animal models, and evaluates alternatives to the use of animals based on the Three Rs-refinement, reduction, and replacement of the use of animals in research and testing.

To order your copy

contact National Academies Press:

online (www.nap.edu) or by telephone (888 624 8373) 


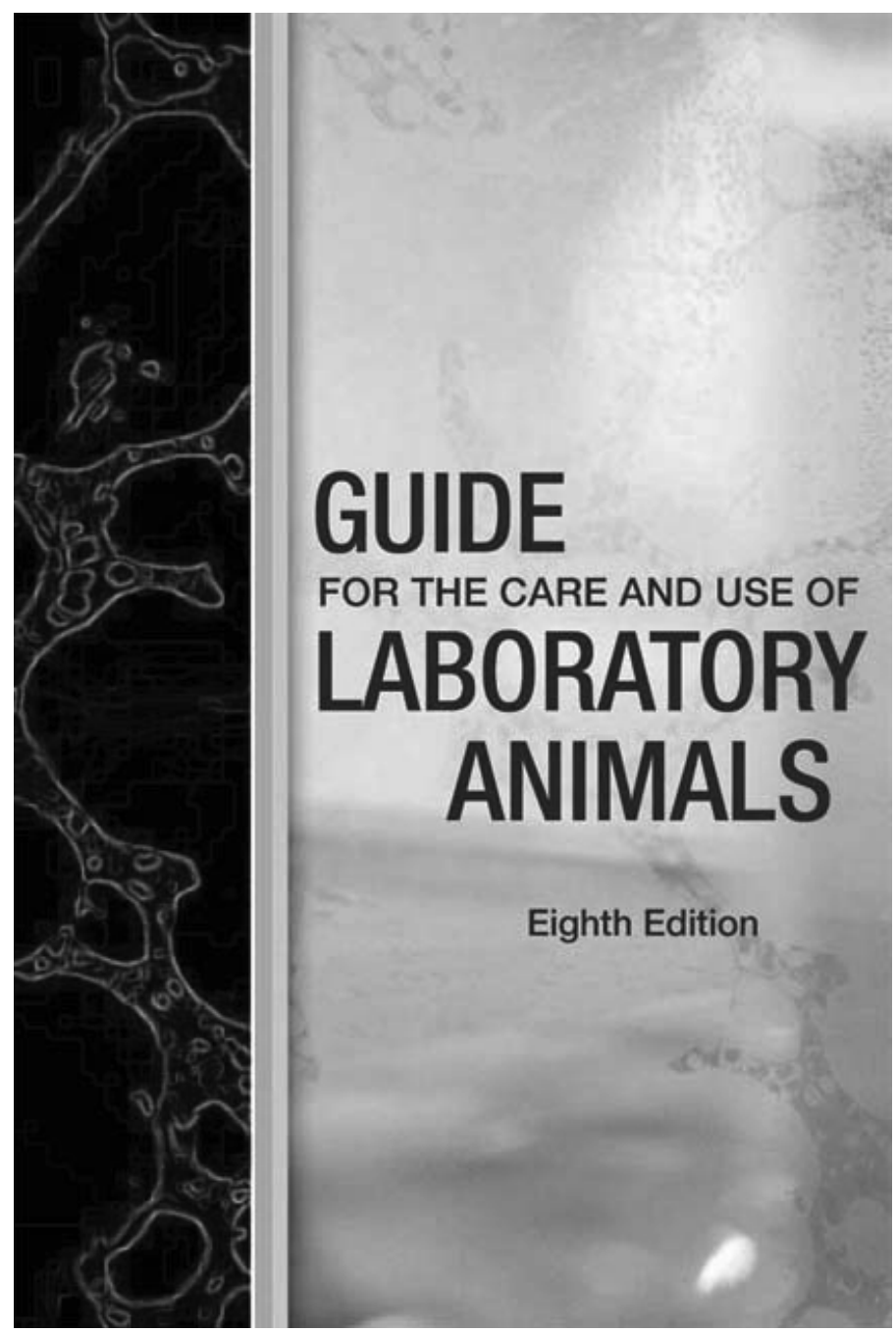

The Guide for the Care and Use of Laboratory Animals has been updated by a committee of experts to incorporate new scientific information on commonly used laboratory animals, including aquatic species, and provides extensive references. It is organized around major components of animal use: key concepts of animal care and use; animal care and use program (including the roles and responsibilities of the institutional official, attending veterinarian, and institutional animal care and use committee; regulatory considerations; program and personnel management; and program oversight); animal environment, husbandry, and management; veterinary care (including animal procurement and transportation, preventive medicine, and clinical care and management); and physical plant design considerations.

To order your copy:

Contact the National Academies Press at www.nap.edu or Call 888-624-8373 or 202-334-3313

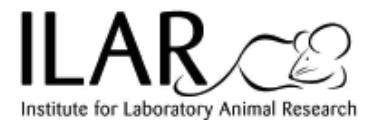





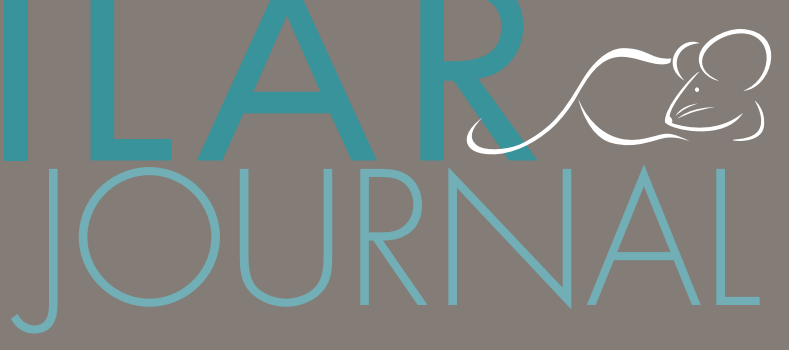

NATIONAL RESEARCH COUNCIL

OF THE NATIONAL ACADEMIES

\section{Address Correction Requested}

Remove from list

Change as shown

Detach corrected address label and mail

to ILAR at address inside front cover

\section{THE NATIONAL ACADEMIES}

Advisers to the Nation on Science, Engineering, and Medicine

The nation turns to the National Academies—National Academy of Sciences, National Academy of Engineering, Institute of Medicine, and National Research Councilfor independent, objective advice on issues that affect people's lives worldwide.

www.national-academies.org 